\title{
Scholarly Press
}

SMITHSONIAN CONTRIBUTIONS TO BOTANY $\bullet$ NUMBER I I 3
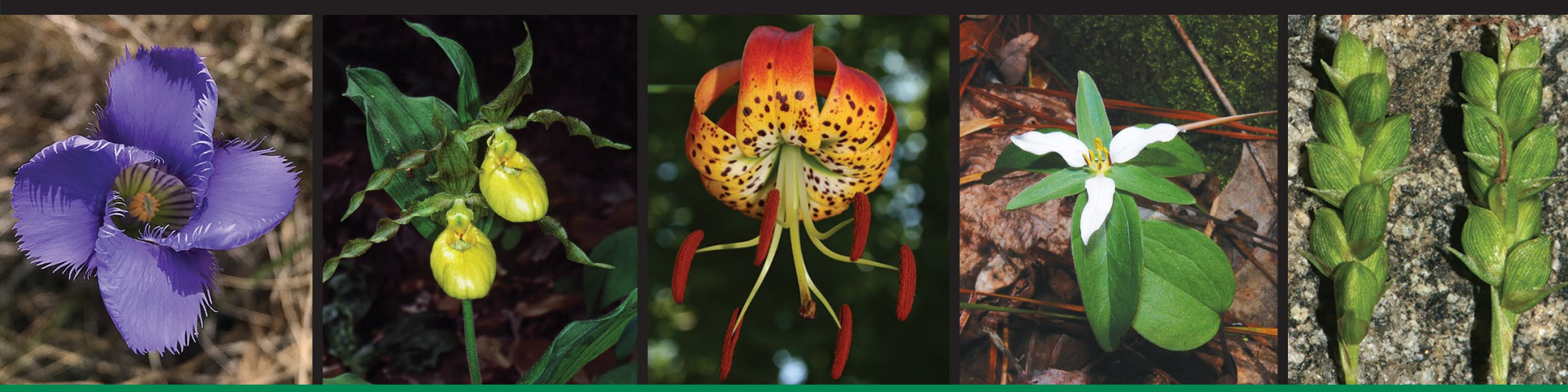

\section{Vascular Plants of Maryland, USA}

A Comprehensive Account of the State's Botanical Diversity

\author{
Wesley M. Knapp \\ and \\ Robert F. C. Naczi
}




\title{
SERIES PUBLICATIONS OF THE SMITHSONIAN INSTITUTION
}

Emphasis upon publication as a means of "diffusing knowledge" was expressed by the first Secretary of the Smithsonian. In his formal plan for the Institution, Joseph Henry outlined a program that included the following statement: "It is proposed to publish a series of reports, giving an account of the new discoveries in science, and of the changes made from year to year in all branches of knowledge." This theme of basic research has been adhered to through the years in thousands of titles issued in series publications under the Smithsonian imprint, commencing with Smithsonian Contributions to Knowledge in 1848 and continuing with the following active series:

\author{
Smithsonian Contributions to Anthropology \\ Smithsonian Contributions to Botany \\ Smithsonian Contributions to History and Technology \\ Smithsonian Contributions to the Marine Sciences \\ Smithsonian Contributions to Museum Conservation \\ Smithsonian Contributions to Paleobiology \\ Smithsonian Contributions to Zoology
}

In these series, the Smithsonian Institution Scholarly Press (SISP) publishes small papers and full-scale monographs that report on research and collections of the Institution's museums and research centers. The Smithsonian Contributions Series are distributed via exchange mailing lists to libraries, universities, and similar institutions throughout the world.

Manuscripts intended for publication in the Contributions Series undergo substantive peer review and evaluation by SISP's Editorial Board, as well as evaluation by SISP for compliance with manuscript preparation guidelines (available at https://scholarlypress.si.edu). SISP open access publications are licensed under Creative Commons licenses based on copyright status of content. Each is published initially online at https://smithsonian.figshare.com/ScholarlyPress and in print format in limited quantities. 


\title{
Vascular Plants of Maryland, USA
}

\section{A Comprehensive Account of the State's Botanical Diversity}

\author{
Wesley M. Knapp \\ and \\ Robert F. C. Naczi
}

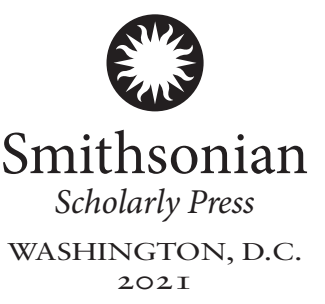




\section{ABSTRACT}

Knapp, Wesley M., and Robert F. C. Naczi. Vascular Plants of Maryland, USA: A Comprehensive Account of the State's Botanical Diversity. Smithsonian Contributions to Botany, number 113, viii + 151 pages, 1 figure, 2021. - This checklist provides the first complete, vouchered account of Maryland's vascular flora. In total, we discuss 3,525 taxa and document 2,918 established taxa for the state of Maryland, $71.8 \%$ of which are native and $28.2 \%$ of which are introduced. Of the native species, 737 (25.3\%) are tracked by the Maryland Natural Heritage Program as of conservation concern. We exclude 324 taxa reported from Maryland by previous authors and provide justifications for these exclusions.

Cover images, from left: Gentianopsis crinita (Gentianaceae), photo: W. Knapp; Cypripedium parviflorum var. parviflorum (Orchidaceae), photo: R. Wiegand; Lilium superbum (Liliaceae), photo: R. Naczi; Trillium pusillum var. virginianum (Melanthiaceae), photo: W. Knapp; Carex laxiculmis var. laxiculmis (Cyperaceae), photo: R. Naczi.

Published by SMITHSONIAN INSTITUTION SCHOLARLY PRESS

P.O. Box 37012, MRC 957

Washington, D.C. 20013-7012

https://scholarlypress.si.edu

Compilation copyright $@ 2021$ Smithsonian Institution

The rights to all text and images in this publication, including cover and interior designs, are owned either by the Smithsonian Institution, by contributing authors, or by third parties.

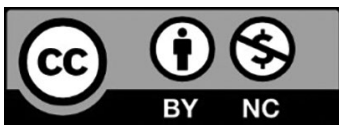

This work is licensed under a Creative Commons Attribution-NonCommercial 4.0 International (CC BY-NC 4.0) License.

Library of Congress Control Number: 2021931557

ISSN: 1938-2812 (online); 0081-024X (print)

Publication date (online): 17 May 2021

@ 0 The paper used in this publication meets the minimum requirements of the American National Standard for Permanence of Paper for Printed Library Materials Z39.48-1992. 


\section{Contents}

INTRODUCTION

CHECKLIST FORMAT

RESULTS 3

THE FUTURE OF MARYLAND FLORISTICS 4

DEFINITIONS OF NATURAL HERITAGE RANKS 4

State Ranking System $\quad \mathbf{4}$

Global Ranking System $\quad 4$

Qualifiers $\quad 4$

DEFINITIONS OF FEDERAL LISTED SPECIES STATUS 4

CHECKLIST OF FLORA

LYCOPHYTES $\mathbf{5}$

ISOETACEAE (Quillwort Family) $\quad \mathbf{5}$

LYCOPODIACEAE (Clubmoss Family) 5

SELAGINELLACEAE (Spikemoss Family) 5

FERNS $\quad \mathbf{5}$

ASPLENIACEAE (Spleenwort Family) $\quad 5$

ATHYRIACEAE (Lady Fern Family) 6

BLECHNACEAE (Chain Fern Family) 6

CYSTOPTERIDACEAE (Bladder Fern Family) 6

DENNSTAEDTIACEAE (Bracken Fern Family) 6

DIPLAZIOPSIDACEAE (Glade Fern Family) 6

DRYOPTERIDACEAE (Wood Fern Family) 6

EQUISETACEAE (Horsetail Family) 6

HYMENOPHYLLACEAE (Filmy Fern Family) $\quad 7$

LYGODIACEAE (Climbing Fern Family) $\quad 7$

MARSILEACEAE (Waterclover Family) $\quad 7$

ONOCLEACEAE (Sensitive Fern Family) 7

OPHIOGLOSSACEAE (Adder's-tongue Family) 7

OSMUNDACEAE (Royal Fern Family) $\quad 7$

POLYPODIACEAE (Polypody Family) $\quad 7$

PTERIDACEAE (Maidenhair Fern Family) $\quad 7$ 
NYCTAGINACEAE (Four-o'clock Family)

NYMPHAEACEAE (Water Lily Family) 61

NYSSACEAE (Tupelo Family)

OLEACEAE (Olive Family)

ONAGRACEAE (Evening-primrose Family) 62

ORCHIDACEAE (Orchid Family) 63

OROBANCHACEAE (Broomrape Family) 65

OXALIDACEAE (Woodsorrel Family) 65

PAPAVERACEAE (Poppy Family) 66

PASSIFLORACEAE (Passionflower Family)

PAULOWNIACEAE (Paulownia Family) 66

PENTHORACEAE (Ditch Stonecrop Family) 66

PHRYMACEAE (Lopseed Family) 66

PHYLLANTHACEAE (Leaf-flower Family)

PHYTOLACCACEAE (Pokeweed Family)

PLANTAGINACEAE (Plantain Family) 66

PLATANACEAE (Plane Tree Family) 68

PLUMBAGINACEAE (Leadwort Family)

POACEAE [Gramineae] (Grass Family)

PODOSTEMACEAE (Riverweed Family) 79

POLEMONIACEAE (Phlox Family) $\mathbf{8 0}$

POLYGALACEAE (Milkwort Family) $\quad \mathbf{8 0}$

POLYGONACEAE (Buckwheat Family)

PONTEDERIACEAE (Water Hyacinth Family) 82

PORTULACACEAE (Purslane Family) 82

POTAMOGETONACEAE (Pondweed Family)

PRIMULACEAE (Primrose Family) 82

RANUNCULACEAE (Buttercup/Crowfoot Family)

RESEDACEAE (Mignonette Family) $\mathbf{8 5}$

RHAMNACEAE (Buckthorn Family)

ROSACEAE (Rose Family) $\quad \mathbf{8 5}$

RUBIACEAE (Madder/Coffee Family)

RUPPIACEAE (Ditch Grass Family) $\quad 89$

RUTACEAE (Citrus/Rue Family)

SALICACEAE (Willow Family) 89

SANTALACEAE (Sandalwood Family) 90

SAPINDACEAE (Maple Family) 90

SAPOTACEAE (Sapodilla Family) 90

SARRACENIACEAE (Pitcher Plant Family) 90

SAURURACEAE (Lizard's-tail Family) $\quad 90$

SAXIFRAGACEAE (Saxifrage Family) 91

SCROPHULARIACEAE (Figwort Family) 91

SIMAROUBACEAE (Quassia Family) 91

SMILACACEAE (Greenbrier Family) 91

SOLANACEAE (Nightshade/Potato Family) 91

STAPHYLEACEAE (Bladdernut Family) 92

STYRACACEAE (Storax Family) 92

SYMPLOCACEAE (Sweetleaf Family) $9 \mathbf{9 2}$

TALINACEAE (Fameflower Family) 92

TAMARICACEAE (Tamarisk Family) 92

TETRACHONDRACEAE (Tetrachondra Family) 93

THYMELAEACEAE (Mezereum Family) 93

TOFIELDIACEAE (False Asphodel Family) 93

TYPHACEAE (Cattail Family) 
ULMACEAE (Elm Family)

URTICACEAE (Nettle Family)

93

VERBENACEAE (Verbena Family)

VIOLACEAE (Violet Family)

VITACEAE (Grape Family)

XYRIDACEAE (Yellow-eyed Grass Family)

ZOSTERACEAE (Eelgrass Family)

ZYGOPHYLLACEAE (Creosote-Bush Family)

EXCLUDED FLORA

LYCOPHYTES

ISOETACEAE (Quillwort Family)

FERNS

CYSTOPTERIDACEAE (Bladder Fern Family)

OPHIOGLOSSACEAE (Adder's-tongue Family)

PTERIDACEAE (Maidenhair Fern Family)

SALVINIACEAE (Floating Fern Family)

GYMNOSPERMS

PINACEAE (Pine Family)

FLOWERING PLANTS

ADOXACEAE (Moschatel Family)

ALISMATACEAE (Water-plantain Family)

AMARANTHACEAE (Amaranth Family)

AMARYLLIDACEAE (Amaryllis Family)

ANACARDIACEAE (Sumac/Cashew Family)

APIACEAE [Umbelliferae] (Carrot/Parsley Family)

APOCYNACEAE (Dogbane Family)

ARACEAE (Arum Family)

ASPARAGACEAE (Asparagus Family)

ASTERACEAE [Compositae] (Aster Family)

BERBERIDACEAE (Barberry Family)

BETULACEAE (Birch Family)

BORAGINACEAE (Borage Family)

BRASSICACEAE [Cruciferae] (Mustard Family)

BUXACEAE (Boxwood Family)

CAMPANULACEAE (Bellflower Family)

CANNABACEAE (Hemp Family)

CAPRIFOLIACEAE (Honeysuckle Family)

CARYOPHYLLACEAE (Pink Family)

COLCHICACEAE (Meadow Saffron Family)

COMMELINACEAE (Spiderwort Family)

CONVOLVULACEAE (Morning-glory Family)

CYPERACEAE (Sedge Family)

ELAEAGNACEAE (Oleaster Family)

ERICACEAE (Heath Family)

EUPHORBIACEAE (Spurge Family)

FABACEAE [Leguminosae] (Pea/Bean Family)

FAGACEAE (Beech Family)

GENTIANACEAE (Gentian Family)

GERANIACEAE (Geranium Family)

GROSSULARIACEAE (Gooseberry Family)

HYPERICACEAE (St. John's-wort Family)

IRIDACEAE (Iris Family) 
JUNCACEAE (Rush Family)

JUNCAGINACEAE (Arrowgrass Family)

LAMIACEAE [Labiatae] (Mint Family)

LILIACEAE (Lily Family)

LINACEAE (Flax Family)

LOGANIACEAE (Logania Family)

MALVACEAE (Mallow Family)

MELANTHIACEAE (Bunchflower Family)

MELASTOMATACEAE (Melastome Family)

MELIACEAE (Mahogany Family)

NELUMBONACEAE (Lotus Lily Family)

NYMPHAEACEAE (Water Lily Family)

OLEACEAE (Olive Family)

ONAGRACEAE (Evening-primrose Family)

ORCHIDACEAE (Orchid Family)

OROBANCHACEAE (Broomrape Family)

PAPAVERACEAE (Poppy Family)

PLANTAGINACEAE (Plantain Family)

POACEAE [Gramineae] (Grass Family)

POLEMONIACEAE (Phlox Family)

POLYGALACEAE (Milkwort Family)

POLYGONACEAE (Buckwheat Family)

POTAMOGETONACEAE (Pondweed Family) 


\title{
Vascular Plants of Maryland, USA
}

\section{A Comprehensive Account of the State's Botanical Diversity}

\author{
Wesley M. Knapp ${ }^{1,2 *}$ and Robert F. C. Naczi $i^{3,4}$
}

\section{INTRODUCTION}

${ }^{1}$ Maryland Natural Heritage Program, P.O. Box 68, Wye Mills, Maryland 21679, USA.

2 Present address: North Carolina Natural Heritage Program, Division of Land and Water Stewardship, North Carolina Department of Natural and Cultural Resources, 176 Riceville Road, Asheville, North Carolina 28805, USA.

${ }^{3}$ New York Botanical Garden, 2900 Southern Boulevard, Bronx, New York, 10458-5126, USA.

${ }^{4}$ Research Associate with Department of Botany, MRC-166, National Museum of Natural History, Smithsonian Institution, P.O. Box 37012, Washington, D.C. 20013-7012, USA.

* Correspondence: Wesley.Knapp@ncdcr.gov Manuscript received 8 March 2020; accepted 9 November 2020
This is the first peer-reviewed and vouchered checklist of the flora of Maryland. Our scope is the entire spontaneous vascular flora of the state. We intend for this document to be the definitive source for those interested in knowing which taxa occur within Maryland.

Maryland is the 42nd-largest state at $\sim 19,967 \mathrm{~km}^{2}\left(12,405 \mathrm{mi}^{2}\right)$ and is composed of 23 counties spanning five physiographic provinces (Figure 1). From east to west these are the Coastal Plain, Piedmont, Blue Ridge, Ridge and Valley, and Appalachian Plateaus physiographic provinces. The largest of these is the Coastal Plain Province, and the smallest is the Blue Ridge Province. Maryland contains a wide diversity of landscapes and geologic features for a state of its size. This diversity contributes to its botanical diversity as each physiographic province and its surficial geology support its own flora. Entire books have been devoted to the geology, landforms, and ecology of Maryland, and we recommend additional readings on these subjects: Maryland's Geology (Schmidt 1993), The Plant Life of Maryland (Shreve et al. 1910), and The Natural Communities of Maryland (Harrison 2016).

The first list of plants compiled for the state of Maryland was The Plant Life of Maryland (Shreve et al. 1910). These authors documented 1,400 species and varieties and stated "incomplete as it is, the list is sufficiently full to be worth publishing" (Shreve et al. 1910:383) and speculated that "the total flora when completely collected will probably approach 1,900 species" (Shreve et al 1910:385). The next checklist was A Catalog of the Vascular Plants of Maryland (Norton and Brown 1946). Norton and Brown (1946) gave the total flora as 2,416 species, forms, and varieties. The next and best-known treatments of the Maryland flora are Woody Plants of Maryland (Brown and Brown 1972) and Herbaceous Plants of Maryland (Brown and Brown 1984). In total, Brown and Brown's manuals included 2,632 taxa. Since this latest accounting, knowledge of the state's flora has undergone untold changes, and the need for a new checklist has become evident. The goals of the checklist are (1) to provide a complete, accurate, and updated accounting of the state's vascular plants validated by herbarium specimens and (2) to create a baseline of vetted specimen-vouchered information to assist understanding of floristic change as we move through the Anthropocene.

\section{CHECKLIST FORMAT}

The arrangement of major groups in the checklist is fern allies, ferns, gymnosperms, and flowering plants, with families organized alphabetically within each group. For each species, we provide scientific name, author(s), and common name. Authors of scientific 

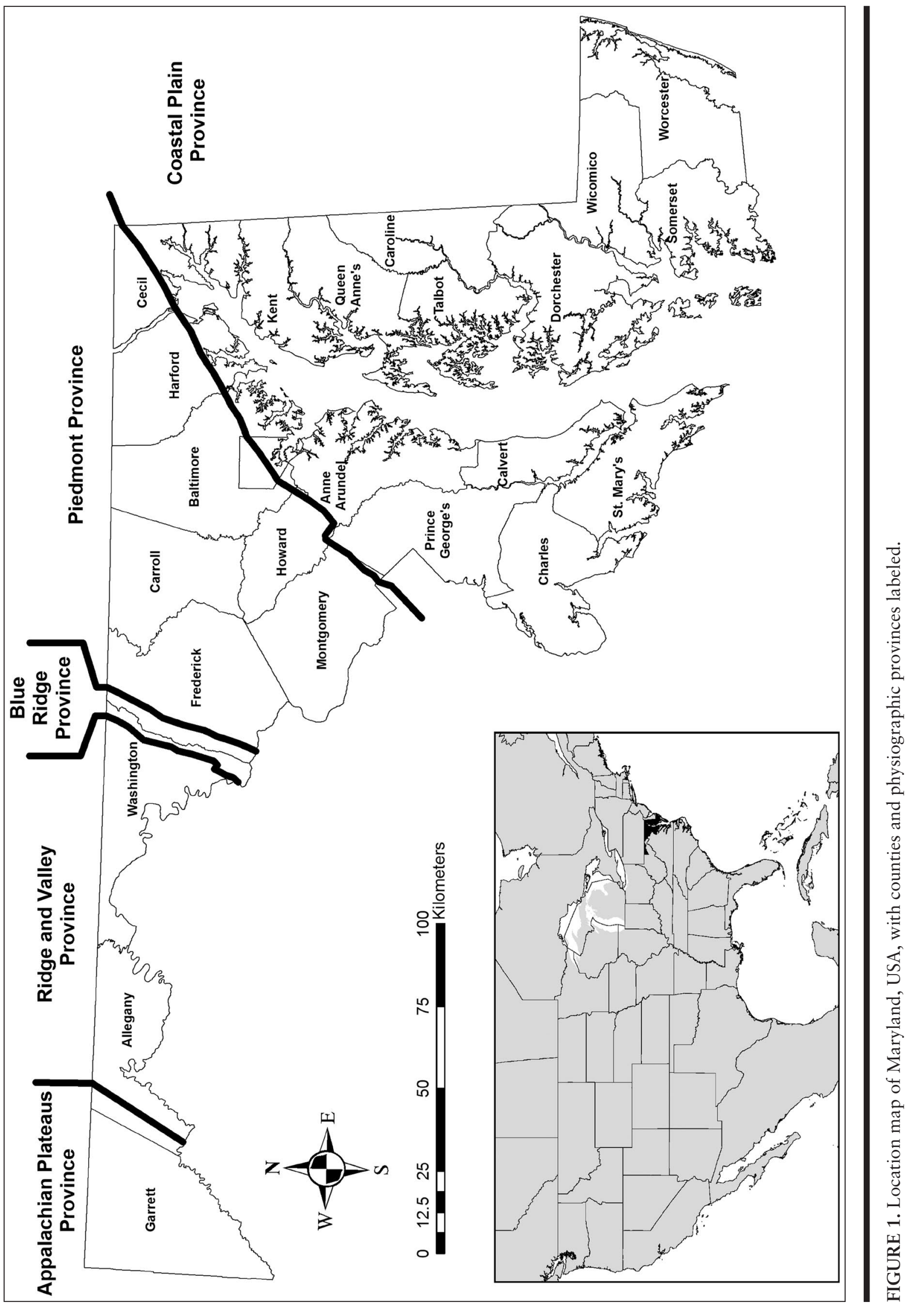
names follow Brummit and Powell (1992). We list a single vernacular name for each taxon as a common name. We used various sources for common names, including Weakley (2015), Maryland Plant Atlas Work Group (2016), Naczi et al. (2016), and the PLANTS Database from USDA (2018); ultimately, they were the choice of the senior author.

With very few exceptions, each taxon included in this checklist is vouchered by an herbarium specimen or, in a few instances, a photograph. Following each taxon, parentheses contain the collector, collector number, and abbreviation for herbarium of deposit. Herbarium abbreviations follow Index Herbariorum (Thiers 2020) with the exceptions of (1) the Assateague Island National Seashore Herbarium (Berlin, Maryland); (2) the herbarium of the Patuxent River Naval Air Station (Patuxent, Maryland); (3) the Cylburn Arboretum (Baltimore, Maryland); (4) the herbarium of Frostburg State University (Frostburg, Maryland); and (5) National Park Service Herbarium at Harpers Ferry (Harpers Ferry, West Virginia). Photographic vouchers are used sparingly and are accepted only when the species being documented is of significant rarity within the state (e.g., Cypripedium candidum), is readily identified with certainty from a photograph, or no other voucher is known. The depository of a photo voucher is indicated, similar to the herbarium of deposit. Photos indicated as being with MPA are hosted by the Maryland Plant Atlas (https://www.marylandplantatlas.org) and the photo voucher can be found on the corresponding online species page.

Taxa not native to Maryland are followed by a dagger $(\dagger)$. Exotic taxa that are invasive or noxious weeds are followed by a double dagger ( $\ddagger)$. We determined which taxa are invasive in consultation with various local experts, by our field observations, and by referencing various invasive plant species lists maintained by states adjacent to Maryland. Species of conservation concern tracked by the Maryland Natural Heritage Program (2018) are indicated by a white star in a black circle ( $)$, and the federal status designation is provided. Nomenclature is from a variety of sources, including the Flora of North America Editorial Committee (1993-), Weakley (2015), and Naczi (2016), but primarily follows the authors' judgements. We provide synonyms from sources published after Fernald (1950) indented and within square brackets below the accepted name (e.g., [Isoetes saccharata Engelm.]). Angiosperm family classification largely follows the Angiosperm Phylogeny Group (2016).

\section{Symbols Used to Characterize Species}

† Taxa not native to Maryland

$\ddagger$ Invasive or noxious weeds

Species of conservation concern

Taxa that appear to be waifs are clearly documented with "Waif" following the entry. The en-dash symbol ( - ) precedes any waif to indicate it is not an established component of the flora. We suspect that numerous species now considered waifs may already be established or are in the process of becoming established, but data on degree of establishment are lacking.
The dash ( - ) also indicates taxa that we exclude, and these taxa are followed by the notation "—-see Excluded Flora section." A separate section detailing the excluded flora follows the main checklist. For each excluded taxon, we state the explicit reason(s) for each exclusion. We expect some taxa currently excluded will eventually be added to the flora. It is likely that specimens validating some attributions for reports we exclude are currently in backlogs of specimens awaiting mounting in herbaria.

The collections of Clyde Reed pose a significant challenge for the effort to cite specimens for each member of the flora. Reed (1964) reported 550 flowering plants from the chrome and manganese ore piles in Maryland and Virginia, about " $80 \%$ of which were new to either Robinson and Fernald (1908) or Gleason (1952)." Specimens validating these attributions are housed in the Reed Herbarium at MO or, rarely, duplicated at US. Most of these records are treated as waifs in this treatment.

\section{Herbarium and Photo Voucher Repository Abbreviations}

$\begin{array}{ll}\text { BALT } & \text { Towson University } \\ \text { BM } & \text { British Museum } \\ \text { CM } & \text { Carnegie Museum of Natural History } \\ \text { GA } & \text { University of Georgia } \\ \text { GH } & \text { Gray Herbarium of Harvard University } \\ \text { DOV } & \text { Delaware State University } \\ \text { MARY } & \text { University of Maryland } \\ \text { MO } & \text { Missouri Botanical Garden } \\ \text { MPA } & \text { Maryland Plant Atlas } \\ \text { NA } & \text { U.S. National Arboretum } \\ \text { NCU } & \text { University of North Carolina at Chapel Hill } \\ \text { NY } & \text { New York Botanical Garden } \\ \text { OXF } & \text { University of Oxford } \\ \text { PH } & \text { Academy of Natural Sciences of Philadelphia } \\ \text { TAWES } & \text { Maryland Department of Natural Resources } \\ \text { TRT } & \text { Royal Ontario Museum } \\ \text { US } & \text { Smithsonian Institution } \\ \text { WAT } & \text { University of Waterloo } \\ \text { WVA } & \text { West Virginia University }\end{array}$

\section{RESULTS}

In total, 3,525 taxa are vetted in this checklist. The current established flora is known to consist of 2,918 taxa, composed of 2,094 native taxa $(71.8 \%)$ and 824 established non-native taxa $(28.2 \%)$. Of the native species, $737(25.3 \%)$ are tracked by the Maryland Natural Heritage Program. An additional 283 waifs are explicitly indicated and discussed. This checklist adds 323 established taxa to the number provided by the Brown and Brown manuals $(1972,1984)$. The number of additions to the flora since Brown and Brown $(1972,1984)$ is significantly more than 321 . This number is clearly determined only after the species that are being excluded in this checklist have been taken into consideration. There are 324 taxa formally excluded from 
the Maryland flora. Many of these were included in the total number of taxa treated in Brown and Brown's manuals. When these excluded taxa are removed, the true difference between this current checklist and the Brown and Brown flora is 638 taxa, a $21 \%$ increase. Of these 638 additions, 355 are native and 282 are non-native. Of the 355 native additions, $92(26 \%)$ are considered rare.

\section{THE FUTURE OF MARYLAND FLORISTICS}

Though the vascular plants of Maryland are relatively well known, we expect future field and herbarium workers will add taxa to the documented flora of the state. Especially useful will be investigations of species expanding their ranges, including non-native species becoming established in our region (Naczi 2016). To bolster our claim that the established number of taxa in Maryland will increase, we cite as an example the 31 taxa added to the known flora of Maryland since 2001, both natives and non-natives (Knapp et al. 2011; Longbottom et al. 2016).

\section{DEFINITIONS OF NATURAL HERITAGE RANKS}

The Maryland Natural Heritage Program uses a system in which each species is assigned two ranks, one representing its rangewide or global status ( $G$ rank) and one representing its status in the state ( $\mathrm{S}$ rank). Species with a rank of 1 are most critically imperiled; those with a rank of 5 are most secure.

\section{State Ranking System}

S1 Critically imperiled because of extreme rarity (5 or fewer occurrences of very few remaining individuals or extent) or because of some factor(s) making the species especially vulnerable to extirpation from Maryland.

S2 Imperiled in Maryland because of rarity (6-20 occurrences or few remaining individuals or acres) or because of some factor(s) making it very vulnerable to extirpation from Maryland.

S3 Rare or uncommon in Maryland (on the order of 21-100 occurrences).
S3.1 Vulnerable and globally rare. Actively tracked, and Maryland occurrences may be critical to the long-term security of the species.

S4 Apparently secure in Maryland, with many occurrences.

S5 Demonstrably secure in Maryland and quite frequent.

SH Of historical occurrence, not verified in the past 20 years.

SU Status uncertain.

SX Apparently extirpated from Maryland.

S? $\quad$ Not ranked to date.

\section{Global Ranking System}

G1 Critically imperiled globally (5 or fewer occurrences).

G2 Imperiled globally (6-20 occurrences).

G3 Either very rare and local throughout its range or found locally in a restricted range (21-100 occurrences).

G4 Apparently secure globally.

G5 Demonstrably secure globally.

GH Of historical occurrence throughout its range.

GU Possibly in peril range wide but status uncertain.

GX Believed to be extinct throughout range.

G? Not ranked to date.

G\#T\# Rank for subspecies or varieties; \# = 1, 2, 3, 4, 5, H, $\mathrm{U}, \mathrm{X}$, or ?.

\section{QUALIFIERS}

NR Not ranked.

Q Indicates the taxon has questionable taxonomic status.

$\mathrm{T}$ Indicates the infraspecific rank.

? A rank qualifier, which may span two to three ranks.

\section{DEFINITIONS OF FEDERAL LISTED SPECIES STATUS}

LE Endangered species: in danger of extinction throughout all or a significant portion of the range.

LT Threatened species: likely to become an endangered species in the foreseeable future throughout all or a significant portion of the range. 


\section{Checklist of Flora}

\section{LYCOPHYTES}

\section{ISOETACEAE}

(Quillwort Family)

Isoetes engelmannii A. Braun, Engelmann's Quillwort, S3/G4 (McAvoy 5594 DOV)

Isoetes riparia Engelm. ex A. Braun, Riverbank Quillwort, SU/G5? (McAvoy 1171 DOV)

[Isoetes saccharata Engelm.]

- Isoetes tuckermanii A. Braun, Tuckerman's Quillwort-see Excluded Flora section

\section{LYCOPODIACEAE}

(Clubmoss Family)

Dendrolycopodium dendroideum (Michx.) A. Haines, Tree Clubmoss (Cusick 25724 NCU)

[Lycopodium dendroideum Michx.; L. obscurum L. var. dendroideum (Michx.) Eaton]

Dendrolycopodium hickeyi (W. H. Wagner, Beitel, \& R. C. Moran) A. Haines, Hickey's Tree Clubmoss (Redman 6330 BALT)

[Lycopodium hickeyi W. H. Wagner, Beitel, \& R. C. Moran]

Dendrolycopodium obscurum (L.) A. Haines, Flat-branched Tree Clubmoss (Dillard s.n. DOV)

[Lycopodium obscurum L.]

Diphasiastrum digitatum (Dill. ex A. Braun) Holub, Southern Ground Cedar (McAvoy 3451 DOV)

[Lycopodium complanatum L. var. flabelliforme Fernald; L. digitatum Dill. ex A. Braun; L. flabelliforme (Fernald) Blanch.]

Diphasiastrum tristachyum (Pursh) Holub, Blue Ground Cedar, - S3/G5 (McAvoy 4454 DOV)

[Lycopodium tristachyum Pursh]

Huperzia lucidula (Michx.) Trevis., Shining Firmoss (Tatnall 4049 DOV)

[Lycopodium lucidulum Michx.]

Huperzia porophila (F. E. Lloyd \& Underw.) Holub, Rock Firmoss, SX/G4 (Boone s.n. TAWES)

[Lycopodium porophilum F. E. Lloyd \& Underw.]

Lycopodiella alopecuroides (L.) Cranfill, Foxtail Bog Clubmoss (Naczi 11548 DOV)

[Lycopodium alopecuroides L.]
Lycopodiella appressa (Chapm.) Cranfill, Southern Bog Clubmoss (Ebert \& Holt 294EH DOV)

[Lycopodium adpressum (Chapm.) F. E. Lloyd \& Underw.] Lycopodiella inundata (L.) Holub, Bog Clubmoss, S2/G5 (Thompson s.n. TAWES)

Lycopodium clavatum L., Running Pine (Knapp 988 DOV)

Pseudolycopodiella caroliniana (L.) Holub, Carolina Clubmoss, - S1/G5 (Steury 990911.7 US)

[Lycopodium carolinianum L.]

Spinulum annotinum (L.) A. Haines, Stiff Clubmoss (Knapp 989 DOV)

[Lycopodium annotinum L.]

\section{SELAGINELLACEAE}

(Spikemoss Family)

Selaginella apoda (L.) Fernald, Meadow Spikemoss (Otis s.n. DOV) [Lycopodioides apoda (L.) Kuntz]

Selaginella rupestris (L.) Spring, Ledge Spikemoss (Schultz s.n. DOV)

[Bryodesma rupestre (L.) Soják]

\section{FERNS}

\section{ASPLENIACEAE}

(Spleenwort Family)

Asplenium bradleyi D. C. Eaton, Bradley's Spleenwort, S1/G4 (Maxon 425 US)

Asplenium montanum Willd., Mountain Spleenwort (Lea 3286 DOV)

Asplenium pinnatifidum Nutt., Lobed Spleenwort, S1/G4 (Riefner 80396 US)

Asplenium platyneuron (L.) Britton, Sterns, \& Poggenb., Ebony Spleenwort (Knapp 2836 DOV)

[Asplenium platyneuron var. bacculum-rubrum (Featherm.) Fernald]

Asplenium resiliens Kunze, Blackstem Spleenwort, S1/G5 (Riefner 81-1 MARY)

[Asplenium parvulum M. Martens \& Galeotti]

Asplenium rhizophyllum L., Walking Fern Spleenwort (Chapin s.n. US)

[Camptosorus rhizophyllus (L.) Link] 
Asplenium ruta-muraria L., Wallrue Spleenwort, S3/G5 (Riefner 81-78 MARY)

[Asplenium cryptolepis Fernald; A. ruta-muraria var. cryptolepis (Fernald) Wherry]

Asplenium scolopendrium L. var. americanum (Fernald) Kartesz \& Gandhi, American Hart's Tongue Fern, † (Knight 10 MARY)

[Phyllitis scolopendrium (L.) Newman var. americana Fernald]

Asplenium trichomanes L. ssp. trichomanes, Maidenhair Spleenwort (Knapp \& Frye 1385 DOV)

\section{ATHYRIACEAE}

(Lady Fern Family)

Athyrium angustum (Willd.) C. Presl, Lady Fern (McAvoy 6023 DOV)

[Athyrium filix-femina (L.) Roth ssp. angustum (Willd.) R. T. Clausen; A. filix-femina var. angustum (Willd.) G. Lawson; A. filix-femina var. michauxii (Spreng.) Farw.]

Athyrium asplenioides (Michx.) Desv., Southern Lady Fern (McAvoy 6059 DOV)

[Asplenium filix-femina (L.) Bernh. ssp. asplenioides (Desv.) Hultén; A. filix-femina var. asplenioides Farw.]

Deparia acrostichoides (Sw.) M. Kato, Silver False Spleenwort (Earle $2489 \mathrm{PH})$

[Asplenium acrostichoides Sw.; Athyrium thelypterioides (Michx.) Desv.; Diplazium acrostichoides (Sw.) Butters]

\section{BLECHNACEAE}

(Chain Fern Family)

Anchistea virginica (L.) K. Presl, Virginia Chain Fern (Tatnall 4610 DOV)

[Woodwardia virginica (L.) Sm.]

Lorinseria areolata (L.) K. Presl, Netted Chain Fern (Tatnall 4566 DOV)

[Woodwardia areolata (L.) T. Moore]

\section{CYSTOPTERIDACEAE}

(Bladder Fern Family)

Cystopteris bulbifera (L.) Bernh., Bulblet Fern, S3/G5 (Riefner 81-47 MARY)

Cystopteris protrusa (Weath.) Blasdell, Lowland Brittle Fern (Steury 990521 US)

[Cystopteris fragilis (L.) Bernh. var. protrusa Weath.]

Cystopteris tennesseensis Shaver, Tennessee Bladder Fern, S1/G5 (Redman 4494 US)

Cystopteris tenuis (Michx.) Desv., Upland Brittle Bladder Fern (Wagner 2001 US)

- Gymnocarpium appalachianum K. M. Pryer \& Haufler, Appalachian Oak Fern-see Excluded Flora section.

Gymnocarpium dryopteris (L.) Newman, Northern Oak Fern, - S1/G5 (Thompson s.n. TAWES)
DENNSTAEDTIACEAE

(Bracken Fern Family)

Dennstaedtia punctilobula (Michx.) T. Moore, Hayscented Fern (Tatnall 4565 DOV)

Pteridium aquilinum (L.) Kuhn var. latiusculum (Desv.) L. Underw. ex A. Heller, Eastern Bracken Fern (McAvoy 2133 DOV)

[Pteridium aquilinum ssp. latiusculum (Desv.) R. T. Clausen; Pteris aquilina L.; P. latiuscula Desv.]

Pteridium aquilinum (L.) Kuhn var. pseudocaudatum (Clute) A. Heller, Southern Bracken Fern (McAvoy 2742 DOV) [Pteridium aquilinum ssp. pseudocaudatum (Clute) Hultén; Pteris aquilina L. var. pseudocaudata Clute]

\section{DIPLAZIOPSIDACEAE}

(Glade Fern Family)

Homalosorus pycnocarpos (Spreng.) Pic. Serm., Glade Fern, - S2/G5 (Knapp 2340 TAWES)

[Asplenium angustifolium Michx.; Athyrium pycnocarpon (Spreng.) Tidestr.; Diplazium pycnocarpon (Spreng.) M. Broun]

DRYOPTERIDACEAE

(Wood Fern Family)

- Cyrtomium fortunei J. Sm., Asian Netvein Hollyfern, † Waif (Renzi Photo Voucher MPA)

Dryopteris campyloptera Clarkson, Mountain Wood Fern, - S1/G5 (Knapp 991 TAWES)

Dryopteris carthusiana (Vill.) H. P. Fuchs, Spinulose Shield Fern (Knapp 526 DOV)

[Dryopteris spinulosa (O. F. Müll.) Watt]

Dryopteris celsa (W. Palmer) Knowlt., T. S. Palmer \& Pollard ex Small, Log Fern, S3/G4 (Hirst 1078 DOV)

[Dryopteris atropalustris Small]

Dryopteris clintoniana (D. C. Eaton) Dowell, Clinton's Wood Fern, S1/G5 (Knapp 506a TAWES)

Dryopteris cristata (L.) A. Gray, Crested Shield Fern (Hirst \& Wilson 1172 DOV)

Dryopteris goldiana (Hook. ex Goldie) A. Gray, Goldie's Wood Fern, S2/G4G5 (Tatnall 205 DOV)

Dryopteris intermedia (Willd.) A. Gray, Evergreen Wood Fern (Tatnall 4564 DOV)

Dryopteris marginalis (L.) A. Gray, Marginal Wood Fern (Tatnall 4739 DOV)

Polystichum acrostichoides (Michx.) Schott, Christmas Fern (Longbottom 10803 DOV)

EQUISETACEAE

(Horsetail Family)

Equisetum arvense L., Field Horsetail (Knapp 1351 DOV)

Equisetum fluviatile L., Water Horsetail, S1/G5 (McAvoy 2466 DOV) 
Equisetum byemale L. ssp. affine (Engelm.) Calder \& Roy L. Taylor, Scouringrush (Hirst 691 DOV)

[Equisetum byemale var. affine (Engelm.) A. A. Eaton; E. praealtum Raf.; E. robustum A. Braun]

Equisetum sylvaticum L., Woodland Horsetail, S1/G5 (Fessenden 5254 US)

[Equisetum sylvaticum var. multiramosum Wherry]

\section{HYMENOPHYLLACEAE (Filmy Fern Family)}

Crepidomanes intricatum (Farrar) Ebihara \& Weakley, Weft Fern (Farrar 81-9-23-1 US)

[Trichomanes intricatum Farrar]

\section{LYGODIACEAE (Climbing Fern Family)}

Lygodium palmatum (Bernh.) Sw., Climbing Fern, S2/G4 (McAvoy 3256 DOV)

\section{MARSILEACEAE}

(Waterclover Family)

Marsilea quadrifolia L., European Water Fern, † (Fessenden 5592 US)

Pilularia americana A. Braun, American Pillwort, † (Metheny 0002 MARY)

\section{ONOCLEACEAE}

(Sensitive Fern Family)

Matteuccia struthiopteris (L.) Tod., Ostrich Fern, S2S3/G5 (Waters s.n. US)

[Matteuccia struthiopteris var. pensylvanica (Willd.) C. V. Morton]

Onoclea sensibilis L., Sensitive Fern (McAvoy 3668 DOV)

\section{OPHIOGLOSSACEAE (Adder's-tongue Family)}

Botrychium angustisegmentum (Pease \& A. H. Moore) Fernald, Lanceleaf Grape Fern, S1/G5 (Redman 9124 BALT)

[Botrychium lanceolatum (S. G. Gmel.) Ångst. ssp. angustisegmentum (Pease \& A. H. Moore) R. T. Clausen]

Botrychium matricariifolium (Retz.) A. Braun ex W. D. J. Koch, Chamomile Grape Fern, S1?/G5 (Maxon 11049 US)

Botrychium simplex E. Hitchc., Least Grape Fern, SH/G5 (Coe s.n. US)

Botrypus virginianus (L.) Michx., Rattlesnake Fern (Hirst \& Wilson 1230 DOV)

[Botrychium virginianum (L.) Sw.; Osmundopteris virginiana (L.) Small]

Ophioglossum pusillum Raf., Northern Adder's-tongue (Phillips 016 DOV)

Ophioglossum vulgatum L., Southern Adder's-tongue (Hirst 854 DOV)
[Ophioglossum pycnostichum (Fernald) Á. Löve \& D. Löve; O. vulgatum var. pycnostichum Fernald]

Sceptridium biternatum (Savigny) Lyon, Sparse-lobe Grape Fern (Knapp 2286 DOV)

Sceptridium dissectum (Spreng.) Lyon, Cutleaf Grape Fern (Knapp 2285 DOV)

[Botrychium dissectum Spreng. var. obliquum (Muhl. ex Willd.) Clute; B. obliquum Muhl. ex Willd.]

- Sceptridium jenmanii (Underw.) Lyon, Alabama Grape Fernsee Excluded Flora section.

[Botrychium jenmanii Underw.]

Sceptridium multifidum (S. G. Gmel.) Tagawa, Leathery Grape Fern, SH/G5 Extirpated-no specimen known to exist but reported by firsthand observers, Reed (1943) and Redman (1991). This species is now extirpated.

[Botrychium multitifidum (S. G. Gmel.) Rupr.]

Sceptridium oneidense (Gilbert) Holub, Blunt-lobe Grape Fern, - S1/G4 (Reed $3862 \mathrm{MO})$

\section{OSMUNDACEAE}

(Royal Fern Family)

Claytosmunda claytoniana (L.) Metzgar \& Rouhan, Interrupted Fern (Longbottom et al. 9000 DOV)

[Osmunda claytoniana L.]

Osmunda regalis L. var. spectabilis (Willd.) A. Gray, Royal Fern (Hirst s.n. DOV)

Osmundastrum cinnamomeum (L.) C. Presl var. cinnamomeum, Cinnamon Fern (Knapp 2800 NY)

[Osmunda cinnamomea L. var. cinnamomea]

Osmundastrum cinnamomeum (L.) C. Presl var. glandulosum (Waters) McAvoy, Glandular Cinnamon Fern (Knapp 2801 NY)

[Osmunda cinnamomea L. var. glanduosa Waters]

\section{POLYPODIACEAE}

(Polypody Family)

Pleopeltis michauxiana (Weath.) Hickey \& Sprunt, Resurrection Fern, S3/G5 (McAvoy 4261 DOV)

[Marginaria polypodioides (L.) Tidestr.; Pleopeltis polypodioides (L.) E. B. Andrews \& Windham ssp. michauxiana (Weath.) E. B. Andrews \& Windham; Polypodium polypodioides (L.) Watt]

Polypodium appalachianum Haufler \& Windham, Appalachian Rockcap Fern (Perdew 1237 MARY)

Polypodium virginianum L., Rock Polypody (Lea s.n. DOV) [Polypodium vulgare L.]

PTERIDACEAE

(Maidenhair Fern Family)

- Adiantum aleuticum (Rupr.) C. A. Paris, Aleutian Maidenhair Fern-see Excluded Flora section. 
- Adiantum capillus-veneris L., Southern Maidenhair Fern, $\dagger$ Waif reported by Benedict (1924) from Montgomery County, locality later destroyed (McAtee 1930)

Adiantum pedatum L., Northern Maidenhair Fern (Naczi \& Pennell 719 DOV)

Myriopteris lanosa (Michx.) Grusz \& Windman, Hairy Lip Fern (Maxon 6327 US) [Cheilanthes lanosa (Michx.) D. C. Eaton]

Pellaea atropurpurea (L.) Link, Purple-stem Cliffbrake (McAvoy 5936 DOV)

Pellaea glabella Mett. ex Kuhn ssp. glabella, Smooth Cliffbrake, - S1/G5T5 (Riefner 80-539 MARY)

- Pteris cretica L., Cretan Fern, † Waif Reported by Koerber (1962)

[Pycnodoria cretica (L.) Small]

- Pteris multifida Poir., Spider Brake, † Waif (Norton 1249 MARY)

Vittaria appalachiana Farrar \& Mickel, Appalachian Vittaria (Cusick 23891A MARY)

\section{SALVINIACEAE}

(Floating Fern Family)

Azolla caroliniana Willd., Eastern Mosquito Fern, †? (Hirst 588 DOV)

- Salvinia minima Baker, Water Spangles—see Excluded Flora section.

[Salvinia rotundifolia auct. non Willd.]

\section{THELYPTERIDACEAE}

(Marsh Fern Family)

Parathelypteris noveboracensis (L.) Ching, New York Fern (McAvoy 3703 DOV)

[Dryopteris noveboracensis (L.) A. Gray; Thelypteris noveboracensis (L.) Nieuwl.]

Phegopteris connectilis (Michx.) Watt, Northern Beech Fern, - S2/G5 (Boone s.n. TAWES)

[Thelypteris phegopteris Sloss.]

Phegopteris hexagonoptera (Michx.) Fée, Broad Beech Fern (Tatnall 4481 DOV)

[Thelypteris hexagonoptera (Michx.) Weath.]

Thelypteris palustris Schott var. pubescens (G. Lawson) Fernald, Eastern Marsh Fern (McAvoy 2675 DOV)

[Dryopteris thelypteris (L.) A. Gray; Thelypteris palustris var. haleana Fernald; T. thelypteris Nieuwl.]

Thelypteris simulata (Davenp.) Nieuwl., Bog Fern, S2/G4G5 (Sipple 2203 DOV)
WOODSIACEAE

(Cliff Fern Family)

Woodsia ilvensis (L.) R. Br., Rusty Woodsia, S2/G5 (Boone s.n. TAWES)

Woodsia obtusa (Spreng.) Torr. ssp. obtusa, Blunt-lobed Cliff Fern (Steury 990715.2 US)

\section{GYMNOSPERMS}

CUPRESSACEAE

(Cypress Family)

Chamaecyparis thyoides (L.) Britton, Sterns, \& Poggenb., Atlantic White Cedar, S3/G4 (McAvoy 4621 DOV)

Juniperus communis L. var. depressa Pursh, Dwarf Juniper, SH/G5 (Reed 32579 TAWES)

Juniperus virginiana L. var. virginiana, Eastern Red Cedar (Longbottom 9917 DOV)

[Sabina virginiana (L.) Antoine]

Taxodium distichum (L.) Rich., Bald Cypress (Hirst \& Wilson 713 DOV)

Thuja occidentalis L., Northern White Cedar, S1/G5 (Hill 10709 MARY)

PINACEAE

(Pine Family)

Abies balsamea (L.) Mill., Balsam Fir, S1/G5 (Thompson s.n. TAWES)

Larix decidua Mill., European Larch, † Waif (Kekich 40 MARY) Larix laricina (Du Roi) K. Koch, American Larch, S1/G5/ † (Lewis s.n. BALT) - rare and naturally occurring but also planted and rarely escaping

- Picea abies (L.) H. Karst, Norway Spruce-see Excluded Flora section.

- Picea glauca (Moench) Voss, White Spruce-see Excluded Flora section. [P. glauca var. densata Bailey]

- Picea pungens Engelm., Blue Spruce-see Excluded Flora section.

Picea rubens Sarg., Red Spruce, S3/G5 (Knapp \& Feller 2494 MARY)

Pinus echinata Mill., Shortleaf Pine (Knapp 1424 DOV)

Pinus nigra Arnold, Austrian Pine, † (Hill 16024 Assateague Island National Seashore Herbarium) - possibly merely persisting after cultivation

Pinus palustris Mill., Longleaf Pine, † (Knapp s.n. TAWES)

Pinus pungens Lamb., Table Mountain Pine (Tepfer 1390 MARY) 
Pinus resinosa Aiton, Red Pine, $\dagger$ (Dubik 2 MARY)

Pinus rigida Mill., Pitch Pine (McAvoy 3317 DOV)

Pinus serotina Michx., Pond Pine (McAvoy 6103 DOV)

Pinus strobus L., Eastern White Pine (Roller 1038 US)

Pinus sylvestris L. var. sylvestris, Scotch Pine, † (Baltars 1911

US) - possibly merely persisting after cultivation

Pinus taeda L., Loblolly Pine (Redmond s.n. DOV)

Pinus thunbergiana Franco, Japanese Black Pine, † (Knapp 5270 MARY)

Pinus virginiana Mill., Virginia Pine (Tatnall 2433 DOV)

Tsuga canadensis (L.) Carrière, Eastern Hemlock (McAvoy 5800 DOV)

\section{TAXACEAE}

(Yew Family)

Taxus canadensis Marshall, Canadian Yew, S2/G5 (Reveal et al. 5321 MARY)

\section{FLOWERING PLANTS}

\section{ACANTHACEAE}

(Acanthus Family)

Justicia americana (L.) Vahl, Common Water-willow (Tatnall 1477 DOV)

[Dianthera americana L.]

Ruellia caroliniensis (J. F. Gmel.) Steud., Carolina Wild Petunia (Tatnall 386 DOV)

[Ruellia ciliosa Pursh var. bybrida (Pursh) A. Gray; R. parviflora (Nees) Britton]

Ruellia bumilis Nutt., Hairy Wild Petunia, S1/G5 (Wiegand Photo Voucher MPA)

[Ruellia ciliosa Pursh var. humilis (Nutt.) Britton]

Ruellia purshiana Fernald, Pursh's Wild Petunia, S1/G3 (Wiegand Photo Voucher MPA)

[Ruellia pedunculata Torr. ex A. Gray]

Ruellia strepens L., Limestone Wild Petunia, S2S3/G4G5 (Sipple 2114 DOV)

\section{ACORACEAE}

(Sweet Flag Family)

Acorus calamus L., European Calamus, † (Naczi 8040 DOV) [Acorus americanus (Raf.) Raf.]

\section{ADOXACEAE}

(Moschatel Family)

Sambucus canadensis L., Common Elderberry (Engh s.n. MARY)

[Sambucus nigra L. ssp. canadensis (L.) R. Bolli; S. simpsonii Rehder]

Sambucus racemosa L. var. pubens (Michx.) Koehne, Red Elderberry (Reveal \& Reveal 4990 MARY)

[Sambucus pubens Michx.]
Viburnum acerifolium L., Mapleleaf Viburnum (Reveal et al. 5394 MARY)

[Viburnum densiflorum Chapm.]

Viburnum cassinoides L., Northern Wild Raisin (Ditman s.n. MARY)

Viburnum dentatum L. var. dentatum, Southern Arrowwood (Norton s.n. MARY)

[Viburnum semitomentosum (Michx.) Rehder]

Viburnum dentatum L. var. lucidum Aiton, Northern Arrowwood (Turpin 349 MARY)

[Viburnum recognitum Fernald]

Viburnum dilatatum Thunb., Linden Viburnum, † (Knapp \& Kyde 4159 TAWES)

Viburnum lantana L., Wayfaring Tree, † (Gregory 6 MARY)

Viburnum lantanoides Michx., Alderleaf Viburnum, † (Schorck s.n. MARY)

[Viburnum alnifolium Marshall]

Viburnum lentago L., Nannyberry, S1/G5 (Brown s.n. Frostburg State University Herbarium)

- Viburnum molle Michx., Soft Arrowwood-see Excluded Flora section.

Viburnum nudum L., Possum-haw Viburnum (Ditman s.n. MARY)

Viburnum opulus L. var. opulus, Guelder Rose Viburnum, † (Salzman 24 MARY)

Viburnum prunifolium L., Smooth Black Haw (Naczi 11334 DOV)

Viburnum rafinesquianum Schult., Downy Arrowwood (Knapp 2717 DOV)

- Viburnum rufidulum Raf., Southern Black Haw—see Excluded Flora section.

Viburnum setigerum Hance, Tea Viburnum, $†$ (Ellis Photo Voucher MPA)

Viburnum sieboldii Miq., Siebold's Viburnum, † (Hill 11135 MARY)

AIZOACEAE

(Fig Marigold/Ice Plant Family)

Sesuvium maritimum (Walter) Britton, Sterns, \& Poggenb., Slender Sea Purslane, S1/G5 (Frye 5117 TAWES)

ALISMATACEAE (Water-plantain Family)

Alisma subcordatum Raf., Southern Water Plantain (McAvoy 2558 DOV)

[Alisma plantago-aquatica L. ssp. subcordatum (Raf.) Hultén]

- Alisma triviale Pursh, Northern Water Plantain-see Excluded Flora section.

Echinodorus cordifolius (L.) Griseb. ssp. cordifolius, Creeping Burhead, S1/G5 (McAvoy 1427 DOV)

[Echinodorus radicans (Nutt.) Engelm.] 
- Helanthium tenellum (Mart. ex Schult. \& Schult. f.) Britton, Little Burhead-see Excluded Flora section.

[Echinodorus tenellus (Mart. ex Schult. \& Schult. f.) Bucheanu]

Sagittaria australis (J. G. Sm.) Small, Appalachian Arrowhead (Steury 980817.6 US)

[Sagittaria longirostra (Micheli) J. G. Sm.; S. longirostra var. australis J. G. Sm.]

- Sagittaria brevirostra Mack. \& Bush, Midwestern Arrowhead-see Excluded Flora section.

Sagittaria calycina Engelm., Hooded Arrowhead (McAvoy 1163 DOV)

[Sagittaria montevidensis Cham. \& Schltdl. ssp. calycina (Engelm.) Bogin]

Sagittaria engelmanniana J. G. Sm., Engelmann's Arrowhead, S2/G5? (Naczi 8949 DOV)

- Sagittaria filiformis J. G. Sm., Threadleaf Arrowhead-see Excluded Flora section.

[Sagittaria stagnorum Small; S. subulata (L.) Buchenau var. gracillima J. G. Sm.]

Sagittaria graminea Michx., Grassleaf Arrowhead, SU/G5 (Leonard \& Leonard 6242 US)

[Sagittaria cycloptera C. Mohr]

Sagittaria lancifolia L. ssp. media (Micheli) Bogin, Bull-tongue Arrowhead (Gladu s.n. MARY)

[Sagittaria falcata Pursh; S. lancifolia var. falcata (Pursh) J. G. Sm.]

Sagittaria latifolia Willd. var. latifolia, Broadleaf Arrowhead (Norton s.n. MARY)

[Sagittaria obtusa Muhl. ex Willd.; S. viscosa C. Mohr]

Sagittaria latifolia Willd. var. pubescens (Muhl. ex Nutt.) J. G. Sm., Hairy Broadleaf Arrowhead (Norton s.n. MARY)

[Sagittaria pubescens Muhl. ex Nutt.]

Sagittaria rigida Pursh, Sessile-fruited Arrowhead, S1/G5 (Killip 13496 US)

Sagittaria spathulata (J. G. Sm.) Buchenau, Tidal Arrowhead, S2/G5 (Shull 304 US)

[Lophotocarpus spongiosus (Engelm.) J. G. Sm.; Sagittaria calycina Engelm. var. spongiosa Engelm.; S. montevidensis Cham. \& Schltdl. ssp. spongiosa (Engelm.) Bogin]

Sagittaria subulata (L.) Buchenau, Dwarf Arrowhead, SU/G4 (Strong et al. 1620 US)

- Sagittaria teres S. Watson, Slender Arrowhead-see Excluded Flora section.

\section{ALTINGIACEAE}

(Sweetgum Family)

Liquidambar styraciflua L., Sweetgum (Hill 9958 MARY)

\section{AMARANTHACEAE}

(Amaranth Family)

- Achyranthes aspera L. var. pubescens (Moq.) C. C. Towns., Rough Chaff-flower, † Waif (Reed $41157 \mathrm{MO}$ )

[Achyranthes aspera var. obtusifolia (Lam.) Griseb.; Centrostachys aspera (L.) Standl.; Centrostachys indica (L.) Standl.]
- Alternanthera ficoidea (L.) P. Beauv. var. bettzickiana (Regel) Backer, Sanguinaria, † Waif (Reed 41012 MO) [Alternanthera bettzickiana (Regel) G. Nicholson]

Alternanthera philoxeroides (Mart.) Griseb., Alligatorweed, † (Hall \& Anderson JH2014.09.16-07 TAWES)

- Alternanthera pungens Kunth, Khakiweed, † Waif (Reed $32830 \mathrm{MO})$

[Alternanthera repens (L.) Link]

- Alternanthera sessilis (L.) R. Br. ex DC., Sessile Chaff-flower, † Waif (Reed $41736 \mathrm{MO}$ )

Amaranthus albus L., White Pigweed, † (Ebert \& Holt 135EH DOV)

[Amaranthus graecizans L. var. pubescens Uline \& W. L. Bray]

Amaranthus blitoides S. Watson, Matweed Amaranth, $\dagger$ (Fredholm 2116 US)

Amaranthus blitum L., Purple Amaranth, † (Longbottom 2735 DOV)

[Amaranthus lividus L.]

Amaranthus cannabinus (L.) J. D. Sauer, Waterhemp Pigweed (Longbottom 9973 DOV)

[Acnida cannabina L.]

- Amaranthus cruentus L., Red Amaranth, † Waif (Reed 41165 $\mathrm{MO})$

- Amaranthus deflexus L., Large-fruited Amaranth, † Waif (Reed 32823 US)

Amaranthus bybridus L., Smooth Amaranth (Killip 40186 US)

- Amaranthus palmeri S. Watson, Palmer's Amaranth, † Waif (Reed 32979 US)

Amaranthus pumilus Raf., Seabeach Amaranth, S1/G2, LT (Hamilton s.n. DOV)

Amaranthus retroflexus L., Red-root Amaranth, †? (Dill 129 DOV)

Amaranthus spinosus L., Spiny Amaranth, † (Longbottom 4279 DOV)

- Atriplex elegans (Moq.) D. Dietr. var. elegans, Wheel-scale Saltbush, † Waif Reed (1964)

[Atriplex thornberi (M. E. Jones) Standl.]

- Atriplex hortensis L., Giant Orache, † Waif Reed (1964) [Atriplex nitens Schkuhr]

- Atriplex littoralis L., Grassleaf Orache, † Waif Reed (1964)

Atriplex mucronata Raf., Crested Saltbush, S1S2/G5 (Benedict 3233 US)

[Atriplex arenaria Nutt.; A. cristata Humb. \& Bonpl. ex Willd.]

Atriplex patula L., Halberd-leaf Orache, $†$ (McAvoy 3192 DOV) [Atriplex patula var. japonica Level.]

Atriplex prostrata Bouchér ex DC., Creeping Saltbush, † (Longbottom 4573 DOV)

[Atriplex hastata L.]

Atriplex rosea L., Tumbling Orache, $†$ (Plitt s.n. US)

Bassia birsuta (L.) Asch., Hairy Smotherweed, † (Higgins s.n. DOV) 
Bassia scoparia (L.) A. J. Scott, Summer Cypress, † (Buck s.n. MARY)

[Kochia scoparia (L.) Schrad.]

- Beta vulgaris L. ssp. vulgaris, Common Beet, † Waif (Norton s.n. MARY)

Celosia argentea L., Silvery Cock's Comb, † (Norton s.n. MARY) [Celosia margaritacea L.]

Chenopodiastrum murale (L.) S. Fuentes-B., Uotila, \& Borsch, Nettle-leaf Goosefoot, † (Otis s.n. DOV)

[Chenopodium murale L.]

Chenopodiastrum simplex (Torr.) S. Fuentes-B., Uotila, \& Borsch, Giant-seed Goosefoot, S2/G5 (Canby s.n. DOV) [Chenopodium gigantospermum Aellen; C. hybridum L. C. simplex (Torr.) Raf.]

Chenopodium album L., White Goosefoot, $\ddagger$ (Hotchkiss 5708 US)

[Chenopodium album var. missouriense (Aellen) Bassett \& Crompton; C. amaranticolor H. J. Coste \& Reyn.; C. giganteum D. Don; C. lanceolatum Muhl. ex Willd.; C. missouriense Aellen; C. paganum Rchb.]

Chenopodium berlandieri Moq., Pit-seed Goosefoot (Vasey s.n. US)

[Chenopodium berlandieri var. boscianum (Moq.) Wahl; C. berlandieri var. bushianum (Aellen) Cronquist; C. berlandieri var. macrocalycium (Aellen) Cronquist; C. bushianum Aellen]

- Chenopodium bonus-henricus L., Good King Henry, † Waif (Longbottom 406 DOV)

- Chenopodium carnosulum Moq., Ridged Goosefoot, † Waif Reed (1964)

Chenopodium glaucum L. var. glaucum, Oakleaf Goosefoot, $\dagger$ (Piper s.n. US)

Chenopodium leptophyllum (Moq.) Nutt. ex S. Watson, Narrowleaf Goosefoot, †? (Vasey s.n. US)

- Chenopodium macrospermum Hook. f., Large-Seed Goosefoot, $†$ Waif Reed (1964)

[Chenopodium farinosum Standl.]

- Chenopodium opulifolium Schrad. ex DC., Seaport Goosefoot, $†$ Waif Reed (1964)

[Chenopodium viride L.]

Chenopodium polyspermum L., Many-seed Goosefoot, † (Otis s.n. DOV)

[Chenopodium polyspermum var. acutifolium (Sm.) Gaudich.; C. polyspermum var. obtusifolium Gaudich.]

Chenopodium pratericola Rydb., Desert Goosefoot, $\dagger$ (Norton s.n. MARY)

[Chenopodium desiccatum A. Nelson var. leptophylloides (Murr) Wahl]

- Chenopodium rubrum L., Coast-blite Goosefoot-see Excluded Flora section.

[Oxybasis rubra (L.) S. Fuenes-B., Uotila, \& Borsch]
Chenopodium standleyanum Aellen, Standley's Goosefoot, S2S3/G5 (Norton s.n. MARY)

- Chenopodium urbicum L., City Goosefoot-see Excluded Flora section.

[Oxybasis urbica (L.) S. Fuenes-B., Uotila, \& Borsch]

- Chenopodium vulvaria L., Stinking Goosefoot, † Waif Reed (1964)

Cycloloma atriplicifolium (Spreng.) J. M. Coult., Winged Pigweed, $†$ (Cohen 12 MARY)

Dysphania ambrosioides (L.) Mosyakin \& Clemants, Wormseed Goosefoot, †? (Hotchkiss 5508 US)

[Chenopodium ambrosioides L.]

Dysphania botrys (L.) Mosyakin \& Clemants, Jerusalem Oak, † (Norton s.n. MARY)

[Chenopodium botrys L.]

- Dysphania plantaginella F. Muell., Dwarf Flower, † Waif Reed (1964)

[Chenopodium plantaginellum (F. Muell.) Aellen]

Dysphania pumilio (R. Br.) Mosyakin \& Clemants, Clammy Goosefoot, $\dagger$ (Beste s.n. MARY)

Froelichia floridana (Nutt.) Moq. var. floridana, Florida Cottonweed, † (Longbottom \& Knapp 7654 DOV)

Froelichia gracilis (Hook.) Moq., Slender Cottonweed, † (Longbottom 11861 DOV)

Gomphrena globosa L., Globe Amaranth, † (Brown s.n. MARY)

- Gomphrena serrata L., Arrasa Con Todo, † Waif (Reed 43645 $\mathrm{MO})$

[Gomphrena celosioides Martens]

- Guilleminea densa (Humb. \& Bonpl. ex Schult.) Moq. var. aggregata Uline \& W. L. Bray, Cottonflower, † Waif (Reed $32709 \mathrm{MO})$

- Hermbstaedtia odorata (Burch. ex Moq.) T. Cooke, Guineaflower, † Waif (Reed $45791 \mathrm{MO}$ )

[Hermbstaedtia elegans Moq.]

Iresine rhizomatosa Standl., Eastern Bloodleaf, S1/G5 (Lea 987 TAWES)

- Polycnemum majus A. Braun, Giant Needleleaf, † Waif Reed (1964)

Salicornia ambigua Michx., Woody Glasswort (Higgins s.n. MARY)

[Sarcocornia ambigua (Michx.) M. Á. Alonso \& M. B. Crespo; S. perennis (Mill.) A. J. Scott]

Salicornia bigelovii Torr., Dwarf Glasswort (Hill 15591 US)

Salicornia virginica L., Virginia Glasswort (Fosberg 18489 US) [Salicornia depressa Standl.; S. europaea L.] 
Salsola kali L. ssp. kali, Russian Thistle, † (Leonard 6000 US)

Salsola kali L. ssp. pontica (Pallas) Mosyakin, Russian Thistle, † (Smith s.n. US)

Salsola tragus L., Prickly Russian Thistle, † (Leonard 5674 US) [Salsola kali L. var. tenuifolia Moq.; S. pestifer A. Nelson]

Suaeda linearis (Elliott) Moq., Narrowleaf Seepweed, S3/G5 (Lea 836 TAWES)

[Dondia linearis (Elliott) Heller]

Suaeda maritima (L.) Dumort., Herbaceous Seepweed (Hamilton 240 Assateague Island National Seashore Herbarium)

\section{AMARYLLIDACEAE}

(Amaryllis Family)

- Allium allegheniense Small, Allegheny Onion-see Excluded Flora section.

Allium canadense L. var. canadense, Wild Onion (Bartsch s.n. US) Allium cernuum Roth, Nodding Onion (Bartsch s.n. US)

- Allium sativum L., Garlic-see Excluded Flora section. Allium schoenoprasum L., Chives, † (Wright s.n. MARY) Allium tricoccum Aiton, Ramps (Beyersdorfer 8 US) Allium vineale L., Field Garlic, ‡ (Fleming 2223 US) Galanthus nivalis L., Snowdrop, † (Steury 990324 US)

Lencojum aestivum L., Summer Snowflake, † (Heimer 12 MARY)

Narcissus jonquilla L., Jonquil, † (Chanoski 046 BALT) Narcissus poeticus L., Poet's Narcissus, † (Standley 11302 US) Narcissus pseudonarcissus L., Daffodil, † (Standley 11340 US)

Zephyranthes atamasca (L.) Herb., Atamasco Lily, S1/G4G5 (McAvoy 1037 DOV)

\section{ANACARDIACEAE}

(Sumac/Cashew Family)

- Cotinus coggygria Scop., Smoketree-see Excluded Flora section.

Rhus aromatica Aiton var. aromatica, Fragrant Sumac (Longbottom 11861 DOV)

[Rhus trilobata Nutt.]

Rhus copallinum L. var. copallinum, Winged Sumac (Dill 507 DOV)

[Rhus copallinum var. latifolia Engl.; R. copallinum var. leucantha (Jacq.) DC.]

Rhus glabra L., Smooth Sumac (McAvoy 6289 DOV)

Rhus typhina L., Staghorn Sumac (Tatnall 4998 DOV)

[Rhus hirta (L.) Sudw.]

Toxicodendron pubescens Mill., Eastern Poison Oak (McAvoy 1760 DOV)

[Rhus toxicodendron L.; Toxicodendron toxicarium (Salisb.) Gillis; T. toxicodendron (L.) Britton]

Toxicodendron radicans (L.) Kuntze, Eastern Poison Ivy (Longbottom 8719 DOV)

[Rhus radicans $\mathrm{L}$.]
- Toxicodendron rydbergii (Small ex Rydb.) Greene, Northern Poison Oak-see Excluded Flora section.

Toxicodendron vernix (L.) Kuntze, Poison Sumac (Strong \& Simmons 3474 DOV)

[Rhus vernix L.]

ANNONACEAE

(Custard Apple Family)

Asimina triloba (L.) Dunal, Pawpaw (Dill 157 DOV)

APIACEAE [Umbelliferae] (Carrot/Parsley Family)

Aegopodium podagraria L., Bishop's Goutweed, † (Longbottom 3130 MARY)

Anethum graveolens L., Dill, † (Hill 16836 MARY)

Angelica atropurpurea L., Great Angelica, SH/G5 (Turpin 307 US)

Angelica triquinata Michx., Filmy Angelica, S1/G5 (Reynard s.n. MARY)

[Angelica curtisii Buckley]

Angelica venenosa (Greenway) Fernald, Hairy Angelica (Tatnall 4667 DOV)

[Angelica villosa (Walter) Britton, Sterns, \& Poggenb.]

Anthriscus cancalis M. Bieb., Bur Chervil, † (Longbottom 15010 DOV)

Anthriscus sylvestris (L.) Hoffm., Wild Chervil, † (Terrell \& Terrell 5590 MARY)

Apium graveolens L., Wild Celery, † (Norton s.n. MARY)

- Bifora testiculata (L.) Spreng., European Bishop, † Waif Reed (1964)

- Bupleurum lancifolium Hornem., Lanceleaf Thoro Wax, † Waif Reed (1964)

[Bupleurum protractum Link \& Hoffm.]

- Bupleurum odontites L., Narrowleaf Thoro Wax, † Waif Reed (1964)

- Bupleurum rotundifolium L., Roundleaf Thoro Wax, $†$ Waif (Miller s.n. US)

Carum carvi L., Common Caraway, † (Longbottom 17225 NY)

Centella asiatica (L.) Urb., Spadeleaf, S3/G5 (McAvoy 1137 DOV)

[Centella erecta (L.) Fernald]

Chaerophyllum procumbens (L.) Crantz var. procumbens, Spreading Chervil (Naczi 8052 DOV)

Chaerophyllum procumbens (L.) Crantz var. shortii Torr. \& A. Gray, Wild Chervil (Fogg 1927 PH)

Chaerophyllum tainturieri Hook., Hairy-fruit Chervil (Longbottom 6115 DOV)

[Chaerophyllum floridanum Bush] 
Cicuta bulbifera L., Bulb-bearing Water Hemlock, S1/G5 (Leonard 1367 US)

Cicuta maculata L. var. maculata, Spotted Water Hemlock (Longbottom 9963 DOV)

[Cicuta curtissii J. M. Coult. \& Rose; C. maculata var. bolanderi (S. Watson) G. A. Mulligan]

Conium maculatum L., Poison Hemlock, † (Longbottom 6640 DOV)

- Coriandrum sativum L., Coriander, † Waif (Norton s.n. MARY)

Cryptotaenia canadensis (L.) DC., Canadian Honewort (Knapp \& Frye 1408 DOV)

[Deringa canadensis (L.) Kuntze]

- Cyclospermum leptophyllum (Pers.) Sprague ex Britton \& P. Wilson, Marsh Parsley, † Waif Reed (1964)

[Apium leptophyllum (Pers.) F. Muell.]

Daucus carota L., Wild Carrot, $\ddagger$ (Longbottom 11684 DOV)

Erigenia bulbosa (Michx.) Nutt., Harbinger-of-spring, S3/G5 (Longbottom 10904 DOV)

Eryngium aquaticum L., Marsh Rattlesnake-master (Knapp 2310 DOV)

Eryngium campestre L., Snakeroot Coyote Thistle, † (Taylor s.n. US)

Eryngium yuccifolium Michx. var. yuccifolium, Rattlesnakemaster, $/$ / SH/G5 (Shoemaker s.n. US)-historic at a single native location but now rarely found as an accidental introduction in warm-season grass mixes

- Falcaria vulgaris Bernh., Sickleweed-see Excluded Flora section.

[Falcaria sioides Asch.]

Foeniculum vulgare Mill., Sweet Fennel, † (Walker 3863 US)

Heracleum mantegazzianum Sommier \& Levier, Giant Hogweed, $\ddagger$ (Heimer Photo Voucher MARY)

Heracleum maximum W. Bartram, Cow Parsnip, S3/G5 (Smith 5326 US)

[Heracleum lanatum Michx.]

Imperatoria ostruthium L., Masterwort, $†$ (Engh s.n. MARY) [Peucedanum ostruthium (L.) W. D. J. Koch]

Ligusticum canadense (L.) Britton, Nondo Lovage, SH/G4 (Baltars 192 US)

Lilaeopsis chinensis (L.) Kuntze, Eastern Lilaeopsis (Steury 990710.6 US)
- Oenanthe aquatica (L.) Poir, Fineleaf Waterdropwort-see Excluded Flora section.

Oenanthe javanica (Blume) DC., Java Waterdropwort, † (Strong et al. 3566 US)

Osmorbiza claytonii (Michx.) C. B. Clarke, Hairy Sweet-cicely (Smith 5068 US)

[Washingtonia claytonii (Michx.) Britton]

Osmorhiza longistylis (Torr.) DC., Smooth Sweet-cicely (Knapp 525 DOV)

[Washingtonia longistylis (Torr.) Britton]

Oxypolis rigidior (L.) Raf., Stiff Cowbane (Knapp 2314 DOV) [Oxypolis rigidior var. longifolia (Pursh) Britton; O. turgida Small]

Pastinaca sativa L., Wild Parsnip, † (Hill 11819 MARY)

- Petroselinum crispum (Mill.) Nyman ex A. W. Hill, Garden Parsley-see Excluded Flora section.

Pimpinella saxifraga L., Burnet-saxifrage (Baltars 3556 MARY)

Ptilimnium capillaceum (Michx.) Raf., Mock Bishopweed (Shetler et al. s.n. US)

Ptilimnium nodosum (Rose) Mathias, Harperella, S1/G2, LE (Boone s.n. TAWES)

[Harperella fluviatilis Rose; H. nodosa Rose; Ptilimnium fluviatile (Rose) Mathias]

Sanicula canadensis L. var. canadensis, Canadian Black-snakeroot (Longbottom $6171 \mathrm{DOV}$ )

[Sanicula floridana E. P. Bicknell]

Sanicula marilandica L., Maryland Black-snakeroot, S3/G5 (McAvoy 6616 DOV)

Sanicula odorata (Raf.) K. M. Pryer \& L. R. Phillippe, Clustered Black-snakeroot (Hirst s.n. DOV)

[Sanicula gregaria E. P. Bicknell]

Sanicula trifoliata E. P. Bicknell, Large-fruited Sanicle, S3/G4 (Baltars 4133 US)

- Scandix pecten-veneris L., Shepherds-needle-see Excluded Flora section.

- Seseli libanotis (L.) W. D. J. Koch, Moon-carrot, † Waif (Norton s.n. US)

Sium suave Walter, Hemlock Water Parsnip (Shetler et al. s.n. US)

- Spermolepis inermis (Nutt. ex DC.) Mathias \& Constance, Red River Scaleseed-see Excluded Flora section. [Spermolepis patens (Nutt. ex DC.) B. L. Rob.]

Taenidia integerrima (L.) Drude, Yellow Pimpernel (Knapp 2958 DOV) 
Taenidia montana (Mack.) Cronquist, Mountain Parsley, - S2/G3 (Reveal \& Broome 5260 MARY)

[Pseudotaenidia montana Mack.]

Thaspium barbinode (Michx.) Nutt., Hairy-joint Meadow Parsnip (Naczi 11049 DOV)

Thaspium trifoliatum (L.) A. Gray var. trifoliatum, Purple Meadow Parsnip, S1/G5T5 (Baltars 467 MARY)

[Thaspium trifoliatum var. aureum Britton; T. trifoliatum var. flavum S. F. Blake]

Tiedemannia canbyi (J. M. Coult. \& Rose) Fiest \& S. R. Downie, Canby's Dropwort, S1/G2 LE (Samson \& Canick s.n. TAWES)

[Oxypolis canbyi J. M. Coult. \& Rose) Fernald]

- Torilis arvensis (Huds.) Link, Spreading Hedge Parsley, † Waif (Sollers s.n. US)

- Torilis japonica (Houtt.) DC., Erect Hedge Parsley, † Waif Reed (1964)

- Torilis nodosa (L.) Gaertn., Knotted Hedge Parsley, † Waif (Foreman s.n. US)

[Caucalis nodosa (L.) Huds.]

Zizia aptera (A. Gray) Fernald, Heartleaf Alexanders (Boone s.n. TAWES)

[Zizia cordata W. D. J. Koch ex DC.]

Zizia aurea (L.) W. D. J. Koch, Golden Alexanders, S3/G5 (Tatnall 4824 DOV)

Zizia trifoliata (Michx.) Fernald, Meadow Alexanders (McAvoy $6743 \mathrm{DOV})$

[Zizia arenicola Rose; Z. bebbii (J. M. Coult. \& Rose) Britton]

\section{APOCYNACEAE}

(Dogbane Family)

- Amsonia tabernaemontana Walter var. tabernaemontana, Eastern Blue Dogbane-see Excluded Flora section. [Amsonia amsonia (L.) Britton]

Apocynum androsaemifolium L., Spreading Dogbane (Baltars 143 MARY)

Apocynum cannabinum L., Clasping-leaf Dogbane (McAvoy $1672 \mathrm{DOV})$

[Apocynum sibiricum Jacq.]

Asclepias amplexicaulis Sm., Clasping Milkweed (McAvoy 2527 DOV)

[Asclepias obtusifolia Michx.]

Asclepias exaltata L., Poke Milkweed (Fessenden s.n. US) [Asclepias phytolaccoides Pursh]

Asclepias incarnata L. ssp. incarnata, Western Swamp Milkweed (Holmes s.n. US)

Asclepias incarnata L. ssp. pulchra (Ehrh. ex Willd.) Pers., Eastern Swamp Milkweed (Smith 5408 US)
Asclepias lanceolata Walter, Smooth Orange Milkweed, S1/G5 (Smith s.n. DOV)

- Asclepias linearis Scheele, Slim Milkweed—see Excluded Flora section.

- Asclepias longifolia Michx., Longleaf Milkweed—see Excluded Flora section.

[Acerates floridana (Lam.) Hitchc.; A. longifolia (Michx.) Elliott]

Asclepias purpurascens L., Purple Milkweed, S2/G5 (Steury 980612.1 US)

Asclepias quadrifolia Jacq., Fourleaf Milkweed (Roller 515 US)

Asclepias rubra L., Red Milkweed, S1/G4G5 (McAvoy 1128 DOV)

Asclepias syriaca L., Common Milkweed (Longbottom 11611 DOV)

Asclepias tuberosa L. ssp. tuberosa, Butterfly Milkweed (McAvoy 2697 DOV)

Asclepias variegata L., White Milkweed (McAvoy 5482 DOV)

Asclepias verticillata L., Whorled Milkweed, S3/G5 (Isanogle 30 US)

Asclepias viridiflora Raf., Green Milkweed (McAvoy 5928 DOV) [Acerates viridiflora (Raf.) Pursh ex Eaton]

Cynanchum laeve (Michx.) Pers., Honeyvine (Longbottom 11748 DOV)

[Ampelamus albidus (Nutt.) Britton]

Gonolobus suberosus (L.) R. Br. var. suberosus, Angular-fruit Milkvine, S2/G5 (Steury 050716.2 US)

[Gonolobus gonocarpos (Walter) L. M. Perry; Matelea gonocarpos (Walter) Shinners]

Matelea carolinensis (Jacq.) Woodson, Carolina Anglepod, - S2S3/G4 (McAvoy 1673 DOV)

[Vincetoxicum carolinense (Jacq.) Britton; V. hirsutum (Michx.) Britton]

- Matelea decipiens (Alexander ex Small) Woodson, Old-field Milkvine-see Excluded Flora section.

Matelea obliqua (Jacq.) Woodson, Climbing Milkweed, S1S2/ G4? (Killip 32116 US)

Thyrsanthella difformis (Walter) Pichon, Climbing Dogbane, - S1/G4G5 (McAvoy 1121 DOV) [Trachelospermum difforme (Walter) A. Gray]

Vinca major L., Largeleaf Periwinkle, ‡ (Longbottom 7193 DOV) Vinca minor L., Lesser Periwinkle, $\ddagger$ (Redmond 232 DOV)

Vincetoxicum nigrum (L.) Moench, Black Swallow-wort, $\ddagger$ (Baltars 3763 MARY)

[Cynanchum louiseae Kartesz \& Gandhi; C. nigrum (L.) Pers.]

AQUIFOLIACEAE

(Holly Family)

Ilex crenata Thunb., Japanese Holly, $\ddagger$ (Redman 9549 BALT) Ilex decidua Walter, Deciduous Holly, S2/G5 (Terrell 3886 US) 
Ilex glabra (L.) A. Gray, Inkberry (Killip 43318 US)

Ilex laevigata (Pursh) A. Gray, Smooth Winterberry Holly (Strong 1858 US)

Ilex montana Torr. \& A. Gray, Mountain Holly (Brown s.n. BALT)

[Ilex ambigua (Michx.) Torr. var. montana (Torr. \& A. Gray) H. E. Ahles; I. ambigua var. monticola (A. Gray) Wunderlin \& Poppleton; I. monticola A. Gray; I. monticola var. mollis (A. Gray) Britton]

Ilex mucronata (L.) M. Powell, Savol. \& S. Andrew, Catberry, - S3/G5 (McClurkin 2008-042 BALT)

[Nemopanthus mucronatus (L.) Trel.]

Ilex opaca Aiton, American Holly (Longbottom 10808 DOV)

Ilex verticillata (L.) A. Gray, Common Winterberry (McAvoy 1046 DOV)

ARACEAE

(Arum Family)

Arisaema dracontium (L.) Schott, Green Dragon (McAvoy 5462 DOV)

[Muricauda dracontium (L.) Small]

Arisaema pusillum (Peck) Nash, Small Jack-in-the-Pulpit (McAvoy 6873 DOV)

[Arisaema triphyllum ssp. pusillum (Peck) Huttleston]

Arisaema stewardsonii Britton, Bog Jack-in-the-Pulpit (Henry o Buker s.n. CM)

[Arisaema triphyllum ssp. stewardsonii (Britton) Huttleston]

Arisaema triphyllum (L.) Schott, Common Jack-in-the-Pulpit (Knapp 2372 DOV)

[Arisaema atrorubens Blume]

Arum italicum Mill. ssp. italicum, Italian Arum, † (Hill s.n. MARY)

Calla palustris L., Wild Calla, S1/G5 (Longbottom et al. 2046 MARY)

Landoltia punctata (G. Mey.) Les \& D. J. Crawford, Dotted Duckmeat (Steury 990626.4 US)

[Spirodela punctata (G. Mey.) C. H. Thomps.]

Lemna aequinoctialis Welw., Lesser Duckweed (Solommon 350 MARY)

Lemna gibba L., Fat Duckweed, † (Reveal 7913 MARY)

Lemna minor L., Common Duckweed (Baltars 1136 US)

Lemna obscura (Austin) Daubs, Little Duckweed (Tidestrom 296 US)

Lemna perpusilla Torr., Tiny Duckweed (Killip 36635 US)

Lemna trisulca L., Star Duckweed, S1/G5 (Uhler s.n. US)

Lemna turionifera Landolt, Duckweed (Reveal et al. 7779 MARY)

Lemna valdiviana Phil., Pale Duckweed (Reveal 5583 MARY)
Orontium aquaticum L., Golden Club (Knapp \& Stango III 01-02 DOV)

Peltandra virginica (L.) Raf. ex Schott, Tuckahoe (Longbottom $6164 \mathrm{DOV})$

Pinellia ternata (Thunb.) Makino ex Breitenb., Pinellia, † (Minor s.n. CM)

Pistia stratiotes L., Water Lettuce, $\ddagger($ Knapp 2342 TAWES)

Spirodela polyrbiza (L.) Schleid., Greater Duckweed (Beyersdorfer 234 US)

Symplocarpus foetidus (L.) Salisb., Skunk Cabbage (Gribbon 4 DOV)

- Wolffia borealis (Engelm.) Landolt \& Wildi, Northern Waterwheel-see Excluded Flora section. [Wolffia punctata auct. non Griseb.]

Wolffia brasiliensis Wedd., Brazilian Waterwheel (Steury 990513.1 US)

[Wolffia papulifera C. H. Thomps.; W. punctata Griseb.]

Wolffia columbiana H. Karst., Columbian Waterwheel (Hotchkiss 7729 US)

Wolffiella gladiata (Hegelm.) Hegelm., Mudmidget (Longbottom 8148 MARY)

[Wolffia floridana (J. D. Smith) C. H. Thomps.]

ARALIACEAE

(Ginseng Family)

Aralia elata (Miq.) Seem., Japanese Angelica Tree, † (Hubick s.n. Photo Voucher MPA)

Aralia hispida Vent., Bristly Sarsaparilla, S1/G5 (Thompson s.n. TAWES)

Aralia nudicaulis L., Wild Sarsaparilla (McAvoy 983 DOV)

Aralia racemosa L., American Spikenard, S2S4/G4G5 (Adams of Hopkins $897 \mathrm{PH})$

Aralia spinosa L., Hercules Club (Otis s.n. DOV)

Eleutherococcus sieboldianus (Makino) Koidz., Five-leaf Aralia, † (Longbottom $26934 \mathrm{NY)}$

Hedera helix L., English Ivy, $\ddagger$ (Ditman s.n. MARY)

Hedera hibernica (G. Kirchn.) Bean, Irish Ivy, $\ddagger$ (Longbottom 10828 DOV)

Hydrocotyle americana L., American Water-pennywort (Pollard 471 US)

Hydrocotyle ranunculoides L., Floating Marsh-pennywort (Killip 36649 US)

Hydrocotyle sibthorpioides Lam., Lawn Marsh-pennywort, † (Smith 4806 US) 
Hydrocotyle umbellata L., Many-flowered Marsh-pennywort (Higgins s.n. MARY)

Hydrocotyle verticillata Thunb., Whorled Marsh-pennywort (Shull 325 US)

[Hydrocotyle australis J. M. Coult. \& Rose; H. canbyi J. M. Coult. \& Rose; H. prolifera Kellogg; H. verticillata var. triradiata (A. Rich.) Fernald]

Kalopanax septemlobus (Thunb.) Koidz., Castor Aralia, † (Steury 021008.3 US)

Panax quinquefolius L., American Ginseng, S2S3/G3G4 (McAvoy 4494 DOV)

Panax trifolius L., Dwarf Ginseng (Knapp 511 DOV)

ARECACEAE [Palmae]

(Palm Family)

- Phoenix dactylifera L., Date Palm, † Waif Reed (1964)

\section{ARISTOLOCHIACEAE}

(Birthwort Family)

Aristolochia clematitis L., Birthwort, † (Plitt 312 US)

Asarum canadense L., Wild Ginger (Knapp 2399 APSC)

Asarum reflexum E. P. Bicknell, Reflexed Wild Ginger (Maxon 318 US)

Asarum virginicum L., Virginia Heartleaf, S1/G4 (Councill s.n. MARY)

[Hexastylis virginica (L.) Small)

Endodeca serpentaria (L.) Raf., Virginia Snakeroot (McAvoy $1012 \mathrm{DOV})$

[Aristolochia serpentaria L.]

Isotrema macrophyllum (Lam.) C. F. Reed, Pipevine, S2/G5 (Boone s.n. TAWES)

[Aristolochia durior Hill; A. macrophylla Lam.]

\section{ASPARAGACEAE}

(Asparagus Family)

- Agave virginica L., Eastern False Aloe-see Excluded Flora section.

[Manfreda tigrina (Engelm.) Small; M. virginica (L.) Rose; Polianthes virginica (L.) Shinners]

Asparagus officinalis L., Asparagus, † (Lombardo \& Windler $3621 \mathrm{DOV})$

Camassia scilloides (Raf.) Cory, Wild Hyacinth, † (Longbottom et al. 3463 MARY)

Convallaria majalis L., European Lily of the Valley, $†$ (Huston s.n. MARY)

- Hosta lancifolia (Thunb.) Engelm., Narrowleaf Plantain Lily — see Excluded Flora section.
Hosta ventricosa (Salisb.) Stearn, Blue Plantain Lily, † (Engh s.n. MARY)

- Liriope graminifolia (L.) Baker, Lilyturf-see Excluded Flora section.

- Liriope muscari (Decne.) L. H. Bailey, Big Blue Lilyturf-see Excluded Flora section.

Liriope spicata (Thunb.) Lour., Creeping Liriope, † (Knapp \& Wixted 4352 MARY)

Maianthemum canadense Desf., Canada Mayflower (Fessenden 5223 US)

Maianthemum racemosum (L.) Link, False Solomon's Seal (Beyersdorfer 33 US)

[Smilacina racemosa (L.) Desf.]

Maianthemum stellatum (L.) Link, Starry False Solomon's Seal, - S2/G5 (Barger s.n. MARY)

[Smilacina stellata (L.) Desf.]

Muscari botryoides (L.) Mill., Compact Grape Hyacinth, † (Standley 11290 US)

Muscari neglectum Guss. ex Ten., Grape Hyacinth, † (Pollard 105 US)

[Muscari racemosum (L.) Mill.]

Ornithogalum nutans L., Drooping Star-of-Bethlehem, $\ddagger$ (Beyersdorfer 26 US)

Ornithogalum umbellatum L., Star-of-Bethlehem, $\ddagger$ (Beyersdorfer 84 US)

Polygonatum biflorum (Walter) Elliott var. biflorum, Small Solomon's Seal (Beyersdorfer 25 US)

Polygonatum biflorum (Walter) Elliott var. commutatum (Schult. f.) Morong, Large Solomon's Seal (Hall JH2015.05.20-1 MARY)

[Polygonatum canaliculatum auct. non (Muhl. ex Willd.) Pursh; P. commutatum (Schult. f.) A. Dietr.]

Polygonatum pubescens (Willd.) Pursh, Hairy Solomon's Seal (Hill 11146 MARY)

Yucca filamentosa L., Curlyleaf Yucca (Benedict s.n. US)

Yucca flaccida Haw., Weakleaf Yucca, † (Harms Photo Voucher MPA)

[Yucca smalliana Fernald]

\section{ASPHODELACEAE}

(Asphodel/Daylily Family)

Hemerocallis fulva (L.) L., Orange Daylily, $\uparrow$ (Beyersdorfer 76 US)

Hemerocallis lilioasphodelus L., Yellow Daylily, † (McKinney 34 Frostburg State University Herbarium)

[Hemerocallis flava L.]

ASTERACEAE [Compositae]

(Aster Family)

Achillea borealis Bong., American Yarrow (Bell s.n. US) 
- Achillea millefolium L., Common Yarrow-see Excluded Flora section. [Achillea aspleniifolia Vent.; A. millefolium var. occidentalis DC.; A. occidentalis (DC.) Raf. ex Rydb.]

- Achillea ptarmica L., Sneezewort, † Waif (s. coll., s.n. MARY)

Ageratina altissima (L.) R. M. King \& H. Rob. var. altissima, White Snakeroot (McAvoy 1322 DOV)

[Eupatorium ageratoides L. f.; E. rugosum Houtt.]

Ageratina aromatica (L.) Spach, Lesser Snakeroot (McAvoy 362 DOV)

[Eupatorium aromaticum L.; E. latidens Small]

Ageratum conyzoides L., Yerba de Cabrio, † Waif Reed (1964)

Ambrosia artemisiifolia L., Annual Ragweed (Hitchcock 15770 US)

[Ambrosia artemisiifolia var. elatior (L.) Descourt.; A. artemisiifolia var. paniculata (Michx.) Blank.; A. elatior L.; A. glandulosa Scheele; A. monophylla (Walter) Rydb.]

- Ambrosia bidentata Michx., Lanceleaf Ragweed, † Waif (Pladeck s.n. US)

Ambrosia trifida L. var. trifida, Great Ragweed (Hitchcock 15799 US)

Anaphalis margaritacea (L.) Benth. \& Hook. f., Pearly Everlasting, S3/G5 (Topping s.n. US)

[Anaphalis margaritacea var. intercedens Hara]

Antennaria howellii Greene, Howell's Pussytoes (McAvoy 5460 DOV)

[Antennaria howellii ssp. canadensis (Greene) R. J. Bayer; A. howellii ssp. neodioica (Greene) R. J. Bayer; A. howellii ssp. petaloidea (Fernald) R. J. Bayer; A. neglecta Greene var. attenuata (Fernald) Cronquist Greene; A. neodioica]

Antennaria neglecta Greene, Field Pussytoes (Chickering s.n. US)

Antennaria parlinii Fernald, Parlin's Pussytoes (Arsène 1406 US)

[Antennaria plantaginifolia Hook. var. arnoglossa (Greene) Cronquist]

Antennaria plantaginifolia Hook., Plantain-leaf Pussytoes (Reveal \& Broome 5245 MARY)

[Antennaria caroliniana Rydb.; A. fallax Greene]

Antennaria solitaria Rydb., Single-head Pussytoes, S2/G5 (McAvoy 966 DOV)

Antennaria virginica Stebbin, Shale Barren Pussytoes (Bartlett 1916 CM)

Anthemis arvensis L., Corn Chamomile, † (Killip 31245 US)

Anthemis cotula L., Mayweed, † (Standley 11799 US)

[Maruta cotula (L.) DC.]

Arctium lappa L., Greater Burdock, † (Norton s.n. MARY) Arctium minus (Hill) Bernh., Lesser Burdock, † (Blake s.n. US)

- Arctium tomentosum Mill., Woolly Burdock, † Waif (Baltars 2675 US)
Arnica acaulis Britton, Sterns, \& Poggenb., Leopard's-bane, - S1/G4 (Fessenden \& Baltars 1045 US)

Arnoglossum atriplicifolium (L.) H. Rob., Pale Indian-plantain (McAvoy 1154 DOV)

[Cacalia atriplicifolia L.; Mesadenia atriplicifolia (L.) Raf.]

Arnoglossum reniforme (Hook.) H. Rob., Great Indian-plantain, - SH/G5 (Benedict 3610 US)

[Arnoglossum muehlenbergii (Sch. Bip.) H. Rob.; Cacalia muehlenbergii (Sch. Bip.) Fernald]

Artemisia abrotanum L., Southern Wormwood, † (Norton \& Chrysler s.n. MARY), perhaps a waif

Artemisia absinthium L., Absinthium, † (Baltars 717 US)

Artemisia annua L., Annual Wormwood, † (Blake s.n. US)

- Artemisia biennis Willd., Biennial Wormwood-see Excluded Flora section.

- Artemisia ludoviciana Nutt., White Sagebrush, † Waif (Baltars 718 US)

- Artemisia pontica L., Roman Wormwood-see Excluded Flora section.

- Artemisia scoparia Waldst. \& Kit., Red-stem Wormwood, † Waif Reed (1964)

Artemisia stelleriana Besser, Hoary Sagebrush, $†$ (Anderson \& Rappleye s.n. MARY)

Artemisia vulgaris L., Common Wormwood, $\ddagger$ (Baltars 748 US)

Aster tataricus L., Tartarian Aster, † (Riefner 80-414 MARY)

Baccharis halimifolia L., Eastern Baccharis (Leonard 611 US)

- Babia schaffneri S. Watson, Schaffner's Bahia, † Waif (Reed 32787 US)

Bellis perennis L., Lawn Daisy, † (Simmons s.n. MARY)

Bidens aristosa (Michx.) Britton, Tickseed Beggar-ticks, $\dagger$ (Allard 7412 US)

[Bidens involucrata (Nutt.) Britton]

- Bidens beckii (Torr. ex Spreng.) Greene, Beck's Watermarigold-see Excluded Flora section.

[Megalodonta beckii (Torr. ex. Spreng.) Greene]

Bidens bidentoides (Nutt.) Britton, Maryland Bur-marigold, - S3.1/G3G4 (Shull 399 US)

[Bidens bidentoides var. mariana Sherff]

Bidens bipinnata L., Spanish Needles (Hitchcock 15823 US)

Bidens cernua L., Nodding Beggar-ticks (Fosberg \& Rainey 60660 US)

Bidens comosa (A. Gray) Wiegand, Strawstem Beggar-ticks (Boone s.n. TAWES)

Bidens connata Muhl., Purplestem Beggar-ticks (Baltars 85 MARY)

Bidens discoidea (Torr. \& A. Gray) Britton, Swamp Beggar-ticks (Hotchkiss 7727 US)

Bidens frondosa L., Devil's Beggar-ticks (Blake 12094 US) 
Bidens laevis (L.) Britton, Sterns, \& Poggenb., Smooth Bur-marigold (Hotchkiss \& Ubler 7202 US)

[Bidens nashii Small]

- Bidens mitis (Michx.) Sherff, Small-Fruit Beggar-ticks-see Excluded Flora section.

- Bidens pilosa L., Hairy Beggar-ticks, † Waif (Reed 32882 US) [Bidens leucantha Willd.]

Bidens polylepis S. F. Blake, Ozark Tickseed Sunflower (Hermann 19351 US)

- Bidens tenuisecta A. Gray, Slim-Lobe Beggar-ticks, † Waif Reed (1964)

Bidens trichosperma (Michx.) Britton, Northern Tickseed Sunflower, S3S4/G5 (McCarthy s.n. TAWES)

[Bidens coronata (L.) Britton]

Bidens vulgata Greene, Tall Bur-marigold (Hitchcock 15717 US)

Boltonia asteroides (L.) L'Hér. var. asteroides, Susquehanna Doll's Daisy, S1/G5TNR (Long 75 PH)

Boltonia asteroides (L.) L'Hér. var. glastifolia (Hill) Fernald, White Doll's Daisy, S1/G5T4T5 (Knapp 3327 TAWES)

Borrichia frutescens (L.) DC., Sea Oxeye, SH/G5 (James s.n. MARY)

Brickellia eupatorioides (L.) Shinners var. eupatorioides, False Boneset (Steury 060930.1 US)

[Kubnia eupatorioides L.; K. glutinosa Elliott; K. kubnia (Gaertn.) C. Mohr.; K. mosieri Small]

Carduus acanthoides L., Spiny Plumeless Thistle, $\ddagger$ (Baltars 2500 US)

Carduus crispus L., Curled Plumeless Thistle, $\ddagger$ (Baltars 2729 US)

Carduus nutans L. ssp. macrolepis (Petermann) Kazmi, Musk Thistle, $\ddagger$ (Baltars 3101 US)

- Centaurea benedicta (L.) L., Blessed Thistle, † Waif Reed (1964)

[Carthamus tinctorius L.; Cnicus benedictus L.]

- Centaurea calcitrapa L., Big-head Purple Star-thistle, † Waif Reed (1964)

- Centaurea cineraria L., Dusty Miller-see Excluded Flora section.

Centaurea cyanus L., Garden Cornflower, † (Hermann 11495 US)

- Centaurea depressa M. M. Bieb., Iranian Knapweed, † Waif Reed (1964)

Centaurea jacea L., Brown Star-thistle, † (Baltars 747 US)

Centaurea nigra L., Black Star-thistle, † (Plitt 624 US)

Centaurea nigrescens Willd., Short-fringe Star-thistle, † (Norton s.n. US)

[Centaurea dubia Suter; C. vochinensis Bernh.]

- Centaurea scabiosa L., Great Star-thistle-see Excluded Flora section.

Centaurea solstitialis L., Yellow Star-thistle, † (Norton s.n. MARY)
Centaurea stoebe L. ssp. micranthos (S. G. Gmel. ex Gugler) Hayek, Spotted Star-thistle, † (Killip 32159 US) [Centaurea maculosa Lam.]

Chondrilla juncea L., Rush Skeletonweed, † (Crawford s.n. US)

Chrysogonum virginianum L. var. virginianum, Green and Gold, S3/G5T5 (Bartsch s.n. US)

[Chrysogonum australe Alexander ex Small]

Chrysopsis mariana (L.) Elliott, Maryland Goldenaster (Naczi 11574 DOV)

[Heterotheca mariana (L.) Shinners]

Cichorium intybus L., Chicory, † (Hotchkiss 5628 US)

Cirsium altissimum (L.) Spreng., Tall Thistle (Hitchcock s.n. US)

[Carduus altissimus L.]

Cirsium arvense (L.) Scop., Creeping Thistle, $\ddagger$ (Leonard 21590 US)

[Carduus arvensis (L.) Robson; C. arvense var. horridum Wimm. \& Grab]

Cirsium discolor (Muhl. ex Willd.) Spreng., Field Thistle (Longbottom $5353 \mathrm{DOV}$ )

[Carduus discolor (Muhl. ex Willd.) Nutt.]

Cirsium horridulum Michx. var. horridulum, Yellow Thistle, S3/G5 (Knapp 1209 TAWES)

[Carduus spinosissimus Walter; C. spinosissimus var. elliottii (Torr. \& A. Gray) Porter; Cirsium smallii Britton]

Cirsium muticum Michx., Swamp Thistle, S3/G5 (Baltars 529 US)

[Carduus muticus (Michx.) Pers.]

Cirsium pumilum Spreng., Pasture Thistle (Frye 5135 TAWES)

Cirsium vulgare (Savi) Ten., Bull Thistle, $\ddagger$ (Frye \& Lydon s.n. TAWES)

[Carduus lanceolatus L.]

Conoclinium coelestinum (L.) DC., Blue Boneset (Allard 3171 US) [Eupatorium coelestinum L.]

Conyza canadensis (L.) Cronquist var. canadensis, Canada Horseweed (Blake 8533 US)

[Erigeron canadensis L.; Leptilon canadense (L.) Britton]

Conyza canadensis (L.) Cronquist var. pusilla (Nutt.) Cronquist, Fleabane (Hill 15542 Assateague Island National Seashore Herbarium)

Coreopsis grandiflora Hogg ex Sweet, Large-flowered Tickseed, † (Leonard s.n. US)

[Coreopsis grandiflora var. harveyana Sherff; C. grandiflora var. saxicola (Alexander) E. B. Sm.; C. saxicola Alexander]

Coreopsis lanceolata L., Sand Coreopsis, † (King 7380 US)

[Coreopsis crassifolia Aiton] 
Coreopsis rosea Nutt., Rose Coreopsis, S1/G3 (Knapp 3327 TAWES)

Coreopsis tinctoria Nutt., Golden Tickseed, † (Blake 12091 US) [Coreopsis cardaminefolia (DC.) Torr. \& A. Gray]

Coreopsis tripteris L., Tall Tickseed, S1/G5 (Ward s.n. US)

Coreopsis verticillata L., Whorled Coreopsis, S3/G5 (Bartsch s.n. US)

- Cosmos parviflorus (Jacq.) Pers., Southwest Cosmos, † Waif (Reed 32916 US)

Cosmos sulphureus Cav., Sulphur Cosmos, † (Reed 32976 US)

- Cota tinctoria (L.) J. Gay, Golden Chamomile, † Waif (Norton s.n. US)

[Anthemis tinctoria L.]

-Crepis capillaris (L.) Wallr., Smooth Hawk's-beard, † Waif (Brummel s.n. US) [Labsana capillaris L.]

- Crepis pulchra L., Small-flower Hawk's-beard, † Waif (Baltars 3116 US)

- Crepis tectorum L., Narrowleaf Hawk's-beard, † Waif Brown and Brown (1984)

- Cymophora accedens (S. F. Blake) B. L. Turner \& A. M. Powel, Cymophora, † Waif (Reed 32899 US)

[Tridax accedens S. F. Blake]

Doellingeria infirma (Michx.) Greene, Appalachian Flat-topped White Aster, S3/G5 (Bartsch s.n. US)

[Aster infirmus Michx.; Doellingeria humilis (Willd.) Britton]

- Doellingeria sericocarpoides Small, Pocosin Flat-topped Aster - see Excluded Flora section.

Doellingeria umbellata (Mill.) Nees, Northern Flat-topped White Aster (Naczi 8955 DOV)

[Aster umbellatus Mill.]

Dyssodia papposa (Vent.) Hitchc., Fetid Dogweed, † (Cusick $38001 \mathrm{CM})$

[Tagetes papposa Vent.]

Echinacea pallida Nutt., Pale Purple Coneflower, † (King 6849 US)

[Brauneria pallida (Nutt.) Britton]

Echinacea purpurea (L.) Moench, Eastern Purple Coneflower, † (Baltars 3195 US)

[Brauneria purpurea (L.) Britton]

- Echinops sphaerocephalus L., Great Globe Thistle-see Excluded Flora section.

Eclipta prostrata (L.) L., False Daisy, † (Hotchkiss 5652 US) [Eclipta alba (L.) Hassk.; E. erecta L.]
Elephantopus carolinianus Willd., Carolina Elephant's-foot (Beyersdorfer 325 US)

Elephantopus nudatus A. Gray, Smooth Elephant's-foot (Naczi 11576 DOV)

Elephantopus tomentosus L., Tobaccoweed, S1S2/G5 (Steury 980803.8 US)

Erechtites hieraciifolius (L.) Raf. ex DC., American Burnweed (Smith 5156 US)

[Erechtites hieraciifolius var. intermedia Fernald; E. hieraciifolius var. praealta (Raf.) Fernald]

Erigeron annuus (L.) Pers., White-top Fleabane (Bartsch s.n. US)

Erigeron philadelphicus L., Philadelphia Fleabane (Knapp 2393 DOV)

Erigeron pulchellus Michx. var. brauniae Fernald, Lucy Braun's Plantain, S1/G5T4 (Knapp \& Thompson 3215 TAWES)

Erigeron pulchellus Michx. var. pulchellus, Robin's Plantain (Chase 6882 US)

Erigeron strigosus Muhl. ex Willd. var. strigosus, Daisy Fleabane (Hotchkiss 5201 US)

[Erigeron ramosus (Walter) Britton, Sterns, \& Poggenb.; E. strigosus var. beyrichii (Fisch. \& E. Mey.) A. Gray]

Eupatorium album L., White Thoroughwort (Engh s.n. MARY) [Eupatorium petaloideum Britton ex Small]

Eupatorium altissimum L., Tall Boneset, S3/G5 (Baltars 3048 US)

- Eupatorium cannabinum L., Hemp Agrimony, † Waif (Plitt s.n. US)

Eupatorium capillifolium (Lam.) Small, Small Dog-fennel Thoroughwort (Blake 11879 US)

Eupatorium godfreyanum Cronquist, Godfrey's Eupatorium (Steele s.n. US)

Eupatorium hyssopifolium L. var. hyssopifolium, Hyssopleaf Thoroughwort (Hotchkiss 5641 US)

[Eupatorium lecheifolium Greene]

Eupatorium leucolepis (DC.) Torr. \& A. Gray, White-bract Boneset, S2S3/G5 (Knapp \& Longbottom 1513 TAWES)

Eupatorium perfoliatum L., Common Boneset (Hotchkiss 5470 US)

Eupatorium pilosum Walter, Vervain Thoroughwort (Hotchkiss 5547 US)

[Eupatorium rotundifolium L. var. saundersii (Porter ex Britton) Cronquist]

Eupatorium rotundifolium L. var. ovatum (Bigelow) Torr., Hairy Boneset (Hill 16053 Assateague Island National Seashore Herbarium)

[Eupatorium pubescens Muhl. ex Willd.]

Eupatorium rotundifolium L. var. rotundifolium, Roundleaf Boneset (Hill 15470 Assateague Island National Seashore Herbarium)

[Eupatorium verbenifolium Michx.] 
- Eupatorium semiserratum DC., Small-flower Thoroughwortsee Excluded Flora section.

Eupatorium serotinum Michx., Late-flower Thoroughwort (Allard 3726 US)

Eupatorium sessilifolium L. var. sessilifolium, Upland Boneset (Killip 32663 US)

Eupatorium torreyanum Short \& R. Peter, Fringed Boneset (McAvoy 6717 DOV)

[Eupatorium hyssopifolium L. var. laciniatum A. Gray]

Eupatorium vaseyi Porter, Vasey's Boneset (Frye 960826-653 TAWES)

[Eupatorium album L. var. vaseyi (Porter) Cronquist; E. sessilifolium L. var. vaseyi (Porter) Fernald \& Griscom]

Eurybia compacta G. L. Nesom, Slender Aster (Benedict s.n. US) [Aster gracilis Nutt.]

Eurybia divaricata (L.) G. L. Nesom, White Wood Aster (Beyersdorfer 215 US)

[Aster divaricatus L.]

Eurybia macrophylla (L.) Cass., Largeleaf Wood Aster (Boone 830727 TAWES)

[Aster macrophyllus L.]

Eurybia radula (Aiton) G. L. Nesom, Rough Wood Aster, S1/G5 (Knapp 2976 TAWES)

[Aster radula Aiton]

Eurybia schreberi (Nees) Nees, Schreber's Aster (Morton 1990 US) [Aster schreberi Nees]

Eurybia spectabilis (Aiton) G. L. Nesom, Eastern Showy Aster, - S1/G5 (Knapp 174-03 TAWES)

[Aster spectabilis Aiton]

Euthamia caroliniana (L.) Greene ex Porter \& Britton, Carolina Goldentop (Naczi 11552 DOV)

[Euthamia microcephala Greene; E. tenuifolia (Pursh) Nutt.; Solidago tenuifolia Pursh]

Euthamia graminifolia (L.) Greene, Grassleaf Goldentop (Hitchcock 15730 US)

[Euthamia graminifolia var. nuttallii (Greene) W. Stone]

Euthamia gymnospermoides Greene, Texas Goldentop (Earle $2348 \mathrm{PH})$

Euthamia hirtipes (Fernald) Sieren, Marsh Goldentop (Earle $3015 \mathrm{PH})$

Eutrochium dubium (Willd. ex Poir.) E. E. Lamont, Joe-pye Thoroughwort (Hotchkiss 7599 US)

[Eupatoriadelphus dubius (Willd. ex Poir.) R. M. King \& H. Rob.; Eupatorium americanum Hill; E. dubium Willd. ex Poir.; E. ternifolium Elliott]

Eutrochium fistulosum (Barratt) E. E. Lamont, Hollow Joe-pyeweed (Hotchkiss 5419 US)

[Eupatoriadelphus fistulosus (Barratt) R. M. King \& H. Rob.; Eupatorium fistulosum Barratt]
Eutrochium maculatum (L.) E. E. Lamont var. maculatum, Spotted Joe-pyeweed, SU/G5 (Braun s.n. US)

[Eupatoriadelphus maculatus (L.) R. M. King \& H. Rob.; Eupatorium maculatum L.]

Eutrochium purpureum (L.) E. E. Lamont, Sweet Joe-pyeweed (Hitchcock 15842 US)

[Eupatoriadelphus purpureus (L.) R. M. King \& H. Rob.; Eupatorium amoenum Pursh; E. purpureum L.; E. trifoliatum L.]

Filago vulgaris Lam., Common Fluffweed, † (Eccles s.n. US)

- Flaveria trinervia (Spreng.) C. Mohr, Clustered Flaveria, † Waif (Reed 32997 US)

Gaillardia pulchella Foug. var. pulchella, Common Blanketflower, $†$ (Kalb s.n. MARY)

Galinsoga parviflora Cav., Small-flowered Quickweed, † (Killip 31943 US)

Galinsoga quadriradiata Ruiz \& Pav., Fringed Quickweed, † (Blake 8905 US)

[Galinsoga caracasana (DC.) Sch. Bip.; G. ciliata (Raf.) S. F. Blake]

Gamochaeta pensylvanica (Willd.) Cabrera, Pennsylvania Everlasting, † (Baltars 511 US)

Gamochaeta purpurea (L.) Cabrera, Purple Cudweed (Hotchkiss 5190 US)

[Gnaphalium purpureum L.]

- Glebionis segetum (L.) Fourr., Corn Marigold-see Excluded Flora section.

[Chrysanthemum segetum L.]

Gnaphalium uliginosum L., Low Cudweed, †? (Ditman s.n. MARY)

- Grindelia ciliata (Nutt.) Spreng., Spanish Gold, † Waif (Benedict s.n. US)

[Grindelia papposa G. L. Nesom \& Y. B. Suh; Haplopappus ciliatus (Nutt.) DC.; Prionopsis ciliatus (Nutt.) Nutt.]

Grindelia lanceloata Nutt., Narrowleaf Gumweed, † (Longbottom 21406 MARY)

Grindelia squarrosa (Pursh) Dunal, Broadleaf Gumweed, $\dagger$ (Brown s.n. MARY)

[Donia squarrosa Pursh]

- Guizotia abyssinica (L. f.) Cass., Ramtilla, † Waif Reed (1964)

Helenium amarum (Raf.) H. Rock var. amarum, Five-leaf Sneezeweed (Leonard \& Hotchkiss s.n. US)

[Helenium tenuifolium Nutt.] 
Helenium autumnale L. var. autumnale, Common Sneezeweed (Baltars 1662 US)

[Helenium autumnale var. pubescens (Aiton) Britton; $H$. latifolium Mill.; H. parviflorum Nutt.]

Helenium flexuosum Raf., Purple-head Sneezeweed (Hotchkiss 5497 US)

[Helenium nudiflorum Nutt.]

Helianthus angustifolius L., Swamp Sunflower (Naczi 11548 DOV)

[Helianthus angustifolius var. nemorosus C. Mohr]

Helianthus annuus L., Common Sunflower, † (Killip 32180 US)

Helianthus debilis Nutt. ssp. cucumerifolius (Torr. \& A. Gray) Heiser, Cucumberleaf Sunflower, † (Norton s.n. MARY) [Helianthus cucumerifolius Torr. \& A. Gray]

Helianthus decapetalus L., Thinleaf Sunflower (Beyersdorfer 140 US)

[Helianthus tracheliifolius Hook.]

Helianthus divaricatus L., Woodland Sunflower (Leonard \& Leonard 6216 US)

Helianthus giganteus L., Tall Sunflower (Hotchkiss 5535 US) [Helianthus alienus E. Watson]

Helianthus grosseserratus M. Martens, Sawtooth Sunflower, †? (Steury 02091.1 US)

Helianthus hirsutus Raf., Hairy Sunflower, S1/G5 (Shriver 257 MARY)

[Helianthus hirsutus var. stenophyllus Torr. \& A. Gray; H. hirsutus var. trachyphyllus Torr. \& A. Gray; H. stenophyllus (Torr. \& A. Gray) E. Watson]

Helianthus laevigatus Torr. \& A. Gray, Smooth Sunflower, - S1/G4 (Cusick 23880 MARY)

Helianthus maximiliani Schrad., Maximilian Sunflower, † (Harms 9693 DOV)

- Helianthus microcephalus Torr. \& A. Gray, Small Woodland Sunflower-see Excluded Flora section.

Helianthus mollis Lam., Ashy Sunflower, † (Leonard 6598 US)

Helianthus occidentalis Riddell ssp. occidentalis, Fewleaf Sunflower, S1/G5 (Blake 12090 US)

[Helianthus dowellianus M. A. Curtis]

- Helianthus pauciflorus Nutt., Stiff Sunflower, † Waif (Plitt 156 US)

- Helianthus salicifolius A. Dietr., Willowleaf Sunflower-see Excluded Flora section.

Helianthus strumosus L., Pale-leaf Sunflower (Leonard 1267 US) [Helianthus montanus E. Watson; H. saxicola Small]

Helianthus tuberosus L., Jerusalem Artichoke, † (Baltars 3250 US)

Heliopsis helianthoides (L.) Sweet var. helianthoides, Smooth Oxeye (Killip 32166 US)

- Helminthotheca echioides (L.) Holub, Bristly Oxtongue-see Excluded Flora section.

[Picris echioides L.]

- Heterosperma pinnatum Cav., Wingpetal, † Waif Reed (1964)
Heterotheca subaxillaris (Lam.) Britton \& Rusby, Camphorweed Goldenaster, † (Fosberg 30144 US)

[Heterotheca latifolia Buckley; H. subaxillaris var. latifolia (Buckley) Ghandi \& R. D. Thomas]

Hieracium aurantiacum L., Orange Hawkweed, † (Brighton Photo Voucher MPA)

Hieracium caespitosum Dumort., Meadow Hawkweed, † (Hermann s.n. US)

[Hieracium pretense Tausch; Pilosella caespitosa (Dumort.) P. D. Sell \& C. West]

Hieracium gronovii L., Hairy Hawkweed (Baltars 776 US)

Hieracium marianum Willd., Maryland Hawkweed (Owens s.n. MARY)

Hieracium paniculatum L., Panicled Hawkweed (Blake s.n. US)

Hieracium pilosella L., Mouse-ear Hawkweed, † (Cusick 23470 MARY)

[Pilosella officinarum F. Schultz \& Sch. Bip.]

Hieracium piloselloides Vill., Tall Hawkweed, † (Blake 11655 US)

[Pilosella piloselloides (Vill.) Soják]

Hieracium scabrum Michx., Rough Hawkweed (Dowell 6520 US)

Hieracium traillii Greene, Shale-barren Hawkweed (Stasz Photo Voucher MPA)

Hieracium venosum L., Rattlesnake Hawkweed (Foreman s.n. US)

Hypochaeris radicata L., Spotted Cat's-ear, † (Hermann 11615 US)

Inula helenium L., Elecampane, † (Baltars 2733 US)

Ionactis linariifolia (L.) Green, Flaxleaf Aster (Fosberg 16892 US)

[Aster linariifolius L.]

Iva annua L., Sumpweed (Longbottom 14356 DOV)

Iva frutescens L. ssp. frutescens, Bigleaf Marsh Elder (Earle $1527 \mathrm{PH})$

[Iva frutescens ssp. oraria (Bartlett) R. C. Jacks.]

- Krigia biflora (Walter) S. F. Blake var. biflora, Two-flowered Dwarf- Dandelion-see Excluded Flora section.

Krigia cespitosa (Raf.) K. L. Chambers, Opposite-Leaved Dwarf Dandelion, † (Harms 9556 US)

Krigia dandelion (L.) Nutt., Potato Dwarf Dandelion, S2S3/ G5 (Maxon \& Standley 328 US)

[Cynthia dandelion (L.) DC.]

Krigia virginica (L.) Willd., Virginia Dwarf Dandelion (Cowan 1693 US)

[Adopogon carolinianum (Walter) Britton]

Lactuca biennis (Moench) Fernald, Tall Blue Lettuce (Blake s.n. US)

Lactuca canadensis L., Canada Lettuce (Fosberg 19013 US) [Lactuca sagittifolia Elliott] 
Lactuca floridana (L.) Gaertn., Woodland Lettuce (Hotchkiss 5415 US)

[Lactuca floridana var. villosa (Jacq.) Cronquist; L. villosa Jacq.; Mulgedium floridanum (L.) DC.; M. villosum (Jacq.) Small]

Lactuca birsuta Muhl. ex Nutt., Hairy Lettuce, SH/G5? (Steele s.n. US)

Lactuca saligna L., Willowleaf Lettuce, † (Frye 6012 TAWES)

Lactuca serriola L., Prickly Lettuce, † (Hotchkiss 5988 US) [Lactuca scariola L.]

Lapsana communis L., Common Nipplewort, † (Steury 040611.1 US)

- Leontodon autumnalis L., Autumn Hawkbit, † Waif (Baltars 3178 US)

- Leontodon hispidus L., Common Hawkbit, † Waif (Arsène 20 US)

- Leontodon saxatilis Lam. ssp. saxatilis, Lesser Hawkbit, † Waif (Simmons et al. 1802 US)

[Leontodon leysseri (Wallr.) Beck]

Leucanthemum vulgare Lam., Oxeye Daisy, $†$ (Hotchkiss 5152 US)

[Chrysanthemum leucanthemum L.; Leucanthemum leucanthemum (L.) Rydb.]

Liatris belleri Porter, Heller's Blazing Star, SH/G3 (Baltars 4313 Cylburn Arboretum)

[Liatris turgida Gaiser]

Liatris pilosa (Aiton) Willd., Shaggy Gayfeather (Allard 3827 US) [Liatris graminifolia Willd.]

Liatris scariosa (L.) Willd., Devil's Bite (Knapp \& Thompson 3370 TAWES)

Liatris spicata (L.) Willd. var. spicata, Dense Blazing Star, - S1/G5T5 (Fleming 970823 TAWES)

[Lacinaria spicata (L.) Kuntze]

Liatris squarrosa (L.) Michx. var. squarrosa, Scaly Blazing Star, S1/G5 (Steury 826.1 US)

[Lacinaria squarrosa (L.) Hill]

Ligularia dentata (A. Gray) Hara, Orangequeen, † (Reese 7477 US)

- Madia sativa Molina, Chilean Tarweed, † Waif (Benedict 1043 US)

- Marshallia grandiflora Beadle \& F. E. Boynton, Appalachian Barbara's Buttons—-see Excluded Flora section.

- Matricaria chamomilla L., German Mayweed, † Waif (Godfrey 22 MARY)

[Matricaria recutita L.]

Matricaria discoidea DC., Pineapple-weed Chamomile, † (Smith 5284 US)

[Matricaria matricarioides (Less.) Porter]
- Melampodium sericeum Lag., Rough Blackfoot, † Waif (Reed 32739 US)

[Melampodium hispidum Kunth]

Mikania scandens (L.) Willd., Climbing Hempweed (Bartley 2362 US)

[Willoughbya scandens (L.) Kuntze]

Nabalus albus (L.) Hook., White Rattlesnake-root (Standley 5921 US)

[Prenanthes alba L.]

Nabalus altissimus (L.) Hook., Tall Rattlesnake-root (McAvoy 1323 DOV)

[Prenanthes altissima L.]

Nabalus autumnalis (Walter) Weakley, Slender Rattlesnakeroot, S1/G4G5 (Knapp 1069 TAWES)

[Prenanthes autumnalis Walter]

Nabalus crepidineus (Michx.) DC., Nodding Rattlesnake-root (Knapp 5025 MARY)

[Prenanthes crepidinea Michx.]

Nabalus serpentarius (Pursh) Hook., Lion's-foot (Naczi 8957 DOV)

[Prenanthes serpentaria Pursh]

Nabalus trifoliolatus Cass., Three-Leaved Rattlesnake-root (Longbottom 10501 DOV)

[Prenanthes trifoliolata (Cass.) Fernald]

Oclemena acuminata (Michx.) Greene, Whorled Aster (Hill 9551 MARY)

[Aster acuminatus Michx.]

Oclemena nemoralis (Aiton) Greene, Bog Aster, † (Wixted s.n. TAWES) - known at a single site in Anne Arundel County, where it appears to have been planted

[Aster nemoralis Aiton]

Oligoneuron rigidum (L.) Small var. rigidum, Prairie Goldenrod, - S1/G5 (Frye \& Knapp 6676 NCU)

[Oligoneuron grandiflorum (Raf.) Small; Solidago rigida L. var. rigida]

Onopordum acanthium L., Scotch Cotton-thistle, † (Baltars 732 US)

Packera anonyma (Alph. Wood) W. A. Weber \& Á. Löve, Small's Ragwort (Leonard 19863 US)

[Senecio anonymus Alph. Wood; S. aureus L. var. angustifolius Britton; S. earlei Small; S. smallii Britton]

Packera antennariifolia (Britton) W. A. Weber \& Á. Löve, Cat's-paw Ragwort, S3/G4 (Frye \& Frye 5912 TAWES) [Senecio antennariifolius Britton]

Packera aurea (L.) Á. Löve \& D. Löve, Golden Groundsel (Beyersdorfer 41 US)

[Senecio aureus L.] 
Packera crawfordii (Britton) A. M. Mahoney \& R. Kowa, Crawford's Ragwort (Maxon \& Standley $377 \mathrm{MO}$ ) [Senecio crawfordii Britton]

- Packera glabella (Poir.) C. Jeffrey, Butterweed-see Excluded Flora section. [Senecio glabellus Poir.]

Packera obovata (Willd.) W. A. Weber \& Á. Löve, Roundleaf Groundsel (Blake 6863 US)

[Senecio obovatus Willd.]

Packera paupercula (Michx.) Á. Löve \& D. Löve, Balsam Ragwort, S3/G5 (Maxon 6304 US)

[Senecio pauperculus Michx.]

Packera tomentosa (Michx.) C. Jeffrey, Woolly Groundsel (Freeman s.n. US)

[Senecio tomentosus Michx.]

- Parthenium auriculatum Britton, Glade Wild Quinine-see Excluded Flora section.

[P. hispidum Raf. var. auriculatum (Britton) Rollins]

- Parthenium hysterophorus L., Santa Maria Feverfew, † Waif Reed (1964)

Parthenium integrifolium L., American Feverfew, S1/G5 (Standley s.n. US)

- Picris hieracioides L., Hawkweed Oxtongue, † Waif (Norton s.n. MARY)

Pityopsis graminifolia (Michx.) Nutt. var. latifolia (Fernald) Semple \& F. D. Bowers, Narrowleaf Silk-grass (Naczi 11561 DOV)

[Chrysopsis graminifolia (Michx.) Nutt. var. latifolia Fernald; Heterotheca nervosa (Willd.) Shinners var. nervosa]

Pluchea camphorata (L.) DC., Marsh Fleabane, S1/G5 (Knapp 2631 TAWES)

[Pluchea petiolata Cass.]

Pluchea foetida (L.) DC. var. foetida, Stinking Camphorweed (McAvoy 4752 DOV)

[Pluchea tenuifolia Small]

Pluchea odorata (L.) Cass. var. odorata, Scented Conyza (Bartsch s.n. US)

[Pluchea odorata var. succulenta (Fernald) Cronquist; P. purpurascens (Sw.) DC.; P. purpurascens var. succulenta Fernald]

Polymnia canadensis L., White-flowered Leafcup (Beyersdorfer 441 US)

[Polymnia canadensis var. radiata A. Gray; $P$. radiata (A. Gray) Small]

Pseudognaphalium helleri (Britton) Anderb., Catfoot (Blake 8498 US)

[Gnaphalium helleri Britton; G. obtusifolium var. helleri (Britton) S. F. Blake]
Pseudognaphalium obtusifolium (L.) Hilliard \& B. L. Burtt, Fragrant Cudweed (Knapp 2592 DOV)

[Gnaphalium obtusifolium L.]

Pulicaria dysenterica (L.) Bernh., Meadow False Fleabane, † (Freeman s.n. US)

Pyrrhopappus carolinianus (Walter) DC., Carolina False Dandelion (Leonard 21900 US)

[Sitilias caroliniana (Walter) Raf.]

Rudbeckia fulgida Aiton var. fulgida, Orange Coneflower, - S3/G5 (Harger 14787 US)

[Rudbeckia acuminata F. E. Boynton \& Beadle; R. foliosa

F. E. Boynton \& Beadle; R. spathulata Michx.; R. tenax F. E. Boynton \& Beadle; R. truncata Small]

Rudbeckia hirta L. var. hirta, Black-eyed Susan (Reveal 5459 MARY)

[Rudbeckia amplectens T. V. Moore; R. brittonii Small; R. monticola Small]

Rudbeckia hirta L. var. pulcherrima Farw., Showy Black-eyed Susan, †? (Hamilton 525 Assateague Island National Seashore Herbarium)

[Rudbeckia longipes T. V. Moore; R. sericea T. V. Moore; R. serotina Nutt.]

Rudbeckia laciniata L. var. bipinnata Perdue, Greenhead Coneflower (Downs 4365 NCU)

Rudbeckia laciniata L. var. bumilis A. Gray, Blue Ridge Cutleaf Coneflower (Windler et al. $3257 \mathrm{NCU}$ )

[Rudbeckia digitata Mill.; R. laciniata var. digitata (Mill.) Fiori]

Rudbeckia laciniata L. var. laciniata, Common Greenhead Coneflower (Longbottom 3363 MARY)

Rudbeckia triloba L. var. triloba, Brown-eyed Susan, S3/G5 (Crawford s.n. US)

- Sanvitalia procumbens Lam., Spreading Sanvitalia, † Waif (Reed 32783 US)

[Sanvitalia angustifolia Engelm.]

- Schkubria pinnata (Lam.) Kuntze ex Thell, Pinnate False Threadleaf, † Waif Reed (1964)

[Schkuhria wislizenii A. Gray]

Sclerolepis uniflora Britton, Sterns, \& Poggenb., One-flowered Sclerolepis, S2/G4 (Knapp 566 DOV)

- Senecio bicolor (Willd.) Viv., Silver Ragwort-see Excluded Flora section.

[Senecio cineraria DC.]

Senecio suaveolens (L.) Elliott, Sweet-scented Indian-plantain, S1/G4 (Thompson o Berdine s.n. TAWES)

[Cacalia suaveolens L.; Hasteola suaveolens (L.) Pojark; Synosma suaveolens (L.) Raf.]

Senecio vulgaris L., Old-man-in-the-spring, † (Hyacinth 1752 US) 
Sericocarpus asteroides (L.) Britton, Sterns, \& Poggenb., Toothed Whitetop Aster (Hotchkiss 5331 US)

[Aster paternus Cronquist]

Sericocarpus linifolius (L.) Britton, Sterns, \& Poggenb., Narrowleaf Whitetop Aster (Hotchkiss 5333 US)

[Aster solidagineus Michx.]

Silphium asteriscus L. var. trifoliatum (L.) Clevinger, Threeleaf Rosinweed, S3/G4? (Killip 36697 US)

[Silphium trifoliatum L.]

- Silphium perfoliatum L. var. connatum Cronquist, Virginia Cup Plant-see Excluded Flora section.

- Silphium perfoliatum L. var. perfoliatum, Cup Plant-see Excluded Flora section.

Silybum marianum (L.) Gaertn., Blessed Milk-thistle, † (Norton s.n. MARY)

[Mariana mariana (L.) Hill]

- Simsia lagascaeformis DC., Annual Bush Sunflower, † Waif (Reed 32985 US)

Smallanthus uvedalia (L.) Mack., Bear's-foot (Knapp 2945 DOV)

[Polymnia uvedalia (L.) L.]

Solidago altissima L., Tall Goldenrod (Boone 830903 TAWES) [Solidago canadensis var. scabra Torr. \& A. Gray; S. canadensis var. scabriuscula Porter; S. hirsutissima Mill.]

Solidago arguta Aiton var. arguta, Cutleaf Goldenrod, S1? G5T4T5 (Baltars 1672 MARY)

- Solidago arguta Aiton var. boottii (Hook.) E. J. Palmer \& Steyerm., Boot's Goldenrod-see Excluded Flora section.

Solidago arguta Aiton var. caroliniana A. Gray, Atlantic Goldenrod, S1?/G5T4 (Frye 6278 TAWES)

Solidago bicolor L., White Goldenrod (Boone s.n. TAWES)

Solidago caesia L., Bluestem Goldenrod (Bodkin 157 MARY)

Solidago canadensis L., Canada Goldenrod (Hill 15544 Assateague Island National Seashore Herbarium)

[Solidago canadensis L. var. hargeri Fernald]

- Solidago curtisii Torr. \& A. Gray, Curtis' Goldenrod-see Excluded Flora section.

[Solidago lancifolia Torr. \& A. Gray; S. pubens M. A. Curtis]

Solidago erecta Pursh, Slender Goldenrod (Knapp 2596 TAWES)

Solidago fistulosa Mill., Pine Barren Goldenrod (Knapp 2591 TAWES)

Solidago flexicaulis L., Broadleaf Goldenrod (Longbottom 2746 MARY)

Solidago gigantea Aiton, Smooth Goldenrod (Norton s.n. US) [Solidago serotina Aiton var. gigantea (Aiton) A. Gray; S. serotina Aiton]

Solidago harrisii E. S. Steele, Shale Barren Goldenrod, S3/G4 (Frye \& Knapp 6677 TAWES)

[Solidago arguta Aiton var. harrisii (E. S. Steele) Crounquist]
Solidago hispida Muhl. ex Willd., Hairy Goldenrod, S3/G5T5 (Frye 6276 TAWES)

Solidago juncea Aiton, Early Goldenrod (Baltars 4169 US)

Solidago latissimifolia Mill., Elliott's Goldenrod, S3/G5 (Steele s.n. US)

[Solidago edisoniana Mack.; S. elliottii Torr. \& A. Gray; S. mirabilis Small]

- Solidago missouriensis Nutt., Missouri Goldenrod-see Excluded Flora section.

Solidago nemoralis Aiton ssp. nemoralis, Gray Goldenrod (Frye 6270 TAWES)

Solidago odora Aiton var. odora, Anise-scented Goldenrod (Leonard 915 US)

[Solidago odora var. inodora Gray]

Solidago patula Muhl. ex Willd. var. patula, Roundleaf Goldenrod, S3/G5 (Fessenden \& Baltars 1898 US)

Solidago puberula Nutt. var. puberula, Downy Goldenrod (Knapp 2602 DOV)

- Solidago puberula Nutt. var. pulverulenta (Nutt.) Chapm., Powdery Goldenrod-see Excluded Flora section.

[Solidago pulverulenta Nutt.]

Solidago racemosa Greene, Racemose Goldenrod, S1/G5T3? (Bartsch s.n. US)

[Solidago simplex Kunth var. racemosa (Greene) G. S. Ringius; S. spathulata DC.]

Solidago roanensis Porter, Roan Mountain Goldenrod, - S3/G4G5 (Frye 6693 TAWES)

Solidago rugosa Mill. var. aspera (Aiton) Fernald, Roughleaf Goldenrod (Hill 15682 Assateague Island National Seashore Herbarium)

[Solidago celtidifolia Small; S. rugosa ssp. aspera (Aiton) Cronquist; S. rugosa var. celtidifolia (Small) Fernald]

Solidago rugosa Mill. var. rugosa, Roughleaf Goldenrod (Terrell 6213 MARY)

[Solidago rugosa ssp. villosa (Pursh) Cronquist]

- Solidago rugosa Mill. var. sphagnophila C. Graves, Peat-loving Goldenrod-see Excluded Flora section.

Solidago rupestris Raf., Rock Goldenrod, S1/G4? (Knapp 5299 WAT)

Solidago sempervirens L. var. mexicana (L.) Fernald, Southern Seaside Goldenrod (Hill 15858 Assateague Island National Seashore Herbarium)

[Solidago angustifolia Elliott; S. mexicana L.; S. petiolata Mill.]

Solidago sempervirens L. var. sempervirens, Seaside Goldenrod (Hill 15837 Assateague Island National Seashore Herbarium)

Solidago speciosa Nutt. var. speciosa, Harper's Goldenrod, S2/G5 (McAvoy 1959 DOV)

[Solidago conferta Mill.; S. harperi Mack.]

Solidago squarrosa Nutt., Ragged Goldenrod (Frye 6279 TAWES)

- Solidago stricta Aiton, Pine Barren Bog Goldenrod-see Excluded Flora section. 
Solidago tarda Mack., Late Goldenrod, S1/G4? (Frye 6406 TAWES)

Solidago uliginosa Nutt., Bog Goldenrod, S3/G5 (Leonard 919 US)

[Solidago neglecta Torr. \& A. Gray; S. uniligulata (DC.) Porter]

Solidago ulmifolia Muhl. var. ulmifolia, Elmleaf Goldenrod, - S3/G4G5 (Hitchcock 15735 US)

Sonchus arvensis L., Field Sowthistle, † (Fessenden s.n. US) [Sonchus arvensis var. glabrescens Geunth., Grab. \& Wimm.; S. uliginosus M. Bieb.]

Sonchus asper (L.) Hill, Spiny-leaf Sowthistle, † (Leonard 20879 US)

Sonchus oleraceus L., Common Sowthistle, † (King \& Krantz 40 US)

Symphyotrichum concolor (L.) G. L. Nesom, Eastern Silvery Aster, S1/G5 (Hotchkiss 7290 US)

[Aster concolor L.; A. plumosus Small]

Symphyotrichum cordifolium (L.) G. L. Nesom, Heartleaf Aster (Frye 6290 TAWES)

[Aster cordifolius L.]

Symphyotrichum depauperatum (Fernald) G. L. Nesom, Serpentine Aster, S1/G2 (McAvoy 702 DOV) [Aster depauperatus Fernald]

Symphyotrichum drummondii (Lindl.) G. L. Nesom, Drummond's Aster, S1/G5 (Lea 913 TAWES)

[Aster drummondii Lindl.]

Symphyotrichum dumosum (L.) G. L. Nesom, Bushy Aster (Hotchkiss 5554 US)

[Aster dumosus L.; A. dumosus var. coridifolius (Michx.) Torr. \& A. Gray]

Symphyotrichum ericoides (L.) G. L. Nesom, White Heath Aster (Davis 3946 US)

[Aster ericoides L.; A. multiflorus Aiton]

Symphyotrichum laeve (L.) Á. Löve \& D. Löve var. concinnum (Willd.) G. L. Nesom, Narrow-leaved Smooth Blue Aster, - S1?/G5T4 (Boone s.n. TAWES)

[Aster concinnus Willd.; A. laevis var. concinnus (Willd.) House]

Symphyotrichum laeve (L.) Á. Löve \& D. Löve var. laeve, Smooth Blue Aster (Boone s.n. TAWES)

[Aster falcidens E. S. Burgess; A. laevis L.; A. laevis var. amplifoius Porter; A. steeleorum Shinners]

Symphyotrichum lanceolatum (Willd.) G. L. Nesom var. lanceolatum, Panicled Aster (Blake s.n. US)

[Aster lanceolatus Willd.; A. simplex Willd. var. ramosissimus (Torr. \& A. Gray) Cronquist]

Symphyotrichum lanceolatum (Willd.) G. L. Nesom var. latifolium (Semple \& Chmiel.) G. L. Nesom, White Panicled Aster (Hill \& Klein 15956 MARY)

[Aster lanceolatus Willd. var. latifolium Semple \& Chmiel.]
Symphyotrichum lateriflorum (L.) Á. Löve \& D. Löve, Starved Aster (Baltars 3252 US)

[Aster hirsuticaulis Lindl. ex DC.; S. lateriflorus (L.) Britton; Symphyotrichum lateriflorum var. horizontale (Desf.) G. L. Nesom]

Symphyotrichum lowrieanum (Porter) G. L. Nesom, Fall Aster (Lea 937 TAWES)

[Aster lowrieanum Porter]

Symphyotrichum novae-angliae (L.) G. L. Nesom, New England Aster (Kearney 215 US)

[Aster novae-angliae L.]

Symphyotrichum novi-belgii (L.) G. L. Nesom, Longleaf Aster (Maxon \& Standley 41 US)

[Aster novi-belgii L.; A. novi-belgii var. elodes (Torr. \& A. Gray) A. Gray]

Symphyotrichum oblongifolium (Nutt.) G. L. Nesom, Aromatic Aster (Pollard 765 US) [Aster oblongifolius Nutt.]

Symphyotrichum patens (Aiton) G. L. Nesom, Late Purple Aster (Kearney Jr. 208 US)

[Aster patens Aiton; A. patens var. gracilis Hook.; A. patens var. tenuicaulis C. Mohr; A. tenuicaulis (C. Mohr) E. S. Burgess]

Symphyotrichum phlogifolium (Muhl. ex Willd.) G. L. Nesom, Late Purple Aster (Stasz Photo Voucher MPA) [Aster phlogifolius Muhl. ex Willd.]

Symphyotrichum pilosum (Willd.) G. L. Nesom var. pilosum, White Heath Aster (Harms 7109 MARY) [Aster pilosus Willd.]

Symphyotrichum pilosum (Willd.) G. L. Nesom var. pringlei (A. Gray) G. L. Nesom, Pringle's Aster (Lea 897 TAWES) [Aster pilosus Willd. var. pringlei A. Gray]

Symphyotrichum praealtum (Poir.) G. L. Nesom, Willow Aster, S1/G5 (Hill 9504 MARY)

[Aster praealtus Poir.]

Symphyotrichum prenanthoides (Muhl. ex Willd.) G. L. Nesom, Crooked-stem Aster (Kearney Jr. 232 US)

[Aster prenanthoides Muhl. ex Willd.]

Symphyotrichum puniceum (L.) Á. Löve \& D. Löve, Swamp Aster (Hotchkiss 5556 US) [Aster puniceus L. var. scabricaulis (Shinners) A. G. Jones]

Symphyotrichum racemosum (Elliott) G. L. Nesom, Small White Aster (Bartsch s.n. US) [Aster racemosus Elliott]

Symphyotrichum shortii (Lindl.) G. L. Nesom, Short's Aster, - S3S4/G5 (Palmer s.n. US)

[Aster camptosorus Small; A. shortii Lindl.]

Symphyotrichum subulatum (Michx.) G. L. Nesom, Annual Saltmarsh Aster (Cluley \& Leonard 20970 US) [Aster subulatus Michx.]

Symphyotrichum tenuifolium (L.) G. L. Nesom, Perennial Saltmarsh Aster (Hotchkiss 7287 US)

[Aster tenuifolius L.; A. vimineus Lam. var. foliolosus (Aiton) A. Gray] 
Symphyotrichum undulatum (L.) G. L. Nesom, Wavyleaf Aster (Pollard 695 US)

[Aster asperifolius E. S. Burgess; A. claviger E. S. Burgess; A. corrigiatus E. S. Burgess; A. loriformis E. S. Burgess; A. mohrii E. S. Burgess; A. proteus E. S. Burgess; A. sylvestris E. S. Burgess; A. triangularis E. S. Burgess; A. truellius E. S. Burgess; A. undulatus L.; A. undulatus var. diversifolius (Michx.) A. Gray; A. undulatus var. loriformis E. S. Burgess]

Symphyotrichum urophyllum (Lindl.) G. L. Nesom, White Arrow Aster (Lea 911 TAWES)

[Aster sagittifolius Wedem. ex Willd.; A. urophyllus Lindl.]

- Tagetes erecta L., Aztec Marigold, † Waif Reed (1964)

- Tagetes minuta L., Muster John Henry, † Waif (Reed 32890 US)

- Tagetes pusilla L., Lesser Marigold, † Waif Reed (1964)

Tanacetum balsamita L., Coastmary, † (Brown s.n. MARY) [Chrysanthemum balsamita (L.) Baill.]

Tanacetum parthenium (L.) Sch. Bip., Common Feverfew, † (Leonard 20911 US)

[Chrysanthemum parthenium (L.) Bernh.]

Tanacetum vulgare L., Common Tansy, † (Longbottom 11777 US)

Taraxacum erythrospermum Andrz. ex Besser, Red-seeded Dandelion, † (Hitchcock 12476 US)

[Leontodon erythrospermum (Andrz. ex Besser) Britton; Taraxacum laevigatum (Willd.) DC.]

Taraxacum officinale Wiggers ssp. officinale, Common Dandelion, $†$ (Beyersdorfer 55 US)

[Leontodon taraxacum L.; Taraxacum taraxacum (L.) H. Karst.]

Tragopogon dubius Scop., Meadow Goat's-beard, † (Baltars 2967 US)

[Tragopogon major Jacq.]

Tragopogon porrifolius L., Purple Goat's-beard, † (Hill 10376 MARY)

Tragopogon pratensis L., Meadow Goat's-beard, † (Longbottom et al. $8776 \mathrm{DOV})$

- Tripleurospermum inodorum (L.) Sch. Bip., Scentless Chamomile-see Excluded Flora section.

[Matricaria inodora L.; Tripleurospermum perforata (Mérat) M. Lainz]

Tripleurospermum maritimum (L.) W. D. J. Koch ssp. maritimum, Pineapple Weed, † (Ditman s.n. MARY)

[Matricaria maritima L.]

Tussilago farfara L., Colt's-foot, $†$ (Baltars 588 US)

Verbesina alternifolia (L.) Britton ex Kearney, Wingstem (Hitchcock 15710 US)

[Ridan alternifolia (L.) Britton]
- Verbesina encelioides (Cav.) Benth. \& Hook. ex A. Gray, Cowpen Crownbeard, † Waif Reed (1964)

[Verbesina encelioides var. exauriculata B. L. Rob. \& Greenm.; Ximenesia encelioides Cav.]

Verbesina occidentalis (L.) Walter, Yellow Crownbeard (Allard 37239 US)

[Phaethusa occidentalis (L.) Britton]

- Verbesina virginica L., White Crownbeard-see Excluded Flora section.

Vernonia arkansana DC., Arkansas Ironweed, † (Harms 6915 MARY)

Vernonia gigantea (Walter) Trel., Giant Ironweed, SU/G5 (McDowell Photo Voucher MPA)

[Vernonia altissima Nutt.; V. gigantea ssp. ovalifolia (Torr. \& A. Gray) Urbatsch; V. maxima Small; V. ovalifolia Torr. $\&$ A. Gray]

Vernonia glauca (L.) Willd., Broadleaf Ironweed (Kearney Jr. 143 US)

Vernonia noveboracensis (L.) Michx., New York Ironweed (Longbottom 5354 DOV)

[Vernonia harperi Gleason]

Xanthium spinosum L., Spiny Cocklebur, † (Smith s.n. US) [Acanthoxanthium spinosum (L.) Fourr.]

Xanthium strumarium L., Rough Cocklebur, $\ddagger$ (Baltars 405 US) [Xanthium americanum Walter; X. canadense Mill.; X. chinense Mill.; X. cylindraceum Millsp. \& Sherff; X. echinatum Murray; X. echinellum Greene; X. pensylvanicum Wallr.; X. speciosum Kearney; X. strumarium var. canadense (Mill.) Torr. \& A. Gray; X. strumarium var. glabratum (DC.) Cronquist]

Youngia japonica (L.) DC., Oriental False Hawksbeard, $\ddagger$ (Baltars 3088 US)

[Crepis japonica (L.) Benth.]

Youngia thunbergiana DC., False Hawksbeard, † (Hill 16755 MO)

BALSAMINACEAE (Touch-me-not Family)

Impatiens balsamina L., Spotted Snapweed, † (McAtee 2854 US)

Impatiens capensis Meerb., Orange Jewelweed (Tidestrom 7441 DOV) [Impatiens biflora Walter]

Impatiens pallida Nutt., Pale Jewelweed (Longbottom 4315 DOV)

[Impatiens aurea Muhl.]

BERBERIDACEAE

(Barberry Family)

- Berberis canadensis Mill., American Barberry-see Excluded Flora section.

Berberis julianae C. K. Schneid., Wintergreen Barberry, † (Harms 9481 US) 
Berberis thunbergii DC., Japanese Barberry, $\ddagger$ (Seyfried \& Dill R85-64 DOV)

Berberis vulgaris L., European Barberry, † (Grant 89-00456 DOV)

Caulophyllum giganteum (Farw.) Loconte \& W. H. Blackw., Giant Blue Cohosh (Eifrick s.n. CM)

Caulophyllum thalictroides (L.) Michx., Blue Cohosh (Terrell 4086 US)

Jeffersonia diphylla (L.) Pers., Twinleaf (Knapp 2402b TAWES)

Mahonia bealei (Fortune) Carrière, Beale's Barberry, † (Grant 89-00551 DOV)

[Berberis bealei Fortune]

Nandina domestica Thunb., Sacred Bamboo, † (Byrne 9-08D BALT)

Podophyllum peltatum L., Mayapple (Driskill et al. CEP851297 DOV)

\section{BETULACEAE}

(Birch Family)

Alnus glutinosa (L.) Gaertn., Black Alder, † (Lea 2458 US)

Alnus incana (L.) Moench var. rugosa (Du Roi) R. T. Clausen, Speckled Alder (Norton 518 MARY)

[Alnus rugosa (Du Roi) Spreng.]

Alnus maritima (Marshall) Muhl. ex Nutt. ssp. maritima, Seaside Alder, S3.1/G3 (Knapp 2641 DOV)

Alnus serrulata (Aiton) Willd., Common Alder (Knapp 2640 DOV)

Betula alleghaniensis Britton, Yellow Birch (Hermann 13751 US) [Betula lutea Michx.]

Betula lenta L., Sweet Birch (Dill 120 DOV)

Betula nigra L., River Birch (Dill 577 DOV)

- Betula papyrifera Marshall, Paper Birch-see Excluded Flora section.

Betula populifolia Marshall, Gray Birch, S1?/G5 (Baltars 1425 US)

- Betula pubescens Ehrh., Downy Birch, † Waif (Clark 17 MARY)

[Betula alba L.]

Carpinus caroliniana Walter, Ironwood (Hotchkiss 5170 US) [Carpinus caroliniana ssp. virginiana (Marshall) Furlow; C. caroliniana var. virginiana (Marshall) Fernald]

Corylus americana Walter, American Hazelnut (Uhler s.n. US)

Corylus cornuta Marshall var. cornuta, Beaked Hazelnut, S3/ G5 (Norton s.n. MARY)

[Corylus rostrata Aiton]

Ostrya virginiana (Mill.) K. Koch, Eastern Hop-hornbeam (Naczi 9328a DOV)
BIGNONIACEAE (Trumpet Creeper/Bignonia Family)

Bignonia capreolata L., Crossvine (McAvoy 3690 DOV) [Anisostichus capreolata (L.) Bureau; A. crucigera (L.) Bureau]

Campsis radicans (L.) Bureau, Trumpet Creeper (Driskill et al. CEP 85-1370 DOV)

[Bignonia radicans L.]

Catalpa bignonioides Walter, Southern Catalpa, † (Killip 36837A US)

[Catalpa catalpa (L.) Karsten]

- Catalpa ovata G. Don, Chinese Catalpa, † Waif-Brown and Brown (1972:295) stated, "Reported in Gray's Manual as an escape from cultivation in MD." If this does escape, it is not an established component of the flora. Olsen and Kirkbride (2017) cited a Reed collection from Frederick County that may be a noncultivated plant.

Catalpa speciosa (Warder) Engelm., Northern Catalpa, † (Hotchkiss 6999 US)

BORAGINACEAE

(Borage Family)

Anchusa arvensis (L.) M. Bieb., Small Bugloss, † (Foreman s.n. US)

[Lycopsis arvensis L.]

- Anchusa azurea Mill., Italian Bugloss, † Waif Reed (1964) [Anchusa italica Retz.]

Andersonglossum virginianum (L.) J. I. Cohen, Wild Comfrey (Knapp 2333 DOV)

[Cynoglossum virginianum L.]

- Asperugo procumbens L., Madwort-see Excluded Flora section.

- Borago officinalis L., Common Borage-see Excluded Flora section.

Brunnera macrophylla (Adams) I. M. Johnst., Largeleaf Brunnera, † (Hall JH2015.05.04-1 MARY)

Buglossoides arvensis (L.) I. M. Johnst., Corn Gromwell, † (Longbottom 12472 DOV)

[Lithospermum arvense L.]

Cynoglossum officinale L., Common Hound's-tongue, † (Braendle s.n. US)

- Echium italicum L., Italian Viper's Bugloss, † Waif Reed (1964)

Echium vulgare L., Common Viper's Bugloss, † (Otis s.n. DOV)

Ellisia nyctelea (L.) L., Nyctelea (Ubler \& Erickson s.n. US)

Hackelia virginiana (L.) I. M. Johnst., Virginia Stickseed (McAvoy 5173 DOV)

[Lappula virginiana (L.) Greene] 
- Heliotropium curassavicum L., Seaside Heliotrope, † Waif Reed (1964)

- Heliotropium europaeum L., European Heliotrope, † Waif (Tatnall 4576 DOV)

- Heliotropium indicum L., Indian Heliotrope, † Waif Reed (1964)

[Tiaridium indicum (L.) Lehm.]

- Heliotropium procumbens Mill., Four-spike Heliotrope, † Waif Reed (1964)

Hydrophyllum canadense L., Bluntleaf Waterleaf (Constance 3024 US)

Hydrophyllum macrophyllum Nutt., Largeleaf Waterleaf, S2/G5 (Boone s.n. TAWES)

Hydrophyllum virginianum L., John's-cabbage (Knapp 2401 DOV)

Lappula squarrosa Dumort., Bristly Stickseed, † (Arsène s.n. US) [Lappula echinata Gilbert ex Fritsch]

Lithospermum canescens (Michx.) Lehm., Hoary Puccoon (Boone s.n. TAWES)

[Batschia canescens Michx.]

Lithospermum latifolium Michx., American Gromwell, S1/G4 (Leonard 2668 US)

- Lithospermum molle (Michx.) Muhl., Soft-hair Marbelseedsee Excluded Flora section.

[Onosmodium molle Michx. var. hispidissimum (Mack.) Cronquist]

Lithospermum parviflorum Weakley, Witsell, \& D. Estes, Softhair False Gromwell, S1/G4G5 (Boone s.n. TAWES) [Onosmodium hispidissimum Mack.]

Lithospermum virginianum L., Virginia False Gromwell, - S1/G4 (Canby s.n. DOV)

[Onosmodium virginianum (L.) A. DC.]

Mertensia virginica (L.) Pers. ex Link, Virginia Bluebells (Fosberg 16966 US)

Myosotis arvensis (L.) Hill, Rough Forget-me-not, † (Wootten s.n. DOV)

Myosotis discolor Pers., Yellow-and-blue Forget-m -not, † (Hill 11118 MARY)

[Myosotis versicolor $\mathrm{Sm}$.

Myosotis laxa Lehm., Small Forget-me-not (Otis s.n. DOV)

Myosotis macrosperma Engelm., Large-seeded Forget-me-not, - S3S4/G5 (Steury 980523.2 US)

[Myosotis verna Nutt. var. macrosperma (Engelm.) Chapm.]

Myosotis scorpioides Lam., True Forget-me-not, † (Terrell 4257 US)

Myosotis stricta Link ex Roem. \& Schult., Small-Flowered Forget-Me-Not, † (Longbottom 5692 DOV)

Myosotis verna Nutt., Spring Forget-me-not, S3/G5 (Steury 990612.5 US)

[Myosotis virginica (L.) Britton, Sterns, \& Poggenb.]
- Nama hispida A. Gray, Rough Fiddleleaf, † Waif Reed (1964) [Marilaunidium hispidum (A. Gray) Kuntze]

Nemophila aphylla (L.) Brummitt, Small-flowered Baby Blueeyes, S2/G5 (Steury 990428 US)

[Nemophila microcalyx (Nutt.) Fischer \& C. A. Meyer]

- Phacelia bipinnatifida Michx., Fernleaf Phacelia-see Excluded Flora section. [Phacelia brevistyla Buckley]

Phacelia covillei S. Watson ex A. Gray, Buttercup Scorpionweed, - S2/G3 (Terrell 4082 US)

Phacelia dubia (L.) Trel., Small-flowered Scorpionweed (Shetler 2004.4 US)

Phacelia purshii Buckley, Miami Mist, S3/G5 (Uhler s.n. US) [Phacelia bicknellii Small; P. boykinii (Gray) Small]

- Phacelia ranunculacea (Nutt.) Constance, Oceanblue Phacelia-see Excluded Flora section.

- Symphytum asperum Lepech., Prickly Comfrey—see Excluded Flora section.

Symphytum officinale L., Common Comfrey, † (Olds s.n. US)

BRASSICACEAE [Cruciferae] (Mustard Family)

Alliaria petiolata (M. Bieb.) Cavara \& Grande, Garlic Mustard, $\ddagger($ Knapp 2388 DOV)

[Alliaria officinalis Andrz.]

Alyssum alyssoides (L.) L., Pale Alyssum, † (Rowan et al. 102 MARY)

Arabidopsis lyrata (L.) O'Kane \& Al-Shehbaz ssp. lyrata, Lyreleaf Rockcress (Ebert \& Holt 105EH DOV) [Arabis lyrata L.]

Arabidopsis thaliana (L.) Heynh., Wall-cress, † (McAvoy 2118 DOV)

[Stenophragma thalianum (L.) Čelak.]

Arabis patens Sull., Spreading Rockcress, S3/G3 (Mearns s.n. US)

Arabis pycnocarpa M. Hopkins var. adpressipilis M. Hopkins, Slender Rockcress, S1S2/G5T4Q (Beckman s.n. US) [Arabis hirsuta (L.) Scop. var. adpressipilis (M. Hopkins) Rollins]

Arabis pycnocarpa M. Hopkins var. pycnocarpa, Creamflower Rockcress, S1/G5T5 (Boone 820502 TAWES) [Arabis hirsuta (L.) Scop. var. pycnocarpa (M. Hopkins) Rollins]

Armoracia rusticana G. Gaertn., B. Mey. \& Scerb., Horseradish, † (Smith 130 US)

[Armoracia lapathifolia Gilb.]

- Aurinia saxatilis (L.) Desv., Basket-of-gold, † Waif Reed (1964) [Alyssum saxatile L.] 
Barbarea verna (Mill.) Asch., Early Yellow Rocket, † (Longbottom 8504 DOV)

[Campe verna (Mill.) A. Heller]

Barbarea vulgaris W. T. Aiton, Yellow Rocket, † (Longbottom 5695 DOV)

[Campe barbarea (L.) W. Wight ex Piper; C. stricta (Andrz.) W. Wight ex Piper]

Berteroa incana (L.) DC., Hoary False Alyssum, † (Longbottom et al. $4049 \mathrm{DOV})$

Boechera burkii (Porter) Windham \& Al-Shehbaz, Burk's Smooth Rockcress (Reveal et al. 5379 MARY) [Arabis laevigata (Muhl. ex Willd.) Poir. var. burkii Porter; Borodinia burkii (Porter) P. J. Alexander \& Windham]

Boechera canadensis (L.) Al-Shehbaz, Sicklepod (Ebert \& Holt 145EH DOV)

[Arabis canadensis L.; Borodinia canadensis (L.) P. J. Alexander \& Windham]

Boechera dentata (Raf.) Al-Shehbaz \& Zarucchi, Short's Rockcress, S3/G5 (Terrell 4091 US)

[Arabis perstellata E. L. Braun var. shortii Fernald; A. shortii (Fernald) Gleason; Boechera shortii (Fernald) Al-Shehbaz; Borodinia dentata (Raf.) P. J. Alexander \& Windham;]

Boechera laevigata (Muhl. ex Willd.) Al-Shehbaz, Smooth Rockcress (McAvoy 2111 DOV)

[Arabis laevigata (Muhl. ex Willd.) Poir.; Borodinia laevigata (Muhl. ex Willd.) P. J. Alexander \& Windham]

Boechera missouriensis (Greene) Al-Shehbaz, Missouri Rockcress, S1/G5 (Baltars 2092 US)

[Arabis missouriensis Greene; Borodinia missouriensis (Greene) P. J. Alexander \& Windham]

Brassica juncea (L.) Coss., Chinese Mustard, † (Longbottom 9174 DOV)

[Brassica japonica Thunb.]

Brassica napus L., Rapeseed, † (Bowman 372 MARY)

Brassica nigra (L.) W. D. J. Koch, Black Mustard, † (Shetler \& Orli 2004.10 US)

Brassica oleracea L., Wild Cabbage, † (McAtee 2746 US)

Brassica rapa L., Bird's Rape, † (Longbottom 7102 DOV) [Brassica campestris L.]

Cakile edentula (Bigelow) Hook., American Searocket (Longbottom 3339 US)

Cakile maritima Scop., European Searocket, † (Baltars 2199 US) [Cakile cakile (L.) H. Karst.]

Calepina irregularis Thell., White Ball-mustard, † (Longbottom $10895 \mathrm{MO})$

Camelina microcarpa Andrz. ex DC., Little-seed False Flax, † (Leonard 2694 US)

[Camelina sativa ssp. microcarpa (Andrz. ex DC.) Em. Schmid]
Camelina sativa (L.) Crantz, Large-seed False Flax, $\uparrow$ (Rynet s.n. MARY)

Capsella bursa-pastoris (L.) Medik., Common Shepherd's Purse, $\dagger($ Knapp 2669 DOV)

[Bursa bursa-pastoris (L.) Britton]

Cardamine angustata O. E. Schulz, Slender Toothwort (McAvoy 5730 DOV)

[Dentaria heterophylla Nutt.]

Cardamine bulbosa (Schreb.) Britton, Sterns, \& Poggenb., Bulbous Bittercress (Knapp 2762 DOV)

[Cardamine rhomboidea DC.]

Cardamine concatenata (Michx.) Sw., Cutleaf Toothwort (Tatnall 4794 DOV)

[Dentaria laciniata Muhl.]

Cardamine diphylla (Michx.) Alph. Wood, Twoleaf Toothwort (Longbottom 1582 DOV)

Cardamine douglassii Britton, Purple Cress, S3/G5 (Smith 4973 US)

- Cardamine flexuosa With., Woodland Bittercress-see Excluded Flora section.

Cardamine hirsuta L., Hairy Bittercress, † (Longbottom 10834 DOV)

Cardamine impatiens L., Narrowleaf Bittercress, ‡(Knapp 30610 \& Harrison TAWES)

Cardamine longii Fernald, Long's Bittercress, S2/G3? (McAvoy $4090 \mathrm{DOV})$

Cardamine occulta Hornem., Hidden Bittercress, † (Cusick $37550 \mathrm{CM}$ )

Cardamine parviflora L. var. arenicola (Britton) O. E. Schulz, Small-flowered Bittercress (Knapp 506 DOV) [Cardamine arenicola Britton]

Cardamine pensylvanica Muhl. ex Willd., Pennsylvania Bittercress (McAvoy 3478 DOV)

- Cardamine pratensis L. var. palustris Wimm. \& Grab., Cuckoo Flower-see Excluded Flora section.

Cardamine rotundifolia Michx., American Bittercress, S3/G4 (Baltars 2015 US)

Coincya monensis (L.) Greuter \& Burdet, Star-mustard, † (Baltars 2809 US)

Conringia orientalis (L.) Dumort., Hare's-ear Mustard, † (Otis s.n. DOV)

- Descurainia pinnata (Walter) Britton ssp. pinnata, Pinnate Tansy-mustard, † Waif McAtee (1930)

[Sophia millefolia Rydb. ex Britton; S. pinnata (Walter) Howell]

- Descurainia sophia (L.) Webb ex Prantl, Herb Sophia, † Waif (Plitt 264 US)

- Diplotaxis siifolia Kuntz, Cone-hair Wallrocket, † Waif Reed (1964) 
Diplotaxis tenuifolia (L.) DC., Slim-leaf Wallrocket, † (Tatnall $1161 \mathrm{DOV})$

Draba ramosissima Desv., Branched Whitlow-grass (Longbottom et al. $4102 \mathrm{DOV})$

Draba verna L., Spring Draba, † (Longbottom 10873 DOV)

[Erophila verna (L.) Besser]

Eruca vesicaria (L.) Cav., Rocket-salad, † (Shoemaker s.n. US)

[Eruca sativa (L.) Mill.]

Erucastrum gallicum (Willd.) O. E. Schulz, Common Dogmustard, † (Baltars 3131 US)

[Brassica erucastrum L.]

- Erysimum asperum (Nutt.) DC., Western Wallflower-see Excluded Flora section.

[Cheirina aspera (Nutt.) Britton]

Erysimum capitatum (Douglas ex Hook.) Greene, Sand Dune Wallflower, †? (Reveal 7495 MARY)

Erysimum cheiranthoides L., Wormseed Mustard, † (Wells et al. 4349 US)

[Cheirinia cheiranthoides (L.) Link]

- Erysimum inconspicuum (S. Watson) MacMil., Small-flowered Prairie Wallflower-see Excluded Flora section.

Erysimum repandum L., Spreading Wallflower, † (Baltars 4046 US)

Hesperis matronalis L., Dame's Rocket, † (Windler \& Lombardo 3063 DOV)

Lepidium campestre (L.) R. Br., Field Peppergrass, † (Knapp 15-03 DOV)

Lepidium densiflorum Schrad. var. densiflorum, Dense-flowered Peppergrass, † (Longbottom 9191 DOV)

- Lepidium didymum L., Wartcress, † Waif Reed (1964)

[Carara didyma (L.) Britton; Coronopus didymus (L.) Sm.]

Lepidium draba L., Heart-pod Hoarycress, † (Longbottom 8718 DOV)

[Cardaria draba (L.) Desv.]

- Lepidium graminifolium L., Grassleaf Peppergrass, † Waif Reed (1964)

- Lepidium hyssopifolium Desv., Hyssopleaf Pepperwort, † Waif Reed (1964)

Lepidium oblongum Small, Veiny Pepperweed, † (Longbottom 12518 MARY)

- Lepidium perfoliatum L., Clasping Peppergrass, † Waif Reed (1964)

Lepidium ruderale L., Narrowleaf Peppergrass, † (Solomon 437 MARY)

Lepidium virginicum L., Poor-man's Peppergrass (Lombardo 3542 DOV)

Lobularia maritima (L.) Desv., Sweet Alyssum, † (Norton s.n. US) [Koniga maritima (L.) R. Br.]
Lunaria annua L., Annual Honesty, † (Knapp 16-03 DOV)

Lunaria rediviva L., Perennial Honesty, $†$ (Klein 143 MARY)

Microthlaspi perfoliatum (L.) F. K. Mey., Perfoliate Pennycress, † (Longbottom 18892 MARY)

[Thlaspi perfoliatum L.]

Nasturtium officinale R. Br., Watercress, $†$ (Longbottom et al. 7329 DOV)

[Rorippa nasturtium Beck; $R$. nasturtium-aquaticum (L.) Hayek; Sisymbrium nasturtium-aquaticum L.]

Neobeckia aquatica (Eaton) Greene, Lakecress, S1/G4? (Ward s.n. US)

[Armoracia aquatica (Eaton) Wiegand; A. lacustris (A. Gray) Al-Shehbaz \& V. M. Bates]

Planodes virginicum (L.) Greene, Virginia Rockcress (Vasey s.n. US)

[Arabis virginica (L.) Poir.; Cardamine virginica L.; Sibara virginica (L.) Rollins]

Raphanus raphanistrum L., Wild Radish, † (Baltars 2637 US)

Raphanus sativus L., Garden Radish, † (Redmond 537 DOV)

Rorippa palustris (L.) Besser, Bog Yellowcress (Longbottom 8837 DOV)

[Radicula palustris (L.) Moench; Rorippa islandica (Oeder) Borbás var. fernaldiana Butters \& Abbe; R. islandica var. hispida (Desv.) Butters \& Abbe; R. palustris var. hispida (Desv.) Rydb.]

Rorippa sessiliflora (Nutt.) Hitchc., Stalkless Yellowcress (Hitchcock s.n. US)

[Radicula sessiliflora (Nutt.) Greene]

- Rorippa sinuata (Nutt.) Hitchc., Spreading Yellowcress, † Waif Reed (1964)

Rorippa sylvestris (L.) Besser, Creeping Yellowcress, † (Lea 3314 DOV)

[Radicula sylvestris (L.) Druce]

- Sinapis alba L., White Mustard, † Waif Reed (1964) [Brassica alba (L.) Rabenh.; B. hirta Moench]

Sinapis arvensis L., Corn Mustard, † (Turck s.n. DOV) [Brassica kaber (DC.) L. C. Wheeler]

Sisymbrium altissimum L., Tumbleweed Mustard, † (Hotchkiss 5849 US)

[Norta altissima (L.) Britton]

- Sisymbrium loeselii L., Small Tumbleweed Mustard, † Waif (Norton s.n. MARY)

Sisymbrium officinale (L.) Scop., Hedge-mustard, † (Tidestrom 11807 DOV)

[Erysimum officinale L.] 
Teesdalia nudicaulis (L.) Aiton, Common Shepherd's-cress, † (Blake 11654 US)

Thlaspi alliaceum L., Roadside Pennycress, † (Davis 7265 \& Davis BALT)

Thlaspi arvense L., Field Pennycress, † (Leonard 19850 US)

Turritis glabra L., Tower Mustard (McDowell s.n. NY) [Arabis glabra (L.) Bernh.]

\section{BROMELIACEAE}

(Pineapple Family)

Tillandsia usneoides (L.) L., Spanish Moss, SX/G5 (H.S. [Sloane] 100:9 BM)-Reported by Brown et al. (1987) and known only from two collections made during the 1700 s. [Dendropogon usneoides (L.) Raf.]

\section{BUXACEAE}

(Boxwood Family)

- Pachysandra procumbens Michx., Allegheny Spurge-see Excluded Flora section.

Pachysandra terminalis Siebold \& Zucc., Asiatic Pachysandra, $\dagger$ (Grand 89-00453 DOV)

\section{CABOMBACEAE}

(Watershield Family)

Brasenia schreberi J. F. Gmel., Watershield (Tatnall 4985 DOV) [Brasenia purpurea (Michx.) Caspary]

Cabomba caroliniana A. Gray, Carolina Fanwort, $\ddagger$ (Steury 030629.1 US)

\section{CACTACEAE}

(Cactus Family)

Opuntia cespitosa Raf., Prickly-pear (Reed 121328 MO)

Opuntia bumifusa Raf. var. humifusa, Eastern Prickly-pear (Tatnall 3006 DOV)

Opuntia mesacantha Raf. ssp. mesacantha, Common Pricklypear (Reed $112805 \mathrm{MO})$

[Opuntia compressa J. F. Macbr.]

\section{CALYCANTHACEAE (Strawberry-shrub Family)}

Calycanthus floridus L., Sweetshrub, † (Oristian 6 MARY) [Butneria fertilis (Walter) Kearney; Calycanthus fertilis Walter; C. floridus var. glaucus (Willd.) Torr. \& A. Gray; C. floridus var. laevigatus (Willd.) Torr. \& A. Gray; C. nanus Loisel.]

\section{CAMPANULACEAE (Bellflower Family)}

Campanula americana L., Tall Bellflower (Randall Jr. 6 US) [Campanulastrum americanum (L.) Small]

- Campanula divaricata Michx., Appalachian Bellflower, - SH/G4-see Excluded Flora section.

Campanula rapunculoides L., Creeping Bellflower, † (Flemming 1187 US)
Campanula rotundifolia L., American Harebell, S2/G5 (Reed ¿o Shetler 88124 US)

- Jasione montana L., Sheep's-bit-see Excluded Flora section.

Lobelia canbyi A. Gray, Canby’s Lobelia, S2/G4 (Hirst 829 DOV)

Lobelia cardinalis L., Cardinal-flower (Tatnall 3482 DOV)

- Lobelia dortmanna L., Water Lobelia-see Excluded Flora section.

Lobelia elongata Small, Elongated Lobelia, S3/G4G5 (Hirst 831 DOV)

- Lobelia glandulosa Walters, Glade Lobelia-see Excluded Flora section.

Lobelia inflata L., Indian-tobacco (O’Neil s.n. DOV)

Lobelia nuttallii Roem. \& Schult., Nuttall's Lobelia (Knapp 908 DOV)

Lobelia puberula Michx., Downy Lobelia (Naczi 8863 DOV)

Lobelia siphilitica L., Great Blue Lobelia (Longbottom 10200 DOV)

Lobelia spicata Lam., Pale-spike Lobelia (Shriver s.n. MARY) [Lobelia bracteata Small; L. leptostachys A. DC; L. spicata var. campanulata McVaugh; L. spicata var. leptostachys (A. DC.) Mack. \& Bush; L. spicata var. scaposa McVaugh]

Palustricodon aparinoides (Pursh) Morin, Marsh Bellflower (Tatnall 1612 DOV)

[Campanula aparinoides Pursh]

Triodanis perfoliata (L.) Nieuwl., Claspingleaf Venus' Lookingglass (Redmond 137 DOV)

[Legousia perfoliata (L.) Britton; Specularia perfoliata (L.) DC.]

CANNABACEAE

(Hemp Family)

Cannabis sativa L., Marijuana, $†$ (Blake s.n. US)

- Celtis laevigata Willd., Sugarberry-see Excluded Flora section.

[Celtis mississippiensis Bosc]

Celtis occidentalis L., Common Hackberry (Kiltz 24 US) [Celtis occidentalis var. pumila (Pursh) A. Gray; C. pumila Pursh]

Celtis tenuifolia Nutt., Dwarf Hackberry (Hitchcock 12782 US) [Celtis georgiana Small; C. occidentalis var. georgiana (Small) H. E. Ahles; C. smallii Beadle]

Humulus japonicus Siebold \& Zucc., Japanese Hop, ‡ (Longbottom 6681 DOV)

Humulus lupuloides (E. Small) Tembrock, American Hop (Morong s.n. NY)

Humulus lupulus L., Common Hop, † (Topping 1414 US) 


\section{CAPRIFOLIACEAE}

(Honeysuckle Family)

Diervilla lonicera Mill., Northern Bush Honeysuckle (Frye ๘ Knapp 5535 TAWES)

[Diervilla diervilla (L.) MacMill.]

Dipsacus fullonum L., Fuller's Teasel, † (Topping s.n. US)

[Dipsacus sylvestris Huds.]

Dipsacus laciniatus L., Cutleaf Teasel, † (Steury 030824.1 US)

Dipsacus sativus (L.) Honckeny, Indian Teasel, † (Cusick 38000 CM)

- Knautia arvensis (L.) J. M. Coult., Blue-button-see Excluded Flora section.

- Linnaea borealis L., Twinflower-see Excluded Flora section.

Lonicera canadensis Bartram ex Marshall, American Fly Honeysuckle, S1/G5 (Thompson Photo Voucher TAWES)

Lonicera dioica L., Mountain Honeysuckle (Frye \& Frye 5914 TAWES)

[Lonicera dioica var. glaucescens (Rydb.) Butters]

Lonicera fragrantissima Lindl. \& Paxton, Sweet-breath-ofspring $\ddagger$ (Hill 9835 MARY)

[Xylosteon fragrantissimum (Lindl. \& Paxton) Small]

Lonicera japonica Thunb., Japanese Honeysuckle, $\ddagger$ (Longbottom $8929 \mathrm{DOV})$

Lonicera maackii (Rupr.) Herder, Amur Honeysuckle, ‡ (Frye \& Baranski 4047 TAWES)

Lonicera morrowii A. Gray, Morrow’s Honeysuckle, $\ddagger$ (Bennett $20 \mathrm{DOV})$

Lonicera sempervirens L. var. sempervirens, Trumpet Honeysuckle (Frye 5531 TAWES)

[Phenianthus sempervirens (L.) Raf.]

Lonicera standishii Jacques, Standish’s Honeysuckle, $\ddagger$ (Fosberg \& Rainey 55578 US)

Lonicera tatarica L., Tatarian Honeysuckle, $\ddagger$ (Penson s.n. MARY)

Lonicera xylosteum L., Dwarf Honeysuckle, †? (Mallnoff s.n. MARY)

Symphoricarpos albus (L.) S. F. Blake var. albus, Common Snowberry, S1/G5T5 (Frye \& Thompson 1071 TAWES)

Symphoricarpos albus (L.) S. F. Blake var. laevigatus (Fernald) S. F. Blake, Common Snowberry, † (Earley s.n. Frostburg State University Herbarium)

Symphoricarpos occidentalis Hook., Northern Snowberry, $\dagger$ [s. coll., s.n. MARY)

Symphoricarpos orbiculatus Moench, Coralberry, † (Tidestrom 12203 US)

[Symphoricarpos symphoricarpos (L.) MacMill.]

Triosteum angustifolium L., Yellowleaf Tinker's-weed, S1/G5 (Terrell 4161 US)
Triosteum aurantiacum E. P. Bicknell, Coffee Tinker's-weed (Van Eseltine 83 US)

Triosteum perfoliatum L., Perfoliate Tinker's-weed (Ward s.n. US)

- Valeriana officinalis L., Common Valerian-see Excluded Flora section.

Valeriana pauciflora Michx., Valerian, S1/G4 (Tatnall 4515 DOV)

- Valerianella chenopodiifolia DC., Goosefoot Cornsalad, - S1/G4-see Excluded Flora section.

Valerianella locusta (L.) Lat., European Cornsalad, † (Coville s.n. US)

[Valerianella olitoria Pollich]

Valerianella radiata (L.) Dufr., Beaked Cornsalad (Baltars 591 US)

[Valerianella woodsiana (Torr. \& A. Gray) Walpers]

Valerianella umbilicata (Sull.) Alph. Wood, Navel-shaped Cornsalad, SH/G5 (Baltars 592 US)

[Valerianella intermedia Dyal; V. patellaria Alph. Wood]

CARYOPHYLLACEAE

(Pink Family)

Agrostemma githago L., Common Corncockle, $\uparrow$ (Jensen s.n. US)

Arenaria leptoclados (Rchb.) Guss., Slender Sandwort (Norton s.n. MARY)

Arenaria serpyllifolia L., Thymeleaf Sandwort, † (Frye \& Baranski 5395 TAWES)

Cerastium arvense L. ssp. arvense, Mouse-ear Chickweed, † (Walker 516 MARY)

Cerastium arvense L. ssp. strictum (L.) Ugborogho, Mouse-ear Chickweed (Tatnall 5213 DOV)

Cerastium brachypetalum Pers., Gray Chickweed, † (Longbottom $8582 \mathrm{DOV})$

Cerastium fontanum Baumg. ssp. vulgare (Hartm.) Greuter \& Burdet, Common Mouse-ear

Chickweed, † (Fosberg 18160 US) [Cerastium vulgatum L.]

Cerastium glomeratum Thuill., Sticky Mouse-ear Chickweed, † (Otis s.n. DOV)

[Cerastium viscosum L.]

Cerastium nutans Raf. var. nutans, Nodding Chickweed (Ebert \& Holt $312 \mathrm{PH})$ [Cerastium longepedunculatum Muhl. ex Britton]

Cerastium pumilum M. A. Curtis, European Chickweed, † (Jansen s.n. US)

Cerastium semidecandrum L., Five-stamen Mouse-ear Chickweed, † (Hill 13792 MARY)

Cerastium velutinum Raf. var. velutinum, Field Chickweed (Frye 5905 TAWES) 
Cerastium velutinum Raf. var. villosissimum (Pennell) J. K. Morton, Octoraro Creek Chickweed, S1/G5T1 (Long \& Bartram 1259 US)

[Cerastium arvense L. var. villosum Hollick \& Britton]

- Corrigiola litoralis L., European Corrigiola-see Excluded Flora section.

Dianthus armeria L., Deptford Pink, † (Longbottom 6206 DOV) Dianthus barbatus L., Sweet William, † (Leonard 19880 US)

- Dianthus deltoides L., Maiden Pink-see Excluded Flora section.

Dianthus plumarius L., Garden Pink, † (Baltars 512 US)

Gypsophila paniculata L., Tall Baby's-breath, † (Longbottom et al. 3319 MARY)

- Gypsophila pilosa Huds., Turkish Baby's-breath, † Waif Reed (1964)

[Saponaria porrigens L.]

- Herniaria glabra L., Smooth Burstwort, † Waif Reed (1964)

Herniaria hirsuta L. var. cinerea (DC.) Loret \& Barrandon, Gray Burstwort, † (Hill \& Kratz 14380 Assateague Island National Seashore Herbarium)

[Herniaria cinerea DC.; H. incana Lam.]

Holosteum umbellatum L., Jagged Chickweed, † (Longbottom 8455 DOV)

Honckenya peploides (L.) Ehrh. ssp. robusta (Fernald) Hultén, Eastern Seabeach Sandwort, S2/G5T4 (Lea 2271 TAWES)

Minuartia caroliniana (Walter) Mattf., Pine Barren Sandwort, - S1/G5 (Harrison 000620.1 TAWES)

[Arenaria caroliniana Walter; Sabulina caroliniana (Walter) Small]

Minuartia glabra (Michx.) Mattf., Appalachian Sandwort, - S1/G4 (Wiegand Photo Voucher MPA) [Mononeuria glabra (Michx.) Dillenb. \& Kadereit]

Minuartia michauxii (Fenzl) Farw. var. michauxii, Michaux's Stitchwort, S2/G5T4 (Frye \& Harrison s.n. TAWES) [Arenaria michauxii Hook. f.; A. stricta Michx.; Sabulina stricta (Michx.) Small]

Moebringia lateriflora (L.) Fenzl, Grove Sandwort, S1/G5 (Frye 5513 TAWES)

Moenchia erecta (L.) G. Gaertn., B. Mey. \& Scherb. ssp. erecta, Upright Chickweed, † (Frye \& Berdine 1087 TAWES)

Myosoton aquaticum (L.) Moench, Giant Chickweed, $\uparrow$ (Baltars 340 US)

[Stellaria aquatica (L.) Scop.]

- Paronychia argyrocoma (Michx.) Nutt., Silverling-see Excluded Flora section.
Paronychia canadensis (L.) Wood, Forked Nailwort (Knapp 2799 DOV)

[Anychia canadensis (L.) Britton, Sterns, \& Poggenb.; A. dichotoma Michx.; Paronychia dichotoma (Michx.) A. Nelson]

Paronychia fastigiata (Raf.) Fernald, Cluster-stemmed Nailwort (Fosberg 18461 US)

[Anychia polygonoides Raf.; Paronychia fastigiata var. nuttallii (Small) Fernald; P. fastigiata var. paleacea Fernald]

Paronychia montana Pax \& K. Hoffm., Mountain Nailwort (Frye GRSFBD 671 TAWES)

[Paronychia fastigiata (Raf.) Fernald var. pumila (Alph. Wood) Fernald]

Paronychia virginica Spreng. var. virginica, Yellow Nailwort, S1/G4T1Q (Frye \& Knapp 5543 TAWES)

Petrorhagia prolifera (L.) P. W. Ball \& Heywood, Childing Pink, † (Steury 020713.6 US)

[Dianthus prolifer L.; Tunica prolifera (L.) Scop.]

Sagina apetala Ard., Small-flowered Pearlwort, † (Blake 11658 NA)

Sagina decumbens (Elliott) Torr. \& A. Gray ssp. decumbens, Small-flowered Pearlwort (Tatnall 1773 DOV)

[Sagina decumbens var. smithii (A. Gray) S. Watson]

Sagina procumbens L., Procumbent Pearlwort, † (Arsène 223 US)

Saponaria officinalis L., Bouncing Bet, † (Longbottom 9727 DOV)

[Lychnis saponaria Jess.]

Scleranthus annuus L., Annual Knawel, † (Knapp 2656 DOV)

Silene antirrbina L., Sleepy Catchfly, † (McAvoy 6746 DOV)

Silene armeria L., Thrift Catchfly, † (Hambleton s.n. US)

Silene caroliniana Walter ssp. pensylvanica (Michx.) R. T. Clausen, Carolina Pink (Knapp 1171 DOV)

- Silene chalcedonica E. H. L. Krause, Burning Love-see Excluded Flora section.

- Silene conica L., Striate Catchfly-see Excluded Flora section.

- Silene conoidea L., Conoid Catchfly-see Excluded Flora section.

Silene coronaria (L.) Clairv., Rose Campion, † (Longbottom et al. 7413 DOV)

[Lychnis coronaria (L.) Desr.]

- Silene csereii Baumg., Balkan Catchfly, † Waif Reed (1964)

Silene dichotoma Ehrh., Forked Catchfly, † (Benton s.n. US)

Silene dioica (L.) Clairv., Red Catchfly, † (Baltars 696 US) [Lychnis dioica L.]

- Silene drummondii Hook., Drummond's Campion-see Excluded Flora section.

[Lychnis drummondii (Hook.) S. Watson] 
Silene flos-cuculi (L.) Greuter \& Burdet, Ragged Robin, † (Knapp 5404 MARY)

[Lychnis flos-cuculi L.]

Silene latifolia Poir., Bladder Campion, † (Longbottom 6499 DOV)

[Lychnis alba Mill.; Silene alba (Mill.) Krause; S. latifolia ssp. alba (Mill.) Greuter \& Burdet]

Silene nivea (Nutt.) Muhl. ex Ott, Snowy Campion, S1/G4? (Baltars 616 US)

Silene noctiflora L., Night-flowering Catchfly, † (Standley 11726 US)

Silene stellata (L.) W. T. Aiton, Starry Catchfly (McAvoy 6280 DOV)

- Silene virginica L., Fire Pink-see Excluded Flora section.

Silene vulgaris (Moench) Garcke, Maiden's-tears, † (Deignan 357 US)

Spergula arvensis L., Corn Spurrey, † (Smith 476 DOV)

Spergula morisonii Boreau, Morison's Spurrey, † (Steury 020420.1 US)

Spergularia marina (L.) Griseb., Saltmarsh Sandspurry (House 1453 US)

[Spergularia salina J. Presl \& C. Presl; Tissa marina (L.) Britton]

Spergularia media (L.) Griseb., Greater Seaspurry (Cusick $38003 \mathrm{CM})$

Spergularia rubra (L.) J. Presl \& C. Presl, Purple Chickweed, $\dagger$ (Phillips s.n. DOV)

[Tissa rubra (L.) Britton]

Stellaria alsine Grimm, Trailing Chickweed, S1/G4G5 (Steury 040501.2 US)

- Stellaria borealis Bigelow var. borealis, Boreal Chickweedsee Excluded Flora section.

Stellaria graminea L., Little Chickweed, † (Frye 3088 TAWES)

Stellaria longifolia Muhl. ex Willd., Longleaf Chickweed (Longbottom $9310 \mathrm{DOV}$ )

Stellaria longipes Goldie ssp. longipes, Longstalk Chickweed, †? (Baltars 715 US)

Stellaria media (L.) Vill., Medium Chickweed, † (Longbottom $10862 \mathrm{DOV})$

[Alsine media L.]

- Stellaria neglecta (Lej.) Weihe, Common Chickweed-see Excluded Flora section.

Stellaria pubera Michx., Giant Chickweed (Knapp 513 DOV) [Alsine pubera (Michx.) Britton]

- Vaccaria hispanica (Mill.) Rauschert, Cowcockle, † Waif Reed (1964)

[Saponaria vaccaria L.; Vaccaria vaccaria (L.) Britton]

CELASTRACEAE

(Bittersweet Family)

Celastrus orbiculatus Thunb., Oriental Bittersweet, $\ddagger$ (Longbottom 10764 DOV)
Celastrus scandens L., Climbing Bittersweet (Tatnall 4961 DOV)

Euonymus alatus (Thunb.) Siebold, Burning Bush, $\ddagger$ (Wells \& Shetler s.n. US)

Euonymus americanus L., Bursting-heart (Tatnall 2949 DOV)

Euonymus atropurpurea Jacq., Wahoo (Otis s.n. DOV)

Euonymus europaeus L., European Spindletree, † (Reveal 8147 MARY)

Euonymus fortunei (Turcz.) Hand.-Mazz. var. radicans (Siebold ex Miq.) Rehder, Winter Creeper, $\ddagger$ (Longbottom 471 DOV) [Euonymus radicans Siebold ex Miq.]

Parnassia asarifolia Vent., Kidneyleaf Grass-of-Parnassus, - S1/G4 (Stango Photo Voucher TAWES)

Paxistima canbyi A. Gray, Canby’s Mountain-lover, S1/G2? (Boone s.n. TAWES)

[Pachistima canbyi A. Gray]

\section{CERATOPHYLLACEAE}

(Hornwort Family)

Ceratophyllum demersum L., Common Hornwort (McAvoy $5920 \mathrm{DOV})$

Ceratophyllum echinatum A. Gray, Prickly Hornwort, S2?/G4 (Steury 980515.7 US)

CISTACEAE

(Rockrose Family)

Crocanthemum bicknellii (Fernald) Barnhart, Plains Frostweed, - S1/G5 (Holt \& Ebert s.n. TAWES)

[Helianthemum bicknellii Fernald]

Crocanthemum canadense (L.) Britton, Canada Frostweed (Frye 3098 TAWES)

[Helianthemum canadense (L.) Michx.]

Crocanthemum propinquum (E. P. Bicknell) E. P. Bicknell, Low Frostweed (Knapp 39-03 TAWES)

[Helianthemum propinquum E. P. Bicknell]

Hudsonia ericoides L., Golden Heather, S1/G4 (Sipple 2032 DOV)

Hudsonia tomentosa Nutt., Sand Heather (Knapp 53-02 TAWES)

Lechea maritima Legg. ex Britton var. virginica Hodgdon, Virginian Beach Pinweed, S3/G5T3Q (Hill 15741 US)

Lechea minor L., Thymeleaf Pinweed (Kearney, Jr. s.n. US)

Lechea mucronata Raf., Hairy Pinweed (Longbottom 20051 MARY)

[Lechea villosa Elliott]

Lechea pulchella Raf., Leggett's Pinweed (Uhler 37 US) [Lechea leggettii Britton \& Hollick; L. pulchella var. moniliformis (E. P. Bicknell) Mohlenbr.]

Lechea racemulosa Michx., Illinois Pinweed (Strong \& Simmons 3201 US)

Lechea tenuifolia Michx., Slender Pinweed, SH/G5 (Leonard \& Killip 751 US) 


\section{CLEOMACEAE}

(Cleome Family)

Cleome hassleriana Chodat, Spiderflower, † (Lea 2273 US) [Cleome houtteana Schltdl.; C. spinosa Jacq.; Neocleome spinosa (Jacq.) Small; Tarenaya hassleriana (Chodat) Iltis]

Cleome ornithopodioides L., Bird Spiderflower, † (Knapp 4151 $M A R Y)$

- Cleome viscosa L., Asian Spiderflower, † Waif Reed (1964) [Corynandra viscosa (L.) Cochrane \& Iltis]

Polanisia dodecandra (L.) DC. ssp. dodecandra, Common Clammyweed, S1/G5T5 (Shull 113 US)

[Polanisia trachysperma Torr. \& A. Gray]

CLETHRACEAE (White Alder/Pepperbush Family)

Clethra alnifolia L., Coastal Sweet Pepperbush (Simmons \& Strong 1430 US)

COLCHICACEAE

(Meadow Saffron Family)

- Colchicum autumnale L., Meadow Saffron-see Excluded Flora section.

Uvularia grandiflora Sm., Large-flowered Bellwort, S1/G5 (Reveal \& Brown 5150 MARY)

Uvularia perfoliata L., Perfoliate Bellwort (Chase 6877 US)

Uvularia puberula Michx., Carolina Bellwort (Cusick 22462 MARY)

[Uvularia pudica Fernald]

Uvularia sessilifolia L., Straw-lily (Hotchkiss 5089 US)

\section{COMMELINACEAE}

(Spiderwort Family)

Commelina caroliniana Walter, Indian Dayflower, † (Reed 41364 US)

Commelina communis L., Common Dayflower, $†$ (Fosberg 18496 US)

[Commelina communis var. ludens (Miq.) C. B. Clarke]

- Commelina diffusa Burm. f., Creeping Dayflower, † Waif Reed (1964)

[Commelina longicaulis Jacq.]

Commelina erecta L., Erect Dayflower, S3/G5 (Hitchcock 15748 US)

[Commelina angustifolia Michx.; C. crispa Wooton; C. erecta var. angustifolia (Michx.) Fernald]

Commelina virginica L., Virginia Dayflower (Steury 980807.8 US) [Commelina hirtella Vahl]

- Cuthbertia graminea Small, Grassleaf Roseling—see Excluded Flora section.

[Callisia graminea (Small) G. C. Tucker; Tradescantia rosea Vent. var. graminea (Small) E. S. Anderson \& Woodson]

- Cuthbertia rosea (Vent.) Small, Common Roseling-see Excluded Flora section.

[Callisia rosea (Vent.) D. R. Hunt; Tradescantia rosea Vent.]
Murdannia keisak (Hassk.) Hand.-Mazz., Marsh Dewflower, $\ddagger$ (Hill 15924 US)

[Aneilema keisak Hassk.]

Tradescantia obiensis Raf., Smooth Spiderwort, † (Samars 43 MARY)

[Tradescantia incarnata Small; T. obiensis var. foliosa (Small) MacRoberts; T. reflexa Raf.]

Tradescantia virginiana L., Virginia Spiderwort (Baltars 710 US) [Tradescantia brevicaulis Raf.]

CONVOLVULACEAE

(Morning-glory Family)

Calystegia pubescens Lindl., Japanese False Bindweed, $\dagger$ (Pollard s.n. US)

Calystegia sepium (L.) R. Br., Hedge False Bindweed, † (Longbottom 9962 DOV)

[Calystegia sepium ssp. americana (Sims) Brummitt; C. sepium ssp. angulata Brummitt; C. sepium ssp. appalachiana Brummitt; Convolvulus nashii House; C. sepium L.]

Calystegia silvatica (Kit) Griseb. ssp. fraterniflora (Mack. \& Bush) Brummitt, Short-stalk False Bindweed (Killip 31056 US)

[Convolvulus sepium L. var. fraterniflorus Mack. \& Bush]

Calystegia spithamaea (L.) R. Br. ssp. spithamaea, Low Bindweed, S2/G4G5T4T5 (Tepfer 1330 MARY)

Calystegia spithamaea (L.) R. Br. ssp. stans (Michx.) Brummitt, Shale Bindweed (Brown s.n. MARY)

[Calystegia spithamaea ssp. purshiana (Wherry) Brummitt; Convolvulus purshianus Wherry; C. spithamaeus L.]

Convolvulus arvensis L., Field Bindweed, $\ddagger$ (Standley 11798 US) [Convolvulus incanus auct. non Vahl; Strophocaulos arvensis (L.) Small]

- Convolvulus wallichianus Spreng., Wallich's Bindweed-see Excluded Flora section.

Cuscuta campestris Yunck., Five-angled Dodder (Leonard \& Leonard 15994 US)

[Cuscuta arvensis Beyr.; C. pentagona Engelm.]

-Cuscuta cephalanthi Engelm., Buttonbush Dodder-see Excluded Flora section.

Cuscuta compacta Juss. var. compacta, Sessile Dodder (Strong \& Simmons 3285 US)

Cuscuta coryli Engelm., Hazel Dodder, S1/G5 (Hall JH2014.09.28.08 MARY)

Cuscuta cuspidata Engelm., Cusp Dodder, † (Mocvananua s.n. DOV)

- Cuscuta epilinum Weihe, Flax Dodder-see Excluded Flora section.

- Cuscuta epithymum (L.) L., Clover Dodder-see Excluded Flora section. 
Cuscuta gronovii Willd. ex Roem. \& Schult., Gronovius Dodder (Baltars 3248 US)

[Cuscuta gronovii var. latiflora Engelm.]

Cuscuta indecora Choisy var. indecora, Bigseed Alfalfa Dodder, S2?/G5T2T4 (Baltars 4008 US)

[Cuscuta indecora var. neuropetala (Engelm.) Hitchc.]

Cuscuta polygonorum Engelm., Smartweed Dodder, S1/G5 (Shull s.n. US)

Cuscuta rostrata Shuttlew. ex Engelm. \& A. Gray, Beaked Dodder, S1/G4 (Feller s.n. TAWES)

- Cuscuta suaveolens Ser., Fringed Dodder, † Waif (Hillman s.n. NY)

- Dichondra carolinensis Michx., Carolina Pony's-foot-see Excluded Flora section.

[Dichondra evolvulacea (L. f.) Britton]

- Dichondra micrantha Urb., Asian Pony's-foot, † Waif Reed (1964)

[Dichondra repens J. R. Forst. \& G. Forst.]

- Evolvulus filipes Mart., Needle Dwarf Morning-glory, † Waif Reed (1964)

Ipomoea coccinea L., Red Morning-glory, †? (Steward s.n. US) [Quamoclit coccinea (L.) Moench]

Ipomoea hederacea Jacq., Ivyleaf Morning-glory, †? (Walker 3851 US)

[Ipomoea barbigera Sweet; I. hederacea var. integriuscula A. Gray; Pharbitis barbigera (Sweet) G. Don; P. hederacea (Jacq.) Choisy]

Ipomoea lacunosa L., Whitestar, † (Longbottom 11851 US)

- Ipomoea nil (L.) Roth, White-edge Morning-glory, † Waif Reed (1964)

Ipomoea pandurata (L.) G. Mey., Big-root Morning-glory (Hotchkiss 5551 US)

Ipomoea purpurea (L.) Roth, Common Morning-glory, $\ddagger$ (Walker 3852 US)

[Pharbitis purpurea (L.) Voigt]

- Ipomoea quamoclit L., Cypress-vine-see Excluded Flora section [Ipomoea vulgaris Choisy]

CORNACEAE

(Dogwood Family)

Cornus alternifolia L. f., Alternate-leaf Dogwood (Jones s.n. US) [Swida alternifolia (L. f.) Small]

Cornus amomum Mill., Silky Dogwood (Uhler s.n. US) [Swida amomum (Mill.) Small]

Cornus canadensis L., Dwarf Dogwood, S1/G5 (Boone s.n. TAWES)

[Chamaepericlymenum canadense (L.) Asch. \& Craebn.]

Cornus florida L., Flowering Dogwood (Smith 4975 US)

[Cynoxylon floridum (L.) Small]

Cornus obliqua Raf., Pale Dogwood (Thompson 545 MARY)

[Swida obliqua (Raf.) Moldenke]
Cornus racemosa Lam., Gray Dogwood (McAtee 1594 US) [Swida racemosa (Lam.) Moldenke]

Cornus rugosa Lam., Roundleaf Dogwood, S1/G5 (Norton s.n. MARY)

[Swida rugosa (Lam.) Moldenke]

Cornus stolonifera Michx., Red-osier Dogwood (Hill 10828 MARY)

[Cornus alba L.; C. sericea L.; Swida stolonifera (Michx.) Rydb.]

Cornus stricta Lam., Stiff Dogwood (Ebert \& Holt 144EH DOV)

[Cornus foemina Mill.; Swida stricta (Lam.) Small]

CRASSULACEAE

(Stonecrop Family)

Crassula aquatica (L.) Schönland, Water Pygmyweed, S1/G5 (Knapp et al. 4299 TAWES)

[Tillaea aquatica L.; Tillaeastrum aquaticum (L.) Britton]

Hylotelephium telephioides (Michx.) H. Ohba, Allegheny Stonecrop (Steele s.n. US)

[Anacampseros telephioides (Michx.) Haw.; Sedum telephioides Michx.]

Hylotelephium telephium (L.) H. Ohba, Garden Stonecrop, † (Hill 17449 MARY)

[Sedum purpureum (L.) Schult.; S. telephium L.]

- Sedum acre L., Gold Moss, † Waif (Norton s.n. MARY)

Sedum glaucophyllum R. T. Clausen, Cliff Stonecrop, S2/G4 (Knapp \& Thompson 3364 TAWES)

Sedum sarmentosum Bunge, Stringy Stonecrop, † (Steury 020517.1 US)

Sedum ternatum Michx., Woodland Stonecrop (Leonard 3108 US)

CUCURBITACEAE

(Cucumber Family)

Citrullus lanatus (Thunb.) Matsum. \& Nakai var. lanatus, Watermelon, † (Longbottom \& Van Velsir 10466 DOV) [Citrullus citrullus Farw.; Citrullus vulgaris Schrad.]

- Ctenolepis cerasiformis (Stocks) Naud., Ctenolepis, † Waif Reed (1964)

[Blastania fimbristipula Kotschy \& Peyr.]

Echinocystis lobata (Michx.) Torr. \& A. Gray, Wild Mock Cucumber (Hitchcock 15702 US)

Melothria pendula L., Creeping Cucumber (Longbottom 9910 DOV)

[Melothria microcarpa Shuttlew. ex Small; M. nashii Small; M. pendula var. aspera Cogn.]

Sicyos angulatus L., One-seeded Bur Cucumber (Taylor s.n. US) 


\section{CYPERACEAE}

(Sedge Family)

Bolboschoenus fluviatilis (Torr.) Soják, River Bulrush (Longbottom et al. 3080 US)

[Schoenoplectus fluviatilis (Torr.) M. T. Strong; Scirpus fluviatilis (Torr.) A. Gray]

- Bolboschoenus maritimus (L.) Palla ssp. paludosus (A. Nelson) T. Koyama, Saltmarsh Bulrush-see Excluded Flora section. [Scirpus paludosus A. Nelson]

Bolboschoenus novae-angliae (Britton) S. G. Sm., New England Bulrush, S2/G5T5 (Baltars 3587 MARY)

[Schoenoplectus novae-angliae (Britton) M. T. Strong; Scirpus novae-angliae Britton; S. cylindricus (Torr.) Britton]

Bolboschoenus robustus (Pursh) Soják, Sturdy Bulrush (Knapp 2191 DOV)

[Schoenoplectus robustus (Pursh) M. T. Strong; Scirpus robustus Pursh]

Bulbostylis capillaris (L.) Kunth ex C. B. Clarke, Densetuft Hairsedge (Hotchkiss 5609 US)

[Bulbostylis capillaris var. isopoda Fernald; Stenophyllus capillaris (L.) Britton]

- Bulbostylis hispidula (Vahl) R. W. Haines, Stiffhair Bulrush, † Waif Reed (1964) as B. hirta Thunb.

Carex abscondita Mack., Thicket Sedge (Naczi 8868 DOV)

Carex acutiformis Ehrh., Lesser Pond Sedge, † (Frye \& Longbottom 5579 TAWES)

Carex aestivalis M. A. Curtis ex A. Gray, Summer Sedge, S1/G4 (Boone 840808 TAWES)

Carex aggregata Mack., Glomerate Sedge (Naczi 8052 DOV)

Carex alata Torr., Broadwing Sedge (Lea 1679 TAWES)

Carex albicans Willd. ex Spreng. var. albicans, Whitetinge Sedge (Knapp 2376 DOV)

[Carex artitecta Mack.]

Carex albicans Willd. ex Spreng. var. emmonsii (Dewey ex Torr.) Rettig, Emmons' Sedge (Knapp 2381 DOV)

[Carex emmonsii Dewey ex Torr.)

Carex albolutescens Schwein., Greenwhite Sedge (Naczi 9294 DOV)

Carex albursina E. Sheld., White Bear Sedge, S3/G5 (Wixted \& Raymond s.n. TAWES)

Carex amphibola Steud., Eastern Narrowleaf Sedge (Naczi 8043 $\mathrm{NY}$ )

Carex annectens E. P. Bicknell, Yellowfruit Sedge (Naczi 9293 DOV)

[Carex annectens var. xanthocarpa (Kük.) Wiegand]

Carex appalachica J. M. Webber \& P. W. Ball, Appalachian Sedge, S1?/G4 (Thompson 830728 TAWES)

- Carex aquatilis Wahlenb., Water Sedge-see Excluded Flora section.

Carex arctata Boott., Drooping Woodland Sedge (Lea 2681 TAWES)

Carex arenaria L., Sand Sedge, † (Plitt 402 US)
Carex argyrantha Tuck. ex Dewey, Hay Sedge, S3/G5 (Frye 5291 TAWES)

Carex atlantica L. H. Bailey ssp. atlantica, Prickly Bog Sedge (Otis s.n. DOV)

[Carex incomperta E. P. Bicknell]

Carex atlantica L. H. Bailey spp. capillacea (L. H. Bailey) Reznicek, Howe's Sedge (McAvoy 2450 DOV) [Carex howei Mack.]

Carex austrina (Small) Mack., Southern Sedge, † (McAvoy 3591 DOV)

Carex baileyi Britton, Bailey's Sedge (Lea 2745 DOV)

Carex barrattii Schwein. \& Torr., Barratt's Sedge, S3/G4 (Naczi 9273 DOV)

Carex blanda Dewey, Eastern Woodland Sedge (Naczi 8048 DOV)

Carex brevior (Dewey) Mack., Shortbeak Sedge, †? (Steury 020524.1 US)

Carex bromoides Schkuhr ex Willd. ssp. bromoides, Brome-like Sedge (Naczi 8064 NY)

Carex brunnescens (Person) Poir, Brownish Sedge, S3/G5 (Longbottom et al. 7394 DOV)

Carex bullata Schkuhr ex Willd., Button Sedge, S3/G5 (Naczi 8947 DOV)

Carex bushii Mack., Bush's Sedge (Naczi 10350 DOV)

Carex buxbaumii Wahlenb., Buxbaum's Sedge, S2/G5 (Knapp 1405 DOV)

Carex canescens L. var. canescens, Silvery Sedge (Steury 040501.3 US)

Carex canescens L. var. disjuncta Fernald, Remote Silvery Sedge (McAvoy 4781 DOV)

Carex careyana Torr., Carey's Sedge, S1/G4G5 (Frye \& Knapp 5533 TAWES)

Carex caroliniana Schwein., Carolina Sedge (Longbottom 7508 DOV)

- Carex caryophyllea Latourr., Vernal Sedge-see Excluded Flora section.

- Carex cephaloidea (Dewey) Dewey, Thin-leaved Sedge-see Excluded Flora section.

Carex cephalophora Muhl. ex Willd., Oval-headed Sedge (McAvoy $4654 \mathrm{DOV})$

Carex collinsii Nutt., Collins' Sedge (Hirst \& Stasz s.n. DOV)

Carex communis L. H. Bailey var. communis, Fibrousroot Sedge (Naczi 11058 DOV)

Carex comosa Boott, Longhair Sedge (Knapp \& Maloof 60-01 DOV)

Carex complanata Torr. \& Hook., Hirsuit Sedge (Knapp 1312 DOV)

Carex conjuncta Boott, Soft Fox Sedge (Lea 3043 DOV)

Carex conoidea Willd., Openfield Sedge, S1/G5 (Naczi 10349 $\mathrm{NY}$ )

Carex corrugata Fernald, Prune-fruit Sedge (Lea 1177 DOV)

Carex crinita Lam. var. brevicrinis Fernald, Symmetrical Fringed Sedge (Lea 3005 DOV) 
Carex crinita Lam. var. crinita, Fringed Sedge (McAvoy 3960 DOV)

Carex cristatella Britton, Crested Sedge, S1?/G5 (Lea 1220 TAWES)

Carex davisii Schwein. \& Torr., Davis' Sedge, S1/G4 (Lea 1815 TAWES)

Carex debilis Michx. var. debilis, White-edge Sedge (Lea 2150 DOV)

[Carex allegheniensis Mack.; C. debilis var. intercursa Fernald; C. debilis var. prolixa L. H. Bailey; C. debilis var. pubera A. Gray]

Carex debilis Michx. var. rudgei L. H. Bailey, Flexuose Whitesedge (Longbottom 6066 DOV)

[Carex flexuosa Muhl. ex Willd.]

Carex decomposita Muhl., Cypress-knee Sedge, S1/G3 (Wright \& Fleming s.n. US)

Carex diandra Schrank, Lesser Tussock Sedge, S1/G5 (Frye o Lea s.n. DOV)

Carex digitalis Willd. var. digitalis, Slender Wood Sedge (Naczi $8063 \mathrm{NY}$ )

Carex digitalis Willd. var. macropoda Fernald, Long-spiked Slender Wood Sedge, S1?/G5TNR (McAvoy 6119 DOV)

Carex distans L., Distant Sedge † (Hotchkiss \& Ubler 7218 US)

Carex divisa Huds., Separated Sedge † (Hermann 10701 US)

- Carex divulsa Stokes, Grassland Sedge-see Excluded Flora section.

Carex eburnea Boott, Ivory Sedge, S1/G5 (Thompson s.n. TAWES)

Carex echinata Murray ssp. echinata, Star Sedge, S3/G5T5 (Frye \& Harrison s.n. DOV)

[Carex angustior Mack.; C. cephalantha (L. H. Bailey) E. P. Bicknell]

Carex emoryi Dewey, Emory's Sedge, S3/G5 (Lea 2448 DOV)

Carex extensa Goodenough, Longbract Sedge, † (Lea 857 Assateague Island National Seashore Herbarium)

Carex exilis Dewey, Meager Sedge, S1/G5 (Sipple 2127 DOV)

Carex festucacea Schkuhr, Festuca Sedge (Naczi 9292 DOV)

Carex fissa Mack. var. fissa, Hammock Sedge † (Longbottom 9355 DOV)

- Carex foenea Willd., Dryspike Sedge-see Excluded Flora section.

[Carex siccata Dewey]

Carex folliculata L., Northern Long Sedge (Knapp 2448 DOV)

Carex frankii Kunth, Frank's Sedge (Knapp 2189 DOV)

Carex fraseriana Ker Gawl., Fraser's Sedge, S1/G4 (Longbottom et al. 6000 DOV)

[Carex fraseri Andrews; Cymophyllus fraseri (Andrews) Mack.; C. fraserianus (Ker Gawl.) Kartesz \& Gandhi]

Carex gigantea Rudge, Giant Sedge, S3/G4 (Naczi 8948 DOV)

[Carex grandis L. H. Bailey]

Carex glaucescens Elliott, Southern Waxy Sedge, S1/G4 (McAvoy 4556 DOV)
Carex glaucodea Tuck. ex Olney, Blue Sedge (Longbottom 4151 DOV)

[Carex flaccosperma Dewey var. glaucodea (Tuck. ex Olney) Kük.]

Carex gracilescens Steud., Slender Sedge (Naczi 11048 DOV)

Carex gracillima Schwein., Graceful Sedge (McAvoy 4405 DOV)

Carex granularis Muhl. ex Willd., Limestone Meadow Sedge (McAvoy 4355 DOV)

Carex gravida L. H. Bailey, Heavy Sedge, † (McAvoy 3589 DOV)

Carex grayi J. Carey, Gray's Sedge (Naczi 8056 DOV)

Carex grisea Wahlenb., Inflated Narrow-leaf Sedge (McAvoy 2225 DOV)

[Carex amphibola Steud. var. turgida Fernald]

Carex gynandra Schwein., Nodding Sedge (Lea 3201 DOV)

Carex haydenii Dewey, Hayden's Sedge, S1/G5 (Knapp 1392 DOV)

Carex hirsutella Mack., Fuzzy Wuzzy Sedge (Lea 2761 DOV) [Carex hirsuta Willd.; C. triceps auct. non Michx.]

Carex hirta L., Hammer Sedge, † (Berdine s.n. TAWES)

Carex birtifolia Mack., Hairy Sedge, S3/G5 (Lea 3150 DOV)

Carex hitchcockiana Dewey, Hitchcock's Sedge, S1/G5 (Ebert \& Holt 322EH)

Carex hormathodes Fernald, Marsh Straw Sedge (Hill 13948 Assateague Island National Seashore Herbarium)

Carex hyalinolepis Steud., Shoreline Sedge, S2S3/G4G5 (Lea 2637 DOV)

Carex hystericina Muhl. ex Willd., Bottlebrush Sedge, S1/G5 (McAvoy 1097 DOV)

Carex interior L. H. Bailey, Inland Sedge, S1/G5 (Frye \& Harrison s.n. DOV)

Carex intumescens Rudge, Greater Bladder Sedge (Longbottom 7514 DOV)

[Carex intumescens var. fernaldii L. H. Bailey]

Carex jamesii Schwein., James' Sedge (Naczi 8051 DOV)

Carex joorii L. H. Bailey, Cypress Swamp Sedge, S3/G4G5 (Longbottom \& Knapp 6507 DOV)

Carex kobomugi Ohwi, Japanese Sedge, † (Lea 1290 Assateague Island National Seashore Herbarium)

Carex kraliana Naczi \& Bryson, Kral's Sedge (Knapp 1180 DOV)

Carex lacustris Willd., Lake Sedge, S2/G5 (McAvoy 1142 DOV)

Carex laevivaginata (Kük.) Mack., Smoothsheath Sedge (Longbottom 5938 DOV)

Carex lasiocarpa Ehrh., Woolyfruit Sedge, S1/G5 (Knapp et al. $1400 \mathrm{DOV})$

[Carex lasiocarpa var. americana Fernald]

Carex laxiculmis Schwein. var. copulata L. H. Bailey, Spreading Sedge, S1?/G5T3T5 (Naczi 11055 NY)

Carex laxiculmis Schwein. var. laxiculmis, Lax-culmed Sedge (Naczi $8058 \mathrm{NY}$ ) 
Carex laxiflora Lam., Broad Loose-flowered Sedge (Naczi 9321 $\mathrm{NY})$

[Carex laxiflora var. patulifolia (Dewey) J. Carey; C. laxiflora var. serrulata F. J. Herm.]

Carex leavenworthii Dewey, Leavenworth's Sedge (Longbottom 11374 DOV)

Carex leptalea Wahlenb. var. harperi (Fernald) Stone, Harper's Bristly-stalked Sedge (Lea 2616 DOV)

Carex leptalea Wahlenb. var. leptalea, Bristly-stalked Sedge (Knapp et al. 1400a DOV)

Carex leptonervia (Fernald) Fernald, Nerveless Woodland Sedge (Naczi $11056 \mathrm{NY)}$

Carex lonchocarpa Willd. ex Spreng., Southern Long Sedge (Longbottom 5870 DOV)

[Carex folliculata L. var. australis L. H. Bailey; C. smalliana Mack.]

Carex longii Mack., Long's Sedge (Hill 15736 Assateague Island National Seashore Herbarium)

Carex louisianica L. H. Bailey, Louisiana Sedge, S3/G5 (Naczi 11579 DOV)

Carex lucorum Willd. ex Link, Blue Ridge Sedge, S1/G4 (Ebert \& Holt s.n. DOV)

Carex lupuliformis Sartwell ex Dewey, False Hop Sedge, S2/ G4 (Knapp 656 DOV)

Carex lupulina Muhl. ex Willd., Hop Sedge (Knapp \& Frye $656 a \mathrm{DOV})$

Carex lurida Wahlenb., Shallow Sedge (Hill 557 Assateague Island National Seashore Herbarium)

Carex meadii Dewey, Mead's Sedge, S1/G4G5 (Frye 5537 TAWES)

Carex mesochorea Mack., Midland Sedge (Longbottom et al. 7202 DOV)

Carex michauxiana Boeckeler, Michaux's Sedge, SH/G5 (Riefner 81-109 MICH)

Carex mitchelliana M. A. Curtis, Mitchell's Sedge, S2/G4 (Longbottom 6139 DOV)

Carex molesta Mack. ex Bright, Troublesome Sedge, †? (Knapp 2848 DOV)

Carex molestiformis Reznicek \& P. Rothrock, Frightfruit Sedge, $\dagger$ (Frye $6178 \mathrm{MICH})$

Carex mublenbergii Schkuhr ex Willd var. enervis Boott, Muhlenberg's Nerveless Sedge (Holt \& Ebert s.n. TAWES)

[Carex mublenbergii var. xalapensis (Kunth) Britton; C. plana Mack.]

Carex mublenbergii Schkuhr ex Willd. var. mublenbergii, Muhlenberg's Sedge (Lea 2773 DOV)

Carex muricata L., Rough Sedge, † (Baltars 4414 Cylburn Arboretum) - the specimen vouchering this report could not be examined for this project, but Frye and Lea (2001) examined the specimen for their study

[Carex pairaei F. W. Schultz]

Carex nigromarginata Schwein., Black Edge Sedge (Knapp 2386 DOV)

Carex normalis Mack., Greater Straw Sedge (Naczi 8049 DOV)
- Carex novae-angliae Schwein., New England Sedge-see Excluded Flora section.

Carex oklahomensis Mack., Oklahoma Sedge, † (Longbottom 11694 NY)

Carex oligocarpa Willd., Rich Woods Sedge (Naczi 11051 NY)

Carex oxylepis Torr. \& Hook., Sharp Scale Sedge, S1?/G5 (Thomson 970515 TAWES)

- Carex pallescens L., Pale Sedge-see Excluded Flora section.

Carex pedunculata Muhl. ex Willd., Longstalk Sedge, S1/G5 (Longbottom et al. 6029 DOV)

Carex pellita Muhl. ex Willd., Wooly Sedge, S2?/G5 (Naczi 9274 DOV)

[Carex lanuginosa Michx.]

Carex pensylvanica Lam., Pennsylvania Sedge (Knapp 1170 DOV)

Carex planispicata Naczi, Flat-spiked Sedge, S1S2/G4 (Naczi $8060 \mathrm{NY})$

[Carex amphibola Steud. var. rigida (L. H. Bailey) Fernald]

Carex plantaginea Lam., Plantain-feaf Sedge, S1?/G5 (Naczi $11054 \mathrm{NY}$ )

Carex platyphylla J. Carey, Broadleaf Sedge (McAvoy 5778 DOV)

Carex polymorpha Muhl., Variable Sedge, SH/G3 (Crawford s.n. $\mathrm{PH}$

Carex prasina Wahlenb., Drooping Sedge (Naczi 9328c DOV)

Carex projecta Mack., Necklace Sedge, S2/G5 (Lea 3148 DOV)

Carex radiata (Wahlenb.) Small, Eastern Star Sedge (Naczi 8043 DOV)

[Carex rosea Schkuhr ex Willd. var. radiata (Wahlenb.) Dewey]

Carex retroflexa Muhl. ex Schkuhr, Reflexed Sedge (McAvoy 4631 DOV)

- Carex retrorsa Schwein., Knotsheath Sedge-see Excluded Flora section.

Carex reznicekii Werier, Reznicek's Sedge (Knapp 2394 DOV)

Carex richardsonii R. Br., Richardson's Sedge, S1/G4 (Knapp 1149 DOV)

Carex rosea Schkuhr ex Willd., Rosy Sedge (Knapp 2847 DOV) [Carex convoluta Mack.]

- Carex rostrata Stokes, Beaked Sedge-see Excluded Flora section.

- Carex sartwellii Dewey, Sartwell's Sedge-see Excluded Flora section.

Carex scabrata Schwein., Eastern Rough Sedge (Knapp 03-02 TAWES)

Carex scoparia Schkuhr, Pointed Broom Sedge (Naczi 8045 DOV)

Carex seorsa Howe, Weak Stellate Sedge (Knapp et al. 1807 DOV)

Carex shortiana Dewey, Short's Sedge, S3S4/G5 (Longbottom et al. $5995 \mathrm{DOV})$

Carex silicea Olney, Beach Sedge, S1/G5 (Hill 13929 Assateague Island National Seashore Herbarium) 
Carex sparganioides Muhl. ex Willd., Bur-reed Sedge, S3/G5 (Naczi 8055 DOV)

Carex spicata Huds., Prickly Sedge, † (Tatnall 1144 DOV)

Carex squarrosa L., Squarrose Sedge (Naczi 8041 DOV)

Carex stipata Muhl. ex Willd. var. maxima Chapm., Stalkgrain Sedge (Lea 2000 DOV)

[Carex stipata var. uberior C. Mohr; C. uberior (C. Mohr) Mack.]

Carex stipata Muhl. ex Willd. var. stipata, Owlfruit Sedge (Naczi 8042 DOV)

Carex straminea Willd., Eastern Straw Sedge S1S2/G5 (Lea 2843 DOV)

Carex striata Michx., Walter's Sedge (Longbottom \& Knapp 6284 DOV)

[Carex walteriana L. H. Bailey]

Carex striatula Michx., Lined Sedge, S3/G5 (Knapp \& Frye 1376 DOV)

Carex stricta Lam., Tussock Sedge (Naczi 8046 DOV)

[Carex strictior Dewey]

Carex styloflexa Buckley, Bent Sedge (Naczi 11335 NY)

Carex swanii (Fernald) Mack., Swan's Sedge (Naczi 8059 DOV)

Carex tenera Dewey, Quill Sedge, SH/G5 (Leonard 19864 US)

- Carex tenuiflora Wahlenb., Sparseflowered Sedge-see Excluded Flora section.

Carex tetanica Schkuhr var. canbyi Porter, Rigid Sedge, - S1/G4G5T1T2Q (Knapp 4518 NY)

Carex texensis (Torr.) L. H. Bailey, Texas Sedge, † (Naczi 11336 DOV)

Carex tonsa (Fernald) E. P. Bicknell var. tonsa, Shaved Sedge (Knapp et al. 1808 DOV)

Carex tonsa (Fernald) E. P. Bicknell var. rugosperma (Mack.) Crins, Parachute Sedge (Frye \& Lea s.n. TAWES)

Carex torta Boott ex Tuck., Twisted Sedge (Knapp 2714 DOV)

Carex tribuloides Wahlenb. var. tribuloides, Blunt Broom Sedge (Longbottom 6155 DOV)

Carex trichocarpa Willd., Hairy-fruited Sedge, S2/G4 (Lea 1518 TAWES)

Carex trisperma Dewey, Three-seeded Sedge (Lea 2716 DOV)

Carex tuckermanii Dewey, Tuckerman's Sedge, S1/G4 (Lea 2740 DOV)

Carex typhina Michx., Cattail Sedge (Longbottom 11722 DOV)

Carex umbellata Schkuhr ex Willd., Parasol Sedge (Knapp 2380 DOV)

Carex utriculata Boott, Swollen-beaked Sedge (Lea 2741 TAWES)

[Carex rostrata Stokes var. utriculata (Boott) L. H. Bailey]

Carex venusta Dewey, Darkgreen Sedge, S3S4/G4 (Naczi 11564 DOV)

[Carex oblita Steud.; C. venusta var. minor Boeckler]

- Carex verrucosa Muhl., Warty Sedge-see Excluded Flora section.

Carex vesicaria L., Lesser Bladder Sedge, S1/G5 (Sipple 2020 DOV)

Carex vestita Willd., Velvet Sedge, S2/G5 (Naczi 9298 DOV)
Carex virescens Muhl. ex Willd., Ribbed Sedge (Knapp 2845 DOV)

- Carex viridula Michx., Little Green Sedge-see Excluded Flora section.

Carex vulpinoidea Michx., Fox Sedge (Longbottom 11609 DOV)

Carex willdenowii Schkuhr ex Willd., Willdenow's Sedge (Naczi 11052 DOV)

Carex woodii Dewey, Pretty Sedge (Naczi 11049 DOV)

Cladium mariscoides (Muhl.) Torr., Smooth Sawgrass (Naczi 10762 DOV)

[Mariscus mariscoides (Muhl.) Kuntze]

Cyperus acuminatus Torr. \& Hook., Taper-tipped Flatsedge (Davis 7228 Patuxent River Naval Air Station Herbarium)

Cyperus bipartitus Torr., Slender Flatsedge (McAvoy 6686 DOV) [Cyperus rivularis Kunth]

Cyperus brevifolioides Thieret \& Delahouss., Asiatic Headsedge, † (McAvoy $6710 \mathrm{DOV})$

[Kyllinga gracillima Miq.]

Cyperus compressus L., Annual Flatsedge, †? (Canby s.n. DOV)

Cyperus croceus Vahl, Baldwin's Flatsedge (Harms 6877 US) [Cyperus globulosus Aubl.; C. multiflorus (Britton) Small; C. retrorsus Chapm. var. robustus (Boeckler) Kük.]

Cyperus dentatus Torr., Toothed Flatsedge, SH/G4 (Tatnall 1922 DOV)

Cyperus diandrus Torr., Umbrella Flatsedge, SU/G5 (McAvoy 1815 DOV)

Cyperus difformis L., Smallflower Umbrellasedge, $\ddagger$ (Longbottom $12091 \mathrm{NY}$ )

Cyperus echinatus (L.) Alph. Wood, Globe Flatsedge (Hill 15861 Assateague Island National Seashore Herbarium) [Cyperus ovularis (Michx.) Torr.]

- Cyperus engelmannii Steud., Engelman's Flatsedge-see Excluded Flora section.

Cyperus erythrorhizos Muhl., Redroot Flatsedge (McAvoy 4515 DOV) [Cyperus halei Torr. ex Britton]

Cyperus esculentus L. var. leptostachyus Boeckler, Yellow Nutsedge, ‡? (Longbottom 6876 DOV) [Cyperus esculentus var. sativus Boeckler]

Cyperus filicinus Vahl, Fern Flatsedge (Longbottom 14304 NY) [Cyperus nuttallii Eddy ex Spreng.]

Cyperus filiculmis Vahl, Southeastern Flatsedge (Naczi 11569 o Knapp NY)

Cyperus flavescens L., Yellow Flatsedge (Hill 15516 Assateague Island National Seashore Herbarium)

Cyperus flavicomus Michx., White-edge Flatsedge, † (Longbottom $10451 \mathrm{NY}$ )

Cyperus fuscus L., Brown Flatsedge, $†$ (Plitt s.n. US)

Cyperus grayi Torr., Gray's Flatsedge (Naczi 11568 DOV)

Cyperus haspan L., Haspan Flatsedge, S1?/G5 (Hirst s.n. DOV) 
Cyperus hortensis (Slazm. ex Steud.) Dorr, Annual Hedgesedge (Strong et al. 1627 US)

[Cyperus tenuifolius (Steud.) Dandy; Kyllinga pumila Michx.]

Cyperus houghtonii Torr., Houghton's Flatsedge, S1/G4? (Riefner 80/293 MARY)

Cyperus bystricinus Fernald, Bristly Flatsedge, S2/G4 (Naczi 11573 DOV)

Cyperus iria L., Rice Flatsedge, † (Longbottom 6914 DOV)

Cyperus lancastriensis Porter, Many-flowered Flatsedge, S2S3/G5 (Longbottom $19760 \mathrm{NY)}$

Cyperus lupulinus (Spreng.) Marcks ssp. lupulinus, Great Plains Flatsedge (McAvoy 4455 DOV)

Cyperus lupulinus (Spreng.) Marcks ssp. macilentus (Fernald) Marcks, Meager Flatsedge (Shull 272 US)

Cyperus microiria Steud., Asian Flatsedge, † (Longbottom $12007 \mathrm{NY}$ )

Cyperus odoratus L., Corky Flatsedge (Hill 15662 Assateague Island National Seashore Herbarium)

Cyperus plukenetii Fernald, Plukenet's Flatsedge, SH/G5 (Tatnall 4325 DOV)

Cyperus polystachyos Rottb., Many-spike Flatsedge (Hirst \& Wilson s.n. DOV)

[Cyperus microdontus Torr.; C. paniculatus Rottb.; C. polystachyos var. texensis (Torr.) Fernald]

Cyperus pseudovegetus Steud., Marsh Flatsedge (McAvoy 4479 DOV)

Cyperus refractus Engelm. ex Boeckler, Reflexed Flatsedge, S2?/G5 (McAvoy 6637 DOV)

Cyperus retrofractus (L.) Torr., Rough Flatsedge, S2/G5 (McAvoy 6638 DOV)

[Cyperus dipsaciformis Fernald]

Cyperus retrorsus Chapm., Pine Barrens Flatsedge (Naczi 11567 DOV)

[Cyperus cylindricus (Elliott) Britton; C. nashii Britton; C. retrorsus var. cylindricus (Elliott) Fernald; C. retrorsus var. nashii (Britton) Fernald \& Griscom; C. torreyi Britton]

- Cyperus rotundus L., Purple Nutsedge, † Waif Reed (1964)

Cyperus squarrosus L., Bearded Flatsedge (Hill 15473 Assateague Island National Seashore Herbarium) [Cyperus aristatus Rottb.; C. inflexus Muhl.]

Cyperus strigosus L., False Nutsedge (McAvoy 4606 DOV) [Cyperus strigosus var. robustior Britton; C. stenolepis Torr.]

Cyperus subsquarrosus (Muhl.) Bauters, Small-flowered Dwarfbulrush, S1/G5 (Hicks s.n. US)

[Hemicarpha micrantha (Vahl) Pax; Lipocarpha micrantha (Vahl) G. C. Tucker]

Dulichium arundinaceum (L.) Britton, Three-way Sedge (Naczi 8951 DOV)

Eleocharis acicularis (L.) Roem. \& Schult., Needle Spikerush (Strong et al. 1759 US)
Eleocharis albida Torr., White Spikerush S2S3/G4G5 (Knapp 957 TAWES)

Eleocharis ambigens Fernald, Creeping Spikerush (Longbottom $7453 \mathrm{NY}$ )

Eleocharis compressa Sull. var. compressa, Flatstem Spikerush, - S1/G4 (Donnell s.n. US)

Eleocharis engelmannii Steud., Engelmann's Spikerush (Hotchkiss 7847 US)

Eleocharis equisetoides (Elliott) Torr., Jointed Spikerush, S1/ G4 (Otis s.n. DOV)

Eleocharis erythropoda Steud., Bald Spikerush, SU/G5 (Knapp 2608 TAWES)

[Eleocharis calva Torr.]

- Eleocharis fallax Weath., Creeping Spikerush-see Excluded Flora section.

- Eleocharis flavescens (Poir.) Urb., Yellow Spikerush-see Excluded Flora section.

- Eleocharis geniculata (L.) Roem. \& Schult., Canada Spikerush-see Excluded Flora Section.

Eleocharis halophila (Fernald \& Brackett) Fernald, Saltmarsh Spikerush, S1/G4Q (Lea 2800 TAWES)

Eleocharis intermedia Schult., Mudflat Spikerush, S1/G5 (Stieber 111 US)

Eleocharis melanocarpa Torr., Blackfruit Spikerush, S1/G4 (Knapp 569 DOV)

Eleocharis microcarpa Torr. var. filiculmis Torr., Small-fruited Spikerush (Knapp 565 DOV)

[Eleocharis torreyana Boeckler]

Eleocharis obtusa (Willd.) Schult., Blunt Spikerush (Naczi 8989 DOV)

Eleocharis olivacea Torr., Bright Green Spikerush (Knapp 571 DOV)

[Eleocharis flaccida (Rchb.) Urb.; E. flavescens (Poir.) Urb. var. olivacea (Torr.) Gleason; E. ochreata Nees]

- Eleocharis ovata Torr., Ovata Spikerush-see Excluded Flora section.

Eleocharis palustris (L.) Roem. \& Schult., Common Spikerush (Frye 6219 TAWES)

[Eleocharis palustris var. major Sond.; E. smallii Britton]

Eleocharis parvula (Roem. \& Schult.) Link, Dwarf Spikerush (Strong et al. 1170 US) [Scirpus nanus Spreng.]

Eleocharis quadrangulata (Michx.) Roem. \& Schult., Squarestem Spikerush (Knapp 584 DOV)

Eleocharis robbinsii Oakes, Robbins' Spikerush, S1/G4G5 (Knapp 1591 DOV)

Eleocharis rostellata (Torr.) Torr., Beaked Spikerush, S2?/G5 (Hotchkiss 7324 US)

Eleocharis tenuis (Willd.) Schult. var. pseudoptera (Weath.) Svenson, False-winged Slender Spikerush (Thompson 514 MARY)

[Eleocharis capitata (L.) R. Br. var. pseudoptera Weath.] 
Eleocharis tenuis (Willd.) Schult. var. tenuis, Slender Spikerush (Dietrich 9 MARY)

Eleocharis tenuis (Willd.) Schult. var. verrucosa (Svenson) Svenson, Warty Slender Spikerush (Norton 2184 MARY) [Eleocharis capitata (L.) R. Br. var. verrucosa Svenson]

Eleocharis tortilis (Link) Schult., Twisted Spikerush, S3/G5 (Strong 888 US)

Eleocharis tricostata Torr., Three-angled Spikerush, S1/G4 (Knapp 541 TAWES)

Eleocharis tuberculosa (Michx.) Roem. \& Schult., Long-tubercled Spikerush (Knapp 563 TAWES)

[Eleocharis simplex (Elliott) A. Dietr.]

Eriophorum gracile WD. J. Koch ex Roth, Slender Cottongrass, - S1/G5 (Frye 1088 \& Berdine TAWES)

Eriophorum virginicum L., Tawny Cottongrass, S3/G5 (Hitchcock 1568 US)

Fimbristylis annua (All.) Roem. \& Schult., Annual Fimbry, - S3/G4G5 (Tidestrom \& Bartlett 5194 US)

[Fimbristylis baldwiniana (Schult.) Torr.]

Fimbristylis autumnalis (L.) Roem. \& Schult., Slender Fimbry (Strong et al. 1758 US)

[Fimbristylis geminata (Nees) Kunth]

Fimbristylis caroliniana (Lam.) Fernald, Carolina Fimbry, - S1S2/G4 (Hill 15507 Assateague Island National Seashore Herbarium)

[Fimbristylis harperi Britton]

Fimbristylis castanea (Michx.) Vahl, Marsh Fimbry (Fassett 22369 US)

Fimbristylis perpusilla Harper ex Small \& Britton, Harpers Fimbry, S2/G2 (Berdine AB95-149 TAWES)

- Fimbristylis puberula (Michx.) Vahl var. puberula, Hairy Fimbry, SU/G5-see Excluded Flora Section.

[Fimbristylis spadicea (L.) Vahl]

Fuirena pumila (Torr.) Spreng., Dwarf Umbrellasedge, - S2S3/G4 (Hill 1572 Assateague Island National Seashore Herbarium)

Fuirena squarrosa Michx., Hairy Umbrellasedge (Naczi 10754 DOV)

[Fuirena squarrosa var. hispida (Elliott) Chapm.; F. hispida Elliott]

Rhynchospora alba (L.) Vahl, White Beaksedge, S3/G5 (Knapp 1644 TAWES)

-Rhynchospora capillacea Torr., Needle Beaksedge—see Excluded Flora section.

Rhynchospora capitellata (Michx.) Vahl, Brownish Beaksedge (Naczi 8867 DOV)

Rhynchospora cephalantha A. Gray, Bunched Beaksedge, - S1/G5 (Knapp 1107 TAWES) [Rhynchospora axillaris (Lam.) Britton]

Rhynchospora chalarocephala Fernald \& Gale, Loose-headed Beaksedge(Knapp 597 TAWES)
Rhynchospora corniculata (Lam.) A. Gray, Short-bristled Horned Beaksedge(Wiegand s.n. TAWES)

- Rhynchospora filifolia A. Gray, Threadleaf Beaksedge-see Excluded Flora section.

Rhynchospora fusca (L.) Aiton, Brown Beaksedge, SH/G5 (Riefner 80-262 MICH)

Rhynchospora globularis (Chapm.) Small var. globularis, Globe Beaksedge, S1/G5T5? (Sipple $1704 \mathrm{PH}$ )

[Rhynchospora cymosa Elliott var. globularis Chapm.]

Rhynchospora glomerata (L.) Vahl, Clustered Beaksedge, S3/G5 (Knapp 1055 TAWES)

[Rhynchospora cymosa Elliott; R. glomerata var. paniculata Chapm.]

Rhynchospora gracilenta A. Gray, Slender Beaksedge (Knapp 82-02 TAWES)

Rhynchospora harperi Small, Harper's Beaksedge, S1/G4? (Knapp 84-02 TAWES)

Rhynchospora inexpansa (Michx.) Vahl, Nodding Beaksedge (Knapp 641 \& Frye TAWES)

Rhynchospora inundata (Oakes) Fernald, Narrow-fruited Horned Beaksedge, S1/G4? (Knapp 1674 TAWES)

Rhynchospora macrostachya Torr. ex A. Gray, Tall Horned Beaksedge (Naczi $8864 \mathrm{NY}$ )

Rhynchospora microcephala (Britton) Britton, Small-headed Beaksedge, S2/G5 (Frye 700 TAWES)

[Rhynchospora cephalantha A. Gray var. microcephala (Britton) Kük.]

Rhynchospora nitens (Vahl) A. Gray, Short-beaked Beaksedge, - S1/G4? (Frye et al. 5322 TAWES) [Psilocarya nitens (Vahl) Alph. Wood]

Rhynchospora oligantha A. Gray, Feather-bristle Beaksedge, - SH/G4 (Sargent 6370 US)

Rhynchospora pallida M. A. Curtis, Pale Beaksedge, SH/G3 (Canby s.n. US)

Rhynchospora rariflora (Michx.) Elliott, Few-flowered Beaksedge, SU/G5 (Knapp 2324 US)

Rhynchospora recognita (Gale) Kral, Round-headed Beaksedge, S2/G5T5? (Knapp 1541 TAWES)

[Rhynchospora globularis (Chapm.) Small var. recognita Gale]

Rhynchospora scirpoides (Torr.) A. Gray, Long-beaked Beaksedge, S2/G4 (Knapp et al. 74-02 TAWES) [Psilocarya scirpoides Torr.]

Rhynchospora torreyana A. Gray, Torrey's Beaksedge, S2/G4 (Knapp 597 DOV)

Schoenoplectiella mucronata (L.) J. Jung \& H. K. Choi, Ricefield Bullrush, † (Knapp 2107 DOV)

[Schoenoplectus mucronatus (L.) Palla]

Schoenoplectiella purshiana (Fernald) Lye var. purshiana, Weak-stalked Bulrush (Strong et al. 1762 US)

[Scirpus debilis Pursh; S. purshianus Fernald; Schoenoplectus purshianus (Fernald) M. T. Strong] 
Schoenoplectiella purshiana (Fernald) Lye var. williamsii (Fernald) Hayas., Williams' Weak-stalked Bulrush (Simmons $3792 \mathrm{NY}$ )

Schoenoplectiella smithii (A. Gray) Hayas. var. setosa (Fernald) Hayas., Smith's Bulrush, S1?/G5? (Longbottom 12362 NY) [Schoenoplectus smithii (A. Gray) Soják var. setosus (Fernald) S. G. Sm.]

- Schoenoplectus acutus (Muhl. ex Bigelow) Á. Löve \& D. Löve var. acutus, Hardstem Bulrush-see Excluded Flora section.

Schoenoplectus americanus (Pers.) Volkart ex Schinz \& R. Keller, Chair-makers Bulrush (Fosberg 34811 US)

[Scirpus americanus Pers.; S. olneyi A. Gray]

Schoenoplectus etuberculatus (Steud.) Soják, Canby's Bulrush, - SH/G3G4 (Stasz s.n. DOV)

[Scirpus etuberculatus (Steud.) Kunze]

Schoenoplectus pungens (Vahl) Palla var. pungens, Common Three-square (Fosberg 42431 US)

[Scirpus pungens Vahl]

Schoenoplectus subterminalis (Torr.) Soják, Swaying Bulrush, - S1/G4G5 (Benedict et al. 6286 US)

[Scirpus subterminalis Torr.]

Schoenoplectus tabernaemontani (C. C. Gmel.) Palla, Softstem Bulrush (Longbottom 3081 US)

[Scirpus lacustris L.; S. tabernaemontani C. C. Gmel.; S. validus Vahl]

Schoenoplectus torreyi (Olney) Palla, Torrey's Bulrush, S1/ G5? (Knapp 4518 TAWES)

Scirpus ancistrochaetus Schuyler, Northeastern Bulrush, S1/ G3, LE (Bartgis $1290 \mathrm{PH}$ )

Scirpus atrovirens Willd., Green Bulrush (Hitchcock 15637 US)

Scirpus cyperinus (L.) Kunth, Wool Grass (Hotchkiss 6027 US)

[Scirpus cyperinus var. eriophorum (Michx.) Kunth; S. cyperinus var. pelius Fernald; S. eriophorum Michx.; S. rubricosus Fernald]

Scirpus expansus Fernald, Woodland Bulrush, S3/G4 (Lea 3218 US)

[Scirpus sylvaticus L.]

Scirpus georgianus R. M. Harper, Georgia Bulrush (Kanal 612 US)

[Scirpus atrovirens Willd. var. georgianus (R. M. Harper) Fernald]

Scirpus hattorianus Makino, Mosquito Bulrush, SU/G5 (Schuyler $4881 \mathrm{PH})$

- Scirpus lineatus Michx., Drooping Bulrush-see Excluded Flora section.

Scirpus pendulus Muhl., Nodding Bulrush (Baltars 188 US)

Scirpus polyphyllus Vahl, Leafy Bulrush (Leonard 21419 US)

Scleria minor (Britton) W. Stone, Slender Nutrush, S1/G4 (Knapp 48-02 TAWES)

[Scleria triglomerata Michx. var. minor Britton]
Scleria mublenbergii Steud., Muhlenberg's Nutrush, S1S2/G5 (Boone s.n. TAWES)

[Scleria setacea Poir.]

Scleria oligantha Michx., Little-headed Nutrush, S1/G5 (Knapp et al. 4295 TAWES)

Scleria pauciflora Muhl. ex Willd., Few-flowered Nutrush, - S3/G5 (Knapp \& McAvoy 3908 MARY)

[Scleria pauciflora var. caroliniana Alph. Wood]

Scleria reticularis Michx., Netted Nutrush, S2S3/G4 (McAvoy 1287 DOV)

[Scleria torreyana Walp.]

Scleria triglomerata Michx., Whip Nutrush, S3 (Knapp 595 DOV)

[Scleria nitida Fernald, S1/GNR]

Scleria verticillata Muhl. ex Willd., Low Nutrush, S1/G5 (Hill \& Boone 15752 Assateague Island National Seashore Herbarium)

Trichophorum planifolium (Spreng.) Palla, Bashful Bulrush, - S2/G4G5 (Frye 5904 TAWES)

[Scirpus verecundus Fernald]

DIAPENSIACEAE

(Diapensia Family)

Galax urceolata (Poir.) Brummitt, Galax, SX/G5 (Sherard s.n. OXF)—not seen since the colonial era; see Brown et al. (1987).

\section{DIOSCOREACEAE}

(Yam Family)

Dioscorea polystachya Turcz., Chinese Yam, $\ddagger$ (Riefner 76-563 BALT)

[Dioscorea batatas Decne.; D. oppositifolia L.]

Dioscorea villosa L., Wild Yam (Ditman s.n. MARY)

[Dioscorea glauca Muhl. ex Bartlett; D. hirticaulis Bartlett; D. quaternata J. F. Gmel.; D. villosa var. glabrifolia (Bartlett) Fernald; D. villosa var. hirticaulis (Bartlett) H. E. Ahles]

DROSERACEAE

(Sundew Family)

Drosera brevifolia Pursh, Dwarf Sundew (McAvoy et al. 7843 DOV)

Drosera capillaris Poir., Pink Sundew, S1/G5 (McAvoy 1145 DOV)

Drosera filiformis Raf., Threadleaf Sundew, † (Strong 2639 US)

Drosera intermedia Hayne, Spoonleaf Sundew (Walker 4159 US)

Drosera rotundifolia L., Roundleaf Sundew, S3/G5 (Strong \& Sheridan 1145 US)

EBENACEAE

(Ebony Family)

Diospyros virginiana L., Persimmon (Stickel s.n. US) 


\section{ELAEAGNACEAE}

(Oleaster Family)

Elaeagnus angustifolia L., Russian Olive, † (Longbottom 10936 DOV)

Elaeagnus commutata Bernh. ex Rydb., American Silverberry, † (Brisbane 24 MARY)

- Elaeagnus multiflora Thunb., Cherry Silverberry-see Excluded Flora section.

Elaeagnus pungens Thunb., Thorny Olive, † (Longbottom $27655 \mathrm{NY}$ )

Elaeagnus umbellata Thunb., Autumn Olive, $\ddagger$ (Ubler s.n. US)

\section{ELATINACEAE}

(Waterwort Family)

Elatine americana (Pursh) Arn., American Waterwort, SU/G4 (Naczi 588 \& Outten DOV)

[Elatine triandra Schkuhr var. americana (Pursh) Fassett]

Elatine minima (Nutt.) Fisher \& C. A. Mey., Small Waterwort, S1?/G5 (Tatnall 3958 DOV)

Elatine triandra Schkuhr, Three-stamen Waterwort (Metheny 0006 MARY)

\section{ERICACEAE}

(Heath Family)

Arctostaphylos uva-ursi (L.) Spreng., Bearberry, S1/G5 (Frye s.n. TAWES)

Chamaedaphne calyculata (L.) Moench, Leatherleaf, S1/G5 (Williams 591 DOV)

Chimaphila maculata (L.) Pursh, Striped Wintergreen (McAvoy 2078 DOV)

Chimaphila umbellata (L.) W. P. C. Barton, Common Wintergreen, S3/G5T5 (Sipple 2045 DOV)

[Chimaphila umbellata var. cisatlantica S. F. Blake]

Epigaea repens L., Trailing Arbutus (Naczi 8093 DOV)

Eubotrys racemosa (L.) Nutt., Fetterbush (Boone s.n. TAWES)

[Leucothoe racemosa (L.) A. Gray]

Gaultheria bispidula (L.) Muhl. ex Bigelow, Creeping Snowberry, S1/G5 (Thompson \& Feller s.n. TAWES)

Gaultheria procumbens L., Eastern Teaberry (Tatnall 3119 DOV)

Gaylussacia baccata (Wangenh.) K. Koch, Black Huckleberry (Boone s.n. TAWES)

[Decachaena baccata (Wangenh.) Small]

Gaylussacia brachycera (Michx.) A. Gray, Box Huckleberry, - S1/G3 (Tidestrom 12018 US)

[Buxella brachycera (Michx.) Small]

Gaylussacia dumosa (S. Andrews) Torr. \& A. Gray, Dwarf Huckleberry, S1/G5 (Walker 111 MARY)

[Gaylussacia bigeloviana (Fernald) Sorrie \& Weakley;

G. dumosa var. bigeloviana Fernald; Lasiococcus dumosus

(S. Andrews) Small]
Gaylussacia frondosa (L.) Torr. \& A. Gray ex Torr., Dangleberry (Knapp 1169 DOV)

[Decachaena frondosa (L.) Torr. \& A. Gray]

Kalmia angustifolia L., Sheep Laurel, S3S4/G5 (Knapp \& Frye 97-02 TAWES)

Kalmia latifolia L., Mountain Laurel (McAvoy 2079 DOV)

Leucothoe axillaris (Lam.) D. Don, Coastal Doghobble, † (Knapp 1137 \& Frye TAWES)

[Leucothoe catesbaei (Walter) A. Gray]

Leucothoe fontanesiana (Steud.) Sleumer, Highland Doghobble, $\dagger($ Redman 7023 BALT)

[Leucothoe axillaris (Lam.) D. Don var. editorum (Fernald \& G. B. Schub.) H. E. Ahles; L. walteri (Willd.) Melvin]

Lyonia ligustrina (L.) DC., Maleberry (Knapp 31-03 TAWES) [Arsenococcus frondosus (Pursh) Small; A. ligustrinus (L.) Small; Lyonia ligustrina var. foliosiflora (Michx.) Fernald]

Lyonia mariana (L.) D. Don, Staggerbush (Knapp 1333 DOV)

Menziesia pilosa (Michx.) Juss., Minniebush (Knapp 990 DOV) [Rhododendron pilosum (Michx.) Craven]

Monotropa hypopitys L., American Pinesap (Longbottom et al. 5041 DOV)

[Hypopitys americana (DC.) Small; H. hypopitys (L.) Small; H. lanuginosa (Michx.) Nutt.]

Monotropa uniflora L., Indian Pipe (Redmond 244 DOV)

[Monotropa brittonii Small]

Monotropsis odorata Schwein. ex Elliott, Sweet Pinesap, S1/ G3 (Freeman s.n. US)

Orthilia secunda (L.) House, Sidebells Wintergreen, SH/G5 (Fosberg19026 US)

[Pyrola secunda L.]

Oxydendrum arboreum (L.) DC., Sourwood, S1/G5 (Steury 000824.7 US)

Pieris floribunda (Pursh) Benth. \& Hook. f., Mountain Fetterbush, †? (Simmons et al. 3064 TAWES)

Pyrola americana Sweet, American Wintergreen (McAvoy 818 DOV)

[Pyrola rotundifolia L. var. americana (Sweet) Fernald]

Pyrola chlorantha Sw., Green-flowered Wintergreen, SH/G5 (Fosberg 19019 US)

[Pyrola virens var. convoluta (Barton) Fernald; P. virens Schreb. var. virens]

Pyrola elliptica Nutt., Shinleaf (Hotchkiss 5789 US)

Rhododendron arborescens (Pursh) Torr., Smooth Azalea, S3/ G4G5 (Schrock s.n. NCU)

[Azalea arborescens Pursh] 
Rhododendron atlanticum (Ashe) Rehder, Dwarf Azalea (Henry s.n. $\mathrm{GH})$

[Azalea atlantica Ashe]

- Rbododendron calendulaceum (Michx.) Torr., Flame Azaleasee Excluded Flora section.

[Azalea calendulacea Michx.]

- Rhododendron canescens (Michx.) Sweet, Hoary Azalea-see Excluded Flora section.

Rhododendron maximum L., Great Laurel (Cohon s.n. BALT)

Rhododendron periclymenoides (Michx.) Shinners, Pink Azalea (Knapp 514 DOV)

[Azalea nudiflora L.; Rhododendron nudiflorum (L.) Torr.]

Rhododendron prinophyllum (Small) Millais, Early Azalea (Hermann 1031 UNA)

[Azalea prinophylla Small; Rhododendron roseum (Loisel.) Rehder]

Rhododendron viscosum (L.) Torr., Swamp Azalea (Knapp 2793 DOV)

[Azalea serrulata Small; A. viscosa L.; A. viscosa var. glauca (Lam.) Michx.; Rhododendron viscosum var. glaucum Torr.; R. viscosum var. serrulatum (Small) H. E. Ahles]

Vaccinium angustifolium Aiton, Late Lowbush Blueberry (Frye \& Knapp 5049A TAWES)

[Vaccinium angustifolium var. laevifolium House; V. angustifolium var. nigrum (Alph. Wood) Dole]

Vaccinium caesariense Mack., New Jersey Blueberry (Simmons \& Parrish 2623 TAWES)

Vaccinium corymbosum L., Highbush Blueberry (Knapp et al. 1809 DOV)

Vaccinium formosum S. Andrews, Southern Highbush Blueberry (Simmons \& Parrish 2593 TAWES)

Vaccinium fuscatum Aiton, Black Highbush Blueberry (Strong et al. 1859 US)

[Vaccinium atrococcum (A. Gray) Heller]

Vaccinium macrocarpon Aiton, Large Cranberry, S3/G5 (Boone s.n. TAWES)

[Oxycoccus macrocarpus (Aiton) Pursh]

Vaccinium myrtilloides Michx., Velvetleaf Blueberry, S3/G5 (Frye 5068 TAWES)

Vaccinium oxycoccos L., Small Cranberry, S2/G5 (Thompson s.n. TAWES)

[Oxycoccus ovalifolius (Michx.) A. E. Porsild]

Vaccinium pallidum Aiton, Early Lowbush Blueberry (Knapp 1191 DOV)

[Vaccinium vacillans Kalm ex Torr.]

Vaccinium stamineum L., Deerberry (Boone s.n. TAWES) [Polycodium candicans Small; Vaccinium stamineum var. interius (Ashe) E. J. Palmer \& Steyerm.; V. stamineum var. neglectum (Small) Deam]

\section{ERIOCAULACEAE}

(Pipewort Family)

Eriocaulon aquaticum (Hill) Druce, Seven-angled Pipewort, S1/G5 (Hotchkiss 7301 US)

[Eriocaulon septangulare With.]
Eriocaulon compressum Lam., Soft Pipewort, S2/G5 (Knapp 2404 TAWES)

Eriocaulon decangulare L. var. decangulare, Common Tenangled Pipewort, S1/G5 (Maxon 6469 US)

Eriocaulon parkeri B. L. Rob., Estuary Pipewort, S2/G3 (Knapp 2223 DOV)

EUPHORBIACEAE

(Spurge Family)

Acalypha gracilens A. Gray, Slender Copperleaf (Walker 4125 US)

Acalypha rhomboidea Raf., Slender Three-seeded Mercury (Hotchkiss 5537 US)

Acalypha virginica L., Virginia Copperleaf (Smith 5459 US)

- Chrozophora tinctoria (L.) A. Juss., Giradol, † Waif Reed (1964)

- Croton bonplandianus Baill., Bonpland's Croton, † Waif Reed (1964)

Croton capitatus Michx. var. capitatus, Hogwort, $†$ (Benedict s.n. US)

Croton glandulosus L. var. septentrionalis Müll. Arg., Northern Croton, † (Baltars 4124 US)

- Croton michauxii G. L. Webster, Narrowleaf Rushfoil-see Excluded Flora section.

[Crotonopsis linearis Michx.]

Croton monanthogynus Michx., Prairie Tea, † (Wherry s.n. US)

Croton willdenowii G. L. Webster, Elliptical Rushfoil (Knapp $1563 \mathrm{DOV}$ )

[Crotonopsis elliptica Willd.]

Euphorbia commutata Engelm., Wood Spurge (Reveal \& Broome 5278 MARY)

Euphorbia corollata L., Flowering Spurge (McAvoy 5927 DOV) [Euphorbia marilandica Greene]

Euphorbia cyathophora Murray, Fire-on-the-Mountain, $\dagger(\mathrm{Her}-$ mann 19352 US)

[Poinsettia cyathophora (Murray) Klotzsch \& Garcke]

Euphorbia cyparissias L., Cypress Spurge, † (Longbottom 13112 US)

Euphorbia davidii Subils, David's Spurge, † (Brown \& Gilpin s.n. NCU)

Euphorbia dentata Michx., Toothed Spurge, † (Longbottom 3726 US)

[Poinsettia dentata (Michx.) Klotzsch \& Garcke]

- Euphorbia esula L., Leafy Spurge, † Waif (Taylor s.n. NCU) [Euphorbia intercedens Podp.; E. virgata Waldst. \& Kit.]

Euphorbia falcata L., Falcate Spurge, † (Tatnall 4580 US)

Euphorbia helioscopia L., Summer Spurge, † (Knapp 27-03 DOV)

- Euphorbia herniariifolia Willd., Herniaria-leaf Spurge, † Waif Reed (1964)

- Euphorbia hirta L., Pill-pod Broomspurge, † Waif Reed (1964) [Chamaesyce hirta (L.) Millsp.; Euphorbia pilulifera L. var. discolor Engelm.] 
Euphorbia humistrata Engelm., Spreading Broomspurge (Gladu s.n. MARY)

[Chamaesyce humistrata (Engelm.) Small]

- Euphorbia hypericifolia L., Graceful Sandmat, † Waif Reed (1964)

[Chamaesyce hypericifolia (L.) Millsp.; Euphorbia glomerifera (Millsp.) L. C. Wheeler]

Euphorbia hyssopifolia L., Hyssop-leaf Broomspurge, † (Hill 8813 MARY)

[Chamaesyce hyssopifolia (L.) Small]

Euphorbia ipecacuanhae L., Wild Ipecac (Knapp 2653a DOV)

[Euphorbia arundelana Bartlett; E. marilandica Greene]

Euphorbia lathyris L., Caper Spurge, † (Baltars 225 US)

- Euphorbia lucida Waldst. \& Kit., Shining Spurge-see Excluded Flora section.

Euphorbia maculata L., Spotted Spurge (Hotchkiss 5460 US) [Chamaesyce maculata (L.) Small; C. tracyi Small; Euphorbia supina Raf.]

Euphorbia marginata Pursh, Snow-on-the-Mountain, † (Leonard 19564 US)

Euphorbia nutans Lag., Eyebane Broomspurge (Redmond 344 DOV)

[Chamaesyce nutans (Lag.) Small]

Euphorbia peplus L., Petty Spurge, † (Davis Photo Voucher MPA)

Euphorbia polygonifolia L., Seaside Spurge (Leonard 22076 US) [Chamaesyce polygonifolia (L.) Small]

- Euphorbia prostrata Aiton, Prostrate Broomspurge, † Waif Reed (1964)

[Chamaesyce prostrata (Aiton) Small; Euphorbia chamaesyce L.]

- Euphorbia pubentissima Michx., False-flowering Spurge-see Excluded Flora section.

[Euphorbia corollata L. var. zinniiflora (Small) H. E. Ahles; E. zinniiflora Small]

Euphorbia purpurea (Raf.) Fernald, Glade Spurge, S1/G3 (Tatnall 203 DOV)

Euphorbia spathulata Lam., Reticulate-seeded Spurge, S1/G5 (Boone s.n. TAWES)

[Euphorbia dictyosperma Fischer \& C. A. Mey.; E. obtusata Pursh]

- Euphorbia vermiculata Raf., Worm-seeded Spurge, † Waif Reed (1964)

[Chamaesyce vermiculata (Raf.) House]

Mercurialis annua L., Herb-mercury, † (Steele s.n. US)

- Ricinus communis L., Castor-bean, † Waif (Nibier s.n. MARY)

FABACEAE [Leguminosae]

(Pea/Bean Family)

- Aeschynomene americana L., American Joint-vetch, † Waif Reed (1964)
Aeschynomene virginica (L.) Britton, Sterns, \& Poggenb., Sensitive Joint-vetch, S1/G2, LT (McAvoy $1252 \mathrm{DOV})$

Albizia julibrissin Durazz., Silk Tree, $\ddagger$ (Longbottom 11774 US)

Amorpha fruticosa L., False Indigobush (McAvoy 5079 DOV) [Amorpha croceolanata P. W. Watson; A. curtissii Rydb.; A. virgata Small]

Amphicarpaea bracteata (L.) Fernald, American Hog Peanut (McAvoy 1568 DOV)

[Amphicarpaea bracteata var. comosa (L.) Fernald; A. pitcheri Torr. \& A. Gray]

Apios americana Medik., American Groundnut (Russell 84 US) [Apios apios (L.) MacMill.; Glycine apios L.]

- Apios priceana B. L. Rob., Traveler's Delight-see Excluded Flora section.

- Arachis bypogaea L., Peanut, † Waif Tatnall (1946)

Astragalus canadensis L. var. canadensis, Canadian Milkvetch, - S1/G5T5 (Boone s.n. TAWES)

[Astragalus carolinianus L.; A. halei Rydb.]

Astragalus distortus Torr. \& A. Gray, Ozark Milkvetch, S2/ G5 (Freeman s.n. US)

- Astragalus hamosus L., European Milkvetch, † Waif Reed (1964)

Baptisia australis (L.) R. Br., Blue Wild Indigo, S2/G5 (Ward s.n. US)

Baptisia tinctoria (L.) R. Br., Yellow Wild Indigo (Boone s.n. TAWES)

- Cajanus cajan (L.) Millsp., Congo Pea, † Waif Reed (1964)

- Cajanus scarabaeoides (L.) Thouars, Showy Pigeonpea, † Waif Reed (1964)

[Cantharospermum scarabaeoides (L.) Baill.]

- Caragana arborescens Lam., Siberian Peashrub, † Waif (Ditman s.n. MARY)—specimen most likely taken from a cultivated plant

Centrosema virginianum (L.) Benth., Coastal Butterfly Pea, - S2/G5 (Frye 6022 TAWES)

Cercis canadensis L., Eastern Redbud (Hotchkiss s.n. US)

Chamaecrista fasciculata (Michx.) Greene, Sleepingplant (Hotchkiss 5363 US)

[Cassia fasciculata Michx.; Ca. mississippiensis Pollard; Chamaecrista brachiata Pollard; C. depressa (Pollard) Greene; C. fasciculata var. macrosperma (Fernald) C. F. Reed; C. littoralis Pollard; C. mississippiensis (Pollard) Pollard ex Heller; C. robusta (Pollard) Pollard ex Heller] 
Chamaecrista nictitans (L.) Moench var. nictitans, Partridge Pea (Hill \& Klein15411 US)

[Cassia nictitans L. var. mohrii (Pollard) J. F. Macbr.; Chamaecrista aspera (Muhl. ex Elliott) Greene var. mohrii (Pollard) Pollard ex Heller; C. mohrii (Pollard) Small ex Britton \& Rose; C. multipinnata (Pollard) Greene; C. procumbens (L.) Greene]

- Cicer arietinum L., Chickpea, † Waif (s. coll., s.n. MARY)

Clitoria mariana L., Maryland Butterfly Pea (Frye 4081 TAWES) [Martusia mariana (L.) Small]

- Crotalaria pumila Ortega, Low Rattlebox, † Waif Reed (1964)

- Crotalaria rotundifolia (Walter) Poir., Rabbitbells-see Excluded Flora section. [Crotalaria angulata Mill.; C. maritima Chapm.]

Crotalaria sagittalis L., Arrowhead Rattlebox (Hotchkiss 5334 US)

Cytisus scoparius (L.) Link, Scotch Broom, † (Longbottom 11356 US)

- Dalea brachystachya A. Gray, Fort Bowie Prairie Clover, † Waif Reed (1964)

[Dalea lemmonii Parry]

- Dalea carthagenensis (Jacq.) J. F. Macbr., Cartagena Prairie Clover, † Waif Reed (1964) [Parosela floridana Rydb.]

- Desmanthus illinoensis (Michx.) MacMill., Illinois Bundleflower-see Excluded Flora section.

Desmodium canadense (L.) DC., Showy Tick-trefoil, SH/G5 (House 363 US)

Desmodium canescens (L.) DC., Hoary Tick-trefoil (Killip 13315 US)

Desmodium ciliare (Muhl. ex Willd.) DC., Hairy Small-leaved Tick-trefoil (Frye 732 TAWES)

Desmodium cuspidatum (Muhl. ex Willd.) DC. ex G. Don var. cuspidatum, Toothed Tick-trefoil, S1/G5T5? (Frye 654 TAWES)

Desmodium fernaldii B. G. Schub., Fernald's Tick-trefoil, S1/ G4 (McAvoy 1763 DOV)

Desmodium glabellum (Michx.) DC., Dillenius' Tick-trefoil (Hotchkiss 5578 US)

Desmodium humifusum (Muhl. ex Willd.) L. C. Beck, Trailing Tick-trefoil, SH/G1G2Q (Canby s.n. PH)

Desmodium laevigatum (Nutt.) DC., Smooth Tick-trefoil, S3/ G5 (Frye 650 TAWES)

Desmodium lineatum DC., Sand Tick-trefoil, SH/G5 (Canby s.n. DOV)

Desmodium marilandicum (L.) DC., Maryland Tick-trefoil (Uhler s.n. US)
Desmodium nuttallii (Schindl.) B. G. Schub., Nuttall's Ticktrefoil, S1?/G5 (Frye 714 TAWES)

Desmodium obtusum (Muhl. ex Willd.) DC., Stiff Tick-trefoil, S1/G4G5 (Cooley s.n. TAWES)

[Desmodium rigidum (Elliott) DC.]

Desmodium ochroleucum M. A. Curtis, Cream Tick-trefoil, - S1/G2 (Boone s.n. TAWES)

Desmodium paniculatum (L.) DC. var. paniculatum, Panicledleaved Tick-trefoil (Cooley s.n. TAWES)

Desmodium perplexum B. G. Schub., Perplexed Tick-trefoil (Lea 852 TAWES)

Desmodium rotundifolium DC., Prostrate Tick-trefoil (Steury 040710.1 US)

Desmodium sessilifolium (Torr.) Torr. \& A. Gray, Sessile-Leaved Tick-trefoil, SH/G5 (Abbott 2478 US)

Desmodium strictum (Pursh) DC., Pineland Tick-trefoil, S1/G4 (McAvoy 1762 DOV)

- Desmodium tenuifolium Torr. \& A. Gray, Slim-leaved Tick-trefoil-see Excluded Flora section.

Desmodium viridiflorum (L.) DC., Velvety Tick-trefoil (Steury 050820.1 US)

Galactia brachypoda Torr. \& A. Gray, Lax Milkpea (Plitt 76 $\mathrm{MO})$

Galactia regularis (L.) Britton, Sterns, \& Poggenb., Eastern Milkpea (Lea 845 TAWES)

- Galactia volubilis (L.) Britton var. volubilis, Downy Milkpea-see Excluded Flora section.

[Galactia macreei M. A. Curtis]

- Galega officinalis L., Common Milkpea, † Waif Reed (1964)

- Genista tinctoria L., Dryer's Greenweed-see Excluded Flora section.

Gleditsia triacanthos L., Honey-locust (Killip s.n. US)

Glycine max (L.) Merr., Soybean, † (Boone s.n. TAWES)

Gymnocladus dioica (L.) K. Koch, Kentucky Coffeetree (Cooley s.n. TAWES)

Hylodesmum glutinosum (Muhl. ex Willd.) H. Ohashi \& R. R. Mill, Large Tick-trefoil (Frye 655 TAWES) [Desmodium glutinosum (Muhl. ex Willd.) Alph. Wood]

Hylodesmum nudiflorum (L.) H. Ohashi \& R. R. Mill, Nakedflowered Tick-trefoil (Frye 4085 TAWES)

[Desmodium nudiflorum (L.) DC.]

Hylodesmum pauciflorum (Nutt.) H. Ohashi \& R. R. Mill, Fewflowered Tick-trefoil, S2/G5 (Steury 021009.5 US) [Desmodium pauciflorum (Nutt.) DC.]

- Indigofera trifoliata L., Three-leaf Indigo, † Waif Reed (1964) 
Kummerowia stipulacea (Maxim.) Makino, Korean Clover, † (Longbottom 6629 DOV)

[Lespedeza stipulacea Maxim.]

Kummerowia striata (Thunb.) Schindl., Common Korean Clover, † (Tatnall 2431 DOV)

[Lespedeza striata (Thunb.) Hook. \& Arn.]

Lablab purpureus (L.) Sweet, Hyacinth-bean-see Excluded Flora section.

[Dolichos lablab L.]

- Lathyrus aphaca L., Yellow Peavine, † Waif Reed (1964)

Lathyrus hirsutus L., Caley Pea, † (Longbottom 23044 MARY)

- Lathyrus inconspicuus L., Inconspicuous Pea, † Waif Reed (1964)

Lathyrus latifolius L., Perennial Pea, † (Leonard 22071 US)

Lathyrus palustris L., Marsh Pea, S1/G5 (Knapp 2604 TAWES)

- Lathyrus sativus L., White Pea, † Waif Reed (1964)

Lathyrus venosus Muhl. ex Willd., Smooth Veiny Peavine (Boone s.n. TAWES)

- Lens culinaris Medik., Lentil, † Waif Reed (1964)

Lespedeza angustifolia (Pursh) Elliott, Narrow-leaf Bushclover (Longbottom 10389 DOV)

Lespedeza bicolor Turcz., Shrubby Bushclover, $\ddagger$ (Steury 031004.3 US)

Lespedeza capitata Michx., Round-headed Bushclover (O’Neil s.n. DOV)

Lespedeza cuneata (Dum. Cours.) G. Don, Chinese Bushclover, $\ddagger$ (Windler et al. 4134 DOV)

Lespedeza frutescens (L.) Hornem., Shrubby Bushclover, S3/ G5 (Redmond 400 DOV)

[Lespedeza violacea auct. non (L.) Pers.]

Lespedeza hirta (L.) Hornem. ssp. hirta, Hairy Bushclover (McAvoy 6713 DOV)

Lespedeza procumbens Michx., Trailing Bushclover (Redmond 399 DOV)

Lespedeza repens (L.) W. P. C. Barton, Creeping Bushclover (Herliby s.n. DOV)

Lespedeza stuevei Nutt., Tall Bushclover, S3/G5 (Longbottom $6600 \mathrm{DOV})$

Lespedeza thunbergii (DC.) Nakai, Thunberg's Bushclover, † (Naczi 8956 DOV)

Lespedeza violacea (L.) Pers., Violet Bushclover (McAvoy 5321 DOV)

[Lespedeza intermedia (S. Watson) Britton]

Lespedeza virginica (L.) Britton, Slender Bushclover (Tatnall 4663 DOV)

Lotus corniculatus L., Garden Bird's-foot Trefoil, † (Longbottom 11568 US)
Lotus tenuis Waldst. \& Kit. ex Willd., Slender Trefoil, † (Longbottom $15582 \mathrm{NY}$ )

[Lotus glaber Mill.]

Lupinus perennis L. ssp. perennis, Sundial Lupine, S2/G5T5 (Lea 2340 TAWES)

[Lupinus gracilis Nutt.; L. nuttallii S. Watson; L. perennis ssp. gracilis (Nutt.) Chapm.]

- Lupinus polyphyllus Lindl., Bigleaf Lupine, † Waif (Eggleston 21401 US)

- Medicago heldreichii E. Small, Heldreich's Alfalfa, † Waif Reed (1964)

[Trigonella polycarpa Boiss. \& Heldr.]

Medicago lupulina L., Black Medic, † (Fosberg 23372 US)

- Medicago minima (L.) L., Burr Medick, † Waif Reed (1964)

- Medicago monspeliaca (L.) Trautv., Hairy Medic, † Waif Reed (1964)

[Trigonella monspeliaca L.]

- Medicago orbicularis (L.) Bartal., Button Medic, † Waif Reed (1964)

Medicago sativa L., Alfalfa, † (Longbottom 11854 US)

- Medicago scutellata (L.) Mill., Snail Medick, † Waif Reed (1964)

Melilotus albus Medik., White Sweetclover, † (Longbottom 9401 DOV)

Melilotus officinalis (L.) Pall., Yellow Sweetclover, † (Longbottom 5173 DOV)

- Mimosa pudica L., Sensitive Plant, † Waif Reed (1964)

- Ononis campestris W. D. J. Koch \& Ziz., Spiny Restharrow, † Waif Reed (1964)

[Ononis spinosa L.]

- Ononis repens L., Common Restharrow, † Waif (Plitt s.n. US)

Orbexilum psoralioides (Walter) Vincent, False Scurfpea, SX/ G5T4 (Sherard s.n. OXF) [Psoralea psoraloides (Walter) Cory]

Brown et al. (1987) reported this taxon as O. pedunculatum (Walter) Vincent, but the specimen was annotated by Reveal, a coauthor on the Brown et al. (1987) paper, as Psoralea psoraloides (Walter) Cory in 1982. We agree with Reveal's annotation.

Phaseolus polystachios (L.) Britton, Sterns, \& Poggenb., Wild Kidney Bean, S3/G5 (McAvoy 6715 DOV)

Phaseolus vulgaris L., Kidney Bean, † (Turpin 459 MARY)

- Pisum fulvum Sm., Tawny Pea, † Waif Reed (1964)

- Pisum sativum L., Garden Pea, † Waif (s. coll., s.n. MARY) 
Pueraria montana (Lour.) Merr. var. lobata (Willd.) Maesen \& S. M. Almeida ex Sanjappa \& Predeep, Kudzu, $\ddagger$ (Longbottom 7848 DOV)

[Pueraria lobata (Willd.) Ohwi; P. thunbergiana Benth.]

Rhynchosia tomentosa (L.) Hook. \& Arn. var. tomentosa, Hairy Snoutbean, S2/G5 (Knapp 03-01 TAWES)

Robinia hispida L., Bristly Locust, †? (Tatnall 3313 DOV) [Robinia grandiflora L.; R. hispida var. rosea Pursh; R. pallida Ashe; R. speciosa Ashe]

Robinia pseudoacacia L., Black Locust (Boone s.n. TAWES)

Robinia viscosa Vent., Clammy Locust (Longbottom 11357 US)

- Securigera cretica (L.) Lassen, Cretan Crown-vetch, † Waif Reed (1964)

[Coronilla cretica L.]

Securigera varia (L.) Lassen, Common Crown-vetch, † (Hermann 11836 US)

[Coronilla varia L.]

- Senna baubinioides (A. Gray) H. S. Irwin \& Barneby, Shrubby Wild Sensitive-plant, † Waif Reed (1964)

[Cassia bauhinioides A. Gray]

Senna hebecarpa (Fernald) H. S. Irwin \& Barneby, Wild Senna (Steury 980724.7 US)

[Cassia hebecarpa Fernald]

- Senna italica Mill., Port Royal Wild Sensitive-plant, † Waif Reed (1964)

[Cassia obovata Collad.]

Senna marilandica (L.) Link, Maryland Senna, S3/G5 (Redmond 428 DOV)

[Cassia marilandica L.]

Senna obtusifolia (L.) H. S. Irwin \& Barneby, Blunt-leaf Senna, $\ddagger$ (Longbottom 10326 DOV)

[Cassia obtusifolia L.; C. torta L.]

- Senna occidentalis (L.) Link, Septicweed-see Excluded Flora section.

[Cassia occidentalis L.]

- Sesbania herbacea (Mill.) McVaugh, Big-pod Sesbania, † Waif Reed (1964)

[Sesban exaltatus (Raf.) Rydb.; Sesbania exaltata (Raf.) Rydb. ex A. W. Hill; S. macrocarpa Muhl.]

Strophostyles helvola (L.) Elliott, Trailing Wild Bean (Frye 709 TAWES)

[Phaseolus helvolus L.]

Strophostyles leiosperma (Torr. \& A. Gray) Piper, Slick-seed Wild Bean, † (Steury 98083.9 US)

[Strophostyles pauciflora S. Watson]

Strophostyles umbellata (Muhl. ex Willd.) Britton, Pink Wild Bean (Frye \& Harrison s.n. TAWES)

[Phaseolus umbellatus (Muhl. ex Willd.) Britton; Strophostyles umbellata var. paludigena Fernald]
Stylosanthes biflora (L.) Britton, Sterns, \& Poggenb., Pencilflower (Boone s.n. TAWES) [Stylosanthes biflora var. hispidissima (Michx.) Pollard \& Ball; S. riparia Kearney]

- Styphnolobium japonicum (L.) Schott, Japanese Pagoda Tree, † Waif ([collector illegible] s.n. MARY)

[Sophora japonica L.]

- Tephrosia purpurea (L.) Pers., Fishpoison, † Waif Reed (1964)

Tephrosia spicata (Walter) Torr. \& A. Gray, Spiked Hoary-pea, - S1S2/G4G5 (Knapp \& Maloof 16-01 TAWES)

Tephrosia virginiana (L.) Pers., Goat's-rue (Frye 4077 TAWES)

Thermopsis villosa (Walter) Fernald \& B. G. Schub., Hairy False Lupine, † (Hermann 11837 US)

[Thermopsis caroliniana M. A. Curtis]

Trifolium arvense L., Rabbitfoot Clover, † (Hotchkiss 5224 US)

Trifolium aureum Pollich, Yellow Clover, † (Hotchkiss 5218 US) [Trifolium agrarium L.]

Trifolium campestre Schreb., Low Hop Clover, † (Hotchkiss 5157 US)

[Trifolium procumbens L.]

Trifolium dubium Sibth., Suckling Clover, † (Tidestrom 5834 DOV)

- Trifolium fragiferum L., Strawberry Clover, † Waif (McBryde s.n. MARY)

Trifolium hybridum L., Alsike Clover, † (Fosberg 23383 US)

Trifolium incarnatum L., Crimson Clover, † (Longbottom 8651 DOV)

[Trifolium incarnatum var. elatius Gibelli \& Belli]

Trifolium medium L., Zigzag Clover, † (McBryde s.n. MARY)

Trifolium pratense L., Red Clover, † (Longbottom 11549 DOV)

Trifolium reflexum L., Buffalo Clover, SH/G3G4 (Pollard s.n. US)

[Trifolium reflexum var. glabrum Lojac.]

Trifolium repens L., White Clover, † (Hotchkiss 5832 US)

Trifolium resupinatum L., Reversed Clover, † (Norton s.n. MARY)

Trifolium virginicum Small ex Small \& Vail, Kate's Mountain Clover, S2S3/G3 (Reveal \& Broome 5223 MARY)

- Trigonella caerulea (L.) Ser., Blue-white Trigonella, † Waif Reed (1964)

- Trigonella foenum-graecum L., Sicklefruit Fenugreek Trigonella, $†$ Waif Reed (1964)

- Vachellia constricta (Benth.) Seigler \& Ebinger, Whitethorn Acacia, † Waif Reed (1964)

[Acacia constricta Benth.]

- Vicia americana Muhl. ex Willd. var. americana, American Purple Vetch, SH/G5T5-see Excluded Flora section. 
Vicia caroliniana Walter, Carolina Wood Vetch (Frye 1000 TAWES)

[Vicia hugeri Small]

Vicia cracca L., Tufted Vetch, † (Longbottom 8768 DOV)

- Vicia faba L., Fava Bean, † Waif rarely escaping from cultivation (Steele s.n. US)

Vicia grandiflora Scop., Showy Vetch, † (Hermann 23589 US)

Vicia hirsuta (L.) Gray, Tiny Vetch, † (Longbottom 8524 DOV)

Vicia lathyroides L., Spring Vetch (Longbottom 10858 DOV)

Vicia narbonensis L., Narbonne Vetch, † (Maxon \& Standley 299 US)

- Vicia peregrina L., Wandering Vetch, † Waif Reed (1964)

Vicia sativa L. ssp. sativa, Spring Vetch, $\ddagger$ (Hotchkiss 5879 US) [Vicia sativa ssp. nigra (L.) Ehrh.; V. angustifolia L.]

Vicia tetrasperma (L.) Moench, Lentil Vetch, † (Sanborn s.n. MARY)

Vicia villosa Roth ssp. villosa, Shaggy Vetch, † (O’Neil s.n. MARY)

Vicia villosa Roth ssp. varia (Host) Corb., Winter Vetch, † (Dingee s.n. MARY)

[Vicia dasycarpa Ten.]

- Vigna mungo (L.) Hepper, Black Gram, † Waif Reed (1964) [Phaseolus mungo L.]

Vigna unguiculata (L.) Walp., Black-eyed Pea, † (Norton s.n. MARY)

[Vigna catjang (L.) Walp.; V. sinensis (L.) Savi ex Hassk.]

Wisteria floribunda (Willd.) DC., Japanese Wisteria, † (Steury 021003.4 US)

Wisteria frutescens (L.) Poir., American Wisteria, † (Strong et al. 1870 US)

Wisteria sinensis (Sims) Sweet, Chinese Wisteria, $\ddagger$ (Leonard 21106 US)

\section{FAGACEAE}

(Beech Family)

Castanea dentata (Marshall) Borkh., American Chestnut, S2S3/G4 (Knapp 112-03 TAWES)

Castanea pumila (L.) Mill., Allegheny Chinquapin (Wiegand s.n. TAWES)

[Castanea ashei (Sudw.) Sudw.]

Fagus grandifolia Ehrh., American Beech (Uhler s.n. US)

- Fagus sylvatica L., European Beech-see Excluded Flora section.

Quercus acutissima Curruth., Sawtooth Oak, † (Longbottom 12279 DOV)

Quercus alba L., White Oak (Kolb 968 MARY)

Quercus bicolor Willd., Swamp White Oak (Frye \& Knapp 5417 TAWES)

Quercus coccinea Münchh., Scarlet Oak (Frye 719 TAWES)

[Quercus coccinea var. tuberculata Sarg.]
Quercus falcata Michx., Southern Red Oak (Frye 720 TAWES)

[Quercus digitata Sudw.]

Quercus ilicifolia Wagenh., Scrub Oak (Bartlett 3066 US)

Quercus imbricaria Michx., Shingle Oak (Wiegand s.n. TAWES)

- Quercus laurifolia Michx., Laurel Oak-see Excluded Flora section.

Quercus lyrata Walter, Overcup Oak (Frye s.n. TAWES)

Quercus macrocarpa Michx., Bur Oak, S1S2/G5 (Lea 898 TAWES)

Quercus marilandica Münchh., Blackjack Oak (Frye 5562 TAWES)

Quercus michauxii Nutt., Swamp Chestnut Oak (Walker 5561 US)

Quercus montana Willd., Chestnut Oak (Frye 722 TAWES) [Quercus prinus L.]

Quercus mublenbergii Engelm., Chinquapin Oak (Naczi 9324 DOV)

[Quercus acuminata (Michx.) Houba \& Sarg.; Q. prinoides Willd. var. acuminata (Michx.) Gleason]

Quercus nigra L., Water Oak (Cooley s.n. TAWES)

Quercus pagoda Raf., Cherrybark Oak (Davis 5034c TAWES) [Quercus falcata Michx. var. pagodifolia Elliott; Q. pagodifolia (Elliott) Ashe]

Quercus palustris Münchh., Pin Oak (Ubler s.n. US)

Quercus phellos L., Willow Oak (Baltars 1214 US)

Quercus prinoides Willd., Dwarf Chinquapin Oak (Knapp 2716 DOV)

Quercus rubra L., Northern Red Oak (Engh s.n. MARY) [Quercus maxima (Marshall) Ashe; Q. rubra var. borealis (Michx.) Farw.]

Quercus shumardii Buckley, Shumard Oak, S2/G5 (Wiegand s.n. TAWES) [Quercus schneckii Britton]

Quercus stellata Wangenh., Post Oak (Hotchkiss 5642 US) [Quercus minor (Marshall) Sarg.]

Quercus velutina Lam., Black Oak (Engh s.n. MARY)

GENTIANACEAE

(Gentian Family)

Bartonia paniculata (Michx.) Muhl. ssp. paniculata, Twining Screwstem, S3/G5T5 (Cooley 921020-1 TAWES) [Bartonia lanceolata Small]

Bartonia virginica (L.) Britton, Sterns, \& Poggenb., Yellow Screwstem (Boone s.n. TAWES)

Centaurium erythraea Raf., Common Centaury, † (Frye s.n. TAWES) [Centaurium umbellatum Raf.]

Centaurium pulchellum (Sw.) Druce, Branching Centaury, $\dagger$ (Cohen 3 US)

- Gentiana alba Muhl. ex Nutt., Yellow Gentian-see Excluded Flora section.

Gentiana andrewsii Griseb. var. andrewsii, Fringe-top Bottle Gentian, S2/G5? (Steury 021009.2 US) 
- Gentiana autumnalis L., Pine Barren Gentian-see Excluded Flora section.

Gentiana catesbyi Walter, Elliott's Gentian (Naczi 8946 DOV) [Dasystephana latifolia (Chapm.) Small; D. parvifolia (Chapm.) Small; Gentiana elliottii Chapm.]

Gentiana clausa Raf., Closed Gentian (Toppin s.n. US)

Gentiana linearis Froel., Narrowleaf Gentian, S3/G4G5 (Boone s.n. TAWES)

Gentiana puberulenta J. S. Pringle, Downy Gentian, SH/ G4G5 (Steele $212 \mathrm{~F}$ )

Gentiana saponaria L., Soapwort Gentian (Davis 2622 TAWES) [Dasystephana puberula (Michx.) Small; D. saponaria (L.) Small; Gentiana puberula Michx.]

Gentiana villosa L., Striped Gentian, S1/G4 (Steury 981007.6 US)

[Dasystephana villosa (L.) Small]

Gentianella quinquefolia (L.) Small ssp. quinquefolia, Stiff Gentian, S1/G5 (Boone s.n. TAWES)

Gentianopsis crinita (Froel.) Ma, Fringed Gentian, S1/G5 (Fessenden 5464 US)

[Gentiana crinita Froel.; G. ventricosa Griseb.; Gentianella crinita (Froel.) G. Don]

Obolaria virginica L., Virginia Pennywort (Knapp 2371 DOV)

Sabatia angularis (L.) Pursh, Square-stemmed Rose Pink (Boone s.n. TAWES)

Sabatia campanulata (L.) Torr., Slender Marsh Pink, S1/G5 (Knapp 42-02 TAWES)

Sabatia difformis (L.) Druce, Lanceleaf Rose Gentian, S1/ G4G5 (Knapp 32-02 TAWES)

Sabatia dodecandra (L.) Britton, Sterns, \& Poggenb., Large Marsh Pink, S3/G5? (Cooley s.n. TAWES)

Sabatia stellaris Pursh, Sea Pink (Shetler s.n. US)

Schenkia spicata (L.) G. Mans., Spiked Centaury, † (Tatnall $4589 \mathrm{PH})$

[Centaurium spicatum (L.) Fritsch]

\section{GERANIACEAE}

(Geranium Family)

Erodium cicutarium (L.) L'Hér., Pin Clover, † (Higgins s.n. MARY)

Geranium carolinianum L., Carolina Cranesbill (Longbottom \& Hollway 11362 US)

Geranium columbinum L., Long-stalked Cranesbill, † (Smith 5276 US)

Geranium dissectum L., Cutleaf Cranesbill, † (Cusick s.n. CM)

Geranium maculatum L., Wild Cranesbill (Ubler s.n. US)

Geranium molle L., Dovefoot Cranesbill, † (Frost 9 MARY)
- Geranium pratense L., Meadow Geranium-see Excluded Flora section.

Geranium pusillum L., Small-flowered Cranesbill, † (Walker 2437 MARY)

Geranium robertianum L., Herb Robert, S1/G5 (Knapp 5130 TAWES)

- Geranium rotundifolium L., Roundleaf Geranium, † Waif Reed (1964)

- Geranium sibiricum L., Siberian Geranium, † Waif (Fessenden 5416 US)

Geranium thunbergii Siebold \& Zucc. ex Lindl. \& Paxton, Dewdrop Cranesbill, $\uparrow$ (Hill 10818 MARY)

[Geranium nepalense Sweet var. thunbergii (Seibold \& Zucc. ex Lind. \& Paxton) Kudo]

GROSSULARIACEAE

(Gooseberry Family)

Ribes americanum Mill., Wild Black Currant, S1/G5 (Norton s.n. MARY)

Ribes aureum Pursh, Golden Currant, † (Norton s.n. US)

Ribes cynosbati L., Prickly Gooseberry, S3/G5 (Hill 10275 MARY)

[Grossularia cynosbati (L.) Mill.]

Ribes glandulosum Grauer, Skunk Currant, S3/G5 (Boone s.n. TAWES)

- Ribes birtellum Michx., Smooth Gooseberry-see Excluded Flora section.

Ribes missouriense Nutt., Missouri Gooseberry, †? (Miller 44 MARY)

- Ribes nigrum Michx., European Black Currant-see Excluded Flora section.

Ribes rotundifolium Michx., Appalachian Gooseberry (Jennings s.n. CM)

[Grossularia rotundifolia (Michx.) Coville \& Britton]

Ribes rubrum L., Northern Red Currant, † (Norton s.n. MARY) [Ribes sativum Syme]

Ribes uva-crispa L., European Gooseberry, † (Steury 070919.1 US)

HAEMODORACEAE

(Bloodwort Family)

Lachnanthes caroliniana (Lam.) Dandy, Red Root, S1/G4 (Knapp \& Smith 26-02 TAWES)

\section{HALORAGACEAE}

(Watermilfoil Family)

Myriophyllum aquaticum (Vell.) Verdc., Parrot Feather Watermilfoil, $\ddagger$ (Killip 36995 US)

[Myriophyllum brasiliense Cambess.; M. proserpinacoides Gillies ex Hook. \& Arn.]

Myriophyllum beterophyllum Michx., Broadleaf Watermilfoil, S1/G5 (Uhler s.n. US)

Myriophyllum bumile (Raf.) Morong, Low Watermilfoil (Naczi \& Davis 598 DOV)

Myriophyllum pinnatum (Walter) Britton, Sterns, \& Poggenb., Cutleaf Watermilfoil (Hotchkiss 8016 US) 
Myriophyllum sibiricum Kom., Shortspike Watermilfoil (Shull 327 US)

[Myriophyllum exalbescens Fernald]

Myriophyllum spicatum L., Eurasian Watermilfoil, $\ddagger$ (Coville 110 US)

Myriophyllum tenellum Bigelow, Slender Watermilfoil, SH/ G5 (Stewart s.n. MARY)

Myriophyllum verticillatum L., Whorl-Leaf Watermilfoil, S1/ G5 (McCarthy s.n. TAWES)

Proserpinaca palustris L., Marsh Mermaidweed (Knapp 1067 DOV)

[Proserpinaca intermedia Mack; P. palustris var. crebra Fernald \& Griscom]

Proserpinaca pectinata Lam., Combleaf Mermaidweed (Pennell 2526 US)

\section{HAMAMELIDACEAE}

(Witch-hazel Family)

Hamamelis virginiana L., American Witch-hazel (Longbottom et al. 11793 US)

\section{HYDRANGEACEAE}

(Hydrangea Family)

Deutzia gracilis Siebert \& Zucc., Slender Pride of Rochester, $\dagger$ (Miller s.n. US)

Deutzia scabra Thunb., Fuzzy Deutzia, † (Dorsey 008 MARY)

Hydrangea arborescens L., Wild Hydrangea (Knapp 2840 DOV) [Hydrangea arborescens var. cordata (Pursh) Torr. \& A. Gray]

Philadelphus coronarius L., Sweet Mock Orange, † (Daniel s.n. MARY)

Philadelphus hirsutus Nutt., Streambank Mock Orange, † (Windler \& Lombaro 3778 DOV)

Philadelphus inodorus L., Scentless Mock Orange, † (Kelly 32 MARY)

[Philadelphus gloriosus Beadle; P. grandiflorus Willd.]

Philadelphus pubescens Loisel., Hoary Mock Orange, $†$ (Hayden 231A NCU)_possibly merely persisting after planting [Philadelphus intectus Beadle]

\section{HYDROCHARITACEAE}

(Tape Grass Family)

Egeria densa Planch., Brazilian Waterweed, $\ddagger$ (Baltars 788 US) [Elodea densa (Planch.) Casp.; Philotria densa (Planch.) Small]

Elodea canadensis Michx., Common Waterweed (Hotchkiss s.n. US)

[Philotria canadensis (Michx.) Britton]

Elodea nuttallii (Planch). H. St. John, Nuttall's Waterweed (Killip 32656 US)
Hydrilla verticillata (L. f.) Royle, Hydrilla, $\ddagger$ (Steury 090925.5 US)

Limnobium spongia (Bosc) Rich. ex Steud., American Frog's-bit, - S1/G4 (Steury 020723.1 US)

Najas canadensis Michx., Canadian Waternymph (McAtee 2289 US)

Najas flexilis (Willd.) Rostk. \& W. L. E. Schmidt, Nodding Waternymph (Leonard \& Killip 602 US)

Najas gracillima (Braun ex Engelm.) Magnus, Slender Waternymph (McAtee 2766 US)

Najas guadalupensis (Spreng.) Magnus ssp. guadalupensis, Southern Waternymph (Steury 980722.2 US)

Najas minor All., Brittle Waternymph, † (Redman 6789 BALT)

Vallisneria americana Michx., Vallisneria (Killip 31975 US) [Vallisneria spiralis L.]

HYDROLEACEAE

(Waterpod Family)

Hydrolea quadrivalvis Walter, Waterpod, †? (Frye 970819FR DOV)

[Nama quadrivalvis (Walter) Kuntze]

HYPERICACEAE

(St. John's-wort Family)

Hypericum adpressum W. P. C. Barton, Creeping St. John'swort, S1/G3 (McAvoy 5696 DOV)

Hypericum androsaemum L., Sweet Amber, † (Knapp 3069 MARY)

- Hypericum ascyron L. ssp. pyramidatum (Aiton) N. Robson, Great St. John's-wort—see Excluded Flora section.

Hypericum boreale (Britton) E. P. Bicknell, Northern St. John'swort (Ebert \& Holt 220EH DOV)

Hypericum canadense L., Canadian St. John's-wort (Naczi 11554 DOV)

Hypericum crux-andreae (L.) Crantz, St. Peter's-wort (Dowell 6464 US)

[Ascyrum stans Michx. ex Willd.; Hypericum stans W. P. Adams \& N. Robson]

Hypericum densiflorum Pursh, Bushy St. John's-wort (Hotchkiss \& Ubler 7331 US)

[Hypericum glomeratum Small]

Hypericum denticulatum Walter var. denticulatum, Coppery St. John's-wort, S2/G5TNR (Knapp \& Longbottom 1594 DOV)

Hypericum drummondii (Grev. \& Hook.) Torr. \& A. Gray, Drummond's St. John's-wort, SH/G5 (Earle 4012 PH) [Sarothra drummondii Grev. \& Hook.]

Hypericum ellipticum Hook., Pale St. John's-wort, SU/G5 (Pennell $1571 \mathrm{PH}$ )

Hypericum fraseri Spach, Fraser's Marsh St. John's-wort (Knapp \& Hall 5209 TAWES)

[Triadenum fraseri (Spach) Gleason] 
Hypericum gentianoides (L.) Britton, Sterns, \& Poggenb., Orange-grass St. John's-wort (Naczi 11558 DOV) [Sarothra gentianoides L.]

Hypericum gymnanthum Engelm. \& A. Gray, Claspingleaf St. John's-wort, S3/G4 (Knapp 581 DOV)

Hypericum bypericoides (L.) Crantz, St. Andrew's-cross (Dill 234 DOV)

[Acyrum hypericoides L.; A. bypericoides var. multicaule (Michx. ex Willd.) Fernald; A. linifolium Spach; A. multicaule Michx.; Hypericum stragulum W. P. Adams \& N. Robson]

- Hypericum mitchellianum Rydb., Blue Ridge St. John'swort-see Excluded Flora section.

Hypericum mutilum L., Slender St. John's-wort (Roller 692 US)

Hypericum perforatum L., Common St. John's-wort, † (McCarthy s.n. US)

Hypericum prolificum L., Shrubby St. John's-wort (Baltars 631 US)

Hypericum punctatum Lam., Common St. John's-wort (Randall $J r .45$ US)

[Hypericum subpetiolatum E. P. Bicknell ex Small]

Hypericum stragulum W. P. Adams \& N. Robson, Low St. John's-wort (McAvoy 5235 DOV)

- Hypericum tubulosum Walter, Large Marsh St. John'swort-see Excluded Flora section.

[Triadenum tubulosum (Walter) Gleason]

Hypericum virgatum Lam., Sharpleaf St. John's-wort, SH/ G4? (Wherry s.n. US)

[Hypericum acutifolium Elliott; H. denticulatum Walter var. acutifolium (Elliott) S. F. Blake; H. virgatum Lam. var. acutifolium (Elliott) J. M. Coult.]

Hypericum virginicum L., Marsh St. John's-wort (Strong \& Simmons 3203 US)

[Triadenum virginicum (L.) Raf.]

Hypericum walteri J. F. Gmel., Walter's St. John's-wort (Hotchkiss 5916 US)

[Triadenum walteri (J. F. Gmel.) Gleason]

\section{HYPOXIDACEAE}

(Stargrass Family)

Hypoxis hirsuta (L.) Coville, Common Stargrass (Knowlton \& Britt s.n. US)

IRIDACEAE

(Iris Family)

Crocus vernus (L.) Hill, Dutch Crocus, † (Hill 9738 MARY)

Iris cristata Aiton, Dwarf Crested Iris, S1/G5 (Steele s.n. US) [Lophiris cristata (Aiton) M. B. Crespo, Mart.-Azorín \& Mavrodiev]

Iris domestica (L.) Goldblatt \& Mabb., Blackberry Lily, † (Leonard 1261 US)

[Belamcanda chinensis (L.) Redouté.; Gemmingia chinensis

(L.) Kuntze]
- Iris ensata Thunb., Japanese Iris-see Excluded Flora section. [Iris kaempferi Siebold ex Lem.; Limniris ensata (Thunb.) Rodion.]

Iris germanica L., German Iris, † (Benzinger 13 MARY)

Iris prismatica Pursh, Slender Blue Iris, S1/G4G5 (Knapp 2589 TAWES)

[Limniris prismatica (Pursh) Rodion.]

Iris pseudacorus L., Yellow Flag, † (Flemming 2470 US) [Limniris pseudacorus (L.) Fuss]

Iris verna L., Dwarf Iris, S1/G5 (Ditman s.n. MARY)

[Gattenhofia verna (L.) Medik.; Iris verna var. smalliana Fernald ex M. E. Edwards]

Iris versicolor L., Northern Blue Iris (Beyersdorfer 42 US) [Limniris versicolor (L.) Rodion.]

Iris virginica L., Southern Blue Flag, S3/G5 (Pollard 302 US) [Iris shrevei Small; I. virginica var. shrevei (Small) E. S. Anderson; Limniris virginica (L.) Rodion.]

Sisyrinchium angustifolium Mill., Narrow-leaved Blue-eyed Grass (Allard s.n. US)

[Sisyrinchium graminoides E. P. Bicknell]

Sisyrinchium arenicola E. P. Bicknell, Sandyland Blue-eyed Grass, S1/G5? (Frye 5530 DOV)

Sisyrinchium atlanticum E. P. Bicknell, Atlantic Blue-eyed Grass (Hotchkiss 5217 US)

[Sisyrinchium mucronatum Michx. var. atlanticum (E. P. Bicknell) H. E. Ahles]

Sisyrinchium montanum Greene, Strict Blue-eyed grass (Eirfrig s.n. CM)

Sisyrinchium mucronatum Michx., Needle-tip Blue-eyed Grass (Baltars 589 US)

ITEACEAE

(Sweetspire Family)

Itea virginica L., Virginia Willow (Norton s.n. MARY)

JUGLANDACEAE

(Walnut Family)

Carya cordiformis (Wangenh.) K. Koch, Bitternut Hickory (Naczi 9326 DOV)

Carya glabra (Mill.) Sweet, Sweet Pignut Hickory (Norton s.n. MARY)

Carya illinoinensis (Wangenh.) K. Koch, Pecan, † (Longbottom $14166 \mathrm{NY})$

Carya laciniosa (F. Michx.) Loudon, Big Shellbark Hickory, S1/G5 (Lea 860 TAWES)

Carya ovalis (Wangenh.) Sarg., Red Hickory (McAvoy 5479 DOV)

Carya ovata (Mill.) K. Koch, Shagbark Hickory (Riefner 81-96 MARY)

Carya pallida (Ashe) Engl. \& Grab., Sand Hickory (Steury 990904.5 US) 
Carya tomentosa (Lam.) Nutt., Mockernut Hickory (Tidestrom \& Bartlett 4660 US)

[Carya alba (L.) Nutt.]

Juglans ailantifolia Carrière, Japanese Walnut, † (Wixted Photo Voucher MPA)

Juglans cinerea L., Butternut, S2S3/G4 (Steury 980821.4 US) Juglans nigra L., Black Walnut (Hitchcock 12659 US)

\section{JUNCACEAE}

(Rush Family)

Juncus acuminatus Michx., Sharp-fruited Rush (Knapp 2638 DOV)

Juncus anthelatus (Weigand) R. E. Brooks, Large Path Rush (Fosberg 55580 US)

[Juncus tenuis Willd. var. anthelatus Wiegand]

Juncus articulatus L., Jointed Rush, S1/G5 (Knapp 2638 Assateague Island National Seashore Herbarium)

Juncus balticus Willd. var. littoralis Engelm., Baltic Rush, SH/ G5T5 (Baltars 4497 Cylburn Arboretum)

[Juncus arcticus ssp. balticus (Willd.) Hyl.; J. arcticus Willd. var. littoralis (Engelm.) B. Boivin]

Juncus biflorus Elliott, Large Grass-leaved Rush (Knapp 600 DOV)

[Juncus marginatus Rostk. var. biflorus (Elliott) Chapm.]

Juncus brachycarpus Engelm., Short-fruited Rush (Knapp 1614 DOV)

- Juncus brachycephalus (Engelm. ex A. Gray) Buchenau, Smallheaded Rush-see Excluded Flora section.

Juncus brevicaudatus (Engelm.) Fernald, Narrow-panicled Rush, S2/G5 (Knapp 983 DOV)

Juncus bufonius L., Toad Rush (Knapp 1285 DOV)

Juncus caesariensis Coville, New Jersey Rush, S1/G2G3 (Knapp 195-03 TAWES)

Juncus canadensis J. Gay ex Laharpe, Canada Rush (Knapp 1626 DOV)

Juncus compressus Jacq., Round-fruited Rush, † (Knapp 2007 DOV)

Juncus coriaceus Mack., Leathery Rush (Knapp 578 DOV) [Juncus setaceus Rostk.]

Juncus debilis A. Gray, Weak Rush (Knapp 2593 DOV) [Juncus acuminatus Michx. var. debilis (A. Gray) Engelm.]

Juncus dichotomus Elliott, Forked Rush (Knapp 559 DOV) [Juncus platyphyllus (Wiegand) Fernald]

Juncus diffusissimus Buckley, Diffuse Rush (Knapp 2432 DOV) Juncus dudleyi Wiegand, Dudley's Rush (Boone 850919 DOV)

Juncus effusus L. ssp. solutus (Fernald \& Wiegand) Hämet-Ahti, Soft Rush (Knapp 2256 DOV)

Juncus elliottii Chapm., Elliott's Rush, S1/G4G5 (Knapp 2407 DOV)

- Juncus filiformis L., Thread Rush—see Excluded Flora section. Juncus gerardii Loisel., Saltmarsh Rush (Knapp 1204 DOV)

Juncus longii Fernald, Long's Rush, S1/G3Q (Knapp 1622 DOV)
Juncus marginatus Rostk., Grass-leaved Rush (Knapp 2405 DOV)

Juncus megacephalus M. A. Curtis, Large-headed Rush, S1?/ G4G5 (Knapp 2637 Assateague Island National Seashore Herbarium)

Juncus militaris Bigelow, Bayonet Rush, SH/G4 (Canby s.n. NY)

Juncus pelocarpus E. Mey., Brown-fruited Rush, S1/G5 (Knapp 2258 DOV)

[Juncus abortivus Chapm.]

- Juncus polycephalos Michx., Many-headed Rush-see Excluded Flora section.

[Juncus polycephalus Michx.-—orthographic variant]

Juncus pylaei Laharpe, Common Rush (Knapp 2786 DOV)

Juncus repens Michx., Creeping Rush (Knapp 2595 DOV)

Juncus roemerianus Scheele, Black Needle Rush (Knapp 1691 DOV)

Juncus scirpoides Lam. var. compositus Harper, Lobe-headed Scirpus-Like Rush (Knapp 2649 DOV)

Juncus scirpoides Lam. var. scirpoides, Scirpus-like Rush (Knapp 2610 DOV)

Juncus secundus P. Beauv. ex Poir., Secund Rush (Knapp 1146 DOV)

Juncus subcaudatus (Engelm.) Coville \& S. F. Blake, Woodland Rush (Knapp 985 DOV)

Juncus tenuis Willd., Path Rush (Knapp 564 DOV)

Juncus torreyi Coville, Torrey's Rush, S1/G5 (Knapp 1616 DOV)

Juncus validus Coville, Vigorous Rush, † (Knapp 1550 DOV) [Juncus crassifolius (Michx.) Buchenau]

Luzula acuminata Raf., Hairy Woodrush (Frye 3055 DOV) [Luzula acuminata var. carolinae (S. Watson) Fernald]

Luzula bulbosa (Wood) Rydb., Bulbous Woodrush (Knapp 2384 DOV)

Luzula echinata (Small) F. J. Herm., Spreading Woodrush (Knapp 2373 DOV)

Luzula multiflora (Retz.) Lej. ssp. multiflora, Common Woodrush (Knapp 2402 DOV)

Oreojuncus trifidus (L.) Záv. Drábk. \& Kirschner, Highland Rush, S1/G5 (Riefner 80294 MARY)

[Juncus trifidus L.]

JUNCAGINACEAE

(Arrowgrass Family)

- Triglochin maritima L., Seaside Arrowgrass-see Excluded Flora section.

Triglochin striata Ruiz \& Pav., Southern Arrowgrass, S1/G5 (Hirst \& Wilson 299 DOV)

LAMIACEAE [Labiatae]

(Mint Family)

Agastache nepetoides (L.) Kuntze, Yellow Giant Hyssop (Hirst \&. Wilson 1178 US) 
Agastache scrophulariifolia (Willd.) Kuntze, Purple Giant Hyssop, S1S2/G4 (Baltars $684 \mathrm{MARY})$

Ajuga chamaepitys (L.) Schreb., Yellow Bugle, † (Uhler s.n. US) Ajuga genevensis L., Geneva Bugle, † (Norton s.n. US)

Ajuga reptans L., Carpet Bugle, † (Hochwald s.n. US)

Ballota nigra L., Black Horehound, † (Turoin 258 US)

Blephilia ciliata (L.) Benth., Downy Woodmint, S3S4/G5 (Luttman 643 DOV)

Blephilia hirsuta (Pursh) Benth., Hairy Woodmint, SH/G5 (Frye \& Knapp 5416 DOV)

Callicarpa americana L., French Mulberry, † (Canby \& Rose 839 DOV)

Callicarpa dichotoma (Lour.) K. Koch, Purple Beautyberry, † (Knapp \& Harrison 5319 MARY)

Chaiturus marrubiastrum (L.) Ehrh. ex Rchb., Horehound, $\dagger$ (Penson s.n. MARY)

[Leonurus marrubiastrum L.]

- Clerodendrum japonicum (Thunb.) Sweet, Japanese Glorybower-see Excluded Flora section.

- Clinopodium acinos (L.) Kuntze, Basil Thyme-see Excluded Flora section.

[Acinos arvensis (Lam.) Dandy; Satureja acinos (L.) Scheele]

Clinopodium calamintha (L.) Stace, Lesser Calamint, $\dagger$ (Norton s.n. MARY)

[Clinopodium calamintha var. nepetoides (Jordan) Briquet; C. nepeta (L.) Savi; C. nepeta ssp. glandulosa (Riquien) P. W. Ball; Satureja calamintha (L.) Scheele var. nepeta (L.) Briq.]

Clinopodium vulgare L., Field Basil (McAvoy 5172 DOV)

Collinsonia canadensis L., Canada Horse-balm (Randall 35 US) [Collinsonia scabriuscula Aiton; C. tuberosa Michx.]

Cunila origanoides (L.) Britton, Common Dittany (Isanogle 33 US) [Mappia origanoides (L.) House]

- Dracocephalum parviflorum Nutt., American Dragonheadsee Excluded Flora section.

Galeopsis tetrahit L., Brittle-stem Hempnettle, † (Luttman s.n. DOV)

Glechoma hederacea L., Ground Ivy, † (Uhler s.n. US)

- Hedeoma bispida Pursh, Rough False Pennyroyal-see Excluded Flora section.
Hedeoma pulegioides (L.) Pers., American False Pennyroyal (Hitchcock 15769 US)

Lamiastrum galeobdolon (L.) Ehrend. \& Polatschek, Yellow Archangel, † (Hall JH2014.05.10-01 MARY)

[Lamium galeobdolon (L.) L.]

Lamium album L., White Deadnettle, † (Fordman 134 MARY)

Lamium amplexicaule L., Common Deadnettle, † (Fleming 2022 US)

Lamium maculatum L., Spotted Deadnettle, † (Knapp 520 DOV)

Lamium purpureum L., Purple Deadnettle, † (Longbottom 12479 US)

Leonurus cardiaca L., Common Motherwort, † (Windler \& Keenan 3125 DOV)

Lycopus americanus Muhl. ex Bartram, American Bugleweed (Seyfried \& Outten CEP86-765 DOV)

Lycopus amplectens Raf., Sessile-leaf Bugleweed, S1/G5 (Boone s.n. TAWES)

[Lycopus pubens Britton; L. sessilifolius A. Gray]

Lycopus europaeus L., European Bugleweed, † (Leonard 5695 US)

Lycopus rubellus Moench, Taperleaf Bugleweed (Longbottom 10758 DOV)

[Lycopus velutinus Rydb.]

Lycopus uniflorus Michx., Northern Bugleweed (Leonard 21703 US)

Lycopus virginicus L., Virginia Bugleweed (Knapp 1029 DOV)

Marrubium vulgare L., Common Horehound, † (Leonard 21898 US)

Melissa officinalis L., Garden Balm, † (Roller 793 US)

Mentha aquatica L., Water Mint, † (Longbottom 12061 DOV)

Mentha arvensis L., Wild Mint, † (Hotchkiss 5371 US) [Mentha gentilis L.]

Mentha canadensis L., Canada Mint (Tucker s.n. DOV)

Mentha longifolia (L.) Huds., Longleaf Spearmint, $†$ (Steele s.n. US)

Mentha pulegium L., Pennyroyal, † (Norton s.n. US)

Mentha spicata L., Spearmint, † (Longbottom 10040 DOV) [Mentha sylvestris L.]

Mentha suaveolens Ehrh., Apple Mint, † (Longbottom et al. 3321 MARY)

Monarda clinopodia L., Basil Beebalm, S3S4/G5 (Driskill et al. CEP85-468 DOV)

Monarda didyma L., Oswego-tea (Morton $2078 b$ US)

Monarda fistulosa L. var. fistulosa, Wild Bergamot (Burnett s.n. MARY) 
Monarda fistulosa L. var. mollis (L.) Benth., Soft Wild Bergamot (Luttman s.n. DOV)

[Monarda mollis L.; M. scabra Beck]

Monarda media Willd., Purple Bergamot, †? SH/G4? (Hill \& Cress 11815 MARY)

Monarda punctata L. var. punctata, Spotted Beebalm (Knapp 121-03 US)

Mosla scabra (Thunb.) C. Y. Wu \& H. W. Li, Rough Mosla, † (Johnson s.n. NY)-perhaps a waif, but its recent detection (late 2018) makes its status uncertain

Nepeta cataria L., Catnip, † (Killip 13225 US)

Origanum vulgare L., Wild Marjoram, † (Baltars 682 US)

Perilla frutescens (L.) Britton, Beefsteak Plant, $\ddagger$ (Driskill et al. CEP-2137 DOV)

[Perilla frutescens var. crispa W. Deane]

- Physostegia purpurea (Walter) S. F. Blake, Eastern False Dragonhead-see Excluded Flora section.

Physostegia virginiana (L.) Benth. ssp. virginiana, False Dragonhead (Longbottom et al. 7766 DOV)

[Dracocephalum denticulatum Aiton; D. virginianum L.]

Prunella laciniata (L.) L., Cutleaf Self-heal, † (Sargent s.n. US)

Prunella vulgaris L. ssp. lanceolata (W. P. C. Barton) Hultén, American Self-heal (Driskill et al. CEP85-1103 DOV)

Prunella vulgaris L. ssp. vulgaris, Eurasian Self-heal, $†$ (Freeman s.n. NA)

Pycnanthemum clinopodioides Torr. \& A. Gray, Basil Mountainmint, SH/G1G2 (Steele s.n. US)

- Pycnanthemum flexuosum (Walter) Britton, Sterns, \& Poggenb., Appalachian Mountainmint-see Excluded Flora section.

Pycnanthemum incanum (L.) Michx., Hoary Mountainmint (Frye GRSFBD 669 TAWES)

[Pycnanthemum incanum var. puberulum (E. Grant \& Epling) Fernald]

Pycnanthemum muticum (Michx.) Pers., Blunt Mountainmint (Steury 980821.1 US)

- Pycnanthemum pycnanthemoides Fernald, Southern Mountainmint, SH/G5-see Excluded Flora section.

Pycnanthemum setosum Nutt., Awned Mountainmint (Knapp 577 DOV)

Pycnanthemum tenuifolium Schrad., Slender Mountainmint (Naczi 8962 DOV)

Pycnanthemum torreyi Benth., Torrey's Mountainmint, S1/ G2 (Fleming 970904 TAWES)

Pycnanthemum verticillatum (Michx.) Pers., Whorled Mountainmint, S1/G5 (McAvoy $1756 \mathrm{DOV})$

[Pycnanthemum pilosum Nutt.; P. verticillatum var. pilosum (Nutt.) Cooperr.]
Pycnanthemum virginianum (L.) B. L. Rob. \& Fernald, Virginia Mountainmint, S2/G5 (Fleming 970819 TAWES)

Salvia lyrata L., Lyreleaf Sage (Longbottom 7287 \& Rowan DOV)

Salvia officinalis L., Kitchen Sage, † (Ditman s.n. MARY)

Salvia pratensis L., Meadow Sage, † (Norton s.n. MARY)

Salvia reflexa Hornem., Lanceleaf Sage, $\dagger$ (Petrak s.n. US)

Salvia urticifolia L., Nettleleaf Sage, SX/G5 (Sherard s.n. OXF) -not seen since the colonial era; see Brown et al. (1987).

Salvia verbenacea L., Verbena Sage, † (Arsène 225 US)

- Salvia verticillata L., Lilac Sage-see Excluded Flora section.

Scutellaria elliptica Muhl. var. elliptica, Hairy Skullcap (Knapp 2335 DOV)

[Scutellaria ovalifolia Pers.; S. pilosa Michx.]

Scutellaria galericulata L., Hooded Skullcap, S2/G5 (Steury 020713.1 US)

Scutellaria incana Biehler var. incana, Hoary Skullcap, S3/ G5T5 (Grund et al. 4686 CM)

Scutellaria integrifolia L., Hyssop Skullcap (Naczi 8957 DOV)

Scutellaria lateriflora L., Mad-dog Skullcap (Driskill et al. CEP85-1318 DOV)

Scutellaria leonardii Epling, Shale Barren Skullcap, S2/G4 (Frye \& Knapp 5943 TAWES)

[Scutellaria ambigua Nutt.; S. parvula Michx. var. missouriensis (Torr.) Goodman \& C. A. Lawson]

Scutellaria nervosa Pursh, Veined Skullcap, S1S2/G5 (Holt \& Ebert s.n. TAWES)

[Scutellaria cordifolia Muhl.]

Scutellaria ovata Hill ssp. rugosa (Alph. Wood) Epling, Rough Heartleaf Skullcap, S3/G5TNR (Knapp 3360 NCU) [Scutellaria ovata Hill ssp. virginiana Epling]

- Scutellaria parvula Michx. var. parvula, Small Skullcap-see Excluded Flora section.

Scutellaria saxatilis Riddell, Rock Skullcap, S1/G3 (Terrell 4255 US)

Scutellaria serrata S. Andrews, Showy Skullcap, S3/G4G5 (Maxon 6112 US)

Stachys aspera Michx., Gritty Hedge-nettle, S1/G4? (Hirst $1150 \mathrm{DOV})$

[Stachys ambigua (Gray) Britton; S. hyssopifolia Michx. Var. ambigua Gray]

Stachys byzantina K. Koch, Wooly Hedge-nettle † (Steury 980515.9 US)

- Stachys clingmanii Small, Clingman's Hedge-nettle-see Excluded Flora section.

- Stachys cordata Riddell, Heartleaf Hedge-nettle-see Excluded Flora section. [Stachys riddellii House]

Stachys eplingii J. B. Nelson, Epling's Hedge-nettle, S1/G1G2 (Wiegand s.n. TAWES)

Stachys bispida Pursh, Hispid Hedge-nettle (Knapp 1556b DOV) 
Stachys byssopifolia Michx. var. byssopifolia, Hyssopleaf Hedge-nettle, S1/G4G5 (Plitt s.n. US)

- Stachys latidens Small ex Britton, Broad-Tooth Hedgenettle-see Excluded Flora section.

- Stachys longispicata Boiss., Longspike Hedge-nettle, † Waif Reed (1964)

- Stachys nuttallii Shuttlew. ex Benth., Nuttall's Hedge-nettlesee Excluded Flora section.

Stachys palustris L., Marsh Hedge-nettle, † (Steury 050625.1 US)

- Stachys pilosa Nutt., Hairy Hedge-nettle-see Excluded Flora section.

Stachys tenuifolia Willd., Smooth Hedge-nettle (Steury 980807.6 US)

[Stachys tenuifolia var. perlonga Fernald]

Teucrium canadense L., American Germander (Redmond 179 DOV)

[Teucrium canadense var. occidentale (A. Gray) E. C. McClint. \& Epling; T. canadense var. virginicum (L.) Eaton; T. littorale E. P. Bicknell; T. occidentale A. Gray]

- Teucrium laciniatum Torr., Cutleaf Germander, † Waif Reed (1964)

- Thymus praecox Opiz ssp. arcticus (Durand) Jalas, Mother-ofthyme-see Excluded Flora section.

Thymus pulegioides L., Lemon Thyme, † (Topping 3308 US) [Thymus serpyllum L.]

Trichostema brachiatum L., False Pennyroyal, S3/G5 (Lea 3283 DOV)

[Isanthus brachiatus (L.) Britton, Sterns, \& Poggenb.]

Trichostema dichotomum L., Forked Bluecurls (Knapp 1596 DOV)

Trichostema setaceum Houtt., Narrowleaf Bluecurls, S1/G5 (Knapp \& Thompson 3361 TAWES)

[Trichostema dichotomum L. var. lineare (Walter) Pursh; T. lineare Walter]

- Vitex agnus-castus L., Lilac Chaste-tree-see Excluded Flora section.

- Vitex negundo L., Negundo Chaste-tree-see Excluded Flora section.

[Vitex negundo var. heterophylla (Franch.) Rehder]

Vitex rotundifolia L. f., Roundleaf Chaste-tree $\ddagger$ (Chase s.n. Assateague Island National Seashore Herbarium)

\section{LARDIZABALACEAE (Lardizabala Family)}

Akebia quinata (Houtt.) Decne., Five-leaf Akebia, ‡ (Mora 45 MARY)

\section{LAURACEAE}

(Laurel Family)

Lindera benzoin (L.) Blume, Northern Spicebush (Naczi 8057 DOV)

[Lindera benzoin var. pubescens (E. J. Palmer \& Steyerm.) Rehder]
Litsea aestivalis (L.) Fernald, Pondspice, S1/G3? (Knapp \& Frye s.n. TAWES)

Persea palustris Sarg., Red Bay, S1/G5 (Knapp et al. 08-01 TAWES)

[Persea pubescens (Pursh) Sarg.; Tamala pubescens (Pursh) Small]

Sassafras albidum (Nutt.) Nees, Sassafras (Hotchkiss 7209 US) [Sassafras albidum var. molle (Raf.) Fernald]

LENTIBULARIACEAE (Bladderwort Family)

Utricularia cornuta Michx., Horned Bladderwort, S1/G5 (Stango et al. 2010132-1 TAWES)

Utricularia geminiscapa Benj., Hidden-fruited Bladderwort (Hotchkiss 8020 US)

Utricularia gibba L., Humped Bladderwort (Fosberg 33439 US) [Utricularia biflora Lam.; U. pumila Walter]

Utricularia inflata Walter, Swollen Bladderwort, S2/G5 (Reveal et al. 7107 MARY)

Utricularia intermedia Hayne, Flatleaved Bladderwort, S1/ G5 (Tyndall 825942 DOV)

Utricularia juncea Vahl, Southern Bladderwort (Walker 7372 US)

Utricularia macrorhiza Leconte ex Torr, Greater Bladderwort (Hall JH2014.08.14-18 MARY)

[Utricularia vulgaris L. ssp. macrorhiza (Leconte ex Torr.) R. T. Clausen]

Utricularia purpurea Walter, Purple Bladderwort, S1/G5 (Boone s.n. TAWES)

Utricularia radiata Small, Small Swollen Bladderwort (McAvoy 1517 DOV)

Utricularia resupinata B. D. Greene ex Bigelow, Northeastern Bladderwort, S1/G4 (Naczi \& Davis 602 DOV)

Utricularia striata Leconte ex Torr., Striped Bladderwort, S1/ G4G5 (Sipple 1987 DOV)

[Utricularia fibrosa Britton]

Utricularia subulata L., Zigzag Bladderwort, S3/G5 (Walker 7372 US)

LILIACEAE

(Lily Family)

- Cardiocrinum cordatum Makino, Himalayan Lily, † Waif (Redman 6312 BALT)

Clintonia alleghaniensis Harned, Allegheny Clintonia, S1/ G1Q (Brown s.n. MARY)

Clintonia borealis (Aiton) Raf., Blue Clintonia, S2/G5 (Reveal et al. 5283 MARY)

Clintonia umbellulata (Michx.) Morong, White Clintonia (Brown s.n. MARY)

Erythronium albidum Nutt., White Trout Lily, S2/G5 (Beyersdorfer 27 US) 
Erythronium americanum Ker, Yellow Trout Lily (Knapp 2392 TAWES) [Erythronium americanum ssp. harperi (W. Wolf) C. R. Parks \& Hardin]

- Erythronium umbilicatum C. R. Parks \& Hardin, Dimpled Trout Lily—see Excluded Flora section.

Lilium canadense L., Canada Lily (Steele s.n. US) [Lilium canadense var. editorum Fernald]

- Lilium grayi Watson, Gray's Lily—see Excluded Flora section. Lilium lancifolium Thunb., Tiger Lily, † (Windler et al. s.n. NCU) [Lilium tigrinum L.]

Lilium philadelphicum L., Wood Lily, SH/G5 (Shriver s.n. MARY)

Lilium superbum L., Turk's-cap Lily (Chase 7571 US)

Medeola virginiana L., Indian Cucumber (Hotchkiss 5138 US)

Prosartes lanuginosa (Michx.) D. Don, Yellow Fairybells (Williams et al. 1963 MARY)

[Disporum lanuginosum (Michx.) G. Nicholson]

- Prosartes maculata (Buckley) Britton, Yellow Mandarin-see Excluded Flora section.

[Disporum maculatum (Buckley) Britton]

- Streptopus amplexifolius (L.) DC. var. americanus (Schult.) \& Schult. f., Claspleaf Twisted Stalk-see Excluded Flora section.

Streptopus lanceolatus (Aiton) Reveal var. lanceolatus, Twisted Stalk, S1S2/G5T5 (Boone 840720 TAWES)

[Streptopus roseus Michx.]

Tulipa sylvestris L., Woodland Tulip, † (Hill 12687 MARY)

\section{LIMNANTHACEAE (False-mermaid Family)}

Floerkea proserpinacoides Willd., False Mermaidweed (Steury 040501.1 US)

\section{LINACEAE}

(Flax Family)

- Linum floridanum (Planch.) Trel. var. floridanum, Florida Yellow Flax-see Excluded Flora section.

Linum intercursum E. P. Bicknell, Sandplain Flax, S2/G4 (Steury 000826.3 US)

[Cathartolinum intercursum (E. P. Bicknell) Small]

Linum medium (Planch.) Britton var. texanum (Planch.) Fernald, Texas Stiff Yellow Flax (Blake s.n. US)

[Cathartolinum medium (Planch.) Small; Linum virginianum L. var. medium Planch.]

Linum striatum Walter, Ridged Yellow Flax (Strong et al. 1808 US) [Cathartolinum striatum (Walter) Small]

Linum sulcatum Riddell, Grooved Yellow Flax, S1/G5 (Isanogle 2 US)

[Cathartolinum sulcatum (Riddell) Small]
Linum usitatissimum L., Common Flax, † (Fosberg 66266 US)

Linum virginianum L., Virginia Flax (Hotchkiss 5667 US)

[Cathartolinum virginianum (L.) Rchb.]

\section{LINDERNIACEAE}

(False Pimpernel Family)

Lindernia dubia (L.) Pennell, Yellowseed False Pimpernel (Norton s.n. MARY)

[Lindernia anagallidea (Michx.) Pennell; L. dubia var. anagallidea (Michx.) Cooperr.]

Micranthemum micranthemoides (Nutt.) Wettst., Nuttall's Micranthemum, SH/GH Presumed Extinct (Canby s.n. DOV)

[Hemianthus micranthemoides Nutt.]

LOGANIACEAE

(Logania Family)

- Spigelia marilandica L., Woodland Pinkroot-see Excluded Flora section.

[Spigelia corymbosa Raf.]

LYTHRACEAE

(Loosestrife Family)

Ammannia coccinea Rottb., Valley Redstem (Fosberg \& Rainey 60658 US)

Ammannia latifolia L., Koehne Ammannia, S2/G5 (Steury 902.1 US)

[Ammannia koehnei Britton]

Cuphea viscosissima Jacq., Blue Waxweed (Hitchcock 15714 US) [Cuphea petiolata (L.) Koehne; Parsonsia petiolata (L.) Rusby]

Decodon verticillatus (L.) Elliott, Hairy Swamp Loosestrife (Cooley s.n. TAWES)

Lagerstroemia indica L., Crape Myrtle, † (Quinn s.n. MARY)

Lythrum alatum Pursh, Winged Lythrum, S1/G5 (Killip 31898 US)

Lythrum hyssopifolia L., Hyssop Loosestrife, † (Hotchkiss 7736 US)

Lythrum lineare L., Narrow Loosestrife (Shull 331 US)

Lythrum salicaria L., Purple Loosestrife, $\ddagger$ (Cooley s.n. TAWES)

Rotala ramosior (L.) Koehne, Lowland Rotala (Naczi 8990 DOV)

Trapa natans L., Water Chestnut, $\ddagger$ (Hermann 9598 US)

MAGNOLIACEAE (Magnolia Family)

Liriodendron tulipifera L., Tuliptree (Coville 900 US)

Magnolia acuminata L., Cucumber Magnolia (Hickey II 611 BALT) 
[Magnolia acuminata var. cordata (Michx.) Sarg.; Yulania acuminata (L.)D. L. Fu]

Magnolia grandiflora L., Large-flowered Magnolia, † (Keene 11 BALT)

[Magnolia foetida (L.) Sarg.]

Magnolia macrophylla Michx., Bigleaf Magnolia, † (Guerke s.n. BALT)

[Metamagnolia macrophylla (Michx.) Sima \& S. G. Lu]

Magnolia tripetala L., Umbrella Magnolia, S3/G5 (Boone s.n. TAWES)

[Houpoea tripetala (L.) Sima \& S. G. Lu]

Magnolia virginiana L., Sweetbay Magnolia (Beyersdorfer 112 US)

MALVACEAE

(Mallow Family)

- Abelmoschus esculentus (L.) Moench, Okra, † Waif (Coulter s.n. US)

Abutilon theophrasti Medik., Velvetleaf, $\dagger$ (Frye s.n. TAWES) [Abutilon abutilon (L.) Rusby]

Alcea rugosa Alef., Hairy Hollyhock, † (Hill 10372 MARY) [Alcea ficifolia L.]

Alcea rosea L., Hollyhock, † (Hill 10371a MARY) [Althaea rosea (L.) Cav.]

- Althaea officinalis L., Common Marshmallow-see Excluded Flora section.

Anoda cristata (L.) Schltdl., Spurred Anoda, † (Ebert \& Holt $126 \mathrm{EH} \mathrm{DOV})$

- Firmiana simplex W. Wright, Chinese Parasol Tree-see Excluded Flora section.

[Firmiana platanifolia (L. f.) Schott \& Endl.; Sterculia platanifolia L. f.]

- Gossypium hirsutum L., Upland Cotton, † Waif Reed (1964) [Gossypium herbaceum L.]

Hibiscus laevis All., Halberd-leaf Rose Mallow, S3/G5 (McCarthy s.n. TAWES)

[Hibiscus militaris Cav.]

Hibiscus moscheutos L., Swamp Rose Mallow (Hitchcock 15729 US)

[Hibiscus incanus J. C. Wendl.; H. moscheutos var. incanus (J. C. Wendl.) H. E. Ahles; H. moscheutos var. palustris (L.) R. T. Clausen; H. oculiroseus Britton]

Hibiscus syriacus L., Rose of Sharon, † (Duvall 176 BALT)

Hibiscus trionum L., Flower of an Hour, † (Fosberg 39882 US)

Kosteletzkya pentacarpos (L.) Ledeb., Virginia Seashore Mallow (McGowan s.n. BALT)

[Kosteletzkya althaeifolia (Chapm.) Rusby; K. virginica (L.) C. Presl ex A. Gray]
- Lavatera trimestris L., Annual Mallow-see Excluded Flora section.

Malva moschata L., Musk Cheeseweed, † (Wolf 105 BALT)

Malva neglecta Wallr., Common Mallow, † (Hotchkiss 5506 US) [Malva rotundifolia $\mathrm{L}$.

- Malva parviflora L., Small Whorled Cheeseweed, † Waif Reed (1964)

- Malva pusilla Sm., Low Mallow-see Excluded Flora section. Malva sylvestris L., High Cheeseweed, † (Norton s.n. MARY)

- Malva verticillata L., Curled Cheeseweed-see Excluded Flora section.

- Melochia corchorifolia L., Chocolateweed, † Waif Reed (1964)

Modiola caroliniana (L.) G. Don, Carolina Bristle Mallow, † (Davis 7241 Patuxent River Naval Air Station)

Napaea dioica L., Glade Mallow, S1/G4 (Lea 418 TAWES)

Ripariosida hermaphrodita (L.) Weakley \& D. B. Poind., Virginia Mallow, S1/G3 (Holt \& Ebert s.n. TAWES) [Sida hermaphrodita (L.) Rusby]

- Sida cordata (Burm. f.) Borss. Waalk., Heartleaf Fanpetals, † Waif Reed (1964)

[Sida humilis Willd. var. veronicaefolia Lam.]

Sida rhombifolia L., Cuban Jute, † (Stasz Photo Voucher MPA)

Sida spinosa L., Prickly Mallow, † (Knapp \& Longbottom 1583 TAWES)

[Sida angustifolia Lam.]

- Sphaeralcea angustifolia G. Don, Narrowleaf Globemallow, † Waif Reed (1964)

Tilia americana L., American Basswood (Engh s.n. MARY) [Tilia americana var. heterophylla (Vent.) Loudon; T. australis Small; T. caroliniana Mill.; T. eburnea Ashe; T. floridana Small; T. heterophylla Vent.; T. leucocarpa Ashe; T. michauxii Nutt.]

Tilia cordata Mill., Littleleaf Linden, † (Reveal \& Broome 6167 MARY)

MARTYNIACEAE

(Martynia Family)

- Proboscidea louisianica (Mill.) Thell., Louisiana Unicornplant, † Waif (Norton s.n. MARY)

[Martynia louisianica Mill.]

MAZACEAE

(Mazus Family)

Mazus miquelii Makino, Miquel's Mazus, † (Seymour 16608 $\mathrm{MO})$

Mazus pumilus (Burm. f.) Steenis, Japanese Mazus, † (Hambleton 208 US)

[Mazus japonicus (Thunb.) Kuntze] 


\section{MELANTHIACEAE}

(Bunchflower Family)

Amianthium muscitoxicum (Walter) A. Gray, Fly Poison, - S2/G4G5 (Arsène 469 US)

Chamaelirium luteum (L.) A. Gray, Fairywand, S2/G5 (Fessenden 5417 US)

Helonias bullata L., Swamp Pink, S2/G3, LT (Walker 7379 US)

Stenanthium gramineum (Ker Gawl.) Morong, Eastern Featherbells, S1/G4G5 (Lehtonen \& Young 50 US)

[Stenanthium gramineum var. micranthum Fernald; S. gramineum var. robustum (S. Watson) Fernald; S. robustum S. Watson]

Stenanthium leimanthoides (A. Gray) Zomlefer \& Judd, Pine Barrens Deathcamus, S1/G4Q (Knapp et al. 2968 US)

[Zigadenus leimanthoides A. Gray]

Trillium cernuum L., Nodding Trillium, S3/G5 (Fessenden s.n. US)

[Trillium cernuum var. macranthum A. J. Eames \& Wiegand]

Trillium cuneatum Raf., Little Sweet Betsy, † (Harmeyer s.n. MARY)

Trillium erectum L., Red Trillium (Samm s.n. MARY)

Trillium flexipes Raf., Nodding Wakerobin, S1/G5 (Baltars 703 US)

Trillium grandiflorum (Michx.) Salisb., White Trillium (Walker s.n. MARY)

Trillium luteum (Muhl.) Harb., Yellow Trillium, † (Steury 990427.1 US)

[Trillium viride L. C. Beck var. luteum (Muhl.) Gleason]

Trillium nivale Riddell, Snow Trillium, S1/G4 (Reveal et al. 5303 MARY)

Trillium pusillum Michx. var. virginianum Fernald, Virginia Wakerobin, S2/G3T2 (Naczi 9516 DOV)

Trillium sessile L., Toadshade (Hill 9810 MARY)

Trillium undulatum Willd., Painted Trillium (Reveal et al. 5341 MARY)

Veratrum bybridum (Walter) J. H. Zimmerman ex Zomlefer, Slender Bunchflower, S1/G5 (Freeman s.n. NA)

[Melanthium bybridum Walter; M. latifolium Desr.; Veratrum latifolium (Desr.) Zomlefer]

Veratrum parviflorum Michx., Appalachian Bunchflower (Wiegand Photo Voucher TAWES)

[Melanthium parviflorum (Michx.) S. Watson]

Veratrum virginicum (L.) W. T. Aiton, Virginia Bunchflower, - S3/G5 (McAvoy 4437 DOV)

[Melanthium virginicum L.]
Veratrum viride Aiton var. viride, Green False Hellebore (Maxon 6448 US)

- Xerophyllum asphodeloides (L.) Nutt., Eastern Turkeybeardsee Excluded Flora section.

MELASTOMATACEAE

(Melastome Family)

- Rhexia aristosa Britton, Awnpetal Meadow-beauty-see Excluded Flora section.

Rhexia mariana L., Maryland Meadow-beauty (Walker 1110 MARY)

[Rhexia lanceolata Walter]

- Rhexia nashii Small, Nash's Meadow-beauty-see Excluded Flora section.

- Rhexia petiolata Walter, Ciliate Meadow-beauty-see Excluded Flora section.

Rhexia ventricosa Fernald \& Griscom, Swollen Meadow-beauty (Frye 697 TAWES)

[Rhexia mariana L. var. ventricosa (Fernald \& Griscom) Kral \& Bostich]

Rhexia virginica L., Virginia Meadow-beauty (Strong \& Simmons 1974 US)

[Rhexia stricta Pursh; R. virginica var. purshii (Spreng.) C. W. James]

MELIACEAE

(Mahogany Family)

- Melia azedarach L., Chinaberry, † Waif (Hill \& Riefner 10825 MARY)

- Toona ciliata Roem., Australian Red Cedar-see Excluded Flora section.

Toona sinensis (A. Juss.) M. Roem., Chinese Mahogany, † (Kyde s.n. TAWES)

MENISPERMACEAE (Moonseed Family)

Menispermum canadense L., Canada Moonseed (Hitchcock 12754 US)

MENYANTHACEAE (Buckbean Family)

Menyanthes trifoliata L., Bog Buckbean, S1/G5 (Boone s.n. TAWES)

Nymphoides aquatica (J. F. Gmel.) Kuntze, Big Floatingheart, - S1/G5 (Sipple 1983 DOV)

Nymphoides cordata (Elliott) Fernald, Little Floatingheart, - S1/G5 (Norton s.n. MARY)

[Nymphoides lacunosa (Vent.) Kuntze]

Nymphoides peltata (S. G. Gmel.) Kuntze, Yellow Floatingheart, † (Ferraro Photo Voucher MPA) 
MOLLUGINACEAE

(Carpetweed Family)

- Mollugo gracillima Andersson, Slender Carpetweed, † Waif Reed (1964)

- Mollugo nudicaulis Lam., Naked-stem Carpetweed, † Waif Reed (1964)

Mollugo verticillata L., Green Carpetweed, † (Boone s.n. TAWES)

\section{MONTIACEAE}

(Montia Family)

Claytonia caroliniana Michx., Carolina Springbeauty, S3/G5 (Steury 010410.3 US)

Claytonia virginica L., Narrowleaf Springbeauty (Chase 6863 US)

[Claytonia media (DC.) Link; C. virginica var. acutiflora DC.]

Montia linearis (Douglas) Greene, Narrowleaf Miners-lettuce, † (Knapp 2672 DOV)

\section{MORACEAE}

(Mulberry Family)

Broussonetia papyrifera (L.) Vent., Paper Mulberry, † (Leonard s.n. US)

[Papyrius papyriferus (L.) Kuntze]

Fatoua villosa (Thunb.) Nakai, Hairy Crabweed, † (Steury \& Saffer 990800 US)

- Ficus carica L., Edible Fig, † Waif. Not established and rarely persisting after cultivation (Worthley s.n. MARY)

Maclura pomifera (Raf.) C. K. Schneid., Osage-orange, † (Smith 5034 US)

[Toxylon pomiferum Raf.]

Morus alba L., White Mulberry, $\ddagger$ (Hotchkiss \& Leonard 3357 US)

Morus rubra L., Red Mulberry (McAvoy 3366 DOV)

\section{MYRICACEAE (Bayberry Family)}

Comptonia peregrina (L.) J. M. Coult., Sweet-fern (Knapp 2628 DOV)

Morella caroliniensis (Mill.) Small, Evergreen Bayberry, S1/ G5 (Knapp 2653 TAWES)

[Myrica caroliniensis Mill.; M. heterophylla Raf.]

Morella cerifera (L.) Small, Southern Bayberry (Knapp 2662 DOV)

[Myrica cerifera L.]

Morella pensylvanica (Mirbel) Kartesz, Northern Bayberry (Knapp 2981 TAWES)

[Myrica pensylvanica Mirbel]
NARTHECIACEAE

(Bog Asphodel Family)

Aletris aurea Walter, Golden Colicroot, SH/G5 (McAtee 2909 US)

Aletris farinosa L., White Colicroot (McAvoy 1811 DOV)

- Narthecium americanum Ker Gawl., Yellow Asphodel, † Waif intentionally introduced at a single site, Prince George's County; this planting persisted for a few years but is not considered established in the state (Walker 1330 MARY)

NELUMBONACEAE

(Lotus Lily Family)

Nelumbo lutea (Willd.) Pers., American Lotus (Uhler s.n. BALT)

- Nelumbo nucifera Gaertn., Sacred Lotus-see Excluded Flora section

NYCTAGINACEAE

(Four-o'clock Family)

- Boerhavia coccinea Mill., Scarlet Spiderling, † Waif Reed (1964)

- Boerhavia erecta L., Erect Spiderling, † Waif Reed (1964)

- Mirabilis jalapa L., Common Four-o'clock, † Waif (Bitman s.n. MARY)

Mirabilis nyctaginea (Michx.) MacMill., Wild Four-o'clock (Steele s.n. US)

[Allionia nyctaginea Michx.]

NYMPHAEACEAE

(Water Lily Family)

Nuphar advena (Aiton) W. T. Aiton, Broadleaf Pond Lily (Uhler 102 BALT)

[Nuphar lutea (L.) Sm. ssp. advena (Aiton) Kartesz \& Gandhi]

Nuphar variegata Engelm. ex Durand, Yellow Cow Lily (Hall Photo Voucher MPA)

[Nuphar lutea (L.) Sm. ssp. variegata (Engelm. ex Durand) E. O. Beal]

Nymphaea odorata Aiton ssp. odorata, American Water Lily (Marshall s.n. US)

[Castalia lekophylla Small; C. minor (Sims) Nyár.; C. odorata (Aiton) Wood; C. odorata var. rosea (Pursh) Britton]

- Nymphaea odorata Aiton ssp. tuberosa (Paine) Wiersema \& Hellquist, American White Water Lily-see Excluded Flora section.

[Nymphaea tuberosa Paine]

NYSSACEAE

(Tupelo Family)

Nyssa biflora Walter, Swamp Blackgum (Naczi 11577 DOV)

[Nyssa sylvatica Marshall var. biflora (Walter) Sarg.]

Nyssa sylvatica Marshall, Blackgum (Knapp 2647 DOV)

OLEACEAE

(Olive Family)

Chionanthus virginicus L., Fringetree (Coville s.n. US) 
Forsythia suspensa (Thunb.) Vahl, Weeping Forsythia, † (Kress et al. 89-2680 US)

Forsythia viridissima Lindl., Greenstem Forsythia, † (Longbottom 10923 DOV)

Fraxinus americana L., White Ash (Hotchkiss \& Leonard 3572 US)

[Fraxinus americana var. curtissii Sudw.]

Fraxinus biltmoreana Beadle, Biltmore Ash (Lamb s.n. US)

Fraxinus caroliniana Mill., Carolina Ash, S2/G4G5 (Knapp 4030 TAWES)

Fraxinus nigra Marshall, Black Ash, S3/G5 (Knapp 2180 DOV)

Fraxinus pennsylvanica Marshall, Green Ash (Hitchcock 12963 US)

[Fraxinus darlingtonii Britton; F. lanceolata Borkh.; F. pennsylvanica var. subintegerrima (Vahl) Fernald; F. smallii Britton]

Fraxinus profunda (Bush) Bush, Pumpkin Ash (Knapp 2644 DOV)

[Fraxinus michauxii Britton]

- Jasminum nudiflorum Lindl., Winter Jasmine-see Excluded Flora section.

Ligustrum japonicum Thunb., Japanese Privet, $\ddagger$ (Knapp 2944 DOV)

- Ligustrum lucidum Aiton, Glossy Privet, † Waif (Ditman s.n. MARY) specimens may have been from cultivation

Ligustrum obtusifolium Siebold \& Zucc. var. obtusifolium, Border Privet, † (Harms 6564 MARY)

[Ligustrum amurense Carrière; L. obtusifolium var. regelianum Rehder; L. obtusifolium var. suave (Kitag.) H. Hara.]

Ligustrum ovalifolium Hassk., California Privet, † (Hill 10399 MARY)

Ligustrum quihoui Carrière, Waxyleaf Privet, † (Hill s.n. NCU)

Ligustrum sinense Lour., Chinese Privet, ‡ (Longbottom 11544 DOV)

Ligustrum vulgare L., European Privet, $\ddagger$ (Longbottom 1364 DOV)

Osmanthus heterophyllus (G. Don) P. S. Green, Holly Osmanthus, $†$ (Rudder Photo Voucher MPA)

Syringa vulgaris L., Common Lilac, † (Cohen 21 MARY)perhaps not truly escaped and merely long persistent after cultivation

\section{ONAGRACEAE}

(Evening-primrose Family)

Chamaenerion angustifolium (L.) Holub, Fireweed (McAtee 2905 US)

[Chamerion angustifolium (L.) Holub; Epilobium angustifolium L.]
Circaea alpina L., Small Enchanter's Nightshade (Hubick Photo Voucher MPA)

Circaea canadensis (L.) Hill ssp. canadensis, Southern Broadleaf Enchanter's Nightshade (Fosberg 56252 US)

[Circaea latifolia Hill; C. lutetiana (L.) Asch. \& Magnus; C. quadrisulcata (Maxim.) Franch. \& Sav.]

Epilobium ciliatum Raf., Hairy Willowherb, S3S4/G5 (Simmons \& Strong 1820 US)

[Epilobium glandulosum Lehm.]

Epilobium coloratum Biehler, Purpleleaf Willowherb (Hitchcock 15704 US)

Epilobium hirsutum L., Great Hairy Willowherb, † (Baltars 635 US)

Epilobium leptophyllum Raf., Linear-leaf Willowherb, S2S3/ G5 (Baltars 2732 US)

Epilobium parviflorum Schreb., Smallflower Hairy Willowherb, † (Cusick $35719 \mathrm{CM})$

- Epilobium strictum Muhl. ex Spreng., Downy Willowherbsee Excluded Flora section.

Gaura biennis L., Biennial Gaura (Shreve 995 \& Jones US)

Gaura longiflora Spach, Long-flowered Gaura (Hotchkiss s.n. US) [Gaura filiformis Small]

Ludwigia alternifolia L., Bushy Seedbox (Bell et al. 2 US) [Ludwigia alternifolia var. linearifolia Britton]

- Ludwigia brevipes (Long) Eames, Long Beach Seedbox-see Excluded Flora section.

Ludwigia decurrens Walter, Primrose-willow, S2S3/G5 (McCarthy \& Wiegand s.n. TAWES)

[Jussiaea decurrens (Walter) DC.]

Ludwigia glandulosa Walter, Cylindric-fruited Seedbox, S1/ G5 (Knapp 2029 DOV)

Ludwigia hexapetala (Hook. \& Arn.) Zardini, H. Y. Gu \& P. H. Raven, Large-flowered Water-primrose † (Longbottom $15733 \mathrm{NY}$ )

Ludwigia hirtella Raf., Rafinesque's Seedbox, S1/G5 (Boone s.n. TAWES)

Ludwigia leptocarpa (Nutt.) H. Hara, River Seedbox, † (McAvoy 2042 DOV)

[Jussiaea leptocarpa Nutt.; J. pilosa Pohl ex Micheli]

Ludwigia linearis Walter, Narrowleaved Seedbox (Knapp 173-03 TAWES)

Ludwigia palustris (L.) Elliott, Marsh Seedbox (Frye 5123 TAWES)

[Isnardia palustris L.]

Ludwigia peploides (Kunth) P. H. Raven var. glabrescens (Kuntze) Shinners, Floating Seedbox, † (Frye \& Smith s.n. TAWES)

[Jussiaea diffusa Forssk.; J. repens L.; Ludwigia peploides ssp. glabrescens (Kuntze) P. H. Raven]

Ludwigia sphaerocarpa Elliott, Globe-fruited False Loosestrife (Knapp et al. 183-03 TAWES) 
Ludwigia virgata Michx., Savannah Primrose-willow, (Sherard s.n. OXF)-Reported by Reveal et al. (1987), based on a single specimen from the 1700 s whose Maryland provenance is uncertain. The specimen appears not to be digitized and we have not examined it.

Oenothera argillicola Mack., Shale Barren Evening-primrose, - S3/G3G4 (Hill 10689 MARY)

[Oenothera argillicola var. pubescens Core \& H. A. Davis]

Oenothera biennis L., Common Evening-primrose (Steele s.n. $\mathrm{PH})$

[Onagra biennis (L.) Scop.]

- Oenothera clelandii W. Dietr., P. H. Raven, \& W. L. Wagner, Cleland's Evening-primrose, † Waif (Coville 49 US)

Oenothera fruticosa L., Narrowleaf Sundrops (McAvoy 1099 DOV)

[Oenothera fruticosa ssp. microcarpa Fernald; O. fruticosa var. subglobosa (Small) Munz]

Oenothera grandis Smyth, Showy Evening-primrose, † (Shreve 1640 US)

[Oenothera laciniata Hill var. grandiflora (S. Watson) B. L. Rob.]

Oenothera bumifusa Nutt., Sea-beach Evening-primrose (Moldenke $13833 \mathrm{CM})$

Oenothera laciniata Hill, Cutleaf Evening-primrose (Killip 31895 US)

Oenothera nutans G. F. Atk. \& Bartlett, Nodding Evening-primrose (Steele 44 WVA)

[Oenothera biennis L. var. austromontana (Munz) Cronquist]

Oenothera oakesiana (A. Gray) J. W. Robbins ex S. Watson \& J. M. Coult., Oakes' Evening-Primrose (Tidestrom 5151 US)

Oenothera parviflora L., Northern Evening-primrose (Benner $6190 \mathrm{PH})$

Oenothera perennis L., Small Sundrops (Guttenberg 251 CM)

Oenothera tetragona Roth, Shrubby Sundrops (Cohen 27 MARY)

[Oenothera fruticosa ssp. glauca (Michx.) Straley; O. tetragona var. fraseri (Pursh) Munz]

Oenothera villosa Thunb. ssp. villosa, Hairy Evening-primrose (Munz $13475 \mathrm{NY}$ )

[Oenothera biennis L. var. canescens Torr. \& A. Gray]

\section{ORCHIDACEAE}

(Orchid Family)

Aplectrum byemale (Muhl. ex Willd.) Torr., Putty-root Orchid (McAvoy 4626 DOV)

[Aplectrum spicatum Britton, Sterns, \& Poggenb.]

Arethusa bulbosa (L.) Britton, Sterns, \& Poggenb., Dragon's Mouth, SX/G5 (Maxon \& Standley 389 US)

- Bletilla striata Rchb. f., Hyacinth Orchid, † Waif (Redman 6490 BALT)
Calopogon tuberosus (L.) Britton var. tuberosus, Tuberous Grasspink, S1/G5 (Killip \& Leonard 935 US)

[Calopogon pulchellus R. Br. ex Aiton; Limodorum tuberosum L.]

Cleistesiopsis divaricata (L.) Pansarin \& F. Barros, Rosebud Orchid, S1/G5 (Knapp Photo Voucher TAWES)

[Cleistes divaricata (L.) Ames; Pogonia divaricata (L.) R. Br.]

Corallorbiza maculata (Raf.) Raf., Summer Coralroot (Wherry s.n. US)

Corallorbiza odontorhiza (Willd.) Nutt., Fall Coralroot (Maxon 3839 US)

[Corallorhiza micrantha Chapm.]

Corallorbiza trifida Châtel., Yellow Coralroot, S1/G5. Reported from Garrett County but not collected due to rarity (Maryland Natural Heritage Program 2018). Also cited for Maryland by Magrath and Freudenstein (2002).

Corallorbiza wisteriana Conrad, Spring Coralroot, S1/G5 (Plitt s.n. US)

Cypripedium acaule Aiton, Pink Lady's Slipper (Leonard \& Leonard 6655 US)

Cypripedium candidum Muhl. ex Willd., White Lady's Slipper, - S1/G4 (Shriver et al. 569 CM)

Cypripedium parviflorum Salisb. var. parviflorum, Yellow Lady's Slipper, S3/G5 (Brown 1235 MARY)

[Cypripedium calceolus L. var. pubescens (Willd.) Correll; C. hirsutum Mill.; C. parviflorum var. pubescens (Willd.) O. W. C Knight; C. pubescens Willd.]

Cypripedium reginae Waters, Showy Lady's Slipper, SH/G4 (Redman 9918 BALT)

Dactylorhiza viridis (L.) R. M. Bateman, Pridgeon \& M. W. Chase var. virescens (Willd.) Baumbach, Longbract Frog Orchid, S1/G5 (Chrysler 208 MARY)

[Coeloglossum viride (L.) Hartman; Habenaria viridis (L.) R. Br. var. bracteata (Muhl. ex Willd.) A. Gray]

Epipactis helleborine (L.) Crantz, Helleborine, † (Knapp \& Frye 1406 TAWES)

Galearis spectabilis (L.) Raf., Showy Orchid (Ward s.n. US) [Galeorchis spectabilis (L.) Rydb.; Orchis spectabilis L.]

Goodyera pubescens (Willd.) R. Br., Downy Rattlesnake Plantain (Holler 837 US)

[Peramium pubescens (Willd.) MacMill.]

Goodyera repens (L.) R. Br., Lesser Rattlesnake Plantain, SH/ G5 (Stone s.n. PH)

Goodyera tesselata Lodd., Checkered Rattlesnake Plantain, SH/G5 (Fessenden s.n. US)

Hexalectris spicata (Walter) Barnhart, Spiked Crested Coralroot, S1/G5 (Fessenden \& Macurdy 5719a US) 
Isotria medeoloides (Pursh) Raf., Small Whorled Pagonia, SH/G2?, LT (Freeman \& Freeman s.n. US)

Isotria verticillata (Muhl. ex Willd.) Raf., Large Whorled Pagonia (Frye et al. s.n. TAWES)

Liparis liliifolia (L.) A. Rich. ex Lindl., Brown Widelip Orchid, S2S3/G5 (Boone s.n. TAWES)

Liparis loeselii (L.) Rich., Yellow Widelip Orchid, S1S2/G5 (Fessenden 5241 US)

Malaxis unifolia Michx., Green Adder's-mouth, S2/G5 (Maxon s.n. MARY)

Neottia bifolia (Raf.) Baumbach, Southern Twayblade, S3/G4 (Knapp 2377 DOV)

[Listera australis Lindl.]

Neottia cordata (L.) Rich., Heartleaf Twayblade, SH/G5 (Wherry s.n. PH)

[Listera cordata (L.) R. Br]

Neottia smallii (Wiegand) Szlach., Kidneyleaf Twayblade, S1/G4 (Gray s.n. NY)

[Listera smallii Wiegand]

Platanthera blephariglottis (Willd.) Lindl., White Fringed Orchid, S2/G4G5 (Sipple 1884 MARY)

[Habenaria blephariglottis (Willd.) Hook.]

Platanthera ciliaris (L.) Lindl., Yellow Fringed Orchid, S2/G5 (Chase s.n. US)

[Blephariglottis ciliaris (L.) Rydb.; Habenaria ciliaris (L.) R. Br. ex Aiton f.]

Platanthera clavellata (Michx.) Luer, Small Green Wood Orchid (Boone s.n. TAWES)

[Gymnadeniopsis clavellata (Michx.) Rydb.; Habenaria clavellata (Michx.) Spreng.]

Platanthera cristata (Michx.) Lindl., Crested Yellow Orchid, - S3/G5 (Killip 7249 US)

[Blephariglottis cristata (Michx.) Raf.; Habenaria cristata (Michx.) R. Br.]

Platanthera flava (L.) Lindl. var. flava, Southern Tubercled Rein Orchid, S2S3/G4T4 (Knapp \& McAvoy 3904 TAWES)

Platanthera flava (L.) Lindl. var. herbiola (R. Br.) Luer, Northern Tubercled Rein Orchid, S2S3/G4T4 (Ebert \& Holt s.n. TAWES)

[Habenaria flava (L.) R. Br. var. herbiola (R. Br.) Ames \& Correll]

Platanthera grandiflora (Bigelow) Lindl., Greater Purple Fringed Orchid, S2/G5 (Knapp Photo Voucher TAWES)

[Habenaria fimbriata (Aiton) R. Br.]

- Platanthera integra (Nutt.) A. Gray, Yellow Fringeless Orchid-see Excluded Flora section.

[Gymnadeniopsis integra (Nutt.) Rydb.; Habenaria integra (Nutt.) Spreng.]
Platanthera lacera (Michx.) G. Don, Green Fringed Orchid (Baltars 749 US)

[Blephariglottis lacera (Michx.) Farw.; Habenaria lacera (Michx.) R. Br.]

- Platanthera macrophylla (Goldie) P. M. Br., Greater Roundleaved Orchid-see Excluded Flora section.

- Platanthera nivea (Nutt.) Luer, Snowy Orchid—see Excluded Flora section.

Platanthera orbiculata (Pursh) Lindl., Round Leaved Orchid (Wherry s.n. PH)

Platanthera peramoena (A. Gray) A. Gray, Purple Fringeless Orchid, S1S2/G5 (Benedict 108 MARY)

[Blephariglottis peramoena (A. Gray) Rydb; Habenaria peramoena A. Gray; Platanthera fissa Lindl.]

Platanthera psycodes (L.) Lindl., Lesser Purple Fringed Orchid, - SH/G5 (Fessenden \& Rice 5307 US)

Platanthera shriveri P. M. Br., Shriver's Frilly Orchid, S1/G1 (Knapp \& Wiegand 2891 TAWES)

Pogonia ophioglossoides (L.) Ker Gawl., Rose Pogonia, S3/G5 (Frye 3140 TAWES)

Spiranthes arcisepala M. Pace, Appalachian Lady's Tresses (Longbottom et al. $27502 \mathrm{NY}$ )

Spiranthes cernua (L.) Rich., Nodding Lady's Tresses (Knapp 3200 DOV)

Spiranthes lacera (Raf.) Raf. var. gracilis (Bigelow) Luer, Slender Lady's Tresses (Canby s.n. DOV) [Spiranthes gracilis (Bigelow) Beck]

Spiranthes lacera (Raf.) Raf. var. lacera, Northern Slender Lady's Tresses (Pollard \& Catling s.n. US)

- Spiranthes laciniata Ames, Lacelip Lady's Tresses-see Excluded Flora section.

Spiranthes lucida (H. H. Eaton) Ames, Shining Lady's Tresses, - S1/G4 (Fessenden 5468 US)

Spiranthes ochrolenca (Rydb.) Rydb., Yellow Lady's Tresses, - S1/G4 (Boone s.n. TAWES)

Spiranthes odorata (Nutt.) Lindl., Marsh Lady's Tresses, SH/ G5 (Ditman s.n. MARY)

Spiranthes ovalis Lindl. var. erostellata Catling, October Lady's Tresses (Norton s.n. MARY)

Spiranthes praecox (Walter) S. Watson, Greenvein Lady's Tresses, SH/G5 (Hill \& Kratz $14446 a$ Assateague Island National Seashore Herbarium)

Spiranthes romanzoffiana Cham., Hooded Lady's Tresses (Taylor s.n. US)

Spiranthes tuberosa Raf., Little Lady's Tresses, S1?/G5 (Blake 8903 US)

[Spiranthes grayi Ames]

Spiranthes vernalis Engelm. \& A. Gray, Spring Lady's Tresses (Knapp 589 DOV) 
Tipularia discolor (Pursh) Nutt., Crippled Cranefly (Boone s.n. TAWES)

[Tipularia unifolia Britton, Sterns, \& Poggenb.]

Triphora trianthophora (Sw.) Rydb., Threebird's Orchid, S1/ G3G4 (Ulke s.n. US)

\section{OROBANCHACEAE}

(Broomrape Family)

Agalinis auriculata (Michx.) S. F. Blake, Earleaf False Foxglove, - S1/G3 (Henshaw s.n. US)

[Gerardia auriculata Michx.; Tomanthera auriculata (Michx.) Raf.]

Agalinis decemloba (Greene) Pennell, Sandplain Gerardia, - S1/G1, LE (Isanogle 7 US) [Agalinis acuta Pennell]

- Agalinis fasciculata (Elliott) Raf., Fascicled False Foxglovesee Excluded Flora section.

- Agalinis linifolia (Nutt.) Britton, Flaxleaf False Foxglove-see Excluded Flora section.

Agalinis maritima (Raf.) Raf. var. maritima, Saltmarsh False Foxglove (Leonard \& Leonard 15993 US)

[Gerardia maritima Raf.]

Agalinis obtusifolia Raf., Ten-lobe False Foxglove, SH/ G4G5Q (Leonard 5769 US) [Agalinis erecta (Walter) Pennell]

Agalinis purpurea (L.) Pennell, Large Purple False Foxglove (Naczi 8862 DOV)

[Gerardia purpurea L.]

Agalinis setacea (J. F. Gmel.) Raf., Threadleaf Gerardia, S2/G5? (Baltars 789 US)

[Gerardia setacea J. F. Gmel.]

Agalinis skinneriana (Alph. Wood) Britton, Pale False Foxglove, S1/G3G4 (McAvoy 4007 DOV)

Agalinis tenuifolia (Vahl) Raf., Slender False Foxglove (Leonard 19203 US)

[Gerardia tenuifolia Vahl]

Aphyllon uniflorum (L.) Torr. \& A. Gray, One-flowered Broomrape (Knapp $2728 \mathrm{DOV})$

[Orobanche uniflora L., Thalesia uniflora (L.) Britton]

Aureolaria flava (L.) Farw., Smooth Yellow False Foxglove, - S3/G5 (Randall Jr. 28 US) [Dasistoma flava (L.) Alph. Wood]

Aureolaria laevigata (Raf.) Raf., Appalachian Oak-leech, - SU/G5 (Reveal et al. 5000 MARY)

[Dasistoma laevigata (Raf.) Chapm.]

Aureolaria pedicularia (L.) Raf., Fernleaf Yellow False Foxglove (McAvoy $6721 \mathrm{DOV})$

Aureolaria virginica (L.) Pennell, Downy False Foxglove (McAvoy 2277 DOV)

[Aureolaria dispersa (Small) Pennell; A. microcarpa Pennell; Dasistoma virginica (L.) Britton]
Buchnera americana L., Bluehearts- SH/G5? (Blanchard s.n. $\mathrm{PH})$

Castilleja coccinea (L.) Spreng., Scarlet Indian-paintbrush, - S1/G5 (Smith s.n. BALT)

Conopholis americana (L.) Wallr., American Cancer-root (Beyersdorfer 73 US)

Epifagus virginiana (L.) Bartram, Beechdrops (Fosberg 56106 US) [Leptamnium virginianum (L.) Raf.]

Melampyrum lineare Desr., American Cow-wheat (Reveal 5537 MARY)

[Melampyrum lineare var. americanum (Michx.) Beauverd; M. lineare var. latifolium (Muhl. ex Britton) Beauverd; M. lineare var. pectinatum (Pennell) Fernald]

Orobanche minor Sm., Clover Broomrape, $\ddagger$ (Hyacinth 458 US)

- Orthocarpus bracteosus Benth., Rosy Owl's-clover, † Waif (Blake 11911 US)

Pedicularis canadensis L., Early Wood Lousewort (Redman 6659 BALT)

Pedicularis lanceolata Michx., Swamp Lousewort, S1/G5 (Knapp 2603 TAWES)

Schwalbea americana L., Chaffseed, SX/G2, LE (Commons s.n. PH)

[Schwalbea australis Pennell]

OXALIDACEAE

(Woodsorrel Family)

Oxalis colorea (Small) Eiten, Tufted Yellow Woodsorrel, † (Harms 9609 US)

Oxalis corniculata L., Creeping Woodsorrel, $†$ (Fosberg 23355 US)

Oxalis dillenii Jacq., Slender Yellow Woodsorrel (Allard 11854 US)

[Oxalis dillenii ssp. filipes (Small) G. Eiten; O. florida Salisb. var. filipes (Small) H. E. Ahles; O. stricta L.]

Oxalis florida Salisb., Slender Eastern Woodsorrel (Blake $10845 \mathrm{GH}$ )

Oxalis grandis Small, Great Yellow Woodsorrel (House 821 US)

Oxalis montana Raf., White Woodsorrel (Brighton Photo Voucher MPA)

Oxalis stricta L., Common Yellow Woodsorrel (Allard 11844 US)

[Oxalis cymosa Small; O. europaea Jordan; O. fontana Bunge]

Oxalis violacea L., Violet Woodsorrel (Hitchcock 12538 US) 


\section{PAPAVERACEAE}

(Poppy Family)

Adlumia fungosa (Aiton) Greene, Climbing Fumitory, S2/G4 (Boone s.n. TAWES)

- Argemone albiflora Hornem., Blue-stem Prickly-poppy, † Waif Reed (1964)

[Argemone alba F. Lestib.]

Argemone mexicana L., Mexican Prickly-poppy, † (Leonard 17568 US)

[Argemone leiocarpa Greene]

Capnoides sempervirens Borkh., Pale Corydalis, S3/G5 (Frye s.n. TAWES)

[Corydalis sempervirens (L.) Pers.]

Chelidonium majus L., Greater Celandine, † (Hotchkiss 5845 US)

- Corydalis aurea Willd., Golden Corydalis—see Excluded Flora section.

Corydalis flavula (Raf.) DC., Yellow Corydalis (Frye 766 TAWES)

[Capnoides flavulum (Raf.) Kuntze]

Corydalis incisa (Thunb.) Pers., Incised Fumewort, $†$ (Longbottom Photo Voucher MPA)

Dicentra canadensis (Goldie) Walpers, Squirrel-corn (Terrell 3938 US)

Dicentra cucullaria (L.) Bernh., Dutchman's Breeches (Hotchkiss 5814 US)

[Bicuculla cucullaria (L.) Millsp.]

Dicentra eximia (Ker Gawl.) Torr., Wild Bleedinghearts, S2/G4 (Eifrig s.n. CM)

[Bicuculla eximia (Ker Gawl.) Millsp.]

Fumaria officinalis L., Drug Fumitory, † (Ward s.n. US)

- Glaucium flavum Crantz, Yellow Hornpoppy, † Waif (Uhler et al. s.n. US)

- Hypecoum pendulum L., Nodding Hypecoum, † Waif Reed (1964)

Macleaya cordata (Willd.) R. Br., Plume Poppy, † (Longbottom \&. Thomas 5523 DOV)

Papaver argemone L., Pale Rough-fruited Poppy, † (Hemming s.n. MARY)

Papaver dubium L., Long-pod Poppy, † (Killip 31027 US)

- Papaver nudicaule L., Icelandic Poppy-see Excluded Flora section.

- Papaver orientale L., Oriental Poppy-see Excluded Flora section.

- Papaver rhoeas L., Corn Poppy, † Waif (Hill 16796 CLEMS)
- Papaver somniferum L., Opium Poppy-see Excluded Flora section.

Sanguinaria canadensis L., Bloodroot (McAvoy 3279 DOV)

Stylophorum diphyllum (Michx.) Nutt., Celandine Poppy, † (Miller 13 MARY)

PASSIFLORACEAE (Passionflower Family)

- Passiflora foetida L., Scarlet-fruited Passionflower, † Waif Reed (1964)

[Passiflora foetida var. isthmia Killip]

Passiflora incarnata L., Purple Passionflower, † (Knapp 568 DOV)

Passiflora lutea L., Yellow Passionflower (Steury 990918.8 US)

PAULOWNIACEAE (Paulownia Family)

Paulownia tomentosa (Thunb.) Steud., Royal Paulownia, $\ddagger$ (Edwin 334 US)

PENTHORACEAE (Ditch Stonecrop Family)

Penthorum sedoides L., Ditch Stonecrop (Killip 13335 US)

PHRYMACEAE (Lopseed Family)

Glossostigma cleistanthum W. R. Barker, Mudmats, † (Longbottom $12000 \mathrm{DOV})$

Mimulus alatus Aiton, Sharp-wing Monkeyflower (Fleming 2309 US)

Mimulus ringens L. var. ringens, Square-stem Monkeyflower (Fleming 2323 US)

Phryma leptostachya L., American Lopseed (Morton 2074 US)

PHYLLANTHACEAE (Leaf-flower Family)

Phyllanthus caroliniensis Walter ssp. caroliniensis, Carolina Leaf-flower, S3/G5 (Knapp 1685 DOV)

PHYTOLACCACEAE (Pokeweed Family)

Phytolacca americana L., Common Pokeweed (Beyersdorfer 90 US)

[Phytolacca decandra L.]

- Phytolacca bogotensis Kuntz, Southern Pokeweed, † Waif Reed (1964)

- Phytolacca icosandra L., Tropical Pokeweed, † Waif Reed (1964)

PLANTAGINACEAE (Plantain Family)

- Bacopa caroliniana (Walter) B. L. Rob., Carolina Water-hyssop-see Excluded Flora section.

[Hydrotrida caroliniana (Walter) Small] 
Bacopa innominata (M. Gómez) Alain, Tropical Water-hyssop, - SH/G3G5 (Tatnall 3961 DOV)

[Bacopa cyclophylla Fernald; B. stragula Fernald]

Bacopamonnieri (L.) Pennell, Monnier's Water-hyssop, SH/G5? (Canby s.n. NCU)

[Bramia monnieri (L.) Drake; Monniera monnieri (L.) Britton]

Bacopa rotundifolia Wettst., Disk Water-hyssop

[Herpestis rotundifolia C. F. Gaertn.; Macuillamia rotundifolia (Michx.) Raf.]

Pennell (1935) cited specimens from Maryland housed at PH; specimens have been on loan since 1983 and continue to be unavailable for study.

Callitriche heterophylla Pursh, Large Water-starwort (Davis $7235 \mathrm{BM})$

Callitriche palustris L., Vernal Water-starwort (Coville s.n. US)

Callitriche stagnalis Scop., Pond Water-starwort, † (Jensen s.n. US)

Callitriche terrestris Raf., Terrestrial Water-starwort (Baltars 302 MARY)

[Callitriche austinii Engelm.; C. deflexa A. Braun]

Chaenorhinum minus (L.) Lange ssp. minus, Dwarf Snapdragon, † (Baltars 2316 US)

Chelone glabra L., White Turtlehead (Hotchkiss s.n. US)

Chelone obliqua L., Red Turtlehead, S2/G4 (Allard 3716 US)

Cymbalaria muralis Gaertn., B. Meyer \& Scherber, Kenilworth Ivy, † (Arsène 605 US)

- Digitalis lanata Ehrh., Grecian Foxglove-see Excluded Flora section.

- Digitalis lutea L., Straw Foxglove-see Excluded Flora section.

Digitalis purpurea L., Purple Foxglove, † (Lutmerding Photo Voucher MPA)—perhaps a waif.

Gratiola aurea Pursh, Golden Hedge-hyssop (Knapp \& Frye 1112 DOV)

Gratiola neglecta Torr., Clammy Hedge-hyssop (Hitchcock 15834 US)

Gratiola ramosa Walter, Branched Hedge-hyssop, SH/G4G5 (Canby s.n. PH)

Gratiola virginiana L., Roundfruited Hedge-hyssop (Steury 050522.1 US)

[Gratiola sphaerocarpa Elliott; G. virginiana var. aestuariorum Pennell]

Gratiola viscidula Pennell, Short's Hedge-hyssop, S1/G4G5 (Knapp \& Harms 3320 TAWES)

Kickxia elatine (L.) Dumort., Sharp-point Toadflax, † (Leonard 619 US)

[Elatinoides elatine (L.) Wettst.]
Leucospora multifida (Michx.) Nutt., Narrowleaf Paleseed, $\dagger($ McAvoy 403 DOV)

Limosella australis R. Br., Mudwort, S2/G4G5 (Shull 306 US) [Limosella subulata Ives]

Linaria canadensis (L.) Dum. Cours., Common Toadflax (Allard 11192 US)

[Nuttallanthus canadensis (L.) D. A. Sutton]

Linaria vulgaris Mill., Butter and Eggs, † (Hitchcock 12680 US)

- Maurandya antirrhiniflora Humb. \& Bonpl. ex Willd., Snapdragon Vine, † Waif Reed (1964)

Mecardonia acuminata (Walter) Small var. acuminata, Purple Mecardonia, S2/G5 (Knapp \& Knapp 645 TAWES) [Monniera acuminata (Walter) Kuntze]

- Penstemon alluviorum Pennell, Lowland Beardtongue-see Excluded Flora section.

- Penstemon calycosus Small, Long-sepal Beardtongue-see Excluded Flora section.

Penstemon canescens Britton, Gray Beardtongue (Windler \& Stastny 3862 US)

Penstemon digitalis Nutt., Foxglove Beardtongue (Killip 12907 US)

Penstemon hirsutus (L.) Willd., Hairy Beardtongue (Fisher s.n. US)

Penstemon laevigatus Aiton, Smooth Beardtongue, SU/G5 (Benedict 5940 US) [Penstemon penstemon (L.) Britton]

Penstemon pallidus Small, Pale Beardtongue (Cusick 24298 CM) [Penstemon brevisepalus Pennell]

Plantago aristata Michx., Large-bract Plantain, † (Strong \& Simmons 3209 US)

Plantago cordata Lam., Heartleaf Plantain, SH/G4 (Sheldon s.n. US)

- Plantago heterophylla Nutt., Slender Plantain-see Excluded Flora section.

Plantago lanceolata L., English Plantain, $\ddagger$ (Bell et al. 163 US)

Plantago major L., Nipple-seed Plantain, † (Bell \& Ortiz 22 US)

- Plantago maritima L., Goose Tongue-see Excluded Flora section.

[Plantago maritima var. juncoides (Lam.) A. Gray]

Plantago pusilla Nutt., Dwarf Plantain, † (Norton 5062 MARY) [Plantago bybrida Bartram]

Plantago rugelii Decne., Black-seed Plantain (Hotchkiss 5459 US)

Plantago virginica L., Pale-seed Plantain (Blake s.n. US)

- Plantago wrightiana Decne., Wright's Plantain-see Excluded Flora section. 
Sophronanthe pilosa (Michx.) Small., Shaggy Hedge-hyssop (Knapp 919 DOV)

[Gratiola pilosa Michx; Tragiola pilosa (Michx.) Small \& Pennell]

Veronica agrestis L., Field Speedwell, † (Longbottom 18855 NY)

Veronica americana Schwein. ex Benth., American Speedwell (Standley 11773 US)

Veronica anagallis-aquatica L., Brook-pimpernel, † (Baltars 4086 US)

[Veronica glandifera Pennell]

Veronica arvensis L., Corn Speedwell, † (Allard 11086 US)

- Veronica austriaca L. ssp. teucrium (L.) D. A. Webb, Broadleaf Speedwell—see Excluded Flora section.

[Veronica latifolia L.]

- Veronica beccabunga L., European Speedwell-see Excluded Flora section.

Veronica chamaedrys L., Germander Speedwell, † (Longbottom 6054 DOV)

- Veronica cymbalaria Bodard, Glandular Speedwell-see Excluded Flora section.

Veronica filiformis Small, Slender Speedwell, † (Cusick \& Shelton 28941 CM)

Veronica hederifolia L., Ivyleaf Speedwell, † (Brummitt 20513 US)

- Veronica longifolia L., Longleaf Speedwell, † Waif (Stiteler s.n. PH)

[Pseudolysimachion longifolium Opiz]

Veronica officinalis L., Gypsy-weed, † (Fleming 2188 US)

Veronica peregrina L. ssp. peregrina, Neckweed (Longbottom 1588 DOV)

Veronica persica Poir., Bird's-eye Speedwell, † (Leonard 20559 US)

Veronica polita Fries, Wayside Speedwell, † (Allard 11089 US)

Veronica scutellata L., Marsh Speedwell, S1/G5 (Steele s.n. US)

Veronica serpyllifolia L. var. serpyllifolia, Thymeleaf Speedwell, $\dagger$ (Steele s.n. US)

Veronica sublobata M. A. Fisch., Pale Speedwell, † (Longbottom $27553 \mathrm{NY}$ )

Veronicastrum virginicum (L.) Farw., Culver's-root (Crawford s.n. US)

[Leptandra virginica (L.) Nutt.]

\section{PLATANACEAE}

(Plane Tree Family)

Platanus occidentalis L., Sycamore (Fisher s.n. US)

\section{PLUMBAGINACEAE}

(Leadwort Family)

Limonium carolinianum (Walter) Britton, Sea-lavender (Killip 32200 US)

[Limonium nashii Small]
POACEAE [Gramineae]

(Grass Family)

- Aegilops triuncialis L., Barbed Goatgrass, † Waif Reed (1964)

- Agrostis canina L., Velvet Bentgrass-see Excluded Flora section.

Agrostis capillaris L., Colonial Bentgrass, † (Norton s.n. MARY)

[Agrostis tenuis Sibth.; A. alba L. var. vulgaris (With.) Thurb.]

Agrostis elliottiana Schult., Elliott's Bentgrass, † (Hotchkiss 1952 US)

Agrostis gigantea Roth, Redtop, † (Smith 393 DOV) [Agrostis alba L.; A. stolonifera L. var. major Farw.]

Agrostis hyemalis (Walter) Britton, Sterns, \& Poggenb., Winter Bentgrass (McAvoy 6564 DOV)

- Agrostis mertensii Trin., Northern Bentgrass-see Excluded Flora section.

[Agrostis borealis Hartm.]

Agrostis perennans (Walter) Tuck., Upland Bentgrass (McAvoy 1962 DOV)

[Agrostis altissima (Walter) Tuck.; A. intermedia Schribn.]

Agrostis scabra Willd., Rough Bentgrass (Hermann 11553 US)

Agrostis stolonifera L., Creeping Bentgrass, † (McAvoy 4562 DOV)

[Agrostis alba L. var. stolonifera (L.) Scribn.; A. palustris Huds.; A. stolonifera var. compacta Hartm.]

Aira caryophyllea L., Silver Hairgrass, † (Longbottom 8826 DOV)

[Aspris caryophyllea (L.) Nash]

Aira elegans Willd. ex Gaudin, Annual Silver Hairgrass, † (Tatnall 4246 DOV)

[Aira caryophyllea L. var. capillaris (Host) Mutel; A. elegantissima Schur]

Aira praecox L., Early Silver Hairgrass, † (Canby s.n. DOV)

- Alloteropsis cimicina (L.) Stapf, Summergrass, † Waif Reed (1964)

[Coridochloa cimicina (L.) Nees ex Chase]

Alopecurus aequalis Sobol., Shortawn Foxtail, † (Longbottom 7156 DOV)

Alopecurus carolinianus Walter, Carolina Foxtail (Knapp 1873 DOV)

[Alopecurus ramosus Poir.]

Alopecurus geniculatus L., Water Foxtail, † (Ward s.n. US)

Alopecurus myosuroides Huds., Slender Meadow Foxtail, † (Chase 6899 US)

Alopecurus pratensis L., Meadow Foxtail, † (Stewart s.n. US)

Amphicarpum amphicarpon (Pursh) Nash, Pursh's Blue Maidencane, S3/G4 (Naczi 11551 DOV)

[Amphicarpum purshii Kuntze] 
Andropogon gerardi Vitman, Big Bluestem (Ebert o Holt 142EH DOV)

[Andropogon gerardii-orthographic variant; A. furcatus Muhl. ex Willd.; A. provincialis Lam.]

- Andropogon glaucopsis Elliott, Purple Bluestem—see Excluded Flora section.

[Andropogon glomeratus (Walter) Britton, Sterns, \& Poggenb. var. glaucopis (Elliott) C. Mohr]

Andropogon glomeratus (Walter) Britton, Sterns, \& Poggenb. var. glomeratus, Bushy Bluestem (Longbottom 14306 MARY)

[Andropogon virginicus L. var. abbreviatus (Hack.) Fernald \& Griscom]

Andropogon glomeratus (Walter) Britton, Sterns, \& Poggenb. var. hirsutior (Hack.) C. Mohr, Hairy Bluestem (Hermann 9973 NY)

[Andropogon virginicus L. var. hirsutior (Hack.) Hitchc.]

Andropogon gyrans Ashe, Elliott's Bluestem (Tatnall 4411 DOV)

[Andropogon elliottii var. gracilior Hack.; A. elliottii var. projectus Fernald \& Griscom]

Andropogon ternarius Michx., Splitbeard Bluestem (McAvoy 1961 DOV)

[Andropogon argyraeus Schult.; A. elliottii Chapm]

Andropogon virginicus L. var. virginicus, Broomsedge (McAvoy 3156 DOV)

[Andropogon tetrastachyus Elliott]

Anthoxanthum hirtum (Schrank) Y. Schouten \& Veldkamp, Holy Grass, S1/G4G5 (Reveal \& Broome 5133 MARY) [Hierochloe hirta (Schrank) Borbás; H. odorata (L.) P. Beauv.]

Anthoxanthum odoratum L., Sweet Vernal Grass, † (McAvoy 977 DOV)

- Apera spica-venti (L.) P. Beauv., Loose Silkybent, † Waif (Norton s.n. MARY)

[Agrostis spica-venti L.]

- Apluda mutica L., Mauritian Grass, † Waif (Reed 1964)

- Aristida adscensionis L., Sixweeks Threeawn, † Waif (Reed 32826 US)

Aristida dichotoma Michx. var. curtissii A. Gray, Curtis's Threeawn (McAvoy 2026 DOV)

[Aristida curtissii (A. Gray) Nash]

Aristida dichotoma Michx. var. dichotoma, Churchmouse Threeawn (McAvoy 2026 DOV)

Aristida lanosa Muhl. ex Elliott, Woolysheath Threeawn, S1/ G5 (McAvoy 753 DOV)

[Aristida lanata Poir.]

Aristida longespica Poir. var. geniculata (Raf.) Fernald, Northern Slim-spike Threeawn (Otis s.n. DOV)

[Aristida intermedia Scribn. \& Ball]
Aristida longespica Poir. var. longespica, Southern Slim-spike Threeawn (Knapp 1043 TAWES)

[Aristida gracilis Elliott]

Aristida oligantha Michx., Prairie Threeawn (Knapp 1127 TAWES)

Aristida purpurascens Poir., Arrowfeather Threeawn (McAvoy $6722 \mathrm{DOV})$

Aristida tuberculosa Nutt., Seaside Threeawn, S3/G5 (Hamilton 190 TAWES)

Aristida virgata Trin., Wand-like Threeawn, S1S2/G5 (Knapp 1076 TAWES)

[Aristida purpurascens Poir. var. virgata (Trin.) Allred]

Arrhenatherum elatius (L.) P. Beauv. ex J. Presl \& C. Presl var. bulbosum Spenn., Bulbous Oatgrass, † (Norton s.n. MARY)

Arrhenatherum elatius (L.) P. Beauv. ex J. Presl \& C. Presl var. elatius, Tall Oatgrass, $†$ (Redden 002 DOV)

Arthraxon bispidus (Thunb.) Makino, Small Carpetgrass, $\ddagger$ (Redman s.n. TAWES)

[Arthraxon ciliaris P. Beauv. var. cryptatherus Hack.; A. hispidus var. cryptatherus (Hack.) Honda]

- Arundinaria gigantea (Walter) Muhl., Giant Cane-see Excluded Flora section.

Arundinaria tecta (Walter) Muhl., Switchcane, S2/G5 (McAvoy 2695 DOV) [Arundinaria gigantea (Walter) Muhl. ssp. tecta (Walter) McClure]

Arundo donax L., Giant Reed, $\ddagger$ (Otis s.n. DOV)

Avena fatua L., Wild Oat, † (Baltars 634 US)

Avena sativa L., Common Oat, † (Painter 448 US)

Avenella flexuosa (L.) Dejer., Wavy Hairgrass (McAvoy 2713 DOV)

[Aira flexuosa L.; Deschampsia flexuosa (L.) Trin.]

Axonopus furcatus (Flüggé) A. Hitchcock, Big Carpetgrass, S2?/G5 (McAvoy 6087 DOV)

- Bothriochloa pertusa (L.) A. Camus, Pitted Beardgrass, † Waif Reed (1964)

[Andropogon pertusus (L.) Willd.]

- Bouteloua aristidoides (Kunth) Griseb., Needle Grama, † Waif (Reed 32727 US)

- Bouteloua barbata Lag., Sixweeks Grama, † Waif (Reed 32824 US)

Bouteloua curtipendula (Michx.) Torr., Sideoat Grama, S2/ G5 (Boone 840914 TAWES)

- Bouteloua hirsuta Lag., Hairy Grama-see Excluded Flora section. 
Brachyelytrum aristosum (Michx.) P. Beauv. ex Trel., Northern Long-awned Wood Grass (Boone s.n. TAWES)

[Brachyelytrum erectum (Schreb.) P. Beauv var. septentrionale Babel; B. septentrionale (Babel) G. C. Tucker]

Brachyelytrum erectum (Schreb.) P. Beauv, Long-awned Wood Grass (Boone s.n. TAWES)

- Briza media L., Perennial Quaking Grass—see Excluded Flora section.

Briza minor L., Little Quaking Grass, $\ddagger$ (Knapp 1878 DOV)

Bromus arvensis L., Field Brome, † (Ditman s.n. MARY)

- Bromus briziformis Fisch. \& C. A. Mey, Rattlesnake Brome, $\dagger$ Waif (Norton s.n. US)

Bromus catharticus Vahl, Rescue Grass, † (Norton s.n. MARY) [Bromus unioloides Kunth]

Bromus ciliatus L., Fringed Brome, SH/G5 (Braun s.n. US) [Bromus dudleyi Fernald]

Bromus commutatus Schrad., Meadow Brome, † (Frye 3133 TAWES)

- Bromus diandrus Roth, Ripgut Brome, † Waif (Chase 6907 US) [Bromus rigidus Roth]

Bromus hordeaceus L., Soft Brome, † (Frye 803 TAWES)

[Bromus mollis L.]

Bromus inermis Leyss., Smooth Brome, † (Frye 631 TAWES)

Bromus japonicus Thunb., Field Brome, † (Frye 583 TAWES)

Bromus kalmii A. Gray, Arctic Brome, SH/G5 (Steele 95 US)

Bromus latiglumis (Shear) Hitchc., Early-leaf Brome, S1/G5 (Thompson s.n. TAWES)

[Bromus altissimus Pursh; B. incanus (Schear) Hitchc.; B. purgans var. incanus Shear]

- Bromus madritensis L., Compact Brome-see Excluded Flora section.

Bromus nottowayanus Fernald, Satin Brome, S3S4/G5 (Naczi 9323 DOV)

Bromus pubescens Muhl. ex Willd., Hairy Woodland Brome (Naczi 9322 DOV)

[Bromus laeviglumis (Scribn.) Hitchc.; B. purgans auct. non L.]

Bromus racemosus L., Bald Brome, † (Knapp 1874 DOV)

- Bromus rubens L., Red Brome-see Excluded Flora section.

Bromus secalinus L., Rye Brome, † (Tidestrom 12074 DOV)

Bromus sterilis L., Poverty Brome, † (Otis s.n. DOV)

Bromus tectorum L., Cheatgrass, $\uparrow$ (Tucker s.n. DOV)

Calamagrostis arenaria (L.) Roth, European Beachgrass, $\dagger$ (Hitchcock 7889 US)

[Ammophila arenaria (L.) Link]

Calamagrostis breviligulata (Fernald) Saarela ssp. breviligulata, American Beachgrass

(Cooley s.n. TAWES)

[Ammophila breviligulata Fernald]
Calamagrostis canadensis (Michx.) P. Beauv. var. canadensis, Bluejoint (Boone s.n. TAWES)

Calamagrostis porteri A. Gray, Porter's Reedgrass, S3/G5 (Frye et al. 6002 TAWES)

- Cenchrus echinatus L., Southern Sandbur, † Waif Reed (1964)

Cenchrus longispinus (Hack.) Fernald, Mat Sandbur (Baltars 3215 US)

[Cenchrus carolinianus Walter]

Cenchrus purpurascens Thunb., Chinese Fountaingrass, $\ddagger$ (Longbottom 12372 US)

[Cenchrus compressus (R. Br.) Morrone; Pennisetum alopecuroides (L.) Spreng.]

Cenchrus spicatus (L.) Cav., Pearl Millet, † (Baltars 3198 US) [Cenchrus americanus (L.) Morrone; Pennisetum glaucum (L.) R. Br.]

- Cenchrus spinifex Cav., Coastal Sandbur-see Excluded Flora section.

[Cenchrus incertus M. A. Curtis; C. pauciflorus Benth.]

Cenchrus tribuloides L., Sand Dune Sandbur (Longbottom 3338 US)

[Cenchrus macrocephalus Scribn.]

Chasmanthium latifolium (Michx.) Yates, Indian Woodoats (Hirst \& Wilson 623 DOV)

[Uniola latifolia Michx.]

Chasmanthium laxum (L.) Yates, Slender Woodoats (Knapp 2805 DOV)

[Uniola laxa (L.) Britton, Sterns, \& Poggenb.]

Chloris verticillata Nutt., Tumble Windmill Grass, † (Goldberg s.n. US)

Chloris virgata Sw., Feather Fingergrass, † (Cusick 37587 CM)

Cinna arundinacea L., Sweet Woodreed (Longbottom 6839 DOV)

Cinna latifolia Griseb., Drooping Woodreed, S3/G5 (Reveal et al. 5023 MARY)

Coleataenia anceps (Michx.) Soreng ssp. anceps, Beaked Panic Grass (Terrell 3294 US)

[Panicum anceps Michx. var. anceps]

- Coleataenia anceps (Michx.) Soreng ssp. rhizomata (Hitchc. \& Chase) Soreng, Small Beaked Panic Grass-see Excluded Flora section.

[Panicum anceps Michx. ssp. rhizomatum (Hitchc. \& Chase) Freckmann \& Lelong]

Coleataenia longifolia (Torr.) Soreng ssp. combsii (Scribn. \& Ball) Soreng, Comb's Panic Grass (Novik 421 US)

[Panicum longifolium Torr. var. combsii (Scribn. \& Ball) Fernald; P. rigidulum Bosc ex Nees var. combsii (Schribn. \& Ball) Lelong] 
Coleataenia longifolia (Torr.) Soreng ssp. longifolia, Longleaved Panic Grass (Sarver s.n. TAWES)

[Panicum longifolium Torr. var. longifolium; P. rigidulum Bosc ex Nees; P. rigidulum ssp. pubescens (Vasey) Freckmann \& Lelong]

Coleataenia rigidula (Bosc ex Nees) LeBlond ssp. condensa (Nash) LeBlond, Dense Panic Grass (Knapp 1074E NCU)

[Panicum rigidulum Bosc ex Nees var. condensum (Nash) F. Seym.]

Coleataenia rigidula (Bosc ex Nees) LeBlond ssp. rigidula, Redtop Panic Grass (Whitcomb et al. s.n. MARY)

[Panicum agrostoides Spreng.; P. condensum Nash; P. elongatum Pursh var. ramosius C. Mohr; P. rigidulum Bosc ex Nees var. rigidulum]

Coleataenia stipitata (Nash) LeBlond, Tall Flat Panic Grass (Earle 2308 US)

[Coleataenia longifolia (Torr.) Soreng ssp. elongata (Pursh) Soreng; Panicum rigidulum Bosc ex Nees ssp. elongatum (Pursh) Freckmann \& Lelong]

Cynodon dactylon (L.) Pers., Bermuda Grass, $\ddagger$ (Longbottom 5299 DOV)

[Capriola dactylon (L.) Kuntze]

- Cynosurus cristatus L., Crested Dog's-tail Grass, † Waif (Novik s.n. MARY)

- Cynosurus echinatus L., Bristly Dog's-tail Grass, † Waif (Baltars 3125 US)

Dactylis glomerata L., Orchard Grass, † (Longbottom 13307 DOV)

Dactyloctenium aegyptium (L.) Willd., Egyptian Grass, † (Pond s.n. MARY)

- Dactyloctenium germinatum Hack., Sudan Crowfoot Grass, † Waif Reed (1964)

Danthonia compressa Austin, Flattened Oatgrass (Chase 7557 US)

Danthonia sericea Nutt., Downy Danthonia (Hirst 688 DOV)

Danthonia spicata (L.) P. Beauv. ex Roem. \& Schult., Poverty Oat Grass (McAvoy 1059 DOV)

- Dasyochloa pulchella (Kunth) Willd. ex Rydb., Low Wollygrass, † Waif (Reed 32827 US)

[Tridens pulchellus (Kunth) Hitchc.]

Deschampsia caespitosa (L.) P. Beauv. ssp. glauca (C. Hartm.) C. Hartm., Tufted Hairgrass, S1/G5 (Fessenden 5647 US) [Aira caespitosa L.]

- Diarrhena americana P. Beauv., American Beak Grass-see Excluded Flora section.

[Diarina festucoides Raf.]
Diarrhena obovata (Gleason) Brandenburg, Beak Grass, † (Frye \& Peterson 6047 TAWES)

Dichanthelium aciculare (Desv. ex Poir.) Gould \& C. A. Clark, Needle-leaf Rosettegrass, S2?/G4G5 (Knapp 108-03 NCU) [Panicum aciculare Desv. ex Poir.; P. ovinum Scribn. \& J. G. Sm.]

Dichanthelium aculeatum (Hitchc. \& Chase) LeBlond, Prickly Rosettegrass (Chase $2520 \mathrm{NY}$ )

Dichanthelium acuminatum (Sw.) Gould \& C. A. Clark, Woolly Rosettegrass (Naczi et al. $15526 \mathrm{NY}$ )

[Dichanthelium acuminatum var. thurowii (Scribn. \& J. G. Sm.) Gould \& C. A. Clark; Panicum acuminatum Sw. var. acuminatum; P. acuminatum var. thurowii (Scribn. \& J. G. Sm.) C. F. Reed; P. auburne Ashe; P. thurowii Scribn. \& J. G. Sm.]

Dichanthelium angustifolium (Elliott) Gould, Narrow-leaved Rosettegrass (Canby s.n. US)

[Panicum angustifolium Elliott]

Geographic location of specimen is uncertain, with label stating, "Eastern Shore of Maryland and Virginia."

Dichanthelium annulum (Ashe) LeBlond, Ringed Rosettegrass, S1/G5 (Chase s.n. PH)

- Dichanthelium arenicoloides (Ashe) LeBlond, Sandy Woods Rosettegrass-see Excluded Flora section.

[Panicum arenicoloides Ashe]

Dichanthelium bicknellii (Nash) LeBlond, Bicknell's Rosettegrass SU/G5 (Chase $3783 \mathrm{NY)}$

[Panicum bicknellii Nash]

- Dichanthelium boreale (Nash) Freckmann, Northern Rosettegrass-see Excluded Flora section.

[Panium boreale Nash]

Dichanthelium boscii (Poir.) Gould \& C. A. Clark, Bosc's Rosettegrass (Knapp 1214 DOV)

[Panicum boscii Poir.]

Dichanthelium clandestinum (L.) Gould, Deer-tongue Rosettegrass (Tatnall 2975 DOV)

[Panicum clandestinum L.]

Dichanthelium columbianum (Scribn.) Freckmann, American Rosettegrass (Chase s.n. PH)

[Dichanthelium acuminatum (Sw.) Gould \& C. A. Clark ssp. columbianum (Scribn.) Freckmann \& Lelong; Panicum acuminatum Sw. var. columbianum (Scribn.) Lelong; P. columbianum Scribn.; P. tsugetorum Nash]

Dichanthelium commonsianum (Ashe) Freckmann, Commons' Rosettegrass (McAvoy 6390 DOV)

Dichanthelium commutatum (Schult.) Gould ssp. commutatum, Variable Rosettegrass (McAvoy 6553 DOV)

[Dichanthelium commutatum ssp. joorii (Vasey) Freckmann \& Lelong; Panicum commutatum Schult; P. commutatum var. joorii (Vasey) Fernald; P. equilaterale Scribn.; P. joorii Vasey; P. mutabile Scribn. \& J. G. Sm.]

Dichanthelium commutatum (Schult.) Gould ssp. ashei (Ashe) Freckmann \& Lelong, Ashe’s Rosettegrass (McAvoy 2298 DOV) 
Dichanthelium depauperatum (Muhl.) Gould, Starved Rosettegrass (Frye 5093 \& Knapp TAWES)

[Panicum depauperatum Muhl.]

- Dichanthelium dichotomum (L.) Gould ssp. roanokense (Ashe) Freckmann \& Lelong, Roanoke Rosettegrass-see Excluded Flora section.

[Panicum dichotomum L. var. roanokense (Ashe) Lelong; P. roanokense Ashe]

Dichanthelium dichotomum (L.) Gould var. dichotomum, Forked Rosettegrass (Knapp 2181 NCU)

[Panicum barbulatum Michx.; P. dichotomum L. var. dichotomum; P. sphagnicola Nash]

Dichanthelium dichotomum (L.) Gould var. nitidum (Lam.) LeBlond, Shining Rosettegrass (Knapp 925 NCU)

Dichanthelium ensifolium (Baldwin ex Elliott) Gould, Smallleaved Rosettegrass (Hirst \& Wilson 401 DOV)

[Dichanthelium dichotomum (L.) Gould var. ensifolium (Baldwin ex Elliott) Gould \& Clark; Panicum ensifolium Baldwin ex Elliott]

Dichanthelium filiramum (Ashe) LeBlond, Hairy Needle-leaved Rosettegrass (Frye 6170 TAWES)

[Panicum chrysopsidifolium Nash]

Dichanthelium lanuginosum (Elliot) Gould, Wooly Rosettegrass (McAvoy 1570 DOV)

[Dichanthelium acuminatum (Sw.) Gould \& C. A. Clark var. faciculatum (Torr.) Freckmann; Panicum acuminatum var. fasciculatum (Torr.) Freckmann; P. fasciculatum (Torr.) Freckmann \& Lelong; P. huachucae Ashe; P. lanuginosum Elliott; P. tennesseense Ashe]

Dichanthelium latifolium (Linnaeus) Gould \& C. A. Clark, Broad-leaved Witchgrass (Frye 5551 TAWES)

[Panicum latifolium Linnaeus]

Dichanthelium laxiflorum (Lam.) Gould, Open-flowered Rosettegrass, S1?/G5 (Chase 2316 NY)

[Panicum laxiflorum Lam.; P. pyriforme Nash; P. xalapense Kunth]

Dichanthelium leucothrix (Nash) Freckman, Roughish Rosettegrass, SU/G4?Q (Knapp 978 TAWES)

[Dichanthelium acuminatum (Sw.) Gould \& C. A. Clark ssp. leucothrix (Nash) Freckmann \& Lelong; Panicum acuminatum Sw. var. leucothrix (Nash) Lelong; P. leucothrix Nash; P. parvispiculum Nash]

Dichanthelium lindheimeri (Nash) Gould, Lindheimer's Rosettegrass (McAvoy 4204 DOV)

[Dichanthelium acuminatum (Sw.) Gould \& C. A. Clark ssp. lindheimeri (Nash) Freckmann \& Lelong; D. acuminatum var. lindheimeri (Nash) Gould \& C. A. Clark; Panicum acuminatum Sw. var. lindheimeri (Nash) Lelong; P. lindbeimeri $\mathrm{Nash}]$

Dichanthelium linearifolium (Scribn.) Gould, White-haired Rosettegrass (Naczi et al. $15451 \mathrm{NY}$ )

[Panicum linearifolium Scribn.; P. werneri Scribn.]

Dichanthelium longiligulatum (Nash) Freckman, Long-ligule Rosettegrass (Knapp 978 TAWES)
[Dichanthelium acuminatum (Sw.) Gould \& C. A. Clark ssp. longiligulatum (Nash) Freckmann \& Lelong; Panicum acuminatum Sw. var. longiligulatum (Nash) Lelong; P. longiligulatum $\mathrm{Nash}]$

Dichanthelium lucidum (Ashe) LeBlond, Bog Rosettegrass (Sipple $1877 \mathrm{PH}$ )

[Dichanthelium dichotomum (L.) Gould ssp. lucidum (Ashe) Freckmann \& Lelong; Panicum dichotomum L. var. lucidum (Ashe) Lelong; P. lucidum Ashe]

Dichanthelium mattamuskeetense (Ashe) Mohlenbr., Matamuskeet Rosettegrass (McAvoy 5187 DOV)

[Panium mattamuskeetense Ashe]

Dichanthelium meridionale (Ashe) Freckman, Matting Rosettegrass (Hirst 326 DOV)

[Dichanthelium acuminatum (Sw.) Gould \& C. A. Clark ssp. implicatum (Scribn.) Freckmann \& Lelong; Panicum acuminatum Sw. var. implicatum (Scribn.) Beetle; P. acuminatum var. unciphyllum (Trin.) Lelong; P. implicatum Scribn.; P. meridionale Ashe]

Dichanthelium microcarpon (Ell.) Mohlenbr., Small-fruited Rosettegrass (Naczi et al. 15478 NY)

[Dichanthelium dichotomum (L.) Gould var. ramulosum (Torr.) LeBlond]

Dichanthelium oligosanthes (Schult.) Gould var. oligosanthes, Fewflowered Rosettegrass, S2S3?/G5T5? (Frye 5947 TAWES) [Panicum oligosanthes Schult.]

Dichanthelium oligosanthes (Schult.) Gould var. scribnerianum (Nash) Gould, Scribner's Rosettegrass, S2/G5T5? (Naczi et al. $15463 \mathrm{NY)}$

- Dichanthelium ovale (Elliott) Gould \& C. A. Clark, Low Stiff Rosettegrass-see Excluded Flora section.

[Panicum ovale Elliott]

Dichanthelium polyanthes (Schult.) Mohlenbr., Round-fruited Rosettegrass (Tatnall 374 DOV)

[Panicum polyanthes Schult.]

Dichanthelium ravenelii (Scribn. \& Merr.) Gould, Ravenel's Rosettegrass, SH/G5 (Chase $3796 \mathrm{PH}$ )

[Panicum ravenelii Scribn. \& Merr.]

Dichanthelium scabriusculum (Elliott) Gould \& C. A. Clark, Tall Swamp Rosettegrass, S1/G4 (McAvoy 319 DOV)

[Panicum scabriusculum Elliott]

Dichanthelium scoparium (Lam.) Gould, Velvet Rosettegrass (Tatnall 381 DOV)

[Panicum pubescens Lam.; P. scoparium Lam.; P. viscidum Elliott]

Dichanthelium sphaerocarpon (Elliott) Gould, Sphere-fruited Rosettegrass (McAvoy 6388 DOV)

[Panicum sphaerocarpon Elliott]

Dichanthelium spretum (Schult.) Freckman, Eaton's Rosettegrass (Naczi $15531 \mathrm{NY)}$

[Dichanthelium acuminatum (Sw.) Gould \& C. A. Clark ssp. spretum (Schult.) Freckmann \& Lelong; Panicum acuminatum Sw. var. densiflorum (E. L. Rand \& Redfield) Lelong; P. paucipilum Nash; P. spretum Schult.] 
Dichanthelium tenue (Muhl.) Freckmann \& LeLong, Whiteedged Rosettegrass (Naczi et al. 14816 NY)

[Panicum tenue Muhl.]

Dichanthelium villosissimum (Nash) Freckmann, White-haired Rosettegrass (McAvoy 1659 DOV)

[Dichanthelium ovale (Elliott) Gould \& C. A. Clark ssp. villosissimum (Nash) Freckmann \& Lelong; Panicum haemacarpon Ashe; P. ovale Elliott var. villosum (A. Gray) Lelong; P. villosissimum Nash; P. xanthospermum Scribn. \& Mohr]

Dichanthelium wrightianum (Scribn.) Freckmann, Wright's Rosettegrass, S1/G4 (Hirst \& Wilson 556 DOV) [Panicum wrightianum Scribn.]

Dichanthelium yadkinense (Ashe) Mohlenbr., Spotted-sheath Rosettegrass (Chase $3772 \mathrm{PH}$ )

[Dichanthelium dichotomum (L.) Gould ssp. yadkinense (Ashe) Freckmann \& Lelong; Panicum dichotomum L. var. yadkinense (Ashe) Lelong; P. yadkinense Ashe]

Digitaria ciliaris (Retz.) Koeler, Southern Crab Grass (Reed 65409 US)

[Digitaria adscendens (Kunth) Henr.; D. sanguinalis (L.) Scop. var. ciliaris (Retz.) Parl.]

Digitaria cognata (Schult.) Pilg., Fall Crab Grass (Smith s.n. US) [Leptoloma cognata (Schult.) Chase]

Digitaria filiformis (L.) Koeler, Slender Crab Grass (Leonard $6117 a$ US)

Digitaria ischaemum (Schreb.) Schreb. ex Muhl., Smooth Crab Grass, † (Hitchcock 15713 US)

[Digitaria ischaemum var. mississippiensis Fernald]

- Digitaria longiflora (Retz.) Pers., Indian Crab Grass, † Waif Reed (1964)

Digitaria sanguinalis (L.) Scop., Northern Crab Grass, † (Grant 89-00495 DOV)

Digitaria villosa (Walter) Pers., Shaggy Crab Grass, S1?/G5 (McAvoy 7142 DOV)

- Dinebra retroflexa (Vahl) Panze, Viper Grass, † Waif Reed (1964)

- Diplachne dubia (Kunth) Scribn., Green Sprangletop, † Waif Reed (1964)

[Leptochloa dubia (Kunth) Nees]

Diplachne fusca (Lam.) P. Beauv. ex Roem. \& Schult., Malabar Sprangletop, † (Leonard 626 US)

[Diplachne maritima E. P. Bicknell; Leptochloa fascicularis (Lam.) A. Gray; L. maritima (E. P. Bicknell) LeBlond \& Sorrie]

Distichlis spicata (L.) Greene, Salt Grass (Longbottom 6911 DOV)

Echinochloa colona (L.) Link, Jungle Rice, $¥$ (Longbottom 20026 NY)

[Panicum colonum L.]
Echinochloa crus-galli (L.) P. Beauv., Barnyard Grass, $\ddagger$ (Seyfried \& Outten CEP86-814 DOV)

[Panicum crus-galli L.]

- Echinochloa crus-pavonis (Kunth) Schult., Gulf Cockspur Grass, † Waif Reed (1964)

[Echinochloa crus-galli (L.) P. Beauv. var. zelayensis (Kunth) Hitchc.]

Echinochloa frumentacea Link, Billion-Dollar Barnyard Grass, † (Otis s.n. DOV)

[Echinochloa crus-galli (L.) P. Beauv. var. frumentacea (Link) Wright]

Echinochloa muricata (P. Beauv.) Fernald var. microstachya Wiegel, Small-spiked Barnyard Grass (Hall s.n. MARY)

[Echinochloa crus-galli (L.) P. Beauv. var. mitis (Pursh) Petermann; E. microstachya (Wiegel) Rydb.]

Echinochloa muricata (P. Beauv.) Fernald var. muricata, Rough Barnyard Grass (McAvoy 5659 DOV)

Echinochloa walteri (Pursh) Heller, Walter's Barnyard Grass (Walker 3941 US)

[Panicum walteri Pursh]

Eleusine indica (L.) Gaertn., Goose Grass, $\ddagger$ (Longbottom 5295 DOV)

Elymus canadensis L., Canada Wild Rye (Longbottom et al. 6261 DOV)

Elymus glabriflorus (Vasey) Scribn. \& Ball var. australis (Scribn. \& Ball) J. J. N. Campb., Southeastern Wild Rye (Gladu s.n. MARY)

Elymus glabriflorus (Vasey) Scribn. \& Ball var. glabriflorus, Smooth Southeastern Wild Rye (Tatnall 383 DOV)

Elymus hystrix L., Bottlebrush Grass (Knapp 2834 DOV) [Hystrix patula Moench; Elymus hystrix var. bigelovianus (Fernald) Bowden]

Elymus macgregorii R. Brooks \& J. J. N. Campb., Early Wildrye (Otis s.n. DOV)

Elymus repens (L.) Gould, Quack Grass (Baltars 3142 US) [Agropyron repens (L.) P. Beauv.; Elytrigia repens (L.) Desv. ex Nevski]

Elymus riparius Wiegand, Riverbank Wildrye (Knapp 2815 DOV)

- Elymus trachycaulus (Link) Gould ssp. glaucus (Pease \& A. H. Moore) Cody, Bearded Wheatgrass-see Excluded Flora section.

[Agropyron trachycaulum (Link) Malte ex H. F. Lewis var. glaucum (Pease \& A. H. Moore) Malte]

- Elymus trachycaulus (Link) Gould ssp. subsecundus (Link) Á. Löve \& D. Löve, Slender Wheatgrass-see Excluded Flora section.

[Agropyron subsecundum (Link) Hitchc.; A. trachycaulum (Link) Malte ex H. F. Lewis var. unilaterale (Cassidy) Malte]

Elymus villosus Muhl. ex Willd., Silky Wildrye (McAvoy 6633 DOV)

[Elymus striatus Willd. var. villosus (Muhl. ex Willd.) A. Gray; E. villosus var. arkansanus (Scribn. \& C. R. Ball) J. J. N. Campb.] 
Elymus virginicus L. var. halophilus (E. P. Bicknell) Wiegand, Salt-marsh Wildrye (Otis s.n. DOV)

[Elymus halophilus E. P. Bicknell]

Elymus virginicus L. var. intermedius (Vasey ex S. Watson \& J. M. Coult.) Bush, Intermediate Wildrye (Norton s.n. MARY) [Elymus virginicus var. hirsutiglumis (Scribn.) Hitchc.]

Elymus virginicus L. var. virginicus, Virginia Wildrye (Knapp 2809 DOV)

[Elymus striatus Willd.]

- Enneapogon desvauxii Desv. ex P. Beauv., Nineawn Pappusgrass, † Waif Reed (1964)

- Eragrostis barrelieri Daveau, Mediterranean Lovegrass, † Waif (Reed 43644 US)

Eragrostis capillaris (L.) Nees, Lace Lovegrass (Leonard 21046 US)

Eragrostis cilianensis (All.) Vignolo ex Janch., Stinking Lovegrass, † (Killip \& Swallen 31905 US)

[Eragrostis major Host]

Eragrostis curvula (Schrad.) Nees, Weeping Lovegrass, $\dagger$ (Ables23701 US)

- Eragrostis cylindriflora Hochst., Cylinderflowered Lovegrass, † Waif Reed (1964)

[Eragrostis horizontalis Peter]

Eragrostis frankii (Fisch., C. A. Mey. \& Avé-Lall.) Steud., Sandbar Lovegrass (Dorr 7959 US)

Eragrostis hirsuta (Michx.) Nees, Bigtop Lovegrass (Smith 4941 US)

[Eragrostis hirsuta var. laevivaginata Fernald]

Eragrostis hypnoides (Lam.) Britton, Sterns, \& Poggenb., Teal Lovegrass (Fosberg \& Rainey 60659 US)

- Eragrostis mexicana Link ssp. virescens (J. Presl) S. D. Koch \& Sánchez Vega, Mexican Lovegrass, † Waif (Norton s.n. MARY)

[Eragrostis neomexicana Vasey]

Eragrostis minor Host, Little Lovegrass, † (Baltars 3427 US)

[Eragrostis eragrostis (L.) P. Beauv.; E. poaeoides P. Beauv. ex Roem. \& Schult.; E. suaveolens Becker ex Claus]

Eragrostis pectinacea (Michx.) Nees var. pectinacea, Tufted Lovegrass (Beyersdorfer s.n. US)

[Eragrostis diffusa Buckley; E. purshii Schrad.]

Eragrostis pilosa (L.) P. Beauv., Indian Lovegrass, † (Killip o Swallen 32163 US)

Eragrostis refracta (Muhl.) Scribn., Coastal Lovegrass, S3S4/ G5 (Hill 15874 MARY)

[Eragrostis campestris Trin.]

Eragrostis spectabilis (Pursh) Steud., Purple Lovegrass (Harvey 7823 US)

Eragrostis trichodes Nash, Sand Lovegrass, † (Frye 6247 MARY)

- Eragrostis trichophora Cass. \& Durieu, Hairyflowered Lovegrass, † Waif (Reed 41334 US)

[Eragrostis atherstonii Stapf.]

- Eragrostis unioloides (Retz.) Nees ex Steud., Chinese Lovegrass, † Waif Reed (1964)
- Eriochloa acuminata Kunth, Tapertip Cutgrass, † Waif (Reed 32728 US)

[Eriochloa acuminata var. minor (Vasey) R. B. Shaw; E. gracilis (Fournier) Hitchc.]

- Eriochloa punctata (L.) Desv. ex Ham., Louisiana Cutgrass, † Waif Reed (1964)

Festuca filiformis Lam., Hairy Fescue, † (Frye et al. 5300 TAWES)

[Festuca ovina L. var. capillata (Lam.) Alef.]

Festuca myuros L., Annual Fescue, † (Chase 463 US)

[Vulpia myuros (L.) C. C. Gmel.]

Festuca octoflora Walter var. glauca (Nutt.) Fernald, Northern Six-weeks Grass (Cooley s.n. TAWES)

[Festuca gracilenta Buckley; F. tenella Willd.; Vulpia octoflora (Walter) Rydb.]

Festuca octoflora Walter var. octoflora, Six-weeks Fescue (Frye 805 TAWES)

[Festuca octoflora var. aristulata Torr. ex L. H. Dewey; Vulpia octoflora (Walter) Rydb. var. octoflora]

Festuca paradoxa Desv., Cluster Fescue, S1?/G5 (Carter s.n. PH)

[Festuca nutans Spreng.; F. shortii Kunth ex Alph. Wood]

Festuca rubra L., Red Fescue, † (Hermann 11891 US)

Festuca sciurea Nutt., Squirrel-tail Fescue (Hitchcock 12628 US) [Vulpia elliotea (Raf.) Fernald; V. sciurea (Nutt.) Henrard]

Festuca subverticillata (Pers.) E. B. Alexeev, Nodding Fescue (Chickering s.n. US)

[Festuca obtusa Spreng.]

Festuca trachyphylla (Hack.) R. P. Murray, Hard Fescue, † (Terrell 6090 MARY)

[Festuca brevipila R. Tracey; F. ovina L.; F. ovina var. duriuscula (L.) Koch]

Greeneochloa coarctata (Eaton) P. M. Peterson, Soreng, Romasch. \& Barberá, Nuttall's Reedgrass (Boone s.n. TAWES)

[Calamagrostis cinnoides (Muhl.) Bartram, C. coarctata (Torr.) Eaton]

Glyceria acutiflora Torr., Creeping Manna Grass, S1/G5 (Thompson s.n. TAWES)

[Panicularia acutiflora (Torr.) Kuntze]

Glyceria canadensis (Michx.) Trin., Rattlesnake Manna Grass (Hotchkiss 6327 US)

Glyceria declinata Bréb., Waxy Manna Grass (Harms 7992 US)

- Glyceria fluitans (L.) R. Br., Water Manna Grass-see Excluded Flora section.

- Glyceria grandis S. Watson, American Manna Grass, S1/G5 - see Excluded Flora section.

Glyceria laxa (Scribn.) Scribn., Lax Manna Grass (Leonard 21203 US)

[Glyceria canadensis (Michx.) Trin. var. laxa Hitchc.]

Glyceria melicaria (Michx.) F. T. Hubbard, Slender Manna Grass (Simpson s.n. US) 
Glyceria obtusa (Muhl.) Trin., Coastal Manna Grass (Naczi 8866 DOV)

Glyceria septentrionalis Hitchc., Floating Manna Grass (Killip 36572 US)

[Panicularia septentrionalis (Hitchc.) E. P. Bicknell]

Glyceria striata (Lam.) Hitchc., Fowl Manna Grass (Hitchcock 12696 US)

[Panicularia nervata (Willd.) Kuntze; Panicum striata (Lam.) Hitchc.]

Gymnopogon ambiguus (Michx.) Britton, Sterns, \& Poggenb., Bearded Skeleton Grass (Ball 80 US)

Gymnopogon brevifolius Trin., Pineland Skeleton Grass, S1/G5 (McAvoy 7122 DOV)

- Hackelochloa granularis (L.) Kuntze, Pitscale Grass, † Waif Reed (1964)

[Rytilix granularis (L.) Skeels]

Holcus lanatus L., Velvet Grass, † (Hotchkiss \& Uhler 7072 US) [Nothoholcus lanatus (L.) Nash]

- Hordeum brachyantherum Nevski ssp. brachyantherum, Meadow Barley—see Excluded Flora section.

[Hordeum nodosum L.]

Hordeum jubatum L., Foxtail Barley, † (Baltars 3118 US)

- Hordeum marinum Huds., Seaside Barley, † Waif (Reed 33404 US)

[Hordeum hystrix Roth]

- Hordeum murinum L., Mouse Barley, † Waif (Reed 33404 US) [Hordeum leporinum Link; H. murinum ssp. leporinum (Link) Arcang.]

Hordeum pusillum Nutt., Little Barley (Blake 11749 US)

Hordeum vulgare L., Common Barley, † (Cohen 8 US)

Hymenachne bemitomon (Schult.) C. C. Hsu, Maidencane, S3/G5? (Hirst s.n. DOV)

[Panicum hemitomon Schult.]

- Ischaemum indicum (Houtt.) Merr., Indian Murainagrass, † Waif Reed (1964)

[Ischaemum ciliare Retz]

- Ischaemum rugosum Salisb., Ribbed Murainagrass, † Waif Reed (1964)

Kellochloa verrucosa (Muhl.) Lizarazu, Nicola \& Scataglini, Warty Panic Grass (Knapp 947 NCU)

[Panicum verrucosum Muhl.]

- Koeleria macrantha (Ledeb.) Schult., Prairie June Grass, † Waif Reed (1964)

[Koeleria nitida Nutt.]

Leersia hexandra Sw., Southern Cut Grass, S1/G5 (Hirst 1075 DOV)

[Homalocenchrus hexandrus (Sw.) Kuntze]
Leersia lenticularis Michx., Catchfly Grass, S1/G5 (Naczi 11580 DOV)

[Homalocenchrus lenticularis (Michx.) Kuntze]

Leersia oryzoides (L.) Sw., Rice Cut Grass (Terrell 3296 US)

[Homalocenchrus oryzoides (L.) Haller]

Leersia virginica Willd., White Grass (Baltars 3222 US)

[Homalocenchrus virginicus (Willd.) Britton]

Lolium arundinaceum (Schreb.) Darbysh., Tall Fescue $†$ (Hotchkiss 5902 US)

[Festuca arundinacea Schreb.; F. elatior L.; Schedonorus arundinaceus (Schreb.) Dumort.]

Lolium perenne L., Perennial Ryegrass, † (Smith 5091 US) [Lolium multiflora Lam.; L. perenne ssp. multiflorum (Lam.) Husn.]

Lolium pratense (Huds.) Darbysh., Meadow Fescue, † (Killip \& Swallen 32125 US)

[Festuca pratensis Huds.; Schedonorus pratensis (Huds.) P. Beauv.]

Lolium temulentum L., Darnel Ryegrass, † (Longbottom 5168 DOV)

Melica mutica Walter, Two-flowered Melic, S3/G5 (Steury 030628.1 US)

Melica nitens (Scribn.) Nutt. ex Piper, Three-flowered Melic, - S2/G5 (Hill \& Cress 11826 MARY)

- Melinis repens (Willd.) Zizka, Rose Natal Grass, † Waif (Reed 41298 US)

[Rhynchelytrum repens (Willd.) C. E. Hubb.]

Microstegium vimineum (Trin.) A. Camus, Japanese Stilt Grass, ‡(Redman 5725 BALT)

[Eulalia viminea (Trin.) Kuntze; E. viminea var. variabilis Kuntze]

Milium effusum L., American Milletgrass (Frye 6364 MARY)

Miscanthus sinensis Andersson, Chinese Silvergrass, $\ddagger$ (Longbottom 14236 US)

Mnesithea rugosa (Nutt.) de Koning \& Sosef, Wrinkled Jointtail Grass, S1/G5 (Knapp 2594 TAWES)

[Coelorachis rugosa (Nutt.) Nash, Manisuris rugosa (Nutt.) Kuntze]

- Moorochloa eruciformis (Sm.) Veldkamp, Sweet Signal Grass, † Waif (Reed 32763 US)

[Brachiaria eruciformis (Sm.) Brisebach]

- Mublenbergia asperifolia (Trin.) Parodi, Scratch Grass, † Waif Reed (1964)

- Mublenbergia bushii Pohl, Nodding Muhly—see Excluded Flora section.

[Mublenbergia brachyphylla Bush] 
Mublenbergia capillaris (Lam.) Trin., Hair-awn Muhly, S1/ G5 (Holm s.n. US)

Mublenbergia frondosa (Poir.) Fernald, Wirestem Muhly (Baltars 3804 US)

Mublenbergia glabriflora Scribn., Inland Muhly, SH/G4? (Scribner s.n. US)

Mublenbergia glomerata (Willd.) Trin., Spiked Muhly, SH/G5 (Norvik s.n. MARY)

Mublenbergia mexicana (L.) Trin., Mexican Muhly (Ubler s.n. US)

- Mublenbergia racemosa (Michx.) Britton, Sterns, \& Poggenb., Marsh Muhly—see Excluded Flora section.

Mublenbergia schreberi J. F. Gmel., Nimbleweed (Dewey 106 US)

[Mublenbergia diffusa Willd.; M. schreberi var. palustris Scribn.]

Mublenbergia sobolifera (Muhl.) Trin., Rock Muhly (Simmons \&. Tice s.n. US)

Mublenbergia sylvatica (Torr.) Torr. ex A. Gray, Woodland Muhly, S3/G5 (Scribner s.n. US)

[Mublenbergia umbrosa Scribn.]

Mublenbergia tenuiflora (Willd.) Britton, Sterns, \& Poggenb., Slender Muhly (Steele s.n. US)

Mublenbergia torreyana (Schult.) Hitchc., New Jersey Muhly, - S1/G3 (Knapp 96-03 DOV)

Mublenbergia uniflora (Muhl.) Fernald, Bog Muhly (Knapp et al. 2319 US)

Oplismenus undulatifolius (Ard.) P. Beauv., Wavyleaf Basketgrass, $\ddagger$ (Imlay s.n. US)

[Oplismenus hirtellus (L.) P. Beauv. ssp. undulatifolius (Ard.) U. Scholz]

Oryzopsis asperifolia Michx., Roughleaf Ricegrass, S2/G5 (Frye et al. $6132 \mathrm{MARY}$ )

Panicum amarulum Hitchc. \& Chase, Coastal Panicgrass (Longbottom 4566 DOV)

[Panicum amarum Elliott ssp. amarulum (Hitchc. \& Chase) Freckmann \& Lelong]

Panicum amarum Elliott, Bitter Panicgrass (Chase 8504 US)

Panicum capillare L., Old Panicgrass (Beyersdorfer 358 US) [Panicum capillare var. agreste Gatt.; P. capillare var. occidentale Rydb.]

Panicum dichotomiflorum Michx. var. dichotomiflorum, Spreading Panicgrass (Hitchcock 15742 US)

[Panicum dichotomiflorum var. geniculatum (Alph. Wood) Fernald; P. proliferum Lam.]

Panicum flexile Scribn., Wiry Panicgrass, S1/G5 (Steele s.n. US)

Panicum gattingeri Nash, Gattinger's Panicgrass (Knapp 2130 $\mathrm{NCU})$
[Panicum capillare L. var. campestre Gatt.; P. philadelphicum Bernh. ex Trin. ssp. gattingeri (Nash) Freckman \& Lelong]

Panicum miliaceum L., Proso Millet, † (Novik s.n. US)

- Panicum paludosum Roxb., Marsh Panicgrass, † Waif Reed (1964)

Panicum philadelphicum Bernh. ex Trin., Philadelphia Panicgrass, SU/G5 (Knapp $1641 \mathrm{NCU})$

[Panicum capillare L. var. sylvaticum Torr.; P. tuckermanii Fernald]

- Panicum sumatrense Roth ex Roem \& Schult., Little Millet, † Waif Reed (1964)

[Panicum psilopodium Trin.]

Panicum virgatum L., Panicgrass (Baltars 3262 US)

[Panicum virgatum var. cubense Griseb.; P. virgatum var. spissum Linder]

Parapholis incurva (L.) C. E. Hubb., Curved Sicklegrass, † (Knapp $1201 \mathrm{DOV})$

Paspalum boscianum Flüggé, Bull Crowngrass (Novik s.n. MARY)

- Paspalum convexum Flüggé, Latin American Crowngrass, † Waif (Reed 32810 US)

Paspalum dilatatum Poir., Dallisgrass, $†$ (Hotchkiss 7455 US)

Paspalum dissectum (L.) L., Mudbank Crowngrass, S2/G4? (McAvoy 1302 DOV) [Paspalum membranaceum Walter]

- Paspalum distichum Michx., Knotgrass, † Waif (Reed 32749 US) [Paspalum paspaloides (Michx.) Scribn.]

Paspalum floridanum Michx., Florida Paspalum (Fosburg 18493 US)

[Paspalum difforme Leconte; P. floridanum var. glabratum Engelm. ex Vasey; P. giganteum Baldwin ex Vasey; P. glabratum (Engelm. ex Vasey) C. Mohr]

Paspalum fluitans (Elliott) Kunth, Horsetail Paspalum, S2/G5 (Killip 40546 US)

[Paspalum repens $\mathrm{P}$. J. Bergius]

Paspalum laeve Michx., Field Paspalum (Chase 2600 US) [Paspalum circulare Nash; P. laeve var. circulare (Nash) Fernald; P. laeve var. pilosum Scribn.; P. longipilum Nash]

- Paspalum orbiculatum Poir., Circular Paspalum, † Waif Reed (1964)

Paspalum pubiflorum Rupr. var. glabrum Vasey ex Scribn., Hairy-seed Paspalum (Longbottom 6619 DOV)

- Paspalum scrobiculatum L., Kodo Millet, † Waif Reed (1964)

Paspalum setaceum Michx. var. ciliatifolium (Michx.) Vasey, Ciliate Thin Paspalum (Norton s.n. MARY)

[Paspalum ciliatifolium Michx.]

Paspalum setaceum Michx. var. mublenbergii (Nash) D. J. Banks, Muhlenberg's Thin Paspalum (Novik s.n. MARY) [Paspalum calvescens Fernald; P. ciliatifolium Michx. var. mublenbergii (Nash) Fernald] 
Paspalum setaceum Michx. var. setaceum, Thin Paspalum (Novik s.n. MARY)

[Paspalum ciliatifolium Michx. var. stramineum (Nash) Fernald; P. stramineum Nash]

- Paspalum setaceum Michx. var. villosissimum (Nash) Vasey, Villose Paspalum—see Excluded Flora section.

Patis racemosa (Sm.) Romasch., P. M. Peterson \& Soreng, Blackseed Ricegrass, S2S3/G5 (Riefner 81-695 MARY)

[Oryzopsis racemosa (Sm.) Ricker ex Hitchc.; Piptatherum racemosum (Sm.) Barkworth]

Phalaris arundinacea L., Reed Canarygrass, $\ddagger$ (Beyersdorfer 111 US)

- Phalaris canariensis L., Annual Canarygrass, † Waif Reed (1964)

- Phalaris caroliniana Walter, Carolina Canarygrass-see Excluded Flora section.

Phalaris minor Rentz., Little-seed Canarygrass, $†$ (s. coll., s.n. MARY)

- Phalaris paradoxa L., Hood Canarygrass, † Waif Reed (1964)

Phleum pratense L., Timothy, † (Killip \& Swallen 31877 US)

Pbleum subulatum (Savi) Asch. \& Graebn., Italian Timothy, $\dagger($ Reed 33401 US)

Phragmites australis (Cav.) Trin. ssp. americanus Saltonst., P. M. Peterson \& Soreng, American Common Reed (Shull 276 US)

Phragmites australis (Cav.) Trin. ssp. australis, European Common Reed, $\ddagger$ (Chase 12663 US)

[Phragmites communis Trin.; P. phragmites (L.) H. Karst]

Phyllostachys aurea Carrière ex Rivière \& C. Rivière, Golden Bamboo, ‡ (Longbottom 24299 MARY)

Phyllostachys aureosulcata McClure, Yellow Groove Bamboo, † (Grant 89-00656 DOV)—perhaps persisting after planting

Phyllostachys bambusoides Siebold \& Zucc., Japanese Timber Bamboo, † (Hill \& Klein 15777 MARY)

- Phyllostachys flexuosa Rivière \& C. Rivière, Drooping Timber Bamboo-see Excluded Flora section.

Phyllostachys nigra (Lodd. ex Lindl.) Munro, Black Bamboo, † (Longbottom \& Knapp 24362 MARY)

- Piptatherum miliaceum (L.) Coss., Smilograss, † Waif Reed (1964)

[Oryzopsis miliacea (L.) Benth. \& Hook. f. ex Asch. \& Schweinf.]

Piptochaetium avenaceum (L.) Parodi, Blackseeded Speargrass (Longbottom 19309 MARY)

[Stipa avenacea L.]
Pleioblastus fortunei (Van Houtte) Nakai, Pygmy Bamboo, † (Parrish \& Steury 120308.1 US)

[Pleioblastus pygmaeus (Mig.) Nakai]

Poa alsodes A. Gray, Groove Bluegrass (Novik s.n. US)

Poa annua L., Annual Bluegrass, † (Chase 2722 US)

Poa autumnalis Muhl. ex Elliott, Autumn Bluegrass (Hermann 11457 US)

Poa bulbosa L. var. vivipara Koeler, Bulbous Bluegrass, † (Soreng 7474 US)

- Poa chapmaniana Scribn., Chapman's Bluegrass-see Excluded Flora section.

Poa compressa L., Canada Bluegrass, † (Hitchcock s.n. US)

Poa cuspidata Nutt., Early Bluegrass (Knapp 510 DOV)

Poa iconia Azn. var. iconia, Iconium Bluegrass, † (Rappleye 1552 US)

- Poa languida Hitchc., Old-pasture Bluegrass-see Excluded Flora section.

Poa nemoralis L., Wood Bluegrass, † (Kanal 609 US)

Poa palustris L., Fowl Bluegrass, S1/G5 (Soreng 9321 TAWES)

Poa pratensis L. ssp. pratensis, Kentucky Bluegrass, † (Beyersdorfer 14-84 US)

Poa pratensis ssp. angustifolia (L.) Lej., Narrow-leaved Kentucky Bluegrass, † (Kearny 70 US)

Poa saltuensis Fernald \& Wiegand, Old-pasture Bluegrass, S1/G5 (Novik s.n. US)

Poa sylvestris A. Gray, Woodland Bluegrass (Terrell 4030 US)

Poa trivialis L. ssp. sylvicola (Guss.) H. Lindb., Rough Bluegrass, † (Davis 5564 US)

Polypogon monspeliensis (L.) Desf., Annual Rabbit's-foot Grass, $\dagger$ (Freeman s.n. US)

Pseudosasa japonica (Siebold \& Zucc. ex Steud.) Makino ex Nakai, Arrow Bamboo, † (Parrish \& Steury 120308.2 US)

Puccinellia distans (Jacq.) Parl., Weeping Alkaligrass, † (Cusick $37523 \mathrm{CM}$ )

Puccinellia fasciculata (Torr.) E. P. Bicknell, Saltmarsh Alkaligrass, † (Knapp $1884 \mathrm{DOV})$

- Rostraria cristata (L.) Tzvelev, Mediterranean Hairgrass, † Waif Reed (1964)

[Koeleria pleoides Pers.]

Saccharum alopecuroides (L.) Nutt., Silver Plume Grass, S1?/ G5 (Frye et al. s.n. TAWES)

[Erianthus alopecuroides (L.) Elliott; Erianthus divaricatus (L.) Hitchc]

Saccharum baldwinii Spreng., Narrow Plume Grass, S1/G5 (McAvoy 1965 DOV)

[Erianthus strictus Baldwin] 
- Saccharum brevibarbe (Michx.) Pers. var. brevibarbe, Shortbearded Plume Grass-see Excluded Flora section.

[Erianthus brevibarbis (Michx.) Pers. var. brevibarbis]

Saccharum brevibarbe (Michx.) Pers. var. contortum (Elliott) R. D. Webster, Bent-awn Plume Grass, S3S4/G5 (McAvoy 7125 DOV)

[Erianthus brevibarbis (Michx.) Pers. var. contortus (Elliott) D. B. Ward; E. contortus Elliott; E. contortus var. compactus (Nash) Fernald; E. smallii Nash; S. contortum (Elliott) Nutt.]

Saccharum coarctatum (Fernald) R. D. Webster, Brown Plume Grass (McAvoy 3162 DOV)

[Erianthus coarctatus Fernald]

Saccharum giganteum (Walter) Pers., Giant Plume Grass (McAvoy 3155 DOV)

[Erianthus giganteus (Walter) Muhl; Erianthus saccharoides Michx.]

Sacciolepis striata Nash, American Cupscale, S1/G5 (McAvoy 1285 DOV)

[Panicum gibbum Elliott]

Schizachne purpurascens Swallen, False Melic, S1/G5 (Norton et al. s.n. MARY)

Schizachyrium littorale (Nash) E. P. Bicknell, Shore Little Bluestem (Higgins s.n. US)

[Andropogon divergens (Hack.) Andersson ex Hitchc.; A. littoralis Nash]

Schizachyrium scoparium (Michx.) Nash var. scoparium, Little Bluestem (Dewey 132 US)

[Andropogon scoparius Michx.]

Sclerochloa dura (L.) P. Beauv., Common Hardgrass, † (Cusick 37695 CM)

Secale cereale L., Rye, † (Smith 4417 US)

Setaria faberi R. A. W. Herrm., Japanese Bristlegrass, † (Baltars 3173 US)

- Setaria grisebachii E. Fourn., Grisebach's Bristlegrass, † Waif Reed (1954)

Setaria italica (L.) P. Beauv., Foxtail Millet, † (Harms 6852 US)

Setaria magna Griseb., Giant Bristlegrass (Hitchcock 7230 US)

Setaria parviflora (Poir.) Kerguélen, Marsh Bristlegrass (Baltars 3198 US)

[Setaria geniculata P. Beauv.]

Setaria pumila (Poir.) Roem. \& Schult., Yellow Foxtail, † (Baltars 3773 US)

[Setaria glauca (L.) P. Beauv.; S. lutescens (Weigel) F. T. Hubb.; S. pumila ssp. pallide-fusca (Schumach.) B. K. Simon]

Setaria verticillata (L.) P. Beauv., Hooked Bristlegrass, † (Reed 32674 US)
- Setaria verticilliformis Dumort., Barbed Bristlegrass, † Waif (Otis s.n. DOV) [Setaria verticillata (L.) P. Beauv. var. ambigua Guss.]

Setaria viridis (L.) P. Beauv., Green Bristlegrass, † (Baltars 3809 US)

Sorghastrum elliottii (C. Mohr) Nash, Slender Indiangrass, - S1/G5 (Frye 5593 TAWES)

Sorghastrum nutans (L.) Nash, Indiangrass (Chase 12560 US)

- Sorghum bicolor (L.) Moench ssp. bicolor, Sorghum, † Waif Reed (1964)

[Holcus sorghum L.; Sorghum vulgare L. var. technicum (Kornicke) Javorka]

Sorghum halepense (L.) Pers., Johnson Grass, $\ddagger$ (Longbottom 13789 US)

[Holcus halepensis L.]

Sphenopholis intermedia (Rydb.) Rydb., Slender Wedgescale (Baltars 3081 US)

[Sphenopholis obtusata (Michx.) Scribn. var. major (Torr.) Erdman]

Sphenopholis nitida (Biehler) Scribn., Shiny Wedgescale (Hitchcock 12624 US)

Sphenopholis obtusata (Michx.) Scribn., Prairie Wedgescale (Fosberg 18157 US)

[Sphenopholis obtusata var. pubescens (Scribn. \& Merr.) Scribn.]

Sphenopholis pensylvanica (L.) Hitchc., Swamp Wedgescale, S2/G4 (McAvoy 1506 DOV)

Sporobolus alterniflorus (Loisel.) P. M. Peterson \& Saarela, Smooth Cordgrass (Fosberg 34810 US)

[Spartina alterniflora Loisel.; S. stricta (Aiton) Roth var. maritima (Walter) Scribn.]

Sporobolus clandestinus (Biehler) Hitchc., Rough Dropseed, S2/G5 (McAvoy 7096 DOV)

Sporobolus compositus (Poir.) Merrill var. compositus, Tall Dropseed (Dewey 136 US)

[Sporobolus asper (P. Beauv.) Kunth var. asper]

- Sporobolus coromandelianus (Retz.) Kunth, Madagascar Dropseed, † Waif Reed (1964)

Sporobolus cynosuroides (L.) P. M. Peterson \& Saarela, Big Cordgrass (Fosberg 34809 US)

[Spartina cynosuroides (L.) Roth; S. polystachya Michx.]

Sporobolus heterolepis (A. Gray) A. Gray, Prairie Dropseed, - S1/G5 (McAvoy 703 DOV)

Sporobolus indicus (L.) R. Br., Smut Grass, † (Steury 990821.4 US)

[Sporobolus berterianus (Trin.) Hitchc. \& Chase; S. poiretii (Roem. \& Schult.) Hitchc.]

Sporobolus michauxianus (Hitchc.) P. M. Peterson \& Saarela, Prairie Cordgrass (Tidestrom 7224 US)

[Spartina pectinata (Aiton) Muhl.] 
Sporobolus neglectus Nash, Puffsheath Dropseed (Knapp 4152 TAWES)

Sporobolus pumilus (Roth) P. M. Peterson \& Saarela, Saltmeadow Cordgrass (Fosberg 18488 US)

[Spartina patens (Aiton) Muhl., S. patens var. caespitosa (A. A. Eaton) Hitchc.; S. patens var. monogyna (M. A. Curtis) Fernald]

- Sporobolus pyramidatus (Lam.) Hitchc., Whorled Dropseed, † Waif (Reed 32766 US)

Sporobolus vaginiflorus (Torr.) Alph. Wood var. vaginiflorus, Poverty Dropseed (Chase 8457 US)

- Sporobolus virginicus (L.) Kunth, Seashore Dropseed, † Waif Reed (1964)

- Themeda arguens (L.) Hack., Christmas Grass, † Waif Reed (1964)

[Themeda frondosa (R. Br.) Merr.]

Torreyochloa pallida (Torr.) G. L. Church var. fernaldii (Hitchc.) Dore, Fernald's Manna Grass, S1/G5T4Q (Frye \& Peterson 6037 TAWES)

[Glyceria pallida (Torr.) Trin. var. fernaldii Hitchc.]

Torreyochloa pallida (Torr.) G. L. Church var. pallida, Pale False Manna Grass, S3/G5 (Uhler s.n. US)

[Glyceria pallida (Torr.) Trin.; Puccinellia pallida (Torr.) R. T. Clausen]

Tragus berteronianus Schult., Spiked Bur Grass, † (Frye 6386 MARY)

Tragus racemosus (L.) All., Stalked Bur Grass, † (Knapp 4152b TAWES)

Tridens chapmanii (Small) Chase, Chapman's Purple-top, S1/ G5 (McAvoy 6927 DOV)

[Tridens flavus (L.) Hitchc. var. chapmanii (Small) Shinners]

Tridens flavus (L.) Hitchc., Purple-top (Blake 8613 US)

Tripidium ravennae (L.) H. Scholz, Ravennagrass, † (Simmons \& Bergman 3340 US)

[Erianthus ravennae (L.) P. Beauv.; Ripidium ravennae (L.) Trin.; Saccharum ravennae (L.) L.]

Triplasis purpurea (Walter) Chapm., Purple Sandgrass (Chase 12646 US)

[Sieglingia purpurea (Walter) Kuntze; Triplasis intermedia Nash]

Tripsacum dactyloides (L.) L., Eastern Gamagrass (Blake s.n. US)

Triticum aestivum L., Common Wheat, † (Smith 4416 US)
Uniola paniculata L., Seaoats, † (Hamilton 543 Assateague Island National Seashore Herbarium)

- Urochloa distachya (L.) T. Q. Nguyen, Tropical Signalgrass, † Waif Reed (1964)

[Brachiaria subquadripara (Trin.) Hitchc.; Urochloa subquadripara (Trin.) R. D. Webster]

- Urochloa mutica (Forssk.) T. Q. Nguyen, Para Grass, † Waif Reed (1964)

[Panicum purpurascens Raddi]

- Urochloa panicoides P. Beauv., Panic Liverseed Grass, † Waif Reed (1964)

Urochloa plantaginea (Link) R. D. Webster, Plantain Signalgrass, † (Terrell et al. 5580 MARY) [Brachiaria plantaginea (Link) Hitchc.]

Urochloa platyphylla (Munro ex Wright) R. D. Webster, Broadleaf Signalgrass, † (Longbottom 22022 TAWES) [Brachiaria platyphylla (Munro ex Wright) Nash]

- Urochloa ramosa (L.) T. Q. Nguyen, Browntop Millet, † Waif Reed (1964)

[Brachiaria ramosa (L.) Stapf; Panicum ramosum L.]

- Urochloa reptans (L.) Stapf, Sprawling Signalgrass, † Waif Reed (1964)

[Brachiaria reptans (L.) C. A. Gardner \& C. E. Hubb.; Panicum prostratum Lam.; P. reptans L.]

Urochloa texana (Buckley) R. D. Webster, Texas Signalgrass, † (Longbottom 22023 TAWES)

[Brachiaria texana (Buckley) S. T. Blake; Panicum texanum Buckley]

- Urochloa villosa (Lam.) T. Q. Nguyen, Hairy Signalgrass, † Waif Reed (1964)

[Brachiaria distichophylla (L.) Stapf]

Zea mays L., Maize, † (Hill 8921 MARY)

- Zea mexicana (Schrad.) Kuntz, Mexican Teosinte-see Excluded Flora section.

Zizania aquatica L. var. aquatica, Annual Wildrice (Naczi 8869 DOV)

- Zizania palustris L. var. palustris, Northern Wildrice-see Excluded Flora section.

[Zizania aquatica L. var. angustifolia Hitchc.]

Zizaniopsis miliacea (Michx.) Döll \& Asch., Giant Cutgrass, - S1/G5 (McAvoy 1284 DOV)

Zoysia japonica Steud., Korean Lawngrass, † (Terrell 4434 MARY)

PODOSTEMACEAE (Riverweed Family)

Podostemum ceratophyllum Michx., Threadfoot, S3/G5 (Hotchkiss 7107 US)

[Podostemum abrotanoides Nutt.] 


\section{POLEMONIACEAE}

(Phlox Family)

Ipomopsis rubra (L.) Wherry, Red Standing-cypress, † (Tatnall 394 DOV)

[Gilia coronopifolia (Willd.) Pers.; G. rubra (L.) A. Heller]

- Linanthus pungens (Torr.) J. M. Porter \& L. A. Johnson, Granite Prickly Phlox, † Waif Reed (1964)

[Leptodactyon pungens (Torr.) Rydb.]

- Phlox amplifolia Britton, Largeleaf Phlox, † Waif (Reed $26509 \mathrm{MO})$

- Pblox carolina L., Thickleaf Phlox-see Excluded Flora section.

[Phlox carolina ssp. angusta Wherry; P. carolina ssp. turritella Wherry]

Phlox divaricata L. var. divaricata, Wild Blue Phlox (Tatnall 4466 DOV)

[Phlox divaricata var. laphamii Alph. Wood]

Phlox drummondii Hook., Drummond's Phlox, † (Fontes 45 MARY)

Phlox glaberrima L., Smooth Phlox, S1/G5 (Parish s.n. US) [Phlox glaberrima ssp. interior (Wherry) Wherry; P. glaberrima ssp. triflora (Michx.) Wherry]

Phlox maculata L., Spotted Phlox (Hotchkiss 5344 US) [Phlox maculata ssp. pyramidalis (Sm.) Wherry]

- Phlox ovata L., Mountain Phlox, SH/G4-see Excluded Flora section. [Phlox latifolia Michx.]

Phlox paniculata L., Fall Phlox (Hitchcock 15627 US)

Phlox pilosa L., Downy Phlox, S1/G5 (Wherry s.n. US) [Phlox pilosa ssp. detonsa (A. Gray) Wherry]

Phlox stolonifera Sims, Creeping Phlox (Longbottom et al. 4177 DOV)

[Phlox reptans Michx.]

Phlox subulata L., Moss Phlox (Isanogle 48 US)

[Phlox subulata var. brittonii Small]

Polemonium reptans L. var. reptans, Jacob's-ladder (Olds s.n. US)

Polemonium vanbruntiae Britton, Bog Jacob's-ladder, S2/ G3G4 (Knapp et al. 1389 DOV)

\section{POLYGALACEAE}

(Milkwort Family)

- Polygala brevifolia Nutt., Littleleaf Milkwort-see Excluded Flora section.

Polygala cruciata L., Crossleaf Milkwort, S2/G5 (Knapp 103-03 TAWES)

[Polygala cruciata var. aquilonia Fernald \& B. G. Schub.; P. ramosior (Nash) Small]

Polygala curtissii A. Gray, Curtiss' Milkwort (Frye 960821-638 TAWES)

- Polygala cymosa Walter, Tall Pinebarren Milkwort-see Excluded Flora section.
Polygala incarnata L., Pink Milkwort, S2S3/G5 (Knapp \& Stango 61-03 TAWES)

Polygala lutea L., Yellow Milkwort (Frye \& Harrison s.n. TAWES)

[Pylostachya lutea (L.) Small]

Polygala mariana Mill., Maryland Milkwort (Strong \& Simmons 1974 US)

[Polygala harperi Small]

Polygala nuttallii Torr. \& A. Gray, Nuttall's Milkwort (Frye 3149 TAWES)

- Polygala paniculata L., Orosne-see Excluded Flora section.

Polygala polygama Walter, Racemed Milkwort, S1/G5 (Killip 40536 US)

- Polygala ramosa Elliott, Low Pinebarren Milkwort-see Excluded Flora section.

Polygala sanguinea L., Field Milkwort (Frye 980916-04 TAWES) [Polygala viridescens L.]

Polygala senega L. var. latifolia Torr. \& A. Gray, Broad-leaved Seneca Snakeroot, S2/G4G5 (Knapp 3575 NY)

Polygala senega L. var. senega, Seneca Snakeroot, S2/G4G5 (Frye \& Baranski 1046 TAWES)

Polygala verticillata L., Whorled Milkwort (Isanogle 70 US) [Polygala ambigua Nutt.; P. pretzii Pennell; P. verticillata var. ambigua (Nutt.) Alph. Wood; P. verticillata var. isocycla Fernald]

Polygaloides paucifolia (Willd.) J. R. Abbott, Gaywings (Buker s.n. CM)

[Triclisperma pancifolia Nieuwl.; Polygala panciflora Willd.]

POLYGONACEAE

(Buckwheat Family)

Fagopyrum esculentum Moench, Buckwheat, † (Leonard 19907 US)

[Fagopyrum sagittatum Gilib.]

- Fallopia baldschuanica (Regel) Holub, China Fleece-vine-see Excluded Flora section.

[Polygonum baldschuanicum Regel]

Fallopia cilinodis (Michx.) Holub, Fringed Black Bindweed, - S3/G5 (Jennings $6272 \mathrm{CM}$ )

[Polygonum cilinode Michx.]

Fallopia convolvulus (L.) Á. Löve, Black Bindweed, † (Longbottom et al. 9872 DOV)

[Polygonum convolvulus L.]

Fallopia cristata (Engelm. \& A. Gray) Holub, Climbing False Buckwheat (Norton s.n. MARY)

[Polygonum scandens L. var. cristatum (Engelm. \& A. Gray) Gleason]

- Fallopia dumetorum (L.) Holub, Thicket Buckwheat-see Excluded Flora section.

[Polygonum scandens L. var. dumetorum (L.) Gleason]

Fallopia scandens (L.) Holub, Climbing False Buckwheat (Frye \& Peterson 6041 TAWES)

[Polygonum scandens L. var. scandens] 
Persicaria amphibia (L.) Delarbre, Water Smartweed (Frye 6074 TAWES)

[Persicaria amphibia var. emersa (Michx.) J. C. Hickman; Polygonum amphibium L.; P. coccineum Muhl. ex Willd.; P. mublenbergii (S. Watson) Small]

Persicaria arifolia (L.) Haraldson, Halberd-leaf Tearthumb (Thompson 7965 CM)

[Polygonum arifolium L.]

- Persicaria careyi (Olney) Greene, Carey's Smartweed-see Excluded Flora section.

[Polygonum careyi Olney]

- Persicaria chinensis (L.) H. Gross, Chinese Knotweed-see Excluded Flora section.

Persicaria extremiorientalis (Vorosch.) Tzvelev, Far-eastern Smartweed, † (Longbottom 12318 NY)

Persicaria filiformis (Thunb.) Nakai, Painted Knotweed, $\dagger$ (Fulton s.n. US)

Persicaria glabra (Willd.) M. Gómez, Smooth Knotweed (Longbottom 4472 DOV)

[Persicaria densiflora (Meisn.) Moldenke, Polygonum densiflorum Meisn.; P. portoricense Bert. ex Endl.]

Persicaria hydropiper (L.) Opiz, Marshpepper Smartweed, † (Grant 89-00530 DOV)

[Polygonum hydropiper L.]

Persicaria hydropiperoides (Michx.) Small, Mild Water-pepper (Frye 960726-600 TAWES)

[Persicaria opelousana (Riddell ex Small) Small; Polygonum bydropiperoides Michx.; P. opelousanum Riddell ex Small]

Persicaria lapathifolia (L.) Delarbre, Dock-leaf Smartweed, $\ddagger$ (Longbottom 10683 DOV)

[Polygonum incarnatum Elliott; P. lapathifolia L.]

Persicaria longiseta (Bruijn) Moldenke, Creeping Smartweed, † (Thompson $6460 \mathrm{CM})$

[Polygonum cespitosum Blume var. longisetum (Bruijm) A. N. Steward]

Persicaria maculosa Gray, Spotted Lady's-thumb, † (Longbottom 10629 DOV)

[Persicaria persicaria (L.) Small; Polygonum dubium Stein ex A. Braun; P. maculatum Raf.; P. persicaria L.; P. puritanorum Fernald]

Persicaria orientalis (L.) Spach, Garden's Gate, † (O’Neil s.n. DOV)

[Polygonum orientale L.]

Persicaria pensylvanica (L.) M. Gómez, Pennsylvania Smartweed (Frye 960826-640 TAWES)

[Persicaria longistyla Small; P. mississippiensis (Stanford) Small; Polygonum pensylvanicum L.; P. pensylvanicum var. rosaeflorum Norton]

Persicaria perfoliata (L.) H. Gross, Mile-a-minute Weed, $\ddagger$ (Longbottom 12209 DOV)

[Polygonum perfoliatum L.]

Persicaria punctata (Elliott) Small, Dotted Smartweed (McAvoy 7044 DOV)

[Polygonum punctatum Elliott]
Persicaria robustior (Small) E. P. Bicknell, Stout Smartweed, SU/G4G5 (Longbottom 7862 DOV)

[Polygonum robustius (Small) Fernald]

Persicaria sagittata (L.) H. Gross, Arrowleaf Tearthumb (Longbottom 10656 DOV)

[Polygonum sagittatum L.; Tracaulon sagittatum (L.) Small]

Persicaria setacea (Baldwin) Small, Bog Smartweed, SU/G5 (Longbottom 7799 DOV)

[Polygonum setaceum Baldwin]

Persicaria virginiana (L.) Gaertn., Virginia Knotweed (Longbottom 7939 DOV)

[Polygonum virginianum L.; Tovara virginiana (L.) Raf.]

Polygonella articulata (L.) Meisn., Eastern Jointweed (Cooley s.n. TAWES)

Polygonum aviculare L., Oval-leaf Knotweed, † (O’Neil s.n. DOV)

[Polygonum arenastrum Boreau; P. articulatum (L.) Meisn.; P. aviculare ssp. bellardii (All.) Kuntze; P. aviculare L. ssp. depressum Meisn.; P. bellardii All.]

- Polygonum douglasii Greene, Douglas' Knotweed, † Waif Reed (1964)

Polygonum erectum L., Erect Knotweed (O’Neil s.n. DOV)

Polygonum glaucum Nutt., Seabeach Knotweed, S1/G3 (Frye 5114 TAWES)

[Polygonum maritimum L.]

Polygonum prolificum (Small) B. L. Rob., Proliferous Knotweed (Norton s.n. MARY)

[Polygonum exsertum Small; P. ramosissimum Michx. var. prolificum Small]

Polygonum ramosissimum Michx., Bushy Knotweed, SH/G5 (O’Neil s.n. DOV)

Polygonum tenue Michx., Slender Knotweed (Knapp 3395 DOV)

Reynoutria japonica Houtt., Japanese Knotweed, $\ddagger$ (Baltars 784 US)

[Fallopia japonica (Houtt.) Ronse Decr.; Polygonum cuspidatum Siebold \& Zucc.]

Reynoutria sachalinensis (F. Schmidt) Nakai, Giant Knotweed, † (Lea 3355 DOV)

[Polygonum sachalinense F. Schmidt]

Rumex acetosella L., Sheep Sorrel, † (Hitchcock 12765 US) [Acetosella acetosella (L.) Small]

Rumex altissimus Alph. Wood, Tall Dock, S1/G5 (Hitchcock 12549 US)

Rumex crispus L., Curly Dock, † (Fleming 2149 US)

- Rumex fueginus Phil., Sea-side Dock-see Excluded Flora section.

[Rumex maritimus L.]

Rumex hastatulus Baldwin ex Elliott, Heartwing Sorrel (Hall 21 MARY) 
Rumex obtusifolius L., Bitter Dock, $\uparrow$ (Hotchkiss 5730 US)

Rumex patientia L., Patience Dock, † (Steele s.n. US)

Rumex pulcher L., Fiddle Dock, † (Blake 11748 US)

- Rumex sanguineus L., Redvein Dock-see Excluded Flora section.

Rumex verticillatus L., Swamp Dock (Leonard 22059a US) [Rumex floridanus Meisn.]

\section{PONTEDERIACEAE}

(Water Hyacinth Family)

Heteranthera dubia (Jacq.) MacMill., Grassleaf Mudplantain (Tidestrom $7636 \mathrm{DOV}$ )

[Zosterella dubia (Jacq.) Small]

Heteranthera multiflora (Griseb.) Horn, Bouquet Mudplantain (Tatnall $4946 \mathrm{GH}$ )

Heteranthera pauciflora C. N. Horn, Few-flowered Mudplantain (Long $41098 \mathrm{PH}$ )

Heteranthera reniformis Ruiz \& Pav., Kidneyleaf Mudplantain (McAvoy 1707 DOV)

Pontederia cordata L., Pickerelweed (Longbottom 9361 DOV) [Pontederia lanceolata Nutt.]

Pontederia crassipes Mart., Common Water Hyacinth, $\ddagger$ (Knapp 2341 TAWES)

[Eichhornia crassipes (Mart.) Solms]

PORTULACACEAE

(Purslane Family)

Portulaca amilis Speg., Paraguayan Purslane, † (Longbottom $26780 \mathrm{NY}$ )

- Portulaca grandiflora Hook., Moss Rose, † Waif (Trox s.n. MARY)

Portulaca oleracea L., Common Purslane, $\ddagger$ (Leonard 20981 US)

POTAMOGETONACEAE

(Pondweed Family)

Potamogeton amplifolius Tuck., Largeleaf Pondweed, S1S2/ G5 (Uhler \& Nelson s.n. US)

Potamogeton bicupulatus Fernald, Snailseed Pondweed (Frye $6581 \mathrm{NY}$ )

Potamogeton crispus L., Curly Pondweed, $¥$ (Killip 13456 US)

Potamogeton diversifolius Raf., Waterthread Pondweed (Baltars 648 US)

[Potamogeton capillaceus Poir.]

Potamogeton epibydrus Raf., Ribbonleaf Pondweed (Hotchkiss 5491 US)

Potamogeton foliosus Raf., Leafy Pondweed, S2/G5 (Killip 13367 US)

Potamogeton gramineus L., Grassy Pondweed (Hall \& Knapp JH2014.09.10-21 MARY)

Potamogeton illinoensis Morong, Illinois Pondweed, S2/G5 (Commons s.n. PH)

[Potamogeton angustifolius Bercht. \& J. Presl; P. heterophyllus Schreb.; P. lucens L.]

Potamogeton natans L., Floating Pondweed, † (Carter s.n. PH)
Potamogeton nodosus Poir., Longleaf Pondweed (Beyersdorfer 351 US)

[Potamogeton americanus Cham. \& Schltdl.; P. fluitans Roth; P. lonchites Tuck.]

- Potamogeton obtusifolius Mert. \& W. J. D. Koch, Bluntleaf Pondweed-see Excluded Flora section.

- Potamogeton praelongus Wulfen, Whitestem Pondweed-see Excluded Flora section.

Potamogeton perfoliatus L., Claspingleaf Pondweed, S3/G5 (Uhler \& Martin s.n. US)

[Potamogeton perfoliatus var. bupleuroides (Fernald) Farw.]

Potamogeton pulcher Tuck., Spotted Pondweed (Hotchkiss 7973 US)

Potamogeton pusillus L. ssp. pusillus, Dwarf Slender Pondweed, - S2S4/G5 (Hirst 909 DOV)

[Potamogeton pusillus var. minor (Biv.) Fernald \& B. G. Schub.]

Potamogeton pusillus L. ssp. tenuissimus (Mert. \& W. D. J. Koch) R. R. Haynes \& Hellq., Slender Pondweed (Moldenke 9398 NY)

[Potamogeton berchtoldii Fieber var. lacunatus (Hagst.) Fernald]

- Potamogeton richardsonii (A. Benn.) Rydb., Richardson's Pondweed-see Excluded Flora section.

Potamogeton robbinsii Oakes, Robbins' Pondweed, S1?/G5 (Shull 165 US)

- Potamogeton spirillus Tuck., Spiral Pondweed, SU/G5-see Excluded Flora section.

Potamogeton vaseyi J. W. Robbins, Vasey's Pondweed (Hall JH2014.09.24-01 MARY)

Potamogeton zosteriformis Fernald, Flatstem Pondweed, S1/G5 (Shull 163 US)

Stuckenia pectinata (L.) Börner, Sago Pondweed (Killip 32214 US) [Potamogeton pectinatus L.]

Zannichellia palustris L., Horned Pondweed (Ward s.n. US)

PRIMULACEAE (Primrose Family)

Hottonia inflata Elliott, Featherfoil, S1/G4 (Steury 980529.2 US)

Lysimachia arvensis (L.) U. Manns \& Anderb., Scarlet Pimpernel, $†$ (Beard s.n. MARY)

[Anagallis arvensis L. ssp. arvensis]

Lysimachia borealis (Raf.) U. Manns \& Anderb., Northern Starflower (McAvoy 2255 DOV)

[Trientalis borealis Raf.]

Lysimachia ciliata L., Fringed Loosestrife (Hotchkiss 5355 US) [Steironema ciliatum (L.) Baudo]

Lysimachia foemina (Mill.) U. Manns \& Anderb., Blue Pimpernel, $†$ (Beard s.n. MARY)

[Anagallis arvensis L. var. caerulea Arechav.; A. arvensis ssp. foemina (Mill.) Schinz \& Thell.] 
Lysimachia bybrida Michx., Lanceleaf Loosestrife, S2/G5 (Steury 980720.5 US)

[Steironema bybridum (Michx.) Raf. ex Small]

Lysimachia japonica Thunb., Japanese Yellow Loosestrife, $\dagger$ (Davis 7243A BALT)

Lysimachia lanceolata Walter, Lanceleaf Loosestrife, S3/G5 (Baltars 664 US)

[Steironema heterophyllum (Michx.) Baudo; S. lanceolatum (Walter) A. Gray]

Lysimachia maritima (L.) Galasso, Banfi \& Soldano, Sea Milkwort, SH/G5 (Tatnall 3105 DOV)

[Glaux maritima L.]

Lysimachia minima (L.) U. Manns \& Anderb., Chaffweed, - SU/G5 (Steele s.n. US)

[Anagallis minima (L.) E. H. L. Krause; Centunculus minimus L.]

Lysimachia nummularia L., Creeping Jenny, † (Hotchkiss 5407 US)

- Lysimachia punctata L., Spotted Loosestrife, † Waif [Shull s.n. US)

Lysimachia quadriflora Sims, Four-flowered Yellow Loosestrife (Farnsworth 1335MD CM)

Lysimachia quadrifolia L., Whorled Yellow Loosestrife (Hotchkiss 5241 US)

Lysimachia terrestris (L.) Britton, Sterns, \& Poggenb., Swamp Loosestrife (Fosberg 17119 US)

Lysimachia thyrsiflora L., Water Loosestrife, S1/G5 (Steury $82584 \mathrm{PH})$

Lysimachia vulgaris L., Garden Loosestrife, † (Steury 990710.5 US)

Primula meadia (L.) A. R. Mast \& Reveal, Common Shootingstar, S3/G5 (Frye s.n. MARY)

[Dodecatheon meadia L.]

- Primula veris L., Cowslip Primrose, † Waif (O’Neill s.n. MARY)

Samolus valerandi L., Water Pimpernel (Kearney 37 US)

[Samolus floribundus Kunth; S. parviflorus Raf; S. valerandi ssp. parviflorus (Raf.) Hultén]

\section{RANUNCULACEAE (Buttercup/Crowfoot Family)}

Aconitum uncinatum L., Blue Monkshood, S1/G4 (Olds s.n. US) [Aconitum uncinatum var. acutidens Fernald; A. uncinatum var. muticum DC.]

Actaea pachypoda Elliott, White Baneberry (Leonard 1345 US) [Actaea alba Mill.]

Actaea podocarpa DC., Mountain Bugbane, S2/G4 (Hill $9533 b$ MARY)

[Cimicifuga americana Michx.]

Actaea racemosa L., Black Cohosh (Hitchcock 12797 US)

[Cimicifuga racemosa (L.) Nutt.]
Anemone acutiloba (DC.) G. Lawson, Sharp-lobed Hepatica (Hedge 22 MARY)

[Hepatica acutiloba DC.]

Anemone americana (DC.) H. Hara, Roundleaf Liverleaf (Carter 26 BALT)

[Hepatica americana (DC.) Ker Gawl.]

Anemone canadensis L., Canada Anemone, SH/G5 (Steele s.n. US)

[Anemonidium canadense (L.) Á. Löve \& D. Löve]

Anemone lancifolia Pursh, Mountain Thimbleweed, S1?/G5 (Boone s.n. MARY)

- Anemone minima DC., Nightcaps-see Excluded Flora section.

Anemone quinquefolia L., Wood Anemone (McAvoy1341 DOV)

- Anemone virginiana L. var. alba (Oakes) Alph. Wood, Virginia Anemone-see Excluded Flora section.

Anemone virginiana L. var. virginiana, Tall Thimbleweed (McAvoy 1666 DOV)

Aquilegia canadensis L., Wild Columbine (Knapp 505 DOV)

- Aquilegia vulgaris L., European Columbine, † Waif (Ditman s.n. MARY)

Caltha palustris L., Marsh Marigold (Knapp 2757 DOV)

Clematis occidentalis (Hornem.) DC. var. occidentalis, Purple Clematis, S1/G5T5 (Boone s.n. MARY)

[Clematis verticillaris DC.]

Clematis ochroleuca Aiton, Curlyheads, SH/G4-see Reveal et al. (1987) (H.S. [Sloane] 14.05 MARY)

Clematis terniflora DC., Japanese Virgin's-bower, $\ddagger($ Knapp 2646 DOV)

[Clematis dioscoreifolia Leveille \& Vaniot; C. paniculata Thunb.]

Clematis viorna L., Vase-vine Leatherflower, S3/G5 (Killip 13702 US)

Clematis virginiana L., Virginia Virgin's-bower (Strong et al. 1792 US)

Clematis vitalba L., Evergreen Clematis, † (Baltars 351 US)

Consolida ajacis (L.) Schur, Doubtful Knight's-spur, † (McAtee 1629 US)

[Consolida ambigua (L.) P. W. Ball \& Heywood; Delphinium ajacis $\mathrm{L}$.

- Consolida regalis Gray, Royal Larkspur-see Excluded Flora section.

[Delphinium consolida L.]

Coptis trifolia (L.) Salisb. var. groenlandica (Oeder) Fassett, Goldthread, S1/G5T5 (Boone s.n. TAWES)

Delphinium exaltatum Aiton, Tall Larkspur, S1/G3 (Frye $6393 \mathrm{MO})$

[Delphinium urceolatum Jacq.]

Delphinium tricorne Michx., Dwarf Larkspur, S3/G5 (Leonard 2522 US) 
Eranthis hyemalis (L.) Salisb., Winter Aconite, † (Miller s.n. MARY)

Ficaria verna Huds., Fig Buttercup, $\ddagger$ (Redman 6320 BALT) [Ficaria verna ssp. calthifolia (Rchb.) Nyman; F. verna ssp. chrysocephala (P. D. Sell) Stace; F. verna ssp. fertilis (Laegaard) Stace; F. verna ssp. ficariiformis (Rouy \& Foucaud) B. Walln; Ranunculus ficaria L. var. bulbifera Albert; R. ficaria ssp. calthifolius (Rchb.) Arcang.]

- Helleborus xhybridus hort. ex Vilmorin, Hellebore, † Waif (Hill Photo Voucher MPA)

Helleborus viridis L., Green Hellebore, $†$ (Smith 58 BALT)

Hydrastis canadensis L., Goldenseal, S2/G3G4 (Smith 67 BALT)

Myosurus minimus L., Tiny Mousetail (Goff 021 MARY)

Nigella damascena L., Devil-in-the-bush, † (Kilgus s.n. MARY)

Ranunculus abortivus L., Kidneyleaf Buttercup (Knapp 512 DOV)

Ranunculus acris L., Tall Buttercup, † (Hotchkiss 5842 US)

Ranunculus allegheniensis Britton, Allegheny Mountains Crowfoot, S3/G4G5 (Downs 2663 NCU)

Ranunculus ambigens S. Watson, Water-plantain Spearwort, S1/G4 (Hermann 11539 US)

[Ranunculus obtusiusculus Raf.]

- Ranunculus arvensis L., Corn Buttercup-see Excluded Flora section.

Ranunculus bulbosus L., Bulbous Buttercup, † (Terrell s.n. US)

Ranunculus caricetorum Greene, Northern Swamp Buttercup (Frye \& Lea 5065 TAWES)

[Ranunculus hispidus var. caricetorum (Greene) T. Duncan]

Ranunculus fascicularis Muhl. ex Bigelow, Early Buttercup, - S1/G5 (Wolff s.n. BALT)

Ranunculus flabellaris Raf., Yellow Water Crowfoot, S1/G5 (Cook s.n. MARY)

[Ranunculus delphiniifolius Torr. ex Eaton]

Ranunculus hederaceus L., Long-stalked Crowfoot, S1/G5 (Knapp 2398 TAWES)

Ranunculus hispidus Michx. var. hispidus, Bristly Buttercup (Hirst \& Wilson 1005 DOV)

Ranunculus hispidus Michx. var. nitidus (Elliott) T. Duncan, Hispid Buttercup, S1S3/G5T5 (Knapp 3219 TAWES)

[Ranunculus carolinianus DC.; R. palmatus Elliott; R. septentrionalis Poir.]

Ranunculus laxicaulis (Torr. \& A. Gray) Darby, Mississippi Buttercup, S1/G5? (Knapp 1939 TAWES)

Ranunculus micranthus Nutt., Rock Crowfoot (Steury 970330 US)

Ranunculus parviflorus L., Small-flowered Buttercup, † (Naczi 11337 DOV)
Ranunculus pensylvanicus L., Bristly Crowfoot, SH/G5-a now extirpated species formerly known from Druid Hill Park, Baltimore County, which is also the historic location of other rare plants such as Coptis trifolia.

A specimen supposedly exists at the Cylburn Arboretum, but we were not able to examine it. Given the reports of reputable botanists who saw this in the field before the population was destroyed, it is retained here on the checklist.

Ranunculus pusillus Poir., Low Spearwort, SU/G5 (Hotchkiss 5077 US)

[Ranunculus lindheimeri Engelm.; R. oblongifolius Elliott; R. pusillus var. lindheimeri (Engelm.) A. Gray]

Ranunculus recurvatus Poir., Hooked Crowfoot (Killip 36872 US)

Ranunculus repens L., Creeping Buttercup, † (Hotchkiss 5841 US)

Ranunculus sardous Crantz, Hairy Buttercup, † (Knapp \& McAvoy)

[Ranunculus parvulus L.]

Ranunculus sceleratus L. var. sceleratus, Cursed Crowfoot (Leonard 21124 US)

- Ranunculus trichophyllus Chaix var. trichophyllus, Threadleaf Water Crowfoot, S1/G5—see Excluded Flora section. [Ranunculus aquatilis L.]

Thalictrum amphibolum Greene, Waxyleaf Meadow-rue (Hitchcock 12702 US)

[Thalictrumpurpurascens L.; T. revolutum DC.-misapplied]

Thalictrum coriaceum (Britton) Small, Leatherleaf Meadow-rue (Terrell 4110 US)

[Thalictrum steeleanum B. Bovin]

- Thalictrum dasycarpum Fischer \& Avé-Lall., Purple Meadowrue-see Excluded Flora section.

Thalictrum dioicum L., Early Meadow-rue (Maxon 6095 US)

- Thalictrum macrostylum Small \& A. Heller, Small-Leaved Meadow-rue-see Excluded Flora section.

[Thalictrum subrotundum B. Boivin]

Thalictrum pubescens Pursh, Tall Meadow-rue (Arsène 810 US)

[Thalictrum perelegans Greene; T. polygamum Muhl. ex Spreng.]

Thalictrum thalictroides (L.) B. Boivin, Windflower (Russell 361 BALT)

[Anemonella thalictroides (L.) Spach; Syndesmon thalictroides (L.) Hoffm. ex G. Lawson]

Trautvetteria caroliniensis (Walter) Vail, Carolina Tassel-rue, - S3/G5 (Knapp \& Thompson 3218 MARY)

Xanthorbiza simplicissima Marshall, Shrubby Yellowroot, † (Fessenden \& Brandenburg 5505 US)

[Xanthorbiza apiifolia L'Hér.] 


\section{RESEDACEAE}

(Mignonette Family)

Reseda lutea L., Yellow Upright Mignonette, † (Wherry s.n. US)

- Reseda luteola L., Dyer's Rocket, † Waif Reed (1964)

RHAMNACEAE

(Buckthorn Family)

- Berchemia scandens (Hill) K. Koch, Supplejack-see Excluded Flora section.

Ceanothus americanus L., New Jersey Tea (Knapp 2794 DOV)

Frangula alnus Mill., Glossy False Buckthorn, † (Terrell s.n. MARY)

[Rhamnus frangula L.]

Frangula caroliniana (Walter) A. Gray, Carolina Buckthorn, ‡(Wortbley s.n. MARY)

[Rhamnus caroliniana Walter]

Rhamnus alnifolia L'Her., Alderleaf Buckthorn, S1/G5 (McPherson s.n. TAWES)

- Rhamnus cathartica L., Common Buckthorn-see Excluded Flora section.

Rhamnus davurica Pall., Dahurian Buckthorn, ‡ (Choe 013 NA)

- Rhamnus lanceolata Pursh ssp. lanceolata, Lanceleaf Buckthorn-see Excluded Flora section

\section{ROSACEAE}

(Rose Family)

Agrimonia gryposepala Wallr., Tall Hairy Groovebur (McAvoy $5224 \mathrm{DOV})$

Agrimonia microcarpa Wallr., Small-fruited Agrimony, S1?/ G5 (McAvoy 1149 DOV)

[Agrimonia platycarpa Wallr.; A. pubescens Wallr. var. microcarpa (Wallr.) H. E. Ahles; A. pumila Muhl. ex E. P. Bicknell]

Agrimonia parviflora Aiton, Swamp Agrimony (McAvoy 1197 DOV)

Agrimonia pubescens Wallr., Soft Groovebur (McAvoy 6625 DOV)

[Agrimonia bicknellii (Kearney) Rydb.; A. mollis (Torr. \& A. Gray) Britton]

Agrimonia rostellata Wallr., Beaked Agrimony (McAvoy 1254 DOV)

Agrimonia striata Michx., Woodland Agrimony, S1/G5 (Boone s.n. TAWES)

Amelanchier arborea (Michx.) Fernald, Downy Serviceberry (Knapp 12-03 TAWES)

Amelanchier canadensis (L.) Medik., Oblong-leaved Serviceberry (Knapp $2652 \mathrm{DOV})$

Amelanchier humilis Wiegand, Low Serviceberry, S1/G5 (Frye \& Roberts 5495 TAWES)

Amelanchier intermedia Spach, Intermediate Serviceberry (Norton et al. s.n. MARY)
Amelanchier laevis Wiegand, Allegheny Serviceberry (Frye \& Roberts 5497 TAWES)

[Amelanchier arborea (Michx.) Fernald ssp. laevis (Wiegand)

S. M. McKay ex P. Landry]

Amelanchier nantucketensis E. P. Bicknell, Nantucket Shadbush, - S1/G5 (Frye \& Harrison 5027 TAWES)

Amelanchier obovalis (Michx.) Ashe, Obovate-leaved Coastal Juneberry (Harrison s.n. TAWES)

Amelanchier sanguinea (Pursh) DC., Roundleaved Serviceberry, - S1/G5 (Frye s.n. TAWES)

Amelanchier spicata (Lam.) K. Koch, Running Serviceberry, S2/G5 (Frye \& Thompson s.n. TAWES)

[Amelanchier stolonifera Wiegand]

Aphanes australis Rydb., Slender Parsley Piert, † (Longbottom 1607 DOV)

- Aphanes microcarpa (Boiss. \& Reut.) Rothm, Slender Parsley Piert-see Excluded Flora section. [Alchemilla microcarpa Boiss. \& Reut.]

- Argentina anserina (L.) Rydb., Silverweed Cinquefoil-see Excluded Flora section.

[Potentilla anserina L.]

Aronia arbutifolia (L.) Pers., Red Chokeberry (Knapp 2664 DOV)

[Photinia pyrifolia (Lam.) K. R. Robertson \& J. B. Phipps; Pyrus arbutifolia (L.) L. f.]

Aronia melanocarpa (Michx.) Elliott, Black Chokeberry (Baltars 597 US)

[Photinia melanocarpa (Michx.) K. R. Robertson \& J. B. Phipps; Pyrus melanocarpa (Michx.) Willd.]

Aronia prunifolia (Marshall) Rehder, Purple Chokeberry, S3/ G4G5Q (McAvoy 6146 DOV)

[Aronia arbutifolia (L.) Pers. var. atropurpurea (Britton) F. Seym.; A. atropurpurea Britton; Photinia floribunda (Lindl.) K. R. Robertson \& J. B. Phipps; Pyrus floribunda Lindl.]

Aruncus dioicus (Walter) Fernald, Common Goat's-beard (Killip 31047 US)

[Aruncus allegheniensis Rydb.]

Chaenomeles japonica (Thunb.) Lindl. ex Spach, Maule's Quince, † (Hill 9832 MARY)

- Chaenomeles speciosa (Sweet) Nakai, Flowering Quince, † Waif (Hill 9826 MARY)

Crataegus calpodendron (Ehrh.) Medik., Pear Hawthorn (Palmer 39557 US)

[Crataegus tomentosa L.]

Crataegus chrysocarpa Ashe, Fireberry Hawthorn (Reveal \& Broome 4633 MARY)

[Crataegus evansiana Sarg.; C. mercerensis Sarg.; C. sicca Sarg.] 
Crataegus coccinea L. var. coccinea, Scarlet Hawthorn (Penson s.n. MARY)

[Crataegus pedicellata Sarg.; C. pennsylvanica Ashe; C. tatnalliana Sarg.]

Crataegus crus-galli L. var. crus-galli, Cockspur Hawthorn (Fosberg 43700 US)

[Crataegus arborea Beadle; C. canbyi Sarg.; C. crus-galli var. pyacanthifolia Aiton.; C. mohrii Beadle; C. signata Beadle]

Crataegus dodgei Ashe, Dodge's Hawthorn (Palmer 39557a US)

Crataegus intricata Lange, Copenhagen Hawthorn (Buker s.n. $\mathrm{CM})$

[Crataegus biltmoreana Beadle; C. boyntonii Beadle; C. contrita Beadle; C. inanis Beadle; C. mendosa Beadle; C. pinetorum Beadle; C. sargentii Beadle]

- Crataegus macracantha Lodd. ex Loudon, Long-thorned Hawthorn-see Excluded Flora section.

Crataegus macrosperma Ashe, Big-fruit Hawthorn (Henry s.n. CM)

[Crataegus flabellata (Bosc ex Spach) K. Koch]

- Crataegus margarettae Ashe, Margarett's Hawthorn, see Excluded Flora section.

Crataegus monogyna Jacq. var. monogyna, English Hawthorn, † (Meyer 21516 TRT)

Crataegus phaenopyrum (L. f.) Medik., Washington Hawthorn (Norris 364 CM)

[Crataegus populifolia Walter]

- Crataegus populnea Ashe, Poplar Hawthorn-see Excluded Flora section.

[Crataegus iracunda Beadle var. populnea (Ashe) Kruschke]

Crataegus pruinosa (H. L. Wendl.) K. Koch, Waxy-fruit Hawthorn (Shurr 22 MARY)

[Crataegus gattingeri Ashe; C. pruinosa var. dissona (Sarg.) Eggl.; C. rugosa Ashe]

Crataegus punctata Jacq., Dotted Hawthorn (Norton s.n. MARY)

[Crataegus punctata var. aurea Aiton; C. punctata var. canescens Britton; C. punctata var. microphylla Sarg.]

Crataegus rubella Beadle, Reddish Hawthorn (Hotchkiss 5661 US)

- Crataegus schuettei Ashe var. schuettei, Schuette's Hawthornsee Excluded Flora section.

[Crataegus alnorum Sarg.; C. basilica Beadle; C. ferrissii Ashe; C. silvicola Beadle; C. stolonifera Sarg.; C. tortilis Ashe]

Crataegus succulenta Schrad. ex Link var. succulenta, Fleshy Hawthorn (Norton s.n. MARY)

Crataegus uniflora Münchh., Dwarf Hawthorn (Buker s.n. CM) [Crataegus armentalis Beadle]

Crataegus viridis L. var. viridis, Green Hawthorn (Ebert \& Holt $128 \mathrm{DOV})$

[Crataegus blanda Sarg.; C. lanceolata Sarg.; C. penita Beadle; C. vulsa Beadle]
Cydonia oblonga Mill., Common Quince, † (Hillegeist s.n. MARY)

Dalibarda repens L., Robin Runaway (Frye 2099 TAWES) [Rubus repens (L.) Kuntze]

Exochorda racemosa (Lindl.) Rehder, Common Pearlbush, $\dagger$ (s. coll., s.n. MARY)

Filipendula rubra (Hill) B. L. Rob., Queen of the Prairie, - S1/G4G5 (Fessenden 5252 US)

Fragaria vesca L. var. vesca, Woodland Strawberry (Reveal \& Broome 4669 MARY)

Fragaria virginiana Duchesne, Virginia Strawberry (Allard 19796 US)

[Fragaria australis (Rydb.) Rydb.]

Geum aleppicum Jacq., Yellow Avens, S1/G5 (Killip 31074 US) [Geum aleppicum var. strictum (Aiton) Fernald; G. strictum Aiton]

Geum canadense Jacq., White Avens (Hitchcock s.n. US)

Geum fragarioides (Michx.) Smedmark, Barren Strawberry (Henry s.n. CM)

[Waldsteinia fragarioides (Michx.) Tratt. ssp. fragarioides]

Geum laciniatum Murray, Rough Avens, S3/G5 (Leonard 19924 US)

[Geum laciniatum var. trichocarpum Fernald]

Geum rivale L., Purple Avens, SH/G5 (Stone 8646 PH)

Geum vernum (Raf.) Torr. \& A. Gray, Spring Avens (Steury 970429.2 US)

[Stylypus vernus Raf.]

Geum virginianum L., Pale Avens (Steele s.n. US) [Geum hirsutum Muhl. ex Link]

- Gillenia stipulata (Muhl. ex Willd.) Nutt., American Ipecacsee Excluded Flora section.

[Porteranthus stipulatus (Muhl. ex Willd.) Britton]

Gillenia trifoliata (L.) Moench, Bowman's-root (Bartsch s.n. US) [Porteranthus trifoliatus (L.) Britton]

Malus angustifolia (Aiton) Michx., Southern Crabapple, S3/G5? (Boone s.n. TAWES)

[Pyrus angustifolia Aiton]

- Malus baccata (L.) Borkh., Siberian Crabapple-see Excluded Flora section.

Malus coronaria (L.) Mill., Sweet Crabapple (Tatnall 2825 DOV)

[Malus bracteata Sarg.; M. glabrata Rehder; M. glaucescens Rehder; M. lancifolia Rehder; Pyrus coronaria L.]

- Malus ioensis (Alph. Wood) Britton var. ioensis, Prairie Crabapple-see Excluded Flora section. 
Malus prunifolia (Willd.) Borkh., Plumleaf Crabapple, † (House 1517 US)

Malus pumila Mill., Paradise Apple, † (Hall s.n. MARY) [Malus domestica (Suckow) Borkh.; M. sylvestris Mill.; Pyrus malus L.; P. pumila (Mill.) Steud.]

Malus toringo (Siebold) Siebold ex de Vriese, Toringo Crabapple, $†$ (Terrell 5870 MARY)

[Malus sieboldii (Regel) Rehder; Pyrus sieboldii Regel]

Photinia villosa (Thunb.) DC., Oriental Photinia, † (Davis Photo Voucher MPA)

Physocarpus opulifolius (L.) Maxim., Eastern Ninebark (Hitchcock 12774 US)

Potentilla argentea L., Silvery Cinquefoil, † (Otis s.n. DOV)

Potentilla arguta Pursh, Tall Cinquefoil, SH/G5 (Baltars 1520 US)

[Drymocallis arguta (Pursh) Rydb.]

Potentilla canadensis L. var. canadensis, Canada Cinquefoil (Fosberg 42315 US)

[Potentilla canadensis var. villosissima Fernald; P. caroliniana Poir.; P. pumila Poir.]

Potentilla indica (Andrews) Th. Wolf, Indian Mock Strawberry, $\dagger$ (Leonard 21848 US)

[Duchesnea indica (Andrews) Teschem.]

Potentilla intermedia L., Downy Cinquefoil, † (Stieber s.n. NA)

Potentilla norvegica L., Norwegian Cinquefoil (Hotchkiss 5314 US)

[Potentilla monspeliensis L.; P. norvegica ssp. monspeliensis (L.) Asch. \& Grab.]

Potentilla recta L., Sulphur Cinquefoil, † (Smith 5208 US)

Potentilla reptans L., Creeping Cinquefoil, † (Fosberg 42309 US)

Potentilla rivalis Nutt., Brook Cinquefoil, † (Stieber s.n. NA)

Potentilla simplex Michx., Common Cinquefoil (Greiner s.n. US)

Poterium annuum (Nutt. ex Hook.) Spach, Prairie Burnet, $\dagger$ (Brown s.n. MARY)

[Sanguisorba annua (Nutt. ex Hook.) Torr. \& A. Gray]

Poterium sanguisorba L. ssp. muricatum Bonnier \& Layens, Salad Burnet, † (Blake 11357 US)

[Sanguisorba minor Scop.]

Prunus alleghaniensis Porter, Allegheny Plum, S2/G4 (Thompson s.n. TAWES)

Prunus americana Marshall, American Plum (Fisher s.n. US)

Prunus angustifolia Marshall, Chickasaw Plum (Leonard 21793 US)

Prunus avium L., Sweet Cherry, † (Fosberg 43584 US)

Prunus cerasus L., Sour Red Cherry, † (Vasey s.n. US)

Prunus domestica L., European Plum, † (O’Neill s.n. MARY) [Prunus domestica var. insititia (L.) Fiori \& Paol.; P. insititia L.]
Prunus hortulana L. H. Bailey, Hortulan Plum, † (Frye \& Roberts 5499 TAWES)

Prunus mahaleb L., Perfumed Cherry, † (Wheeler s.n. US)

Prunus maritima Marshall, Beach Plum, S1/G4 (Hill 14049 TAWES)

Prunus pensylvanica L., Fire Cherry (Riefner 81-72 MARY)

Prunus persica (L.) Batsch, Peach, † (Hotchkiss 7034 US)

Prunus pumila L. var. susquehanae (Willd.) H. Jaeger, Susquehanna Cherry, SH/G5 (Steele s.n. US)

[Prunus susquehanae Willd.]

Prunus rivularis Scheele, Creek Plum, † (Leonard 20161 US)

[Prunus munsoniana W. Wright \& Hedrick]

Prunus serotina Ehrh., Black Cherry (Beyersdorfer 8-84 US) [Prunus serotina var. neomontana (Small) Sudw.]

Prunus spinosa L., Blackthorn, † (Ward s.n. US)

Prunus tomentosa Thunb., Nanking Cherry, † (Hill 16842 MARY)

Prunus virginiana L., Choke Cherry (Reveal et al. 5351 MARY)

Pyracantha coccinea Roem., Scarlet Firethorn, † (Leonard 21055 US)

Pyrus calleryana Decne., Bradford Pear, $\ddagger$ (Yost 489 DOV)

Pyrus communis L., Common Pear, † (Killip 31177 US)

Pyrus pyrifolia (Burm. f.) Nakai, Chinese Pear, † (Norton s.n. MARY)

Rhodotypos scandens (Thunb.) Makino, Jetbead, $\ddagger($ Dutton 4406 MARY)

Rosa blanda Aiton, Smooth Rose, S1/G5 (Knapp \& Thompson 3217 TAWES)

Rosa bracteata J. C. Wendl., McCartney Rose, $\ddagger$ (Baltars 2452 US)

Rosa canina L., Dog Rose, † (Leonard 6689 US)

Rosa carolina L. var. carolina, Carolina Rose (Knapp 2719 DOV)

[Rosa bumilis Marshall; R. lyonii Pursh; R. serrulata Raf.]

Rosa eglanteria L., Sweetbrier, † (Leonard 1962 US) [Rosa rubiginosa L.]

Rosa gallica L., French Rose, † (Hotchkiss 7004 US)

- Rosa luciae Franch. \& Rochebr., Memorial Rose-see Excluded Flora section.

Rosa micrantha Borrer, Small-flowered Sweetbrier, † (Chickering s.n. US)

Rosa multiflora Thunb., Multiflora Rose, $\ddagger$ (Beyersdorfer 112 US)

Rosa palustris Marshall, Swamp Rose (Fleming 2240 US) [Rosa floridana Rydb.; R. lancifolia Small]

Rosa rugosa Thunb., Rugose Rose, † (Hill 13773 Assateague Island National Seashore Herbarium)

Rosa setigera Michx., Prairie Rose, † (Baltars 1175 US)

Rosa virginiana Mill., Virginia Rose (Roller 554 US) 
Rubus allegheniensis Porter, Allegheny Blackberry (Leonard \& Hotchkiss $3188 \mathrm{CM}$ )

[Rubus alumnus L. H. Bailey; R. flavinanus Blanch.; R. nigrobaccus L. H. Bailey; R. rosa L. H. Bailey]

Rubus bifrons Vest, Himalayan Berry, † (Davis 7251 Patuxent River Naval Air Station)

Rubus canadensis L., Smooth Blackberry (Downs 6887 Frostburg State University Herbarium)

Rubus cuneifolius Pursh, Sand Blackberry (Bridwell s.n. US) [Rubus longii Fernald; R. probabilis L. H. Bailey]

Rubus flagellaris Willd., Northern Dewberry (Davis \& Davis 10914 CM)

[Rubus aboriginum Rydb.; R. baileyanus Britton; R. centralis L. H. Bailey; R. curtipes L. H. Bailey; R. decar L. H. Bailey; R. depavitus L. H. Bailey; R. enslenii Tratt.; R. fecundus L. H. Bailey; R. invisus (L. H. Bailey) Britton; R. ithacanus L. H. Bailey; R. leviculus L. H. Bailey; R. michiganensis (F. W. Card ex L. H. Bailey) L. H. Bailey; R. montensis L. H. Bailey; R. multifer L. H. Bailey; R. obvius L. H. Bailey; R. plicatifolius Blanch.; R. recurvicaulis Blanch.; R. roribaccus (L. H. Bailey) Rydb.; R. scambia L. H. Bailey; R. steelei L. H. Bailey]

Rubus hispidus L., Bristly Dewberry (Leonard 21206 CM) [Rubus adjacens Fernald; R. davisiorum L. H. Bailey; R. missouricus L. H. Bailey; R. paganus L. H. Bailey; R. pervarius (L. H. Bailey) L. H. Bailey; R. provincialis L. H. Bailey]

Rubus idaeus L. var. strigosus (Michx.) Maxim., Common Red Raspberry, † (Norton s.n. MARY)

Rubus laciniatus Willd., Evergreen Blackberry, † (Baltars 722 US)

Rubus occidentalis L., Black Raspberry (Hermann 11626 US)

Rubus odoratus L., Purple-flowering Raspberry (Utech 911167 CM)

[Rubacer odoratus (L.) Rydb.]

Rubus pascuus L. H. Bailey, Chesapeake Blackberry, † (Norton s.n. MARY)

Rubus pensilvanicus Poir., Pennsylvania Blackberry (Leonard $21530 \mathrm{CM})$

[Rubus andrewsianus Blanch.; R. argutus Link; R. argutus var. floridus (Tratt.) L. H. Bailey; R. betulifolius Small; $R$. defectionsis Fernald; R. floridus Tratt.; R. frondosus Bigelow; R. grimesii L. H. Bailey; R. heterophyllus Willd.; R. immanus L. H. Bailey; R. laudatus A. Berger; R. originalis L. H. Bailey; R. ostryigolius Rydb.; R. philadelphicus Blanch; R. recurvans Blanch.; R. rhodophyllus Rydb.; R. rosarius L. H. Bailey; R. suus L. H. Bailey]

Rubus phoenicolasius Maxim., Wineberry, † (Blake s.n. US)

Rubus pubescens Raf., Dwarf Red Blackberry (Frye \& Baranski $8139 \mathrm{NY}$ )

Rubus setosus Bigelow, Small Bristleberry (Davis \& Davis 8449 CM)

[Rubus notatus L. H. Bailey; R. racemiger L. H. Bailey]

- Rubus trivialis Michx., Southern Dewberry-see Excluded Flora section.
[Rubus continentalis (Focke) L. H. Bailey; R. rubrisetus Rydb.]

Sanguisorba canadensis L., Canada Burnet, S2/G5 (Hotchkiss 5736 US)

Sibbaldiopsis tridentata (Aiton) Rydb., Three-toothed Cinquefoil, † (Porter 20 MARY)

[Potentilla tridentata Aiton]

Sorbus americana Marshall, American Mountain Ash, S3/G5 (Knapp 2841 DOV)

[Pyrus americana (Marshal) DC.]

Sorbus aucuparia L., European Mountain Ash, † (Schmid 20 MARY)

Spiraea alba Du Roi, Narrowleaf White Meadowsweet (Hotchkiss 5391 US)

Spiraea corymbosa Raf., Corymbed Spiraea, S3/G5 (Roller 641 US)

[Spirea betulifolia Pall. var. corymbosa (Raf.) Maxim.]

Spiraea japonica L., Japanese Spiraea, † (Leonard 22048 US)

Spiraea latifolia (Aiton) Borkh., Broadleaf Meadowsweet (Boone s.n. TAWES)

Spiraea prunifolia Siebold \& Zucc., Bridal-wreath, † (Standley 11341 US)

Spiraea thunbergii Siebold ex Blume, Thunberg's Meadowsweet, † (Killip 30877 US)

Spiraea tomentosa L., Steeplebush (Hitchcock 13038.5 US)

RUBIACEAE

(Madder/Coffee Family)

Cephalanthus occidentalis L., Common Buttonbush (Boone s.n. TAWES)

Diodia teres Walter, Rough Buttonweed (Killip 32183 US) [Diodella teres (Walter) Small; D. teres var. hirsutior Fernald \& Griscom; Hexasepalum teres (Walter) J. H. Kirkbr.]

Diodia virginiana L., Larger Buttonweed (Steury 980702 US) [Diodia hirsuta Pursh; D. tetragona Walter; D. virginiana var. latifolia Torr. \& A. Gray]

Galium aparine L., Catchweed Bedstraw (Leonard \& Hotchkiss 3505 US)

Galium arvense (L.) F. J. Herm., Blue Woodruff, † (Goldberg s.n. US)

[Asperula arvensis L.]

Galium asprellum Michx., Rough Bedstraw (Maxon 6383 US)

Galium boreale L., Northern Bedstraw, S1/G5 (Ebert \& Holt s.n. TAWES)

Galium circaezans Michx., Wild Licorice (Naczi 8062 DOV) [Galium circaezans var. hypomalacum Fernald]

Galium concinnum Torr. \& A. Gray, Shining Bedstraw (Lea 953 TAWES) 
Galium hispidulum Michx., Coast Bedstraw, S1/G5 (Hill 15618 Assateague Island National Seashore Herbarium) [Galium bermudense L.]

Galium lanceolatum Torr., Torrey's Wild Licorice (Baltars 2725 US)

Galium latifolium Michx., Purple Bedstraw, S3/G5 (Reveal 5533 MARY)

Galium mollugo L., Great Hedge Bedstraw, † (Hyacinth 1542 US)

Galium obtusum Bigelow, Bluntleaf Bedstraw (Boone s.n. TAWES)

[Galium obtusum var. filifolium (Wiegand) Fernald]

- Galium odoratum (L.) Scop., Sweetscented Bedstraw-see Excluded Flora section. [Asperula odorata L.]

- Galium palustre L., Marsh Bedstraw-see Excluded Flora section.

Galium pedemontanum (Bellardi) All., Piedmont Bedstraw, † (Longbottom 9131 DOV) [Cruciata pedemontana (Bellardi) Ehrend.]

Galium pilosum Aiton, Hairy Bedstraw (Frye s.n. TAWES) [Galium pilosum var. puncticulosum (Michx.) Torr. \& A. Gray]

Galium tinctorium L. var. floridanum Wiegand, Stiff Marsh Bedstraw (Walker 5582 MARY)

Galium tinctorium L. var. tinctorium, Stiff Marsh Bedstraw (Arsène 459 US)

[Galium claytonii Michx.]

- Galium tricornutum Dandy, Roughfruit Corn Bedstraw, † Waif Reed (1964)

- Galium trifidum L. var. trifidum, Threepetal Bedstraw-see Excluded Flora section.

Galium triflorum Michx., Sweet-scent Bedstraw (Boone s.n. TAWES)

- Galium uniflorum Michx., Oneflower Bedstraw—see Excluded Flora section.

Galium vernum L., Yellow Spring Bedstraw, † (Baltars 2688 US)

Houstonia caerulea L., Quaker Ladies (Fosberg 30896 US) [Hedyotis caerulea (L.) Hook.]

- Houstonia lanceolata (Poir.) Britton, Venus' Pride-see Excluded Flora section.

[Houstonia purpurea (L.) Torr. \& A. Gray var. calycosa A. Gray]

Houstonia longifolia Gaertn. var. compacta Terrell, Longleaf Bluet (Kunsman 7094 CM)

[Hedyotis longifolia (Gaertn.) Hook.; H. nuttalliana Fosberg]

- Houstonia longifolia Gaertn. var. longifolia, Longleaf Bluetsee Excluded Flora section.

Houstonia micrantha (Shinners) Terrell, Southern Bluet, † (Longbottom \& Hall 2737 NY)

Houstonia purpurea (L.) Torr. \& A. Gray var. purpurea, Purple Bluet (Beyersdorfer 65 US)

[Hedyotis purpurea (L.) Torr. \& A. Gray]
Houstonia pusilla Schöpf, Tiny Bluet (Baltars 3062 US)

[Hedyotis crassifolia Raf.; H. minima (L. C. Beck) Torr. \& A. Gray; H. minor (Michx.) Britton; H. patens Elliott]

Houstonia serpyllifolia Michx., Michaux's Bluet, S3/G4? (Boone s.n. MARY)

Houstonia tenuifolia Nutt., Slender-leaf Bluet, S1/G4G5 (Pennell $2437 \mathrm{PH}$ )

[Hedyotis longifolia (Gaertn.) Hook. var. tenuifolia (Nutt.) Torr. \& A. Gray; Houstonia longifolia Gaertn var. tenuifolia (Nutt.) Alph. Wood]

Mitchella repens L., Partridgeberry (Chase 6892 US)

Oldenlandia uniflora L., Clustered Bluets, S3/G5 (Smith 5131 US)

[Edrastima uniflora (L.) Raf.; Hedyotis uniflora (L.) Lam.]

Richardia scabra L., Rough Mexican Clover, † (Leonard 17567 US)

Sherardia arvensis L., Blue Field Madder, † (Longbottom 11361 US)

[Galium sherardia (L.) E. H. L. Krause]

Spermacoce glabra Michx., Smooth False Buttonweed, S1/ G4G5 (Wiegand Photo Voucher TAWES)

RUPPIACEAE

(Ditch Grass Family)

Ruppia maritima L., Widgeongrass (Smith 4591 US)

RUTACEAE

(Citrus/Rue Family)

Phellodendron amurense Rupr., Amur Corktree, $\ddagger$ (Davis 3596 US)

[Phellodendron japonicum Maxim.]

Poncirus trifoliata (L.) Raf., Hardy Orange, $†$ (Flory s.n. DOV) [Citrus trifoliata L.]

Ptelea trifoliata L. var. trifoliata, Common Hoptree, S3/G5 (Frye et al. 5933 TAWES)

- Ruta graveolens L., Common Rue-see Excluded Flora section.

Tetradium daniellii (Benn.) T. G. Hartley, Bee Bee Tree, † (Johnson Photo Voucher MPA)

Zanthoxylum americanum Mill., Northern Prickly-ash, S1S2/ G5 (Parrish s.n. TAWES)

[Xanthoxylum americanum Mill.—orthographic variant]

SALICACEAE

(Willow Family)

Populus alba L., White Poplar, † (Leonard 6581 US)

Populus balsamifera L., Balsam Poplar, † (Ward s.n. US) 
Populus deltoides Marshall, Eastern Cottonwood (Hotchkiss 12967 US)

Populus grandidentata Michx., Large-tooth Aspen (Leonard 6586 US)

Populus heterophylla L., Swamp Cottonwood (Uhler \& Hotchkiss 8224 US)

Populus nigra L., Black Cottonwood, † (Flory s.n. DOV)

[Populus nigra var. italica Du Roi]

Populus tremuloides Michx., Quaking Aspen (Hotchkiss 7074 US)

Salix alba L., White Willow, † (Norton s.n. MARY)

[Salix alba var. vitellina (L.) Stokes]

- Salix amygdaloides Andersson, Peachleaf Willow-see Excluded Flora section.

Salix babylonica L., Weeping Willow, † (Leonard 19775 US)

Salix bebbiana Sarg., Bebb's Willow, SH/G5 (Long 8035 PH)

- Salix caprea L., Goat Willow-see Excluded Flora section.

Salix caroliniana Michx., Carolina Willow, S3/G5 (Allard 11078 US)

[Salix longipes Shuttlew. ex Andersson; S. marginata Wimm ex Andersson.; S. wardii (Bebb) Bebb]

Salix cinerea L., European Gray Willow, † (Leonard 19195 US)

Salix discolor Muhl., Pussy Willow, SH/G5 (Redman 531 NCU)

- Salix elaeagnos Scop., Elaeagnus Willow-see Excluded Flora section.

Salix eriocephala Michx., Heartleaf Willow (Ward s.n. US)

[Salix cordata Muhl.; S. rigida Muhl.]

Salix exigua Nutt. var. sericans (Nees) Dorn, Narrowleaf Willow, S1/G5 (Ball \& Griggs 61 US)

[Salix interior Rowlee]

Salix fragilis L., Crack Willow, † (Leonard 157 US)

Salix humilis Marshall, Prairie Willow (Ball 103 US)

Salix lucida Muhl., Shining Willow, SH/G5 (Long \& Bartram $1329 \mathrm{PH})$

Salix nigra Marshall, Black Willow (Ball 2605 US)

Salix occidentalis Walter, Dwarf Prairie Willow, S2/G4G5 (Dowell 6521 US)

[Salix humilis Marshall var. microphylla (Andersson) Fernald; S. humilis var. tristis (Aiton) Griggs; S. tristis Aiton]

Salix pentandra L., Laurel Willow, † (McAtee 3391 US)

Salix purpurea L., Basket Willow, † (Westerfield 1910 CM)

Salix sericea Marshall, Silky Willow (Ball 104 US)

- Salix triandra L., Almond Willow-see Excluded Flora section.

\section{SANTALACEAE}

(Sandalwood Family)

Comandra umbellata (L.) Nutt., Umbellate Bastard Toadflax (Knapp 2666 DOV)

Phoradendron leucarpum (Raf.) Reveal \& M. C. Johnst., American Mistletoe (Hermann 11409 US)

[Phoradendron flavescens (Pursh) Nutt.; P. serotinum (Raf.) M. C. Johnst.]
SAPINDACEAE

(Maple Family)

- Acer campestre L., Hedge Maple-see Excluded Flora section.

Acer negundo L., Box Elder (McAvoy 3382 DOV) [Negundo negundo (L.) H. Karst.]

Acer nigrum F. Michx., Black Maple (Boone s.n. TAWES] [Acer saccharum L. ssp. nigrum (F. Michx.) Desmarais; Saccharodendron nigrum (F. Michx.) Small]

Acer palmatum Thunb., Japanese Maple, † (Johnson Photo Voucher MPA)

Acer pensylvanicum L., Striped Maple (Reveal et al. 5342 MARY)

Acerplatanoides L., Norway Maple, $\ddagger$ (Longbottom 7850 DOV)

Acer pseudoplatanus L., Sycamore Maple, † (Knowlton s.n. US)

Acer rubrum L., Red Maple (Norton 625 MARY)

[Acer rubrum var. trilobum Torr. \& A. Gray ex K. Koch; Rufacer carolinianum (Walter) Small; R. rubrum (L.) Small]

Acer saccharinum L., Silver Maple (McAvoy 5021 DOV)

[Argentacer saccharinum (L.) Small]

Acer saccharum Marshall var. saccharum, Sugar Maple (Kearney 93 US)

[Acer saccharum var. schneckii Rehder; Saccharodendron saccharum (Marshall) Moldenke]

Acer spicatum Lam., Mountain Maple (Frye 5175 TAWES)

Aesculus flava Sol., Yellow Buckeye, † (Thomas s.n. MARY) [Aesculus octandra Marshall]

Aesculus glabra Willd., Ohio Buckeye, †? (Lemanski s.n. MARY)

Aesculus bippocastanum L., Horse Chestnut, † (Pratt s.n. NCU)

Aesculus pavia L., Red Buckeye, † (Riefner 80315 MARY)

Aesculus parviflora Walter, Bottlebrush Buckeye, † (Harms 9240 MARY)

Koelreuteria paniculata Laxm., Golden Rain Tree, † (Partain 2008118 BALT)

SAPOTACEAE

(Sapodilla Family)

- Sideroxylon lycioides L., Buckthorn Bully-see Excluded Flora section.

[Bumelia lycioides (L.) Pers.]

SARRACENIACEAE

(Pitcher Plant Family)

Sarracenia flava L., Yellow Pitcher Plant, † (Wixted Photo Voucher TAWES)

Sarracenia purpurea L., Northern Pitcher Plant, S2/G5 (Naczi 10349 NY)

[Sarracenia purpurea var. venosa (Raf.) Fernald]

SAURURACEAE

(Lizard's-tail Family)

- Houttuynia cordata Thunb., Chameleon Plant-See Excluded Flora section.

Saururus cernuus L., Lizard's-tail (Beyersdorfer s.n. US) 


\section{SAXIFRAGACEAE}

(Saxifrage Family)

- Astilbe biternata (Vent.) Britton, False Goat's Beard-see Excluded Flora section.

Chrysosplenium americanum Schwein. ex Hook., American Golden Saxifrage (Chase 6903 US)

Heuchera alba Rydb., White Alumroot (Brighton Photo Voucher MPA)

Heuchera americana L., American Alumroot (Beyersdorfer 12-84 US)

[Heuchera calycosa Small; H. curtisii Torr. \& A. Gray; H. lancipetala Rydb.]

Heuchera hispida Pursh, American Alumroot (Brown \& Brown s.n. MARY)

[Heuchera americana L. var. hispida (Pursh) E. F. Wells]

Heuchera pubescens Pursh, Downy Alumroot (Eifrig s.n. CM)

- Heuchera villosa Michx. var. villosa, Hairy Alumroot-see Excluded Flora section.

[Heuchera macrorhiza Small]

Micranthes micranthidifolia (Haw.) Small, Lettuceleaf Saxifrage, S3/G5 (Reveal et al. 5348 MARY)

[Saxifraga micranthidifolia (Haw.) Steud.]

Micranthes pensylvanica (L.) Haw., Swamp Saxifrage (Leonard 2267 US)

[Saxifraga pensylvanica L.]

- Micranthes petiolaris (Raf.) Brouillet \& Gornall, Michaux's Saxifrage-see Excluded Flora section.

[Hydatica petiolaris (Raf.) Small; Saxifraga michauxii Britton]

Micranthes virginiensis (Michx.) Small, Virginia Saxifrage (Chase 6866 US)

[Saxifraga virginiensis Michx.]

Mitella diphylla L., Two-leaf Bishop's Cap (Smith s.n. US)

Tiarella cordifolia L., Heartleaf Foamflower (Benedict 5935 US) [Tiarella cordifolia var. collina Wherry; T. wherryi Lakela]

\section{SCROPHULARIACEAE}

(Figwort Family)

Buddleja davidii Franch., Orange-eye Butterfly-bush, † (Killip 36704 US)

Scrophularia lanceolata Pursh, Hare Figwort, S3/G5 (Frye 4048 TAWES)

Scrophularia marilandica L., Carpenter's Square Figwort (Knapp 2959 DOV)

Verbascum blattaria L., White Moth Mullein, † (Redman 832 BALT)

Verbascum lychnitis L., White Mullen, † (Fissiden 5565 US)
Verbascum phlomoides L., Clasping-leaf Mullein, † (Freeman s.n. US)

Verbascum sinuatum L., Mediterranean Mullein, † (Baltars 637 US)

Verbascum thapsus L., Common Mullein, $\ddagger$ (Markell 114 BALT)

SIMAROUBACEAE

(Quassia Family)

Ailanthus altissima (Mill.) Swingle, Tree of Heaven, $\ddagger$ (Beyersdorfer s.n. US)

SMILACACEAE

(Greenbrier Family)

Smilax bona-nox L., Saw Greenbriar, S3/G5 (Leonard \& Leonard 6684 US)

Smilax ecirrata (Engelm. ex Kunth) S. Watson, Upright Carrionflower, S1S3/G5 (Cass 80-88 BALT)

Smilax glauca Walter, Cat Greenbrier (Hitchcock 12709 US) [Smilax glauca var. leurophylla S. F. Blake]

Smilax herbacea L., Smooth Carrionflower (Blake s.n. US)

Smilax laurifolia L., Laurel Greenbrier (Knapp 539 DOV)

Smilax pseudochina L., Bamboo Vine, S2/G4G5 (Norton 278 US)

Smilax pulverulenta Michx., Downy Carrionflower (Long $37279 \mathrm{PH})$

[Smilax herbacea L. var. pulverulenta (Michx.) A. Gray]

Smilax rotundifolia L., Roundleaf Greenbrier (Coville 905 US)

Smilax tamnoides L, Bristly Greenbrier (Knapp 2849 DOV) [Smilax hispida Muhl. ex Torr.]

Smilax walteri Pursh, Red-berried Greenbrier (Knapp 538 DOV)

SOLANACEAE

(Nightshade/Potato Family)

Calibrachoa parviflora (Juss.) D’Arcy, Seaside Petunia, † (Taylor s.n. US)

[Petunia parviflora Juss.]

- Capsicum annuum L., Cayenne Pepper, † Waif Reed (1964) [Capsicum annuum var. glabriusculum (Dunal) Heiser \& Pickersgill; C. baccatum L.; C. frutescens L.]

Datura inoxia Mill., Angel's Trumpet, $\dagger$ (Baltars 4160 US)

- Datura quercifolia Kuntze, Chinese Thorn Apple, † Waif Reed (1964)

Datura stramonium L., Jimsonweed, † (Beyersdorfer 126 US) [Datura ferox L.; D. stramonium var. tatula (L.) Torr.]

- Datura wrightii Regel, Sacred Thorn Apple, † Waif Reed (1964)

[Datura meteloides auct. p.p. non Dunal]

Hyoscyamus niger L., Black Henbane, † (Hermann 11613 US)

- Jaltomata procumbens (Cav.) J. L. Gentry, Creeping False Holly, † Waif Reed (1964)

[Saracha procumbens (Cav.) Ruiz \& Pav.] 
Lycium barbarum L., Common Matrimony Vine, † (Hyacinth 959 US)

[Lycium halimifolium Mill.]

Lycium chinense Mill., Chinese Desert Thorn, † (Esher s.n. US)

Nicandra physalodes (L.) Pers., Apple of Peru, † (Killip 31947 US) [Physalodes physalodes (L.) Britton]

- Nicotiana alata Link \& Otto, Jasmine Tobacco, † Waif (Leonard 20053 US)

- Nicotiana glauca Graham, Tree Tobacco, † Waif Reed (1964)

- Nicotiana longiflora Cav., Long-flowered Tobacco-see Excluded Flora section.

- Nicotiana obtusifolia M. Mertens \& Galeotti, Desert Tobacco, † Waif Reed (1964)

[Nicotiana trigonophylla Dunal]

- Nicotiana plumbaginifolia Viv., Tex-Mex Tobacco, † Waif Reed (1964)

- Nicotiana tabacum L., Cultivated Tobacco, † Waif (Hill 9583 MARY)

Petunia xatkinsiana D. Don ex Loudon, Garden Petunia, † (Killip 3664 US)

[Petunia axillaris (Lam.) Britton, Sterns, \& Poggenb.]

Petunia integrifolia (Hook.) Schinz. \& Thell., Violetflower Petunia, † (Ward s.n. US)

Physalis alkekengi L., Strawberry Ground Cherry, † (Harms 6634 MARY)

[Alkekengi officinarum Mill.]

Physalis angulata L., Cutleaf Ground Cherry (Longbottom 14585 MARY)

- Physalis grisea (Waterf.) M. Martínez, Strawberry Tomatosee Excluded Flora section.

Physalis heterophylla Nees, Clammy Ground Cherry (Hyacinth 382 US)

[Physalis ambigua (A. Gray) Britton; P. heterophylla var. nyctaginea (Dunal) Rydb.; P. nyctaginea Dunal; P. sinuata Rydb.]

Physalis longifolia Nutt. var. subglabrata (Mack. \& Bush) Cronquist, Longleaf Ground Cherry (Hitchcock 15648 US) [Physalis subglabrata Mack. \& Bush]

Physalis philadelphica Lam., Mexican Ground Cherry, † (Hall s.n. MARY)

- Physalis pubescens L. var. integrifolia (Dunal) Waterf., Low Hairy Ground Cherry, † Waif Reed (1964)

[Physalis pruinosa L.]

Physalis pubescens L. var. pubescens, Low Hairy Ground Cherry (Wells et al. 4533 US)

[Physalis barbadensis Jacqin; P. floridana Rydb.]

Physalis virginiana Mill. var. virginiana, Virginia Ground Cherry, S3/G5 (House 983 US)

[Physalis intermedia Rydb.; P. monticola C. Mohr]

- Solanum adscendens Sendtn., Sonotia Nightshade, † Waif Reed (1964)
- Solanum americanum Mill., American Black Nightshade-see Excluded Flora section.

[Solanum nodiflorum Jacq.; S. ptychanthum Dunal]

Solanum carolinense L., Carolina Horse Nettle (Dorr 7961 US)

- Solanum deflexum Greenm., Deflexed Nightshade, † Waif Reed (1964)

Solanum dulcamara L., Climbing Nightshade, † (Ubler s.n. US)

- Solanum elaeagnifolium Cav., Silverleaf Nightshade, $†$ Waif Reed (1964)

Solanum emulans Raf., West Indian Nightshade (Knapp 2291 DOV)

Solanum lycopersicum L., Garden Tomato, † (Hill 16002 Assateague Island National Seashore Herbarium)

[Lycopersicon esculentum Mill.]

Solanum nigrum L., Black Nightshade, † (Beyersdorfer 181 US) [Solanum rubrum Mill.]

Solanum physalifolium Rusby, Ground Cherry Nightshade, † (Davis 7823 National Park Service Herbarium at Harpers Ferry)

- Solanum rostratum Dunal, Buffalo Bur, † Waif Reed (1964) [Androcera rostrata (Dunal) Rydb.]

- Solanum sarrachoides Sendtn., Hairy Nightshade-see Excluded Flora section.

- Solanum torvum Sw., Turkey Berry, † Waif Reed (1964)

Solanum tuberosum L., White Potato, † (Choe 008 NA)

- Solanum villosum L., Villose Nightshade, † Waif Reed (1964) [Solanum nigrum L. var. villosum L.]

Solanum virginianum L., Surattense Nightshade, † (Reed 44349 US)

[Solanum surattense Burm. f.]

STAPHYLEACEAE

(Bladdernut Family)

Staphylea trifolia L., American Bladdernut (Naczi 9325 DOV)

STYRACACEAE

(Storax Family)

Halesia carolina L., Carolina Silverbell, † (Morgan 12 DOV)

Halesia tetraptera J. Ellis, Mountain Silverbell, † (Longbottom 7171 DOV)

SYMPLOCACEAE

(Sweetleaf Family)

Symplocos tinctoria (L.) L'Hér., Horsesugar, S3/G5 (Knapp 2375 DOV)

TALINACEAE

(Fameflower Family)

Phemeranthus teretifolius (Pursh) Raf., Roundleaf Fameflower, S2/G4 (Fosberg 18458 US)

[Talinum teretifolium Pursh]

TAMARICACEAE

(Tamarisk Family)

Tamarix parviflora DC., Smallflower Tamarisk, † (Fabula 12 MARY)

- Tamarix ramosissima Ledeb., Saltcedar, † Waif (Bunting 11 MARY) 


\section{TETRACHONDRACEAE}

(Tetrachondra Family)

Polypremum procumbens L., Juniper Leaf (Steury 050820.1 US)

THYMELAEACEAE

(Mezereum Family)

Dirca palustris L., Eastern Leatherwood, S2/G4 (Knapp 2721 DOV)

TOFIELDIACEAE

(False Asphodel Family)

- Triantha glutinosa (Michx.) Baker, Sticky Tofieldia-see Excluded Flora section. [Tofieldia glutinosa (Michx.) Pers.]

Triantha racemosa (Walter) Small, Coastal False Asphodel, - SX/G5 (Roller 871 US)

[Tofieldia racemosa (Walter) Britton, Sterns, \& Poggenb.]

TYPHACEAE

(Cattail Family)

Sparganium acaule (Beeby) Rydb., European Bur Reed, S3/ G5 (Stone $13933 \mathrm{PH}$ )

[Sparganium chlorocarpum Rydb.; S. emersum Rehman]

Sparganium americanum Nutt., American Bur Reed (Benedict 450 US)

Sparganium androcladum (Engelm.) Morong, Branched Bur Reed, SU/G4G5 (McAtee 2318 US)

Sparganium eurycarpum Engelm., Broadfruit Bur Reed, S3/G5 (Topping 149 US)

Typha angustifolia L., Narrowleaf Cattail, $\ddagger$ (Hotchkiss 7192 US) Typha domingensis Pers., Southern Cattail, S3/G4G5 (Hotchkiss 7193 US)

Typha latifolia L., Broadleaf Cattail (Killip 41359 US)

ULMACEAE

(Elm Family)

- Ulmus alata Michx., Winged Elm, † Waif (Erickson s.n. MARY)

Ulmus americana L., American Elm (Beyersdorfer 121 US) [Ulmus floridana Chapm.]

- Ulmus glabra Huds., Wynch Elm—see Excluded Flora section.

- Ulmus minor Mill., English Elm—see Excluded Flora section. [Ulmus procera Salisb.]

Ulmus parvifolia Jacq., Chinese Elm, † (Brown s.n. MARY)

Ulmus pumila L., Siberian Elm, † (Harms 7369 MARY)

Ulmus rubra Muhl., Slippery Elm (Coville 913 US) [Ulmus fulva Michx.]

- Ulmus thomasii Sarg., Rock Elm—see Excluded Flora section.

URTICACEAE

(Nettle Family)

Boehmeria cylindrica (L.) Sw., Small-spike False Nettle (Crawford s.n. US)

[Boehmeria drummondiana Wedd.]

- Boehmeria nivea (L.) Gaudich., Ramie-see Excluded Flora section.
Laportea canadensis (L.) Wedd., Canada Wood Nettle (Hitchcock 15715 US)

- Parietaria floridana Nutt., Florida Pellitory—see Excluded Flora section.

- Parietaria judaica L., Spreading Pellitory—see Excluded Flora section.

Parietaria pensylvanica Muhl. ex Willd., Pennsylvania Pellitory (McAvoy 5109 DOV)

Pilea fontana (Lunell) Rydb., Springs Clearweed, S3/G5 (Steury 031004.5 US)

Pilea pumila (L.) A. Gray, Canada Clearweed (Beyersdorfer 248 US)

[Adicea pumila (L.) Raf.]

- Urtica ballotifolia Wedd., Horehound-leaf Nettle, † Waif Reed (1964)

Urtica dioica L., Stinging Nettle, † (McCallum s.n. CM)

Urtica gracilis Aiton, Slender Stinging Nettle (Boone 820801 TAWES)

[Urtica dioica L. ssp. gracilis (Aiton) Selander]

- Urtica urens L., Dwarf Nettle-see Excluded Flora section

VERBENACEAE

(Verbena Family)

- Glandularia bipinnatifida (Nutt.) Nutt. var. bipinnatifida, Dakota Vervain, † Waif Reed (1964)

[Verbena bipinnatifida Nutt.]

Phyla lanceolata (Michx.) Greene, Fogfruit (Beyersdorfer 154 US)

[Lippia lanceolata Michx.]

- Phyla nodiflora (L.) Greene, Common Fogfruit, † Waif Reed (1964)

[Lippia nodiflora (L.) Michx.; Phyla incisa Small]

Verbena bonariensis L., Purple Vervain, † (Stasz Photo Voucher MPA)

Verbena bracteata Lag. \& Rodr., Large-bract Vervain, † (Sollers s.n. US)

[Verbena bracteosa Michx.]

Verbena brasiliensis Vell., Brazilian Vervain, † (Holt \& Ebert 404 DOV)

Verbena hastata L., Blue Vervain (Fleming 2322 US)

- Verbena neomexicana (A. Gray) Small, Hillside Vervain-see Excluded Flora section.

Verbena officinalis L., European Vervain, † (Leonard \& Killip 807 US)

- Verbena scabra Vahl, Sandpaper Vervain, † Waif Reed (1964)

Verbena simplex Lehm., Narrowleaf Vervain (Bartsch s.n. US) [Verbena angustifolia Michx.]

Verbena urticifolia L., White Vervain (Hitchcock 15754 US) 


\section{VIOLACEAE}

(Violet Family)

Cubelium concolor (T. F. Forst.) Raf. ex Britton \& A. Br., Green Violet, S3/G5 (Hitchcock 12677 US)

[Hybanthus concolor (T. F. Forst.) Spreng.]

Viola affinis Leconte, Le Conte's Violet (Brown s.n. PH)

[Viola sororia Willd. var. missouriensis (Greene) L. E. McKinney]

Viola appalachiensis L. K. Henry, Appalachian Blue Violet, - S3/G4 (Davis 3826)

[Viola walteri House var. appalachiensis (L. K. Henry) L. E. McKinney ex S. P. Grund \& B. L. Isaac]

Viola arvensis Murray, Small Wild Pansy, † (Longbottom 8630 DOV)

Viola bicolor Pursh, Field Pansy (Eggleston 4309 CM)

[Viola kitaibeliana Schult.; V. rafinesquei Greene]

Viola blanda Willd., Smooth White Violet (Crawford s.n. PH)

[Viola blanda var. palustriformis A. Gray; V. incognita Brainerd]

Viola brittoniana Pollard, Coast Violet (McAvoy 3345 DOV)

Viola canadensis L., Canada Violet (House 1522 CM)

Viola cucullata Aiton, Marsh Blue Violet (Henry \& Buker $18244 \mathrm{CM})$

Viola esculenta Elliott, Cypress Violet, S2/G4G5 (Knapp む Wilson 3940 TAWES)

Viola hastata Michx., Halberd-leaf Yellow Violet (Stone s.n. PH)

Viola hirsutula Brainerd, Southern Woodland Violet (Tatnall 2924 DOV)

Viola labradorica Schrank, Alpine Violet (Davis 1381 CM)

[Viola conspersa Rchb.]

Viola lanceolata L., Lanceleaf Violet (Longbottom 4511 DOV)

[Viola lanceolata ssp. vittata (Greene) N. Russell; V. vittata Greene]

Viola macloskeyi F. E. Lloyd ssp. pallens (Banks ex Ging.) M. S. Baker, Smooth White Violet (Longbottom 8581 DOV)

[Viola pallens (Banks ex Ging.) Brainerd]

Viola odorata L., Sweet Violet, † (Romeo 22 MARY)

Viola palmata L., Palmate-leaf Violet (House 935 CM)

[Viola subsinuata Greene; V. triloba Schwein.; V. triloba var. dilatata (Elliott) Brainer]

- Viola patrinii DC., China Violet—see Excluded Flora section.

Viola pedata L., Bird's-foot Violet (Knapp 2709 DOV)

Viola primulifolia L., Primrose-leaf Violet (O’Neil 467 DOV)

Viola pubescens Aiton var. pubescens, Downy Yellow Violet (Knapp 2374 DOV)

[Viola eriocarpa (Nutt.) Schwein. var. eriocarpa]

Viola pubescens Aiton var. scabriuscula Torr. \& A. Gray, Smooth Yellow Violet (Eifrig s.n. CM)

[Viola eriocarpa (Nutt.) Schwein. var. leiocarpa Fernald \& Wiegel; V. pensylvanica Michx.; V. pubescens var. eriocarpa Nutt.; V. pubescens var. leiocarpa (Fernald \& Wiegel) B. Bovin; V. scabriuscula Torr. \& A. Gray]
Viola rostrata Pursh, Long-spur Violet, S3/G5 (Eifrig s.n. CM) Viola rotundifolia Michx., Roundleaf Violet (Stone s.n. PH) Viola sagittata Aiton, Arrowleaf Violet (Tatnall 385 DOV) [Viola emarginata (Nutt.) Leconte; V. fimbriatula Sm.; V. sagittata var. ovata (Nutt.) Torr. \& A. Gray]

- Viola septemloba Leconte, Southern Coastal Violet-see Excluded Flora section.

- Viola septentrionalis Greene, Northern Blue Violet-see Excluded Flora section.

Viola sororia Willd., Woolly Blue Violet (Eifrig s.n. CM) [Viola floridana Brainerd; V. palmata L. var. sororia (Willd.) Pollard; V. papilionacea Pursh; V. priceana Pollard; V. rosacea Brainerd]

Viola striata Aiton, Striped Violet (Eifrig s.n. CM)

Viola stoneana House, Stone's Violet (Long $32210 \mathrm{PH}$ )

Viola tricolor L., Three-colored Violet, † (Longbottom 8363 DOV)

- Viola tripartita Elliott, Three-parted Violet-see Excluded Flora section.

[Viola tripartita var. glaberrima (Ging.) R. M. Harper]

VITACEAE

(Grape Family)

Ampelopsis glandulosa (Wall.) Momiy., Porcelain Berry, $\ddagger$ (Holm s.n. US)

[Ampelopsis brevipedunculata (Maxim.) Trautv.]

- Ampelopsis cordata Michx., False Grape-see Excluded Flora section.

Causonis japonica (Thunb.) Raf., Bushkiller, † (Davis 8021 BALT) [Cayratia japonica (Thunb.) Gagnep.]

- Nekemias arborea (L.) J. Wen \& Boggan, Peppervine-see Excluded Flora section.

[Ampelopsis arborea (L.) Koehne]

- Parthenocissus inserta (A. Kern) Fritsch, Thicket Creeper-see Excluded Flora section.

[Parthenocissus quinquefolia (L.) Planch. var. vitacea Knerr; P. vitacea (Knerr) Hitchc.]

Parthenocissus quinquefolia (L.) Planch., Virginia Creeper (Fosberg 56247 US)

[Parthenocissus hirsuta (Pursh) Grab.]

- Parthenocissus tricuspidata (Siebold \& Zucc.) Planch., Boston Ivy-see Excluded Flora section.

Vitis aestivalis Michx., Summer Grape (Tatnall 1132 DOV) [Vitis aestivalis var. bicolor Deam]

Vitis cinerea Engelm. ex Millard var. floridana Munson, Florida Grape (Leonard 20643 US)

[Vitis simpsonii Munson]

Vitis labrusca L., Fox Grape (Fleming 2307 US)

Vitis riparia Michx., Riverbank Grape (Beyersdorfer 107 US) 
Vitis rotundifolia Michx. var. rotundifolia, Muscadine (McAvoy 2739 DOV)

[Muscadinia rotundifolia (Michx.) Small]

Vitis rupestris Scheele, Sand Grape, S1/G3 (Fisher s.n. US)

Vitis vulpina L., Winter Grape (Fisher s.n. US)

XYRIDACEAE

(Yellow-eyed Grass Family)

- Xyris caroliniana Walter, Carolina Yellow-eyed Grass-see Excluded Flora section.

Xyris difformis Chapm. var. difformis, Bog Yellow-eyed Grass (Strong et al. 2019 US)

Xyris fimbriata Elliott, Fringed Yellow-eyed Grass, S1/G5 (Hirst \& Wilson 141a DOV)

Xyris jupicai Rich., Richard's Yellow-eyed Grass, † (Strong et al. 1756 US)
Xyris smalliana Nash, Small's Yellow-eyed Grass, S1/G5 (Carter s.n. PH)

Xyris torta Sm., Slender Yellow-eyed Grass (Knapp 592 DOV)

ZOSTERACEAE

(Eelgrass Family)

Zostera marina L. var. stenophylla Asch. \& Grab., Eelgrass (Truitt s.n. US)

ZYGOPHYLLACEAE (Creosote-Bush Family)

- Kallstroemia parviflora Norton, Texas Caltrop, † Waif Reed (1964)

Tribulus terrestris L., Puncture-Weed, $¥$ (Longbottom 10468 \& Van Velsir DOV) 



\section{Excluded Flora}

\section{LYCOPHYTES}

\section{ISOETACEAE}

(Quillwort Family)

\section{Isoetes tuckermanii A. Braun}

Attributed to Maryland by Kott and Britton (1982), and all other attributions to the state are seemingly based upon this report. The Flora of North America treatment (Taylor et al. 1993), partially authored by Britton, does not include Maryland in the range. We suspect the early report of Kott and Britton (1982) to have been made in error and have found no voucher to support its inclusion.

\section{FERNS}

\section{CYSTOPTERIDACEAE}

(Bladder Fern Family)

Gymnocarpium appalachianum K. M. Pryer \& Haufler

Pryer's (1993) Flora of North America (FNA) treatment attributed G. appalachianum but not G. dryopteris to Maryland. Gymnocarpium appalachianum was not reported to Maryland by Pryer's previous works (Pryer and Haufler 1993). The Biota of North America Program (Kartesz 2015a,b) attributed G. appalachianum stating "1993. Systematic Botany Monographs"; however, there is no such source. Presumably, this is meant to cite Pryer and Haufler's (1993) paper in Systematic Botany, which does not attribute this species to Maryland. No specimen is known validating Pryer's attribution.

\section{OPHIOGLOSSACEAE}

(Adder's-tongue Family)

Sceptridium jenmanii (Underw.) Lyon

[Botrychium jenmanii Underw.]

Kartesz (2015a,b) and the U.S. Department of Agriculture (2018) PLANTS Database (hereafter USDA PLANTS 2018) cited Wagner and Wagner (1993), but Wagner and Wagner did not attribute Maryland to the range of this more southern species.

\section{PTERIDACEAE (Maidenhair Fern Family)}

Adiantum aleuticum (Rupr.) C. A. Paris

Attributed by various sources indicating this plant is located on serpentine habitats in Maryland and Pennsylvania. Recent molecular work by Williams et al. (2016) identifies Maryland specimens as Adiantum pedatum.
SALVINIACEAE

(Floating Fern Family)

Salvinia minima Baker

[Salvinia rotundifolia auct. non Willd.]

Attributed here by USDA PLANTS (2018) citing Brown and Brown (1984). Brown and Brown (1984:41) stated, "May be found as an escape," which is ambiguous. No specimen can be found confirming its establishment here.

\section{GYMNOSPERMS}

PINACEAE

(Pine Family)

Picea abies (L.) H. Karst

Kartesz (2015a,b) cited the NCU herbarium, yet the only specimen from Maryland at NCU (Downs 7394) clearly states "probably planted" on label. There is no evidence this species is established.

Picea glauca (Moench) Vos

[P. glauca var. densata Bailey]

USDA PLANTS (2018) cited Brown and Brown (1972:10), yet the Browns stated only that it "may be encountered in many plantations" (i.e., directly where it is planted). It is not known outside of cultivation.

Picea pungens Engelm.

Kartesz (2015a,b) and USDA PLANTS (2018) cited Brown and Brown (1972), but the Browns stated only that this species is used as an ornamental. It is not known outside of cultivation.

\section{FLOWERING PLANTS}

ADOXACEAE

(Moschatel Family)

Viburnum molle Michx.

Attributed by Norton and Brown (1946), but no specimen can be found to validate this attribution.

\section{Viburnum rufidulum Raf.}

Attributed by Kartesz (2015a,b) on the basis of the specimen at MARY. The only MARY specimen labeled as V. rufidulum was incorrectly identified and is $V$. prunifolium. 


\section{ALISMATACEAE}

Alisma triviale Pursh

Brown and Brown (1984:55) stated it is "common throughout," yet Maryland is not in the known range of A. triviale.

Helanthium tenellum (Mart. ex Schult. f.) Britton

[Echinodorus tenellus (Mart. ex Schult. f.) Buchenau]

Brown and Brown (1984:55) stated, "Reported from Kent County," citing no source for the attribution. The only location in the area for this species is Kent County, Delaware (Tatnall 1946). This is likely the source of this misattribution.

\section{Sagittaria brevirostra Mack. \& Bush}

Known from adjacent Virginia, this species is known only from literature sources seemingly stemming from Brown and Brown (1984:62), who stated "shallow water; ponds and swamps. Calvert Co.” We have seen no voucher of this species for Maryland.

Sagittaria filiformis J. G. Sm.

[Sagittaria stagnorum Small; S. subulata (L.) Buchenau var. gracillima J. G. Sm.]

All attribution seemingly stems from Brown and Brown (1984:59) stating “may be encountered along the coast." No specimen is known to support its presence here.

\section{Sagittaria teres S. Watson}

Brown and Brown (1984:59) stated, "Shallow water and swamps. Coastal; rare," but no specimen exists to validate this attribution.

\section{AMARANTHACEAE}

(Amaranth Family)

\section{Chenopodium rubrum $\mathrm{L}$.}

[Oxybasis rubra (L.) S. Fuenes-B., Uotila, \& Borsch]

Attributed by Stieber (1971:284) and Brown and Brown (1984:420). Stieber stated, "In saltmarshes, US." Searches at herbaria have found no specimen. We suspect that if a specimen existed, it has been annotated to a different species or lost. Brown and Brown gave no distributional data other than "salt marshes and brackish or saline soils." The distribution data appear to be taken directly from Stieber.

\section{Chenopodium urbicum $\mathrm{L}$.}

[Oxybasis urbica (L.) S. Fuenes-B., Uotila, \& Borsch]

Attributed by Brown and Brown (1984:417), stating "waste areas; occasional." No specimen is known validating this attribution.
AMARYLLIDACEAE

(Amaryllis Family)

\section{Allium allegheniense Small}

Attributed by Kartesz (2015a,b), citing a specimen at NCU. The only specimen at NCU (McFarland s.n.) has been annotated by Weakley as $A$. canadense var. canadense.

\section{Allium sativum $\mathrm{L}$.}

Attribution stems from Stieber (1971), but no specimen has been found validating this report.

\section{ANACARDIACEAE}

(Sumac/Cashew Family)

Cotinus coggygria Scop.

A single specimen (s. coll., s.n. MARY) states only "College Park" and is presumably a collection of a planted individual. There is no evidence that this plant is established outside of cultivation in Maryland.

Toxicodendron rydbergii (Small ex Rydb.) Green

Attributed by numerous sources, yet no specimen is known validating these attributions. If present, it is restricted to western Maryland and likely rare.

APIACEAE [Umbelliferae]

(Carrot/Parsley Family)

Falcaria vulgaris Bernh.

[Falcaria sioides Asch.]

Attributed by Brown and Brown (1984:707), stating “occasional as a weed of fields," but no specimen is known validating this claim.

Oenanthe aquatica (L.) Poir.

Reported in Maryland by Kartesz (2015a,b), saying specimen at US. The only specimen at the US herbarium is from Washington D.C., where it is considered a waif (Shetler and Orli 2000).

Petroselinum crispum (Mill.) Nyman ex A. W. Hill

Attributed to Maryland by various sources, but all specimens seen have been from cultivation.

\section{Scandix pecten-veneris L.}

Shetler and Orli (2000) attributed as a waif, citing McAtee (1930). No specimen has been found to validate McAtee's attribution.

Spermolepis inermis (Nutt. ex DC.) Mathias \& Constance [Spermolepis patens (Nutt. ex DC.) B. L. Rob.]

Attributed by Brown and Brown (1984:717), stating "dry soils. Baltimore County." No specimen can be found validating this attribution. 


\section{APOCYNACEAE}

(Dogbane Family)

Amsonia tabernaemontana Walter var. tabernaemontana

[Amsonia amsonia (L.) Britton]

Attributed by Brown and Brown (1984:744), citing "Rich, moist woods. Coastal." No specimen can be found validating this attribution.

Asclepias linearis Scheele

Attribution of this species stems from a Shreve specimen labeled such, but the specimen is clearly A. verticillata.

Asclepias longifolia Michx.

[Acerates floridana (Lam.) Hitchc.; A. longifolia (Michx.) Elliott]

Reported by Rosatti (1989:497) as "distributed along the coastal plain from Louisiana to Maryland." This generalized range is not supported by any known specimen. The northern limit of this species is a single historic site in Sussex County, Delaware.

Matelea decipiens (Alexander ex Small) Woodson

Attributed by various literature sources. No specimen is known, and this would be the species' northern limit.

\section{ARACEAE}

(Arum Family)

Wolffia borealis (Engelm.) Landolt \& Wildi

[Wolffia punctata auct. non Griseb.]

The initial source of this species attribution to Maryland appears to be Fernald (1950:387) stating "n., very locally, to e. Md." No other monograph or later treatment places this in Maryland. No specimen is known to support this attribution.

\section{ASPARAGACEAE}

(Asparagus Family)

\section{Agave virginica $\mathrm{L}$.}

[Manfreda tigrina (Engelm.) Small; M. virginica (L.) Rose; Polianthes virginica (L.) Shinners]

Reported by Brown and Brown (1984), but no specimen can be found to substantiate this report.

Hosta lancifolia (Thunb.) Engl.

Attributed by Brown and Brown (1984:335), stating "cultivated and escaping." Though likely a rare escape, no specimens are known validating this report.

\section{Liriope graminifolia (L.) Baker}

Reported by Terrell et al. (2000), but the only specimen found is a misidentification of L. spicata.

Liriope muscari (Decne.) L. H. Bailey

USDA PLANTS (2018) and Kartesz (2015a,b) cited J. Reveal (personal communication). Unfortunately, Reveal died in 2016, and no specimen can be found validating this claim.
ASTERACEAE [Compositae]

(Aster Family)

Achillea millefolium L.

[Achillea aspleniifolia Vent.; A. millefolium var. occidentalis

DC.; A. occidentalis (DC.) Raf. ex Rydb.]

Previously thought to be widespread and non-native, this taxon is apparently absent from the flora area. The Achillea found here is A. borealis, a native species.

\section{Artemisia biennis Willd.}

Likely to be found here and reported by various sources as a component of the flora. No specimen can be found validating this attribution.

\section{Artemisia pontica L.}

Attributed by various sources and likely a rare escape. No specimen is known validating this attribution. If this is sporadically encountered, it is likely not truly established.

Bidens beckii (Torr. ex Spreng.) Greene

[Megalodonta beckii (Torr. ex Spreng.) Greene]

Attributed by Brown and Brown (1984:1016), stating "Coastal; rare." This is a northern species ranging as far south as central Pennsylvania and southern New Jersey.

\section{Bidens mitis (Michx.) Sherff}

Variously attributed to the state by numerous sources. All specimens seen have been $B$. trichosperma. This species could be found in the state, but no specimen validating its attribution is currently known.

\section{Centaurea cineraria L.}

Attributed by Brown and Brown (1984:1047) as an "occasional as an escape. Prince George's County," but no specimen in known to validate this attribution.

\section{Centaurea scabiosa L.}

Attributed by Brown and Brown (1984:1047), stating "White Church, Garrett County, and Washington County"; however, no specimen can be found validating this attribution.

\section{Doellingeria sericocarpoides Small}

[Aster sericocarpoides (Small) K. Schum.]

Attributed by Semple and Chmielewski (2006) but all specimens seen (Shriver $238 \mathrm{NY}$ and Blanchard s.n. NY) are D. infirma.

\section{Echinops sphaerocephalus L.}

Attributed by Brown and Brown (1984:1036), stating "Introduced into cultivation from Europe; often escaping." Though this could be the case, there is no specimen validating this claim. If encountered, this species may best be treated as a waif. 


\section{Eupatorium semiserratum DC.}

This species does not range north of southeastern Virginia. Broader attribution is based upon misidentification and unvouchered literature citations.

Glebionis segetum (L.) Fourr.

[Chrysanthemum segetum L.]

Attributed by USDA PLANTS (2018) citing Brown and Brown (1984:1025), but Brown and Brown stated there is "No Maryland record.”

Helianthus microcephalus Torr. \& A. Gray

All known material identified as $H$. microcephalus has been redetermined to $H$. divaricatus.

\section{Helianthus salicifolius A. Dietr.}

Attributed by Brown and Brown (1984:1001), stating "calcareous fields . . . Rare." No specimen is known validating this southwestern species. If it ever occurred here, it was likely a waif from the West.

\section{Helminthotheca echioides (L.) Holub}

[Picris echioides L.]

Attributed by Brown and Brown (1984), but no specimen validates report. If previously present, perhaps only as a waif.

\section{Krigia biflora (Walter) S. F. Blake var. biflora}

[Adopogon virginicum (L.) Kuntze; Cynthia virginica (L.) D. Don]

Attributed by Brown and Brown (1984), yet no known specimen exists validating this report.

\section{Marshallia grandiflora Beadle \& F. E. Boynton}

Broome et al. (1979) reported this from Garrett County, along the Youghiogheny River. The report has not been verified, and no populations have been documented during extensive survey work along the Youghiogheny River. Marshallia grandiflora is best treated as an extinct endemic of North Carolina, with plants in the mid-Atlantic being treated as M. pulchra W. M. Knapp, D. B. Poind, \& Weakley, which is not known from Maryland (Knapp et al. 2020).

\section{Packera glabella (Poir.) C. Jeffrey}

[Senecio glabellus Poir.]

Reported by Brown as Brown (1984:1033) as "Rare. Prince George's County." No specimen can be found for this more southern species.

\section{Parthenium auriculatum Britton}

[P. hispidum Raf. var. auriculatum (Britton) Rollins]

Attributed by Brown and Brown (1984:994), stating "dry shale and calcareous outcrops." This species is known to range only as far north as northeast West Virginia.
Senecio bicolor (Willd.) Viv.

[Senecio cineraria DC.]

First attributed by Brown and Brown (1984:1033), stating "cultivated and rarely found as an escape; College Park, Prince George's County." No specimen can be found supporting this attribution.

Silphium perfoliatum L. var. connatum Cronquist

Brown and Brown (1984:993) stated, "In our range according to Harned [(1936)]; Baltimore County (Baltars). Most of our plants are var. connatum." The modern understanding of this variety is that it is endemic to the New River Valley of North Carolina, Virginia, and West Virginia. No specimen has been found validating this attribution, and Cylburn Arboretum Herbarium, home to many Baltars collections, was inaccessible to us.

\section{Silphium perfoliatum L. var. perfoliatum}

Brown and Brown (1984:993) stated, "In our range according to Harned [(1936)]; Baltimore County (Baltars). Most of our plants are var. connatum." This infers some are var. perfoliatum. If this were found in Maryland, it was likely as a garden escape. No specimen has been found validating this attribution, and Cylburn Arboretum Herbarium, home to many Baltars collections, was inaccessible to us.

Solidago arguta Aiton var. boottii (Hook.) E. J. Palmer \& Steyerm.

Attributed by Brown and Brown (1984), but in the East, this variety ranges only as far north as central South Carolina. Apparently, Brown and Brown included it because they had a different taxonomic concept than the one currently used.

\section{Solidago curtisii Torr. \& A. Gray}

[Solidago lancifolia Torr. \& A. Gray; S. pubens M. A. Curtis]

Reported in Maryland by Cusick (1986), but all specimens cited have been annotated by specialist John Semple as other Solidago species.

\section{Solidago missouriensis Nutt.}

Attributed by Brown and Brown (1984:956), stating "has been reported from Howard and Baltimore Counties, introduced from west." We have found no specimen validating this attribution.

Solidago puberula Nutt. var. pulverulenta (Nutt.) Chapm. [Solidago pulverulenta Nutt.]

Attributed by Brown and Brown (1984), but this is most likely based upon misattribution. In current usage, this variety is believed to reach its northern limit in southeastern Virginia.

Solidago rugosa Mill. var. sphagnophila G. Graves

Attributed by Weakley (2015), but lacking evidence to support this attribution. Attribution will be removed from future 
editions of the flora (A. Weakley, University of North CarolinaChapel Hill, personal communication).

\section{Solidago stricta Aiton}

Attributed by Brown and Brown (1984), but no specimen exists validating this claim. Specimens identified as $S$. stricta have been annotated as $S$. sempervirens var. mexicana.

Tripleurospermum inodorum (L.) Schultz

[Matricaria inodora L.; Tripleurospermum perforatum (Mérat) M. Lainz]

Attributed by Brouillet (2006), but no specimen is known to support this attribution.

\section{Verbesina virginica $\mathrm{L}$.}

Attributed by Brown and Brown (1984:1007), stating “Dry woods and clearings. Infrequent.” No specimen has been found validating this attribution.

\section{BERBERIDACEAE}

(Barberry Family)

\section{Berberis canadensis Mill.}

Attributed by numerous sources, all based on the literature or incorrectly identified specimens. All specimens we have seen that had been identified as $B$. canadensis are B. thunbergii.

\section{BETULACEAE}

(Birch Family)

\section{Betula papyrifera Marsh}

Brown and Brown (1972) stated this species "has been reported for Maryland," but no specimens outside of cultivation have been found.

\section{BORAGINACEAE}

(Borage Family)

\section{Asperugo procumbens L.}

Listed as a waif by Hermann (1941), but the only specimens known are from Washington, D.C.

\section{Borago officinalis L.}

Listed as a waif by Hermann (1946) with no additional information. The only known specimens found are from Washington D.C.

Lithospermum molle (Michx.) Muhl.

[Onosmodium molle Michx. var. hispidissimum (Mack.) Cronquist]

Attributed by the Maryland Natural Heritage Program (2010). This attribution was based upon confusion with Lithospermum parviflorum.

\section{Phacelia bipinnatifida Michx.}

[Phacelia brevistyla Buckley]

Attributed by Kartesz (2015a,b), citing "Radford et al. (unpublished)." There is no way to validate this unpublished citation, and no specimen has been found validating this attribution.

Phacelia ranunculacea (Nutt.) Constance

Reported by Brown and Brown (1984), but this name applies to a species from the central United States and not our Maryland material. Our plants are P. covillei.

Symphytum asperum Lepech.

Reported by Fernald (1950) as occurring as far south as Maryland and by Brown and Brown (1984:777) as a "Rare escape. From Europe.” No specimen is known validating these reports.

BRASSICACEAE [Cruciferae]

(Mustard Family)

Cardamine flexuosa With.

All plants attributed to C. flexuosa are C. occulta.

Cardamine pratensis L. var. palustris Wimm. \& Grab.

All specimens seen have been identified as various other taxa in Cardamine.

Erysimum asperum (Nutt.) DC.

[Cheirina aspera (Nutt.) Britton]

Name has been misapplied to E. capitatum.

Erysimum inconspicuum (S. Watson) MacMill.

Attributed to Maryland by Kartesz (2015a,b), citing a specimen at MARY, and Brown and Brown (1984). The specimen at MARY says "Washington" as the locality. This species could be expected to occur in Maryland but is here excluded because evidence is lacking it is established. Brown and Brown (1984:514) stated, "Dry soils of fields and wooded areas," but no specimen can be found to validate this claim.

BUXACEAE

(Boxwood Family)

Pachysandra procumbens Michx.

Attributed by Kartesz (2015a,b), citing a specimen from Baltimore County. The specimen, Pelletier 20 MARY, is P. terminalis.

\section{CAMPANULACEAE}

(Bellflower Family)

Campanula divaricata Michx., SH/G4

Though attributed by Brown and Brown (1984) and appearing on the state's list of rare, threatened, and endangered species (Maryland Natural Heritage Program 2018), there is no specimen or photograph validating this as a component of the flora.

\section{Jasione montana L.}

Attributed by USDA PLANTS (2018), citing Hultén and Fries (1986). The range map provided for this species contains a dot that looks centered in southeastern Pennsylvania or southwestern New Jersey. If this map does reflect Maryland, there is no specimen known validating its occurrence here. 


\section{Lobelia dortmanna L.}

Attributed by Hultén and Fries (1986). This is an error created by a mapping issue with the revision of Hultén (1958). The maps in Hultén and Fries (1986) are slightly shifted southward relative to Hultén (1958), making a dot that appeared in Pennsylvania in the 1958 edition appear in Maryland in the 1986 edition.

\section{Lobelia glandulosa Walter}

Attributed by Moldenke (1942), citing a specimen at NY. We could not find a voucher at NY.

\section{CANNABACEAE}

(Hemp Family)

\section{Celtis laevigata Willd.}

[Celtis mississippiensis Bosc]

Attributed to Maryland by many literature sources and could occur here, as it is known from northern Virginia. No specimen is known to support its inclusion, and specimens originally identified as Celtis laevigata have been annotated to either C. occidentalis or C. tenuiflora.

\section{CAPRIFOLIACEAE}

(Honeysuckle Family)

Knautia arvensis (L.) J. M. Coult.

Attribution stems from Brown and Brown (1984:911), stating "fields \& waste areas. Native of Europe and now becoming naturalized." No specimen is known validating this report.

\section{Linnaea borealis $\mathrm{L}$.}

Fernald (1950) attributed it to Maryland, but no other source lists this. It is likely the specimen with a handwritten label saying "Pine Station, Ind" (Hill s.n. NY) could be the source of this misattribution as to the unfamiliar eye the "Ind" on the specimen label appears to be "Md." See similar discussion under Poa languida.

\section{Valeriana officinalis L.}

Attributed by Brown and Brown (1984:906), stating "Introduced from Europe and escaping. Garrett Co." If a specimen is found, this species is perhaps best treated as a waif.

\section{Valerianella chenopodiifolia (Pursh) DC., S1/G5}

Reported by the Maryland Natural Heritage Program (2018) from western Maryland, but no specimen has been found validating this attribution. It is likely to occur here based on the overall biogeographic distribution of the species but is excluded until proof of occurrence can be confirmed. Specimens from the central part of Maryland have been misidentifications.

\section{CARYOPHYLLACEAE}

(Pink Family)

\section{Corrigiola litoralis L.}

All sources of attribution cite Brown and Brown (1984:439), who stated it is "Waste areas and fields. Coastal; rare and local." No specimen can be found validating this attribution.
Dianthus deltoides L.

Attributed by Hermann (1946) as an escape in Montgomery County. No specimen is known validating this report.

Paronychia argyrocoma (Michx.) Nutt.

Attributed by Brown and Brown (1984:439), stating "Rock outcrops. Rare; Croply [sic], Montgomery County.” Cropley has traditionally included portions of Bear Island, one of the few Maryland locations for P. virginiana. Furthermore, the distribution and frequency Brown and Brown (1984) gave for P. virginiana are erroneous, "rocky places and forests, Common throughout." Clearly, there was confusion regarding the identification of these two species.

\section{Silene chalcedonica Krause}

Kartesz (2015a,b) reported from Prince George's County referencing a specimen at MARY. No specimen can be found validating this report.

\section{Silene conica L.}

Attributed by Brown and Brown (1984:453), stating “Anne Arundel County, and Delaware; infrequent." No specimen is known validating this statement. If previously found here, this species is perhaps best treated as a waif.

\section{Silene conoidea $\mathrm{L}$.}

Attributed by Brown and Brown (1984:453), stating "escaping in coastal areas. Delaware; rare." No specimen is known validating this attribution. If previously found here, this species is perhaps best treated as a waif.

\section{Silene drummondii Hook.}

[Lychnis drummondii (Hook.) S. Watson]

Brown and Brown (1984:449) stated, "From Carroll County ... reported by Baltars." No specimen is known to validate this report.

\section{Silene virginica L.}

Attributed by Luttman (1935) from Garrett County, but searches at multiple herbaria have found no specimen validating this report.

\section{Stellaria borealis Bigelow var. borealis}

Attributed by Morton and Rabeler (1989), which contains a distribution map including Maryland. Searches at herbaria, including WAT, have yielded no specimens from Maryland.

\section{Stellaria neglecta Weihe}

Attributed by Morton (2005). Morton is now deceased, and the source of his attribution is unknown. 


\section{COLCHICACEAE}

(Meadow Saffron Family)

\section{Colchicum autumnale L.}

Attribution stems from Brown and Brown (1984:335), stating "rarely found as an escape." No specimen is known validating this report, and if one exists, this species is perhaps best treated as a waif.

\section{COMMELINACEAE}

(Spiderwort Family)

\section{Cuthbertia graminea Small}

[Callisia graminea (Small) G. C. Tucker; Tradescantia rosea Vent. var. graminea (Small) E. S. Anderson \& Woodson]

Attributed by Brown and Brown (1984:307), stating "Sandy woodland. Infrequent. Our plants belong to the var. graminea." No specimen is known validating this report.

\section{Cuthbertia rosea (Vent.) Small}

[Callisia rosea (Vent.) D. R. Hunt; Tradescantia rosea Vent.]

First attributed to Maryland by Robinson and Fernald (1908). This species is known to range only as far north as North Carolina. No specimen is known validating this report. Fernald (1950) later dropped this attribution to Maryland.

\section{CONVOLVULACEAE}

(Morning-glory Family)

Convolvulus wallichianus Spreng.

Attributed by Witman (1954); however, no specimen can be found to validate this attribution.

\section{Cuscuta cephalanthi Engelm.}

Attributed by Kartesz (2015a,b) from Montgomery County. The label on a specimen at US (Mohr s.n.) from "Little Falls" does not indicate if the specimen was collected in Maryland or Washington, D.C., and therefore, it is excluded but is likely the source of the Montgomery County attribution.

\section{Cuscuta epilinum Weihe}

Attribution of this species to Maryland stems from Hultén and Fries (1986), which contains a mapped dot in eastern Maryland. No specimen is known to support this attribution, and its occurrence here is unlikely.

\section{Cuscuta epithymum (L.) L.}

Brown and Brown (1984:764) stated, "Rare; mostly on clovers ... midland area." No specimen can be found validating this report, and specimens previously identified as C. epithymum have been determined otherwise.

\section{Dichondra carolinensis Michx.}

[Dichondra evolvulacea (L. f.) Britton]

USDA PLANTS (2018) cited Gleason (1963). It is unclear what this citation is referencing, but we expect it is meant to be Gleason and Cronquist (1963). There is no attribution to Maryland in Gleason and Cronquist (1963), and no specimen is known to validate this attribution.

\section{Ipomoea quamoclit L.}

[Ipomoea vulgaris Choisy]

Attributed by Brown and Brown (1984:756), saying "locally persistent after cultivation." No specimen can be found validating this report.

\section{CYPERACEAE}

(Sedge Family)

Bolboschoenus maritimus (L.) Palla ssp. paludosus (A. Nelson) T. Koyama

[Scirpus paludosus A. Nelson]

Attributed by Kartesz (2015a,b), but no specimen is known to validate this attribution. Specimens identified as B. maritimus have been $B$. fluviatilis.

\section{Carex aquatilis Wahlenb.}

All attribution seemingly stems from Hotchkiss \& Ubler 7087 (US), but this specimen is C. emoryi, or Reveal 7587 MARY, which is too immature for proper identification but is certainly not C. aquatilis.

\section{Carex caryophyllea Latourr.}

Attributed by Brown and Brown (1984), USDA PLANTS (2018), and Kartesz (2015a,b), but no specimen is known validating this attribution. All attribution apparently stems from McAtee (1930).

\section{Carex cephaloidea (Dewey) Dewey}

Though mapped from Allegany County by Frye and Lea (2001), the three specimens housed at MARY that are the basis of these attributions are C. aggregata and C. sparaginoides.

\section{Carex divulsa Stokes}

Collected from Washington, D.C., with no collection known from Maryland. Numerous sources erroneously attribute this species to Maryland based on the Washington, D.C., records.

\section{Carex foenea Willd.}

[Carex siccata Dewey]

Attributed by Brown and Brown (1984:249), stating "Dry soil. Midland; infrequent." All attribution appears to stem from this source. No specimen has been found backing this attribution.

\section{Carex novae-angliae Schwein.}

Attributed by Brown and Brown (1984:267), stating “moist woods." No specimen is known to support this attribution.

\section{Carex pallescens L.}

Known from Washington, D.C., with no known specimen supporting its attribution to Maryland. Attributed by USDA PLANTS (2018), citing Frye and Lea (2001), but Frye and Lea do not attribute this to Maryland. 


\section{Carex retrorsa Schwein.}

Attributed by Brown and Brown (1984:292), stating "wet woods and fields; rare." No specimen is known to validate this attribution.

\section{Carex rostrata Stokes}

Attributed by Brown and Brown (1984:292), citing "In the W-B [Washington-Baltimore] area; uncommon." yet no specimen is known validating this report. This attribution is likely based on nomenclatural confusion and a species concept issue involving C. utriculata.

\section{Carex sartwellii Dewey}

Attribution stems from Frye and Lea (2001) from Garrett County. This attribution is based upon a single specimen housed at MARY. The specimen initially identified as C. sartwellii (Norton 1911) is Carex divisa. Another sheet housed at MARY (Engh s.n.) is a very immature member of section Acrocystis.

\section{Carex tenuiflora Wahlenb.}

Attributed by Brown and Brown (1984:256), citing "Bogs and swampy woods," yet no specimen is known validating this report.

\section{Carex verrucosa Muhl.}

Attributed by Norton and Brown (1946), but this southern species ranges only as far north as North Carolina. Likely attributed on the basis of confusion with C. glaucescens.

\section{Carex viridula Michx.}

Attributed by USDA PLANTS (2018), citing Frye and Lea (2001), yet Frye and Lea attribute this species only to Washington, D.C.

\section{Cyperus engelmannii Steud}

Attributed to Maryland from many literature sources from Fernald (1950) onward. No specimen has been found to validate this attribution.

\section{Eleocharis fallax Weath.}

Attributed to Maryland on the basis of nomenclatural confusion. See Smith et al. (2002) for clarification.

\section{Eleocharis flavescens (Poir.) Urb.}

Attributed to the flora by various sources. Attribution is based upon confusion with E. olivacea.

Eleocharis geniculata (L.) Roem. \& Schult.

[Eleocharis capitata (L.) R. Br. var. dispar (E. J. Hill) Fernald]

Attributed to Maryland by Brown and Brown (1984), yet no specimen is known to validate this claim. Its occurrence in Maryland is unlikely from a biogeographic perspective.

\section{Eleocharis ovata Torr.}

Attribution is based upon confusion with E. obtusa.

Fimbristylis puberula (Michx.) Vahl var. puberula, SU/G5 [Fimbristylis spadicea (L.) Vahl]

All attribution stems from Norton and Brown (1946). Its inclusion was likely based upon confusion with $F$. castanea. No specimen is known validating this attribution.

\section{Rhynchospora capillacea Torr.}

Reported by Kartesz (2015a,b), citing a collection at US. No collection was found there to confirm this attribution.

\section{Rhynchospora filifolia A. Gray}

A known component of the Delaware flora, all attributions to Maryland are based on confusion with $R$. harperi.

Schoenoplectus acutus (Muhl. ex Bigelow) Á. Löve \& D. Löve var. acutus

Attributed by Smith (2002), but specimens supporting this attribution are not known to exist (G. Smith, University of Wisconsin, personal communication). A specimen from Arlington, Virginia, is at US, and its close proximity to Maryland may be the source of this attribution. All other attribution is based upon misidentifications of $S$. tabernaemontani.

\section{Scirpus lineatus Michx.}

Attributed by Brown and Brown (1984:225), stating "common in Western Maryland, particularly in Garrett County.” No specimen is known to validate this statement, and no other treatment of the genus or database includes Maryland in the range. Attribution is likely based on confusion with $S$. pendulus.

\section{ELAEAGNACEAE}

(Oleaster Family)

Elaeagnus multiflora Thunb.

First attributed by McAtee (1930), and modern attribution stems from this unvouchered report. Old specimens at MARY are likely from cultivation with no evidence of persistence or escape.

\section{ERICACEAE}

(Heath Family)

Rhododendron calendulaceum (Michx.) Torr.

[Azalea calendulacea Michx.]

Attributed by Brown and Brown (197:251), stating "known only from Garrett County" and listed as SR (state reported) by the Maryland Natural Heritage Program (2018). No specimen can be found to validate this distribution. Other attribution is likely based on color forms of R. periclymenoides or cultivated plants.

\section{Rhododendron canescens (Michx.) Sweet}

All specimens seen attributed as such are pubescent forms of $R$. periclymenoides. See Judd and Kron (2009) for discussion of the characters and true range of $R$. canescens. 


\section{EUPHORBIACEAE}

Croton michauxii G. L. Webster

[Crotonopsis linearis Michx.]

All specimens identified as this species have been Croton willdenowii.

Euphorbia lucida Waldst. \& Kit.

Attributed by Witman (1954), but no specimen is known validating this report.

Euphorbia pubentissima Michx.

[Euphorbia corollata L. var. zinniiflora (Small) H. E. Ahles; Euphorbia zinniiflora Small]

Though attributed by Park (1998), who cited Windler \& Lombardo 3740 (GA), this specimen is in actuality E. corollata. All Maryland material identified as E. pubentissima has been misidentified E. corollata.

FABACEAE [Leguminosae]

(Pea/Bean Family)

Apios priceana B. L. Rob.

Brown and Brown (1984) attributed Baltars as reporting this in Harford County. Given the known geographic range of this species, this unvouchered report is dubious.

Crotalaria rotundifolia (Walter) Poir.

[Crotalaria angulata Mill.; C. maritima Chapm.]

Reported by C. Ludwig from Worcester County (C. Ludwig, Maryland Natural Heritage Program, personal communication). No specimen is known to support this report. Brown et al. (1987:259) cited a specimen, Sherard s.n. OXF, but also state "it is possible it was collected in Virginia.”

Desmanthus illinoensis (Michx.) MacMill.

Kartesz (2015a,b) placed it in Maryland, but the only specimens seen are from Washington, D.C. If this species was found in Maryland, we expect it was not established.

\section{Desmodium tenuifolium Torr. \& A. Gray}

Attributed by Isely (1990), but no specimen can be found validating this attribution.

Galactia volubilis (L.) Britton var. volubilis, S3/G5

[Galactia macreei M. A. Curtis]

Attribution based upon confusion with G. brachypoda. Galactia volubilis var. volubilis is known only as far north as southeastern Virginia.

\section{Genista tinctoria L.}

Reported by Kartesz (2015a,b), but the only specimens seen are from Washington, D.C.
Lablab purpureus (L.) Sweet

[Dolichos lablab L.]

Brown and Brown (1984:593) stated, "Cultivated and occasionally escaping, D.C. area and Baltimore County." No specimen is known to validate this claim. If it were ever found in Maryland, it was likely a waif.

Senna occidentalis (L.) Link

[Cassia occidentalis L.]

Attributed by USDA PLANTS (2018), citing Isely (1990:39). However, Isely does not attribute Maryland but does say "Ala, Ark, Fla, Ga, La, Miss, and Sc (waif records from further north).”

Vicia americana Muhl. ex Willd. var. americana, SH/G5

Attribution is based on Longbottom et al. 4104 (Chris Frye, Maryland Natural Heritage Program, personal communication), but the identification cannot be evaluated since the specimen cannot be located.

FAGACEAE

(Beech Family)

\section{Fagus sylvatica L.}

USDA PLANTS (2018) attributed Maryland, citing Brown and Brown (1972), but Brown and Brown state only that it is planted as an ornamental.

Quercus laurifolia Michx.

Attributed by USDA PLANTS (2018), citing Brown and Brown (1984). We expect USDA PLANTS meant to cite the 1972 Woody Plants of Maryland not the 1984 Herbaceous Plants. Regardless, there is no mention of this species in Brown and Brown (1972, 1984).

\section{GENTIANACEAE}

(Gentian Family)

Gentiana alba Muhl. ex Nutt.

Kartesz (2015a,b) and USDA PLANTS (2018) attributed it to Maryland, citing "Radford et al. Vascular flora of the southeastern United States, Unpublished and undated." No specimen can be found validating this curious literature source.

\section{Gentiana autumnalis L.}

USDA PLANTS (2018) attributed this species to Maryland, citing Brown and Brown (1984:739). Brown and Brown do not attribute this species to Maryland, and they state, "On the sandy pine barrens of the Coastal Plain; one collection from Delaware.”

\section{GERANIACEAE}

(Geranium Family)

Geranium pratense L.

Attributed by Kartesz (2015a,b) based upon a misidentified specimen of Geranium maculatum (Holmes 024 MARY). 


\section{GROSSULARIACEAE}

(Gooseberry Family)

Ribes hirtellum Michx.

Tracked by the Maryland Natural Heritage Program (2010) but later dropped as a rare species because the only known specimen was from a planted individual (Leonard 20165 US).

\section{Ribes nigrum Michx.}

Attributed by Kartesz (2015a,b) and USDA PLANTS (2018), citing Brown and Brown (1972). Brown and Brown (1972:118) mentioned this taxon only in the introduction to the genus and said, "Some escaped species that may be encountered in Maryland are ... R. nigrum, introduced from Eurasia." We have no evidence that this species has ever been found outside of cultivation in Maryland.

\section{HYPERICACEAE}

(St. John's-wort Family)

Hypericum ascyron L. ssp. pyramidatum (Aiton) N. Robson

All references stem from Kalm's (1772) explorations of North America. Unfortunately, Kalm made no collections, so the basis of this claim cannot be validated.

\section{Hypericum mitchellianum Rydb.}

Reported by Hickey (1975), but specimens determined as such are incorrectly identified (Hickey s.n. BALT).

\section{Hypericum tubulosum Walter}

[Triadenum tubulosum (Walter) Gleason]

All specimens determined as this species are $H$. walteri.

\section{IRIDACEAE}

(Iris Family)

Iris ensata Thunb.

[Iris kaempferi Siebold ex Lam.; Limniris ensata (Thunb.) Rodion.]

Reported by Kartesz (2015a,b) and Brown and Brown (1984:365). Brown and Brown stated, "Frequent at old home sites and along road banks where rhizomes have been discarded." No specimen has been found supporting this statement. If present in Maryland, it is likely a waif of old home sites and not an established component of the flora.

\section{JUNCACEAE}

(Rush Family)

Juncus brachycephalus (Engelm. ex A. Gray) Buchenau, SH/G5

Specimens identified as J. brachycephalus by F. J. Hermann at NA and US are J. subcaudatus.

\section{Juncus filiformis L.}

Brown and Brown (1984:312) stated, "In our range for Garrett County according to Gray's Manual”; however, neither Fernald (1950) nor Robinson and Fernald (1908) attributed this plant to Maryland.
Juncus polycephalos Michx.

[Juncus polycephalus Michx.-—orthographic variant]

First attributed to Maryland by Redmond (1932) and perpetuated in the literature subsequently (e.g., Brooks and Clemants 2000). There is no specimen backing Redmond's report. Other attributions of $J$. polycephalus to the state have been based upon Redmond's literature citation or misidentified specimens of $J$. validus (Knapp 2014).

\section{JUNCAGINACEAE}

(Arrowgrass Family)

\section{Triglochin maritima L.}

Brown and Brown (1984) cited this from coastal Maryland, but no specimen is known to validate this report.

\section{LAMIACEAE [Labiatae]}

(Mint Family)

Clerodendrum japonicum (Thunb.) Sweet

Attributed by USDA PLANTS (2018), citing Gleason and Cronquist (1963), but no attribution to Maryland is found there.

Clinopodium acinos (L.) Kuntze

[Acinos arvensis (Lam.) Dandy; Satureja acinos (L.) Scheele]

Attributed by Brown and Brown (1984:818), stating “an introduced weed; reported from Garrett County (Baltars)." If a specimen exists, it may be at the Cylburn Arboretum Herbarium, which we were unable to access.

\section{Dracocephalum parviflorum Nutt.}

Historic collections are from Washington, D.C., and this species is not known in Maryland. Attributed by Kartesz (2015a,b), citing no specific source.

\section{Hedeoma hispida Pursh}

Brown and Brown (1984:816) stated, "Introduced from farther west," but no specimen can be found to validate this claim. If previously found in Maryland, this species is best treated as a waif.

\section{Physostegia purpurea (Walter) S. F. Blake}

Attributed by Brown and Brown (1984:805), stating "wet habitats on the Coastal Plain; reported from the W-B [Washington-Baltimore] area"; this species ranges only as far north as eastern North Carolina.

Pycnanthemum flexuosum (Walter) Britton, Sterns, \& Poggenb.

Attributed by Brown and Brown (1984:821), stating "Dry soils of fields and woods; also in bogs. Common throughout," yet there are no known specimens validating this species north of southeastern Virginia.

\section{Pycnanthemum pycnanthemoides Fernald, SH/G5}

Though listed as historic/extirpated by the Maryland Natural Heritage Program (2018) and attributed by Brown and 
Brown (1984:824), there is no specimen known to confirm this species' existence in Maryland. Brown and Brown stated, "Mostly in mountain woodlands." This species is often confused with $P$. incanum in our range.

\section{Salvia verticillata $\mathrm{L}$.}

Brown and Brown (1984:812) stated, "Fields and waste areas; adventive from Europe. Infrequent." No specimen is known validating this attribution, but if this species was present, it is likely best treated as a waif.

Scutellaria parvula Michx. var. parvula

[Scutellaria campestris Britton]

Attributed by various sources. No specimen is known validating any of these attributions.

\section{Stachys clingmanii Small}

Attributed by Brown and Brown (1984), citing Baltars. This species is a southern Appalachian endemic known only from southwestern North Carolina and southeastern Tennessee (Weakley 2015).

\section{Stachys cordata Riddell}

[Stachys riddellii House]

Tracked by the Maryland Natural Heritage Program (2010) and attributed by Kartesz (2015a,b) and USDA PLANTS (2018), but all specimens identified as this have been other species. Attribution likely stems from confusion with $S$. eplingii.

\section{Stachys latidens Small ex Britton}

Maryland material attributed to this species was misidentified. This species ranges as far north as southwestern Virginia and southeastern West Virginia.

Stachys nuttallii Shuttlew. ex Benth.

Variously attributed to Maryland on the basis of misidentified specimens. This southern Appalachian endemic would be well out of range in Maryland.

\section{Stachys pilosa Nutt.}

Attributed by Kartesz (2015a,b), but no specimen is known validating this attribution.

Thymus praecox Opiz ssp. arcticus (Durand) Jalas

Attributed by USDA PLANTS (2018), citing Brown and Brown (1984), but no attribution is contained in Brown and Brown. The basis of this error is likely an issue of synonymy with T. serpyllum.

\section{Vitex agnus-castus L.}

All specimens examined are clearly from cultivation.
Vitex negundo L.

[Vitex negundo var. heterophylla (Franch.) Rehder]

All specimens examined are clearly from cultivation.

LILIACEAE

(Lily Family)

Erythronium umbilicatum C. R. Parks \& Hardin

All specimens identified as E. umbilicatum are E. americanum. Morphologically, our plants can approach E. umbilicatum, which is more southern.

\section{Lilium grayi Watson}

Attributed to Maryland by Hickey (1975), but this specimen was a misidentification of Lilium canadensis (Hickey II 354 BALT).

Prosartes maculata (Buckley) Britton

[Disporum maculatum (Buckley) Britton]

Reported by Brown and Brown (1984:345), stating "Rich woods; rare." In the Appalachians, this species ranges north to southern Virginia and West Virginia, and we have seen no vouchers from Maryland.

\section{Streptopus amplexifolius (L.) DC. var. americanus}

First attributed to Maryland by Luttman (1935), which is likely the source for broader attribution (i.e., FNA). No specimen is known validating this report.

\section{LINACEAE}

(Flax Family)

\section{Linum floridanum (Planch.) Trel. var. floridanum}

This is a species ranging from NC southward, which has been variously misattributed to Maryland on the basis of literature sources and incorrectly identified specimens of Linum intercursum.

\section{LOGANIACEAE}

(Logania Family)

\section{Spigelia marilandica L.}

[Spigelia corymbosa Raf.]

Attributed in Comments on the Vegetation of Colonial Maryland by Brown et al. (1987), citing a specimen at BM. This specimen is well outside its natural range, despite its name, which reaches northeastern Tennessee, and likely persisted after cultivation, if it was ever truly established in Maryland.

\section{MALVACEAE}

(Mallow Family)

\section{Althaea officinalis L.}

Included by Brown and Brown (1984:638), stating “occasionally naturalized in marshes of the Coastal Plain; also the Upper Patuxent Estuary," but no specimen can be found to validate this statement. Attributed to Maryland by Hill (2016). S. R. 
Hill (Illinois Natural History Survey, personal communication) later stated that the only Maryland material he has seen came from cultivation.

Firmiana simplex (L.) W. Wright

[Firmiana platanifolia (L. f.) Schott \& Endlicher; Sterculia platanifolia L. f.]

Kartesz (2015a,b) attributed this species to Maryland, citing “1983 SIDA.” Presumably, this is Whetstone's (1983) treatment of the Sterculiaceae. In this treatment, the range map for this species shows Washington, D.C., and not Maryland.

\section{Lavatera trimestris L.}

Attributed by Kartesz (2015a,b), but all specimens known are directly from cultivation.

Malva pusilla $\mathrm{Sm}$.

Kartesz (2015a,b) cited Shetler and Orli (2000). Shetler and Orli (2000) contained no attribution of this species to Maryland.

\section{Malva verticillata $\mathrm{L}$.}

Attributed by Brown and Brown (1984:638), stating "found occasionally an escape of waste places." The only specimens seen have been from Washington, D.C.

\section{MELANTHIACEAE}

(Bunchflower Family)

\section{Xerophyllum asphodeloides (L.) Nutt.}

Attributed to Maryland by Brown and Brown (1984:325) with the ambiguous attribution of "Sandy barrens of the Eastern Shore. Rare; known from Delaware." This species is extirpated in Delaware and most likely attributed to Maryland on the basis of it being known from Delaware. Kartesz (2015a,b) attributed this species to Maryland citing a specimen at MARY. No specimen could be found at MARY validating this claim.

\section{MELASTOMATACEAE}

(Melastome Family)

\section{Rhexia aristosa Britton}

A known rare component of the Delaware flora. Brown and Brown (1984:673) stated, "Rare and local on the Eastern Shore." All attributions to Maryland are erroneous literature sources. Specimens identified as R. aristosa have been R. virginiana.

\section{Rhexia nashii Small}

Attributed by Kartesz (2015a,b), citing Kral and Bostick (1969). Though Kral and Bostick map a location for Maryland, there is no reference to this in their text. We strongly suspect the mapped dot for Maryland to be in error.

\section{Rhexia petiolata Walter}

Attributed by Brown and Brown (1984), but no specimen is known supporting this more southern species.
MELIACEAE

(Mahogany Family)

Toona ciliata Roem.

USDA PLANTS (2018) reported this species from Maryland on the basis of "Melvin Brown pers. comm." No voucher is known to exist to validate this report.

NELUMBONACEAE

(Lotus Lily Family)

Nelumbo nucifera Gaertn.

Attributed to Maryland by the Flora of North America (Wiersema 1997). This attribution is erroneous and based upon a specimen from Washington, D.C. (J. Wiersema, USDA, personal communication).

\section{NYMPHAEACEAE}

(Water Lily Family)

Nymphaea odorata Aiton ssp. tuberosa (Paine) Wiersema \& Hellquist

[Nymphaea tuberosa Paine]

Attributed by Fernald (1950), but no specimen can be found to validate this attribution. In the Northeast this plant is more northern, ranging as far south as Pennsylvania (Wiersema 1997).

\section{OLEACEAE}

(Olive Family)

Jasminum nudiflorum Lindl.

USDA PLANTS (2018) attributed this to Maryland, citing Brown and Brown (1972). Though Brown and Brown included this species, they discuss only its affinities to Forsythia.

\section{ONAGRACEAE}

(Evening-primrose Family)

Epilobium strictum Muhl. ex Spreng., S1/G5

First reported by Tatnall (1946), citing an Otis specimen (s.n. DOV), but that specimen is E. leptophyllum. Bartgis reported it from Mount Nebo bog (C. Frye, Maryland Natural Heritage Program, personal communication), a site known for supporting many species of northern affinities, but no specimen can be found validating this attribution.

\section{Ludwigia brevipes (Long) Eames}

Attributed by Kartesz (2015a,b) and the Maryland Natural Heritage Program (2010), but the only known specimen previously identified as this plant (Davis 4426 a TAWES) is Lindernia dubia.

\section{ORCHIDACEAE}

(Orchid Family)

Platanthera integra (Nutt.) A. Gray

[Gymnadeniopsis integra (Nutt.) Rydb.; Habenaria integra (Nutt.) Spreng.]

Luer (1975) mapped Maryland in its range. No specimen is known validating this attribution. We believe this map to be in error. 
Platanthera macrophylla (Goldie) P. M. Brown

USDA PLANTS (2018) cites Brown and Brown (1984), but there is no such attribution in Brown and Brown.

\section{Platanthera nivea (Nutt.) Luer}

Luer (1975) included Maryland in its range. This reported attribution to Maryland is erroneous and likely a misattribution based on its historical occurrence in Delaware.

Spiranthes laciniata (Small) Ames

Attributed to Maryland by Sheviak and Brown (2002), yet no specimen is known validating this attribution.

\section{OROBANCHACEAE}

(Broomrape Family)

Agalinis fasciculata (Elliott) Raf.

Attributed to the state by the Maryland Natural Heritage Program (2010) as historic, but all specimens believed to be this taxon are Agalinis purpurea.

\section{Agalinis linifolia (Nutt.) Britton}

[Gerardia linifolia Nutt.]

Attributed to Maryland by USDA PLANTS (2018), citing Brown and Brown (1984:871), who stated it is "rare; reaches northern limit in southern Delaware." Brown and Brown do not explicitly attribute this species to Maryland, and we have seen no vouchers from the state.

\section{PAPAVERACEAE}

(Poppy Family)

Corydalis aurea Willd.

Attributed to Maryland by Brown and Brown (1984), but no specimen can be found to validate this attribution.

\section{Papaver nudicaule L.}

Attributed by Brown and Brown (1984:488), stating “often occur as escapes," but no specimen is known to validate this claim.

\section{Papaver orientale L.}

Attributed by Brown and Brown (1984:488), stating “often occur as escapes," but no specimen is known to validate this claim.

\section{Papaver somniferum L.}

Attributed by Brown and Brown (1984:488), stating "often occur as escapes ... Harford County," but no specimen is known to validate this claim.

\section{PLANTAGINACEAE}

(Plantain Family)

Bacopa caroliniana (Walter) B. L. Rob.

[Hydrotrida caroliniana (Walter) Small]

Brown and Brown (1984:848) stated, "Rare; Coastal." No specimen can be found to support this report, and all other known reports for Maryland are based upon this vague attribution.
Digitalis lanata Ehrh.

Attributed by Brown and Brown (1984), but no specimen is known to validate this attribution.

\section{Digitalis lutea L.}

Attributed by Brown and Brown (1984), but no specimen is known to validate this attribution.

\section{Penstemon alluviorum Pennell}

Brown and Brown (1984:859) stated, "Reported for Baltimore County (Baltars); rare," but this species ranges only as far east as Alabama, Tennessee, and Kentucky.

\section{Penstemon calycosus Small}

Reported by Pennell (1935:215), stating "Maryland to Maine," and Brown and Brown (1984:859) state "Rare; in Baltimore County (Baltars)", but no specimen can be found validating these reports.

\section{Plantago beterophylla Nutt.}

Attributed by Morris (1909), but no specimen is cited to validate this attribution.

\section{Plantago maritima L.}

[Plantago maritima var. juncoides (Lam.) A. Gray]

A specimen at MARY, which is likely the source of attribution to Maryland, is misidentified.

\section{Plantago wrightiana Decne.}

Included by USDA PLANTS (2018), citing A. Radford et al., eds. Vascular flora of the southeastern United States (unpublished and undated). There is no date of publication and no treatment of Plantago was published in the Vascular flora of the southeastern United States. No specimen is known supporting this citation.

Veronica austriaca L. ssp. teucrium (L.) D. A. Webb

[Veronica latifolia L.]

Gleason (1952:236) stated, "w. to MD," but no specimen is known to validate this attribution. This is likely the source of all attribution to Maryland.

\section{Veronica beccabunga L.}

Attributed by numerous sources citing "1985 Rhodora." The paper being cited is by Les and Stuckey (1985), who cited a specimen from Harpers Ferry, West Virginia, but not Maryland.

\section{Veronica cymbalaria Bodard}

Attributed by Norton and Brown (1946), but searches have found no specimen validating this attribution. 


\section{POACEAE [Gramineae]}

(Grass Family)

Agrostis canina L.

Attribution stems from Brown and Brown (1984:137), stating "reported from Frederick Co., Md and Delaware." No specimen has been found validating this attribution.

\section{Agrostis mertensii Trin.}

[Agrostis borealis Hartm.]

Attributed by both Kartesz (2015a,b) and Terrell and Peterson (2009), citing Reed (1986), but the only citations for A. mertensii (as Agrostis borealis) are from Newfoundland and Quebec.

\section{Andropogon glaucopsis Elliott}

[Andropogon glomeratus (Walter) Britton, Sterns, \& Poggenb. var. glaucopis (Elliott) C. Mohr; A. virginicus L. var. dealbatus C. Mohr]

Attributed by Kartesz (2015a,b), citing a specimen at MARY. No such specimen can be found.

\section{Arundinaria gigantea (Walter) Muhl.}

Attribution is based upon confusion with Arundinaria tecta as modern treatments consider all Maryland material of the genus to be A. tecta.

\section{Bouteloua birsuta Lag.}

Gould (1979) mapped this species from either eastern Maryland or Delaware, and this map is the source of attribution in various databases. The location cannot be determined as no specimen is cited in the paper, and the report for either state cannot be validated without a specimen.

\section{Briza media L.}

Kartesz (2015a,b) and USDA PLANTS (2018) attributed this species to Maryland, citing Brown and Brown (1984). Brown and Brown (1984:168) stated, "Roadsides and swamps; rare; in Delaware." We suspect they were discussing the range and habitat in Delaware. There is no voucher specimen known to validate this species in Maryland.

\section{Bromus madritensis L.}

Attributed to Maryland by Kartesz (2015a,b), citing Brown and Brown (1984:147). Brown and Brown did not treat this species as a component of the flora and clearly stated that "Bromus madritensis has been cultivated as an ornamental."

\section{Bromus rubens $\mathrm{L}$.}

All attribution to this species in Maryland stems from Redmond (1932). No collection is known to exist supporting this report, and we expect it is erroneous.

\section{Cenchrus spinifex Cav.}

[Cenchrus incertus M. A. Curtis; C. pauciflorus Benth.]

Attribution stems from Norton and Brown (1946), as C. pauciflorus. No specimen is known to validate this report.
Coleataenia anceps (Michx.) Soreng ssp. rhizomata (Hitchc. \& Chase) Soreng

[Panicum anceps Michx. ssp. rhizomatum (Hitchc. \& Chase) Freckmann \& Lelong]

Attribution stems from Brown and Brown (1984:110), stating "occurs in Maryland," but no specimen can be found validating this report. Weakley (2015) reported this subspecies as far north as southeastern Virginia.

\section{Diarrhena americana P. Beauv.}

[Diarina festucoides Raf.]

Kartesz (2015a,b) cited the Maryland Natural Heritage Program for three counties in Maryland, but the Maryland Natural Heritage Program (C. Frye, Maryland Natural Heritage Program, personal communication) considers this plant to be "reported falsely." Attribution seems to stem from confusion with D. obovata.

Dichanthelium angustifolium (Elliott) Gould

[Panicum angustifolium Elliott]

Attributed by Weakley (2015), but no specimen has been seen validating this attribution.

\section{Dichanthelium arenicoloides (Ashe) LeBlond}

[Panicum arenicoloides Ashe]

Weakley (2015) attributed this species to western Maryland on the basis of Knapp 1374 (TAWES). We have annotated that specimen to D. linearifolium.

Dichanthelium boreale (Nash) Freckmann

[Panium boreale Nash]

Attributed by Hitchcock and Chase (1950), but specimens thought to be this taxon have been variously misidentified or are D. bicknellii.

Dichanthelium dichotomum (L.) Gould ssp. roanokense (Ashe) Freckmann \& Lelong

[Panicum dichotomum L. var. roanokense (Ashe) Lelong; P. roanokense Ashe]

Variously attributed to the flora by several authors, but no specimens have been found validating these attributions.

Dichanthelium ovale (Elliott) Gould \& C. A. Clark

[Panicum ovale Elliott]

Attributed based on confusion with D. commonsianum, but we consider the two distinct. Though $D$. commonsianum occurs in Maryland, D. ovale ranges farther south.

Elymus trachycaulus (Link) Gould ssp. glaucus (Pease \& A. H. Moore) Cody

[Agropyron trachycaulum (Link) Malte ex H. F. Lewis var. glaucum (Pease \& A. H. Moore) Malte]

Gleason (1952) included Maryland in the general range of this species, but no specimen is known to validate its presence in Maryland. 
Elymus trachycaulus (Link) Gould ssp. subsecundus (Link) Á. Löve \& D. Löve

[Agropyron subsecundum (Link) Hitchc.; A. trachycaulum (Link) Malte ex H. F. Lewis var. unilaterale (Cassidy) Malte] Attributed by Hitchoock and Chase (1950:238) (as A. subsecundum), citing "south to the mountains of Maryland." This reported attribution to Maryland is curiously identical to the report of Gleason (1952) of Agropyron trachycaulum var. glaucum. No voucher has been found validating this attribution.

\section{Glyceria fluitans (L.) R. Br.}

All attribution is based upon Shreve et al. (1910). No specimen is known to validate this report. Also reported from Baltimore County by Terrell and Peterson (2009).

\section{Glyceria grandis S. Watson, S1/G5}

Listed by the Maryland Natural Heritage Program (2018) as rare, but there is no specimen known to back this attribution. Though we consider this species as likely to be a component of the flora, this grass can be confused with other members of the genus and is excluded until sufficient evidence can be provided to validate its attribution.

\section{Hordeum brachyantherum Nevski ssp. brachyantherum}

[Hordeum nodosum L.]

Reported by Brown and Brown (1984) as Hordeum nodosum. We reiterate the conclusion of Shetler and Orli (2002) that this species is best excluded from the flora as its inclusion was based on confusion with $H$. nodosum or $H$. pratense Huds.

\section{Mublenbergia bushii Pohl}

[Mublenbergia brachyphylla Bush]

Hitchoock and Chase (1950:394) stated (as M. brachyphylla), "Low woods Maryland to NC." No specimen can be found to validate this report.

Mublenbergia racemosa (Michx.) Britton, Sterns, \& Poggenb.

This attribution is likely based upon taxonomic confusion with M. glomerata. Mublenbergia racemosa was reported by Gleason and Cronquist (1963) and repeated in the literature elsewhere. This reported attribution to Maryland is most likely based upon confusion with our single population of $M$. glomerata, which was considered a synonym of $M$. racemosa by Gleason and Cronquist (1963).

Paspalum setaceum Michx. var. villosissimum (Nash) Vasey

Brown and Brown (1984) attributed this species to Maryland, but most modern treatments (Allen and Hall 2003; Weakley 2015) consider this variety to be restricted to Florida and Cuba. This attribution is likely based upon taxonomic confusion.

\section{Phalaris caroliniana Walter}

Attributed by Brown and Brown (1984:117), stating "Coastal Plain; rare." No specimen is known to support this inclusion in the flora. This attribution seems to stem from Shreve et al. (1910), who cited a Robert K. Miller specimen from "Baltimore County, near Towson." The species is widely planted as a forage crop, but no specimen has been found to substantiate the record. Additionally, Maryland is not within the natural range of the species, which according to Weakley $(2015: 435)$ is "NC west to OR, south into Mexico, the original distribution now obscured." Any location in Maryland would be doubtlessly planted and is $\sim 500 \mathrm{~km}$ north of the northernmost station in North Carolina.

Phyllostachys flexuosa Rivière \& C. Rivière

USDA PLANTS (2018) and Kartesz (2015a,b) cited Brown and Brown (1984:196). Brown and Brown simply stated, "Has been reported for Frederick County." No specimen validating this attribution has been found.

Poa chapmaniana Scribn.

Reported by Stieber (1971:274) "at the border of a cornfield and in broken pavement at Java Farm." Stieber stated there is a specimen deposited at US, but searches for this specimen have revealed none. If this specimen was identified correctly, it was most likely a waif.

\section{Poa languida Hitchc.}

All citation is based on the literature (Fernald 1950), and the only specimen identified as P. languida (Monteferrante s.n. BALT) is $P$. saltuensis. Additionally, a specimen of $P$. languida at NY (Hill s.n.) with a handwritten label appearing to say "Whitting, Md" actually says "Whiting, Ind." This specimen may be the source of misattribution to the Maryland flora. See similar discussion under Linnaea borealis.

\section{Saccharum brevibarbe (Michx.) Pers. var. brevibarbe}

[Erianthus brevibarbis (Michx.) Pers. var. brevibarbis]

Attributed by Brown and Brown (1984:74), stating "Delaware and Somerset County (Broome); rare." This is likely the source of Terrell and Peterson's (2009) Somerset County attribution. No specimen can be found validating this report, and all specimens previously determined as such have been reidentified as Erianthus brevibarbis var. contortus.

\section{Zea mexicana (Schrad.) Kuntz}

Attribution stems from Stieber (1971:275), stating "escaped from cultivation, near fields, LCU." No specimen can be found validating this attribution. Perhaps best treated as a waif.

\section{Zizania palustris L. var. palustris}

[Zizania aquatica L. var. angustifolia Hitchc.]

Attributed by Redmond (1932) and Tucker (1988). No specimen is known supporting these attributions. 


\section{POLEMONIACEAE}

(Phlox Family)

Phlox carolina L.

[Phlox carolina ssp. angusta Wherry; P. carolina ssp. turritella Wherry]

Attribution of this species stems from Wherry (1931:33). Wherry stated, "In 1831 Aikin recorded from this state a Phlox revoluta, his description of it corresponding closely to the variety of $P$. carolina which ranges farthest northeastward. He apparently obtained it in Frederick County, although it has not subsequently been collected there." This species is here excluded because of the lack of a verifiable specimen.

\section{Phlox ovata L., SH/G4}

[Phlox latifolia Michx.]

Attributed by Brown and Brown (1984:767), stating "known Allegany County." Norton and Brown (1946) cited Wherry (1931:27); however, Wherry simply stated, "Reported by Shreve from Alleghany County. (1910)." Shreve et al. (1910:473) cited "Alleghany County, near Cumberland (George M. Perdew)" after his entry for P. ovata, which is likely a collection, but no specimen has been found validating this attribution.

\section{POLYGALACEAE}

(Milkwort Family)

\section{Polygala brevifolia Nutt.}

USDA PLANTS (2018) attributed this to Maryland, citing Brown and Brown (1984:615). Brown and Brown clearly stated, "No Maryland report" in this text for this species.

\section{Polygala cymosa Walters}

USDA PLANTS (2018) attributed this to Maryland, citing Brown and Brown (1984:615). Brown and Brown clearly stated, "No Maryland report."

\section{Polygala paniculata L.}

USDA PLANTS (2018) cited Brown and Brown (1984), but no record exists in Brown and Brown.

\section{Polygala ramosa Elliott}

Brown and Brown (1984:615) stated "Maryland, Delaware and New Jersey; locally abundant." This reported attribution to Maryland is erroneous. There is no previous attribution of this plant to Maryland and no known specimen from the state. The source for this error is likely that the plant is historically known from nearby Delaware.

\section{POLYGONACEAE}

(Buckwheat Family)

\section{Fallopia baldschuanica (Regel) Holub}

[Polygonum baldschuanicum Regel]

Attributed to Maryland by Hinds and Freeman (2005), but Craig C. Freeman (University of Kansas, personal communication) knows of no specimen validating this attribution. Hinds passed away before the Fallopia treatment of FNA was completed, and it is possible he saw specimens supporting his report.
USDA PLANTS (2018) cited a specimen at NCU, but searches at NCU have found no specimens (C. A. McCormick, University of North Carolina-Chapel Hill, personal communication).

Fallopia dumetorum (L.) Holub

[Polygonum scandens L. var. dumetorum (L.) Gleason]

Attributed by Kartesz (2015a,b) and Hinds and Freeman (2005). Kartesz cited a specimen at MARY, but this specimen has been annotated to F. cristata. No specimen can be found validating the Hinds and Freeman (2005) attribution, and Freeman (pers. comm.) knows of no specimen validating this attribution. Hinds passed away before the Fallopia treatment of FNA was completed, and it is possible he saw specimens validating his report.

Persicaria careyi (Olney) Greene

[Polygonum careyi Olney]

Attributed by Anderson et al. (1965) and repeated by Brown and Brown (1984:408), stating "Upper Patuxent estuary, Anne Arundel County; also Delaware. Rare.” No specimen is known to support this report.

Persicaria chinensis (L.) H. Gross

Attributed to Maryland by Hinds and Freeman (2005), but Freeman (pers. comm.) knowns of no specimen validating this attribution. Hinds passed away before the Persicaria treatment of FNA was completed, and it is possible he saw specimens validating his report.

Rumex fueginus Phil.

[Rumex maritimus L.]

Attributed by Brown and Brown (1984:399), stating "infrequent," but no specimen has been found validating this attribution.

\section{Rumex sanguineus L.}

Reported from Maryland by Kartesz (1999), but no evidence is known validating this attribution.

\section{POTAMOGETONACEAE}

(Pondweed Family)

Potamogeton obtusifolius Mert. \& W. J. D. Koch

Attributed to Maryland by USDA PLANTS (2018), citing "Rhodora 1974." This citation is in reference to Haynes (1974:605), yet in that paper there is no attribution of this species to Maryland, and the range cited says "south ... [ [to] New Jersey." Furthermore, Haynes and Hellquist (2002) did not attribute this species to Maryland.

\section{Potamogeton praelongus Wulfen}

First attributed by Haynes (1985) but later not included from Maryland in his Flora of North America treatment of the genus (Haynes and Hellquist 2002). No specimen has been seen validating the 1985 attribution. 
Potamogeton richardsonii (A. Benn.) Rydb.

Attributed by numerous sources, but all specimens identified as such have been annotated as $P$. crispus or P. perfoliatus.

Potamogeton spirillus Tuck.

Listed as a rare species by the Maryland Natural Heritage Program (2010), but all specimens found initially identified as $P$. spirillus have been other Potamogeton species, usually P. diversifolius. USDA PLANTS (2018) and Kartesz (2015a,b) attributed the species to the state, citing Brown and Brown (1984:48). Brown and Brown stated, "Shallow smooth waters. In Delaware; rare." We think Brown and Brown were not stating this species was in Maryland but instead saying it is known from Delaware.

\section{RANUNCULACEAE (Buttercup/Crowfoot Family)}

Anemone minima DC.

Attributed by Brown and Brown (1984:474), stating "mountain zone." No specimen is known supporting this attribution.

Anemone virginiana L. var. alba (Oakes) Alph. Wood

[Anemone riparia Fernald]

Attributed by Brown and Brown (1984), but no specimen is known to validate this attribution.

\section{Consolida regalis Gray}

[Delphinium consolida L.]

Attributed by Brown and Brown (1984:479), stating "mostly in cultivated soils. Widely common (PLM) but not abundant." Kartesz $(2015 \mathrm{a}, \mathrm{b})$ cited a specimen at MARY. Searches at MARY have found no specimens validating these attributions.

\section{Ranunculus arvensis $\mathrm{L}$.}

Attributed by Hultén and Fries (1986) with a mapped dot in either southern Maryland or Virginia, but no specimen is known validating this attribution.

\section{Ranunculus trichophyllus Chaix var. trichophyllus, S1/G5}

[Ranunculus aquatilis L.]

Attributed by the Maryland Natural Heritage Program (2018), but no specimen is known to validate this attribution. This report is likely based upon a misidentified Cabomba carolinensis.

\section{Thalictrum dasycarpum Fischer \& Avé-Lall.}

Attributed to Maryland by Brown and Brown (1984:470), stating "in the W-B [Washington-Baltimore] area; infrequent." No specimen is known to support this claim, and Brown and Brown is likely the source of all other known attributions.

Thalictrum macrostylum Small \& A. Heller

[Thalictrum subrotundum B. Boivin]

Attributed to Maryland by Brown and Brown (1984), where they atypically gave no habitat or region within Maryland for this species. No specimen is known to support this claim, and Brown and Brown is likely the source of all other known attributions.

\section{RHAMNACEAE}

(Buckthorn Family)

Berchemia scandens (Hill) K. Koch

Attributed by various sources, but all known specimens document the northern limit of this species as Accomack County, Virginia. This species very likely could occur in Maryland as it is in close proximity on Chincoteague Island, Virginia.

\section{Rhamnus cathartica L.}

Attributed by Brown and Brown (1972). All specimens found identified as this species have been misidentifications.

\section{Rhamnus lanceolata Pursh ssp. lanceolata}

Initially attributed by Johnston (1975) and the likely source for all future attribution. Johnston cited a specimen, Lyon s.n. (PH), but searches at $\mathrm{PH}$ have found no such specimen. It is either lost or has been reidentified.

ROSACEAE

(Rose Family)

Aphanes microcarpa (Boiss. \& Reut.) Rothm

[Alchemilla microcarpa Boiss. \& Reut.]

Variously attributed to the flora. All our plants are A. australis, as A. microcarpa is a Mediterranean species.

Argentina anserina (L.) Rydb.

[Potentilla anserina L.]

Attributed by Hitchcock and Standley (1919), but no specimen is known to validate this attribution.

\section{Crataegus macracantha Lodd. ex Loudon}

Attributed by Lance (2014) and Phipps (2014) and likely to be found in Maryland. No specimen could be found validating this attribution.

\section{Crataegus margarettae Ashe}

Attributed by Brown and Brown (1972:152), stating "Dan's Mountain, Allegany County." No specimen can be found validating this claim. Recent treatments are split on its presence in Maryland. Lance (2014) attributed it, but Phipps (2014) did not. It has potential to be found in Maryland given its distribution in adjacent states.

\section{Crataegus populnea Ashe}

[Crataegus iracunda Beadle var. populnea (Ashe) Kruschke] Reported by Brown and Brown (1972) and Lance (2014), but no voucher is known.

Crataegus schuettei Ashe var. schuettei

[Crataegus alnorum Sarg.; C. basilica Beadle; C. ferrissii Ashe;

C. silvicola Beadle; C. stolonifera Sarg.; C. tortilis Ashe] 
Attributed by Phipps (2014) and is potential in western

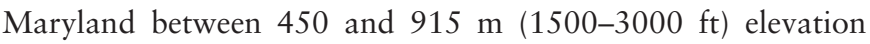
(J. B. Phipps, University of Western Ontario, personal communication). Though a likely component of the flora, no specimen could be found validating this attribution.

Gillenia stipulata (Muhl. ex Willd.) Nutt.

[Porteranthus stipulatus (Muhl. ex Willd.) Britton]

Attributed by various sources, but the only specimen previously identified as such, Reynard s.n. MARY, is G. trifoliata.

Malus baccata (L.) Borkh.

Variously attributed by different sources and a likely component of our flora given its distribution in Delaware and Virginia. No specimen is known to validate attribution in Maryland.

Malus ioensis (Alph. Wood) Britton var. ioensis

Attributed by Kartesz (2015a,b), citing a specimen at MARY, but searches at MARY have found no such specimen.

Rosa luciae Franch. \& Rochebr.

Attributed by Kartesz (2015a,b) and Lewis et al. (2014). No specimen is known validating this attribution. If one is found, this species might be best treated as a waif.

\section{Rubus trivialis Michx.}

[Rubus continentalis (Focke) L. H. Bailey; R. rubrisetus Rydb.]

Brown and Brown (1972:164) stated the species "is reported from the Coastal Plain of Maryland" but no specimen is known to validate this attribution. The citation by the Browns could be a broad one and not an attribution to the Maryland flora, as this was reported from the Coastal Plain of Virginia by Tatnall (1946).

\section{RUBIACEAE}

(Madder/Coffee Family)

Galium odoratum (L.) Scop.

[Asperula odorata L.]

Attributed by USDA PLANTS (2018), citing Brown and Brown (1984:892). Brown and Brown stated, "No Maryland record but reported from Delaware."

\section{Galium palustre L.}

Attributed by Kartesz (2015a,b) and USDA PLANTS (2018), citing the "Maryland Heritage and Biodiversity Conservation Program." The initial source of this attribution is unknown, and no specimen is known confirming this species for the state.

\section{Galium trifidum L. var. trifidum}

All specimens seen previously identified as Galium trifidum are misidentifications of various other Galium species.

\section{Galium uniflorum Michx.}

Attributed by Kartesz (2015a,b), citing a specimen at MARY. The single sheet upon which the attribution is based is not correctly identified and is instead in the G. trifidum complex.

\section{Houstonia lanceolata (Poir.) Britton}

[Houstonia purpurea L. var. calycosa A. Gray]

Tatnall (1946:246) stated, "One collection: woods 2 mi. s. of Easton," and cited a specimen, J. A. Jump, 3 June 1937, housed at PH. Searches at PH have found no specimen. It is possible the specimen's identification has been changed.

\section{Houstonia longifolia Gaertn. var. longifolia}

Attributed by Brown and Brown (1984), but this species is known to range only as far south as southern New Jersey.

\section{RUTACEAE}

(Citrus/Rue Family)

Ruta graveolens $\mathrm{L}$.

All specimens seen were from cultivated plants.

\section{SALICACEAE}

(Willow Family)

Salix amygdaloides Andersson

Attributed by Witman (1954), but no specimen is known validating this attribution.

\section{Salix caprea L.}

Attributed by Brown and Brown (1984:34), stating "the species is propagated by cuttings rarely escapes," but no specimen has been found outside cultivation. Kartesz (2015a,b) attributed this species to numerous counties, but no specimens can be found validating this attribution.

\section{Salix elaeagnos Scop.}

[Salix incana Schrank]

Attributed by USDA PLANTS (2018), citing Brown and Brown (1984). This is a curious citation since the woody plants are in Brown and Brown (1972). Neither the 1984 nor 1972 volume contains a mention of Salix elaeagnos or its synonyms.

\section{Salix triandra L.}

Attributed by Kartesz (2015a,b), citing "Harvard Papers in Botany 2007." Argus (2007) mapped the distribution of the genus at such a scale that it is difficult to determine if the location being mapping is Washington, D.C., or Maryland. We think this mapped location is Washington, D.C. No voucher has been found to validate the attribution of Washington, D.C., or Maryland.

\section{SAPINDACEAE}

(Maple Family)

\section{Acer campestre L.}

Attributed by Witman (1954), yet no specimen exists validating this treatment. If found in Maryland, it is likely a waif. 


\section{SAPOTACEAE}

(Sapodilla Family)

\section{Sideroxylon lycioides $\mathrm{L}$.}

[Bumelia lycioides (L.) Pers.]

Attributed to Maryland by USDA PLANTS (2018), citing "Radford et al. Flora of the Southeastern United States." No specimen is known supporting this attribution.

\section{SAURURACEAE}

(Lizard's-tail Family)

Houttuynia cordata Thunb.

Attributed by Kartesz (2015b) but not currently known to be established. This is an invasive species in adjacent Pennsylvania and Virginia. All Maryland specimens seen have originated from cultivation.

\section{SAXIFRAGACEAE}

(Saxifrage Family)

Astilbe biternata (Vent.) Britton

Attributed to Maryland by Brown and Brown (1984:534), stating "Moist woods; rare." No specimen is known to support this attribution.

\section{Heuchera villosa Michx. var. villosa}

[Heuchera macrorhiza Small]

All attribution stems from Robinson and Fernald (1908). Searches at herbaria, including GH, have yielded no specimen validating this report.

\section{Micranthes petiolaris (Raf.) Brouillet \& Gornall}

[Hydatica petiolaris (Raf.) Small; Saxifraga michauxii Britton]

Reported by Kartesz (2015a,b) and USDA PLANTS (2018), citing "Journal of the Arnold Arboretum (1972)." In this paper Spongberg (1972:445) stated, "In the Appalachians from Maryland to northwest Georgia." No specimen is known validating its presence in Maryland.

\section{SOLANACEAE}

(Nightshade/Potato Family)

\section{Nicotiana longiflora Cav.}

Attributed by Brown and Brown (1984), but all specimens seen have come directly from cultivation.

\section{Physalis grisea (Waterf.) M. Martínez}

Attributed by Brown and Brown (1984) as P. pruinosa L., but that is not the correct name for North American plants (Weakley 2015). This is the only source of attribution from the state, and no specimen is known validating this attribution.

\section{Solanum americanum Mill.}

[Solanum nodiflorum Jacq.; S. ptychanthum Dunal]

Attributed by Brown and Brown (1984:834), stating "Reported from Baltimore County (Baltars)." This species does not occur north of northern Georgia. Reed (1964) reported
S. nodiflorum Jacq. (=S. americanum?), but because we have not seen his voucher, the application of this name is not clear.

Solanum sarrachoides Sendtn.

The first apparent attribution is Stieber (1971:302), stating "Weed of gardens and waste places, US." There is no specimen at US supporting this attribution, and it is likely the specimen he cited has been annotated to another species.

TOFIELDIACEAE

(False Asphodel Family)

Triantha glutinosa (Michx.) Baker

[Tofieldia glutinosa (Michx.) Pers.]

Attributed to Maryland by Brown and Brown (1984), but no specimen is known to verify this attribution. No source, other than those citing Brown and Brown, attributes this species to Maryland.

ULMACEAE

(Elm Family)

Ulmus glabra Huds.

Variously attributed, but there is no specimen establishing its presence beyond cultivation.

Ulmus minor Mill.

[Ulmus procera Salisb.]

Attributed by Brown and Brown (1972), but there is no evidence this is establishing beyond cultivation.

Ulmus thomasii Sarg.

A widely planted ornamental. There is no evidence that this species is escaping or has established itself outside of cultivation.

\section{URTICACEAE}

(Nettle Family)

Boehmeria nivea (L.) Gaudichaud-Beaupré

Brown and Brown (1984:392) said that it is "reported for Maryland" but gave no further attribution. Kartesz (2015a,b) attributed a specimen at MARY, but the specimen is clearly of cultivated origin (Hill 12484 MARY).

\section{Parietaria floridana Nutt.}

Kartesz (2015a,b) attributed the species to Prince George's County and cited David Boufford as the best source. Boufford (Harvard University Herbaria, personal communication) stated he saw a specimen from the "vicinity of USDA greenhouses." If a specimen is found, it is likely to be considered a waif in Maryland.

\section{Parietaria judaica L.}

Kartesz (2015a,b) attributed David Boufford, personal communication. Boufford did not have a record of the herbarium of deposit but stated he saw a specimen from Govans, Maryland. There is likely a specimen at a herbarium not surveyed for this project. If a specimen is found, it is likely to be considered a waif in Maryland. 


\section{Urtica urens $\mathrm{L}$.}

Attributed by Brown and Brown (1984:392), stating "Naturalized; still uncommon.” There is no specimen known validating this attribution. Perhaps this species is best treated as a waif.

\section{VERBENACEAE}

(Verbena Family)

Verbena neomexicana (A. Gray) Small

Reported by Gleason and Cronquist (1963), but this attribution cannot be substantiated with a specimen. If this species was collected in Maryland, it most likely was a waif and not established.

\section{VIOLACEAE}

(Violet Family)

Viola patrinii DC.

Attributed to Maryland by Kartesz (2015a,b) and USDA PLANTS (2018), citing “1946 Castanea.” This citation is likely Norton and Brown (1946), but there is no citation of this species or its synonyms contained here.

\section{Viola septemloba Leconte}

Attribution is based upon confusion of the name in the Northeast. This species is not known to range farther north than southeastern Virginia and is in pyrophytic wiregrass-pine savannas (H. Ballard, Ohio University, personal communication).

\section{Viola septentrionalis Greene}

Brown and Brown (1984:657) stated, "Cranesville Swamp, Garrett County." Though this location would make biogeographic sense, there is no specimen validating this attribution.

\section{Viola tripartita Elliott}

[Viola tripartita var. glaberrima (Ging.) R. M. Harper]

USDA PLANTS (2018) cited "Radford, et al. (eds.) Vascular flora of the southeastern United States. Unpublished and undated." This attribution is backed by no specimen, and the citation itself is problematic as it is unpublished.
VITACEAE

(Grape Family)

Ampelopsis cordata Michx.

No known specimen exists to validate the attribution of this species to Maryland outside of cultivation. This attribution may be based upon early collections and misidentifications of Ampelopsis glandulosa.

Nekemias arborea (L.) J. Wen \& Boggan

[Ampelopsis arborea (L.) Koehne]

Attributed to Maryland by Brown and Brown (1972:225), stating "Swampy woods, southeastern part of state," but no known specimen exists to validate this attribution.

Parthenocissus inserta (A. Kern) Fritsch

[Parthenocissus quinquefolia (L.) Planch var. vitacea Knerr; P. vitacea (Knerr) Hitchc.]

Attribution stems from Moore and Wen (2016), but no specimen is known to validate this attribution. Its presence is expected in Maryland given this species is known and has been attributed from adjacent Delaware, Pennsylvania, and Virginia.

Parthenocissus tricuspidata (Siebold \& Zucc.) Planch.

Attributed by Kartesz (2015a,b), citing a specimen at GA, and attributed by USDA PLANTS (2018), stating "Michael O. Moore pers. comm." No specimen was found at GA, where Michael O. Moore worked before his death. This attribution based upon a personal communication cannot be validated further and is excluded.

XYRIDACEAE

(Yellow-eyed Grass Family)

\section{Xyris caroliniana Walter}

Attribution is based upon confusion with other Xyris species. In the region, Xyris caroliniana is known from a single location in New Jersey and southeastern Virginia. 


\section{Acknowledgments}

$\mathrm{T}$

This project has been a labor of love and has required many years of dedicated effort. Much of this project was completed on the personal time of the first author, who expresses the deepest gratitude to his wife, Heather, and daughters, Sidney and Bella, for their patience and assistance in family-oriented outings to document the flora of Maryland.

The knowledge represented in this checklist would not be present without all those who worked to study and document the flora before this point. Though the number of experts who have worked to understand the flora is far too great to list in entirety, we would like to thank the following experts for their input, efforts, and/or contributions to the knowledge of the Maryland flora: Lance Biechele, Daniel Boone, Jim Brighton, Allison Cusick, Charles Davis, Cris Fleming, Gary Fleming, Christopher Frye, Steve Grund, John Hall, Bill Harms, Jason Harrison, Frank Hirst, Bill Hubick, Kerrie Kyde, Roger Latham, Christopher Lea, Wayne Longbottom, Christopher Ludwig, William McAvoy, Kathy McCarthy, Joe Metzger, John Parrish, Jim Reveal, Richard Riefner, Rod Simmons, Peter Stango III, Jim Stasz, Brent Steury, Mark Strong, Jil Swearingen, Ed Thompson, Johnny Townsend, Amanda Treher, Arthur Tucker, Wayne Tyndall, Alan Whittemore, Richard Wiegand, Ron Wilson, and Kerry Wixted.

We deeply thank experts in various taxonomic groups for their input, evaluation, and comments: Harvey Ballard (Viola), Paul Martin Brown (various Orchidaceae), David Boufford (Parietaria), Judith Canne-Hilliker (Agalinis), Patricia Cox (Rudbeckia), Aaron Floden (Helianthus and Trillium), Craig Freeman (Persicaria), Doug Goldman (Rubus), Robert Haynes (Potamogeton), John Hall (Lemna and numerous other aquatic genera), Steven Hill (Malvaceae), Charles Horn (Heteranthera), Ron Lance (Crataegus), Richard LeBlond (Dichanthelium), John Nelson (Stachys), James Phipps (Crataegus), Paul Peterson (Poaceae), Derick Poindexter (Scutellaria), Hamid Razifard (Elatine), Anton Reznicek (Carex polymorpha), Edward Schilling (Helianthus), Ernie Schuyler (Scirpus and Schoenoplectus), Robin Scribailo (Myriophyllum), John Semple (Doellingeria and Solidago), Charles Sheviak (various Orchidaceae), Galen Smith (Schoenoplectus), Robert Soreng (Poaceae), Bruce Sorrie (Doellingeria, Lechea, and Linum), Carl Taylor (Gymnocarpium), Gordon Tucker (Zizania), Alan Whittemore (Ranunculus), and John Wiersema (Nymphoides).

We also greatly appreciate the assistance of numerous herbarium staff and curators for searching their holdings for Maryland specimens of various taxa: Elana Benamy $(\mathrm{PH})$, Anthony Brach (GH), Sunshine Brosi (Frostburg State University), Johnathan 
Chase (Assateague Island National Seashore Herbarium), Tim Dickenson (TRT), Donna Ford-Werntz (WVA), Brian Franzone (GH), Christopher Frye (TAWES), John Hall (MARY), David Heard (BALT), Cynthia Hong-Wa (DOV), James Hull (BALT), Bonnie Isaac (CM), Tatyana Livshultz (PH), Carol Anne McCormick (NCU), Roland Roberts (BALT), Rusty Russell (US), Mark Strong (US), Alan Weakley (NCU), Robert Webster (NA), Brenda Wichmann (GA), Alan Whittemore (NA), George Yatskievych (MO), and Susan Yost (DOV).

We thank Jim Brighton and Bill Hubick of the Maryland Biodiversity Project/Maryland Plant Atlas, who were instrumental in calculating the statistics about the flora and housing photo vouchers for numerous records.

We thank Doug Goldman and Gerry Moore of the USDA PLANTS Database and John Kartesz and Misako Nishino of the Biota of North America Project for their data request assistance.

The manuscript was greatly improved by the reviews of Bill Buck, Chris Frye, Paul Peterson, Robert Soreng, and Alan Weakley.

The first author is also grateful to the Carnegie Museum of Natural History, which generously funded travel for reference specimen examination through the W. E. Buker Travel Award. 


\section{References}

Allen, C. M., and D. W. Hall. 2003. Paspalum. Fl. N. Amer. 25: 566-599.

Anderson, R. R., C. C. Phillip, R. D. Rappleye, and R. B. Brown. 1965. Checklist of Vascular Aquatic Plants on the Upper Patuxent Estuary. Contribution 3707, Miscellaneous Publication 556. Maryland Agricultural Experiment Station, Department of Botany, College Park.

Angiosperm Phylogeny Group. 2016. An Update of the Angiosperm Phylogeny Group Classification for the Orders and Families of Flowering Plants: APG IV. Bot. J. Linn. Soc. 181: 1-20. https://doi.org/10.1111/boj.12385

Argus, G. W. 2007. Salix (Salicaceae) distribution maps and a synopsis of their classification in North America, North of Mexico. Harvard Pap. Bot. 12(2): 335-368. https://doi.org/10.3100/1043-4534(2007)12[335:SSD MAA]2.0.CO;2

Benedict, J. E., Jr. 1924. An Occurrence of Southern Maiden-Hair in Maryland. Amer. Fern J. 14: 21-24. https:// doi.org/10.2307/1544376

Brooks, R. E., and S. E. Clemants. 2000. Juncaceae. Fl. N. Amer. 22: 225-255.

Broome, C. R., A. O. Tucker, N. H. Dill, and J. L. Reveal. 1979. Rare and Endangered Vascular Plant Species in Maryland. U.S. Fish and Wildlife Service, Newton Corner, Mass.

Brouillet, L. 2006. Tripleurospermum. Fl. N. Amer. 19: 548-551.

Brown, M. L., and R. G. Brown. 1984. Herbaceous Plants of Maryland. Book Center, University of Maryland, College Park.

Brown, M. L., J. L. Reveal, C. R. Broome, and G. F. Frick. 1987. Comments on the Vegetation of Colonial Maryland. Huntia 7: 247-283.

Brown, R. G., and M. L. Brown. 1972. Woody Plants of Maryland. Student Supply Store, University of Maryland, College Park.

Brummitt, R. K., and C. E. Powell, eds. 1992. Authors of Plant Names. Royal Botanic Gardens, Kew, Richmond, Surrey, UK

Cusick, A. 1986. Significant Additions to the Vascular Flora of Western Maryland. Castanea 5: 129-136.

Fernald, M. L. 1950. Gray's Manual of Botany. 8th ed. American Book Co., New York.

Flora of North America Editorial Committee, eds. 1993-. Flora of North America North of Mexico. Oxford University Press, New York.

Frye, C. T., and C. Lea. 2001. Atlas and Annotated List of Carex (Cyperaceae) of Maryland and the District of Columbia. Maryland Naturalist 44: 41-108.

Gleason, H. A. 1952. The New Britton and Brown Illustrated Flora of the Northeastern United States and Adjacent Canada. 3 vols. New York Botanical Garden, New York.

Gleason, H. A., and A. Cronquist. 1963. Manual of Vascular Plants of Northeastern United States and Adjacent Canada. Van Nostrand, Princeton, N.J.

Gould, F. W. 1979. The Genus Bouteloua (Poaceae). Ann. Missouri Bot. Gard. 66: 348-416. https://doi .org/10.2307/2398834

Harned, J. E. 1936. Wild Flowers of the Alleghanies. 2nd ed. Published by the author, Oakland, Md. https://doi .org/10.2307/4031027

Harrison, J. W. 2016. The Natural Communities of Maryland: 2016 Natural Community Classification Framework. Natural Heritage Program, Wildlife and Heritage Service, Maryland Department of Natural Resources, Annapolis.

Haynes, R. R. 1974. A Revision of North American Potamogeton Subsection Pusilli (Potamogetonaceae). Rhodora 76: 564-649.

Haynes, R. R. 1985. A Revision of the Clasping-Leaved Potamogeton (Potamogetonaceae). Sida 11: 173-188.

Haynes, R. R., and C. B. Hellquist. 2002. Potamogetaceae. Fl. N. Amer. 22: 48-70. 
Hermann, F. J. 1941. A Checklist of Plants in the Washington-Baltimore Area. Conference on District Flora, Smithsonian Institution, Washington, D.C.

Hermann, F. J. 1946. A Checklist of Plants in the Washington-Baltimore Area. 2nd ed. Conference on District Flora, Smithsonian Institution, Washington, D.C.

Hickey, C. J. 1975. The Vascular Flora of Catoctin Mountain Park, Frederick County, MD. M.S. thesis, Towson State College, Towson, Md.

Hill, S. R. 2016. Althaea. Fl. N. Amer. 6: 231-233.

Hinds, H. R., and C. C. Freeman. 2005. Persicaria. Fl. N. Amer. 5: 574-594.

Hitchcock, A. S., and A. Chase. 1950. Manual of the grasses of the United States, second edition. U.S. Dept. of Agriculture Miscellaneous Publication 200: 1-1051. https://doi.org/10.5962/bhl.title.65332

Hitchcock, A. S., and P. C. Standley. 1919. Flora of the District of Columbia and vicinity. Contr. U. S. Natl. Herb. 21: 1-329. https://doi.org/10.5479/10088/27003

Hultén, E. 1958. The amphi-Atlantic plants and their phytogeographic connections. Svenska Vetenskapsakademies Handl., Stockholm 7: 1-340.

Hultén, E., and M. Fries. 1986. Atlas of North European Vascular Plants North of the Tropic of Cancer. 3 vols. Koeltz Scientific Books, Konigstein, Germany.

Isely, D. 1990. Vascular Flora of the Southeastern United States. Volume 3, Part 2: Leguminosae (Fabaceae). University of North Carolina Press, Chapel Hill.

Johnston, L. A. 1975. Revision of the Rhamnus serrata complex. Sida 6: 67-97.

Judd, W. S., and K. A. Kron 2009. Rhododendron. Fl. N. Amer. 8: 455-473.

Kalm, P. 1772. Travels into North America. 2 vols. Translated by J. R. Foster. W. Eyres, Warrington Publishing. London, England.

Kartesz, J. T. 1999. A Synonymized Checklist and Atlas with Biological Attributes for the Vascular Flora of the United States, Canada, and Greenland. In Synthesis of the North American Flora, Version 1.0, ed. J. T. Kartesz and C. A. Meacham. North Carolina Botanical Garden, Chapel Hill. CD-ROM.

Kartesz, J. T. 2015a. Floristic Synthesis of North America, Version 1.0. Biota of North America Program (BONAP). Chapel Hill, N.C.

Kartesz, J. T. 2015b. North American Plant Atlas. The Biota of North America Program (BONAP). Chapel Hill, N.C. [Maps generated from Kartesz, 2015a.] (http://bonap.net/napa; accessed 19 February 2020).

Knapp, W. M. 2014. Juncus fascinatus (Juncaceae), a New Combination in Juncus Sect. Ozophyllum and Notes on Morphologically Similar Species. Phytotaxa 174: 243-260. https://doi.org/10.11646/phytotaxa.174.5.1

Knapp, W. M., R. F. C. Naczi, W. D. Longbottom, C. A. Davis, W. A. McAvoy, C. T. Frye, J. W. Harrison, and P. Stango III. 2011. Floristic Discoveries in Delaware, Maryland, and Virginia. Phytoneuron 64: 1-26.

Knapp, W. M., D. B. Poindexter, and A. S. Weakley. 2020. The True Identity of Marshallia grandiflora, an Extinct Species, and The description of Marshallia pulchra (Asteraceae, Helenieae, Marshalliinae). Phytotaxa 447: 001-015. https://doi.org/10.11646/phytotaxa.447.1.1

Koerber, W. A. 1962. Pteris cretica in Maryland. Castanea 27: 181.

Kott, L. S., and D. M. Britton. 1982. A Comparative Study of Sporophyte Germination of Some Isoetes Species of Northeastern North America. Canad. J. Bot. 60: 1679-1687. https://doi.org/10.1139/b82-218

Kral, R., and P. E. Bostick. 1969. The Genus Rhexia (Melastomataceae). Sida 3: $387-440$.

Lance, R. 2014. A Guide to the Hawthorns of the Southeastern United States. Published by the author, Mills River, N.C.

Les, D. H., and R. L. Stuckey. 1985. The Introduction and Spread of Veronica beccabunga (Scrophulariaceae) in Eastern North America. Rhodora 87: 503-515.

Lewis, W. H., B. Ertter, and A. Bruneau. 2014. Rosa. Fl. N. Amer. 9: 75-119.

Longbottom, W., R. F. C. Naczi, and W. M. Knapp. 2016. Flowering Plant Species New to Delaware and Maryland. Bartonia 69: 5-19.

Luer, C. A. 1975. The Native Orchids of the United States and Canada Excluding Florida. New York Botanical Garden, New York.

Luttman, J. F. 1935. Contribution to the Flora of Garrett County, MD.: A Physioecological Study of the Flora of Garrett County. Master's thesis, Catholic University of America, Washington, D.C.

Magrath, L. K., and J. V. Freudenstein. 2002. Corallorhiza. Fl. N. Amer. 26: 633-638.

Maryland Natural Heritage Program. 2010. Rare, Threatened, and Endangered Plants of Maryland. Natural Heritage Program, Wildlife and Heritage Service, Maryland Department of Natural Resources, Annapolis.

Maryland Natural Heritage Program. 2018. Rare, Threatened, and Endangered Plants of Maryland. Natural Heritage Program, Wildlife and Heritage Service, Maryland Department of Natural Resources, Annapolis. http://dnr.maryland. gov/wildlife/Documents/rte_Plant_List.pdf (accessed 11 December 2018).

Maryland Plant Atlas Work Group. 2016. Digital Atlas of the Maryland Flora. https://MarylandPlantAtlas.org (accessed 15 May 2020).
McAtee, W. L. 1930. Seventh Supplement to the Flora of the District of Columbia and Vicinity. Proc. Biol. Soc. Wash. 43: 21-54.

Moldenke, H. N. 1942. Noteworthy Plant Records and Nomenclatural Notes. Castanea 7: 123-125.

Moore, M. O., and J. Wen. 2016. Parthenocissus. Fl. N. Amer. 12: 16-17.

Morris, E. L. 1909. North American Plantaginaceae-III. Bull. Torrey Bot. Club. 36: 515-530. https://doi.org/10.2307/2479291

Morton, J. K. 2005. Stellaria. Fl. N. Amer. 2: 96-114.

Morton, J. K., and R. K. Rabeler. 1989. Biosystematic Study of the Stellaria calycantha (Carophyllaceae) Complex. I. Cytology and Cytogeography. Canad. J. Bot. 67: 121-127. https://doi.org/10.1139/b89-018

Naczi, R. F. C. 2016. New Manual of Vascular Plants of Northeastern United States and Adjacent Canada. Brittonia 68: 238-244. https://doi.org/10.1007/ s12228-016-9416-6

Naczi, R. F. C., and Collaborators. 2016 [and onward]. New Manual of Vascular Plants of Northeastern United States and Adjacent Canada. Online edition. NYBG Press, New York. http://sweetgum.nybg.org/science/projects/northeastflora/ (accessed 1 May 2020).

Norton, J. B. S. 1930. Maryland Grasses. Maryland Agric. Exp. Sta. Bull. 323: 215-326.

Norton, J. B. S., and R. G. Brown. 1946. A Catalog of the Vascular Plants of Maryland. University of Maryland Agricultural Experimental Station, College Park.

Olsen, R. T., and J. H. Kirkbride Jr. 2017. Taxonomic Revision of the Genus Catalpa (Bignoniaceae). Brittonia 69: 387-421. https://doi.org/10.1007/ s12228-017-9471-7

Park, K. 1998. Monograph of Euphorbia Sect. Tithymalopsis (Euphorbiaceae). Edinburgh J. Bot. 55: 161-208. https://doi.org/10.1017/S0960428600002122

Pennell, F. W. 1935. The Scrophulariaceae of Eastern Temperate North America. Acad. Nat. Sci. Philadelphia Monogr. 1: 1-650.

Phipps, J. B. 2014. Crataegus. Fl. N. Amer. 9: 491-643.

Pryer, K. M. 1993. Gymnocarpium. Fl. N. Amer. 2: 258-262.

Pryer, K. M., and C. H. Haufler. 1993. Isozymic and Chromosomal Evidence for the Allotetraploid Origin of Gymnocarpium dryopteris (Dryopteridaceae). Syst. Bot. 18: 150-172. https://doi.org/10.2307/2419795

Redman, D. E. 1991. An Annotated List of the Ferns and Fern Allies of Maryland and the District of Columbia. Maryland Naturalist 35: 15-24.

Redmond, P. J. 1932. A Flora of Worcester County, Maryland. Ph.D. diss., Catholic University, Washington, D.C.

Reed, C. C. 1943. County Distribution of the Ferns and Fern-Allies in Maryland, Delaware and the District of Columbia. Bull. Nat. Hist. Soc. Maryland 13:47

Reed, C. F. 1964. A Flora of the Chrome and Manganese Ore Piles at Canton, in the Port of Baltimore, Maryland and at Newport News, Virginia, with Descriptions of Genera and Species New to the Flora of Eastern United States. Phytologia 10: 324-406. https://doi.org/10.5962/bhl.part.8346

Reed, C. F. 1986. Floras of the Serpentine Formations in Eastern North America, with Descriptions of Geomorphology and Mineralogy of the Formations. Reed Herbarium, Baltimore.

Reveal, J. L., C. R. Broome, M. L. Brown, and G. F. Fink. 1987. The Identification of pre-1753 Polynomials and Collections of Vascular Plants from the British Colony of Maryland. Huntia 7: 91-208.

Robinson, B. L., and M. L. Fernald. 1908. Gray's New Manual of Botany-A Handbook of the Flowering Plants and Ferns of the Central and Northeastern United State and Adjacent Canada. 7th ed. American Book Company, New York.

Rosatti, T. J. 1989. The Genera of Suborder Apocynineae (Apocynaceae and Aslepidaceae) in the Southeastern United States. J. Arnold Arbor. 70: 307-401. https://doi.org/10.5962/bhl.part.19789

Schmidt, M. F., Jr., 1993. Maryland's Geology. Tidewater Publishers, Centerville, $\mathrm{Md}$

Semple, J. C., and J. G. Chmielewski. 2006. Doellingeria. Fl. N. Amer. 20: 43-46.

Shetler, S. G., and S. S. Orli. 2000. Annotated Checklist of the Vascular Plants of the Washington-Baltimore Area. Part 1: Ferns, Fern Allies, Gymnosperms, and Dicotyledons. Department of Botany, National Museum of Natural History, Smithsonian Institution, Washington, D.C.

Shetler, S. G., and S. S. Orli. 2002. Annotated Checklist of the Vascular Plants of the Washington-Baltimore Area. Part 2: Monocotyledons. Department of Botany, National Museum of Natural History, Smithsonian Institution, Washington, D.C.

Shreve, F., M. A. Chrysler, F. H. Blodgett, and F. W. Besley. 1910. The Plant Life of Maryland. Maryland Weather Service Special Publication 3. Johns Hopkins Press, Baltimore. 
Sheviak, C. J., and P. M. Brown. 2002. Spiranthes. Fl. N. Amer. 26: 531-545. Smith, G. 2002. Schoenoplectus. Fl. N. Amer. 23: 44-60.

Smith, G., J. J. Bruhl, M. S. Gonzalez-Elizondo, and F. J. Menapace. 2002. Eleocharis. Fl. N. Amer. 23: 60-120.

Spongberg, S. A. 1972. The Genera of the Saxifragaceae in the Southeastern United States. J. Arnold Arbor. 53: 409-498.

Stieber, M. T. 1971. The Vascular Flora of Anne Arundel Co., MD: An Annotated Checklist. Castanea 36: 263-312.

Tatnall, R. R. 1946. Flora of Delaware and the Eastern Shore. Society of Natural History of Delaware, Lancaster, Pa.

Taylor, W. C., N. T. Luebke, D. M. Britton, R. J. Hickey, and D. Brunton. 1993. Isoetaceae. Fl. N. Amer. 2: 64-75.

Terrell, E. E., and P. M. Peterson. 2009. Annotated List of Maryland Grasses (Poaceae). J. Bot. Res. Inst. Texas 3: 905-919.

Terrell, E. E., J. L. Reveal, R. W. Spjut, R. F. Whitcomb, J. H. Kirkbride Jr., M. T. Cimins, and M. Strong. 2000. Annotated List of the Flora of the Beltsville Agricultural Research Center, Beltsville, Maryland. Report ARS-155. Agricultural Research Service, U.S. Department of Agriculture, Beltsville, Md. https://doi.org/10.5962/bhl.title.136933

Thiers, B. 2020. Index Herbariorum: A Global Directory of Public Herbaria and Associated Staff. New York Botanical Garden's Virtual Herbarium. http:// sweetgum.nybg.org/science/ih/ (accessed 20 June 2020).
Tucker, G. C. 1988. The Genera of Bambusoideae (Gramineae) in the Southeastern United States. J. Arnold Arbor. 69: 239-273. https://doi.org/10.5962/bhl .part. 2394

U.S. Department of Agriculture, Natural Resources Conservation Service (USDA). 2018. The PLANTS Database. https://plants.sc.egov.usda.gov/java/ (accessed 1 May 2018). National Plant Data Team, Greensboro, N.C.

Wagner, W. H., Jr., and F. S. Wagner. 1993. Botrychium. Fl. N. Amer. 2: 86-101.

Weakley, A. S. 2015. Flora of the Southern and Mid-Atlantic States. Working Draft: 21 May 2015. http://www.herbarium.unc.edu/flora.htm (accessed 1 May 2016).

Wherry, E. T. 1931. The Eastern Long-Styled Phloxes. Bartonia 13: 18-37.

Whetstone, R. D. 1983. The Sterculiaceae in the Flora of the Southeastern United States. Sida 10: 15-23.

Wiersema, J. 1997. Nelumbonaceae. Fl. N. Amer. 3: 64-77.

Williams, E., Z. Theis, and C. Hoess. 2016. Identifying a Cryptic Adiantum Population through DNA Barcoding. Amer. Fern J. 106: 135-142. https://doi .org/10.1640/0002-8444-106.2.135.1

Witman, H. W. 1954. The Flora of Cecil County, Maryland. M.S. thesis, Pennsylvania State University, University Park. 



\section{Index of Scientific Names}

The list below contains all the accepted and synonymized scientific names of flora. Entries with an asterisk $\left({ }^{*}\right)$ are synonyms. Family names are in capital letters, but names already listed in the Contents are not included in the index.

Abelmoschus esculentus, 59

Abies balsamea, 8

Abutilon, 59

"abutilon, 59

theophrasti, 59

*Acacia constricta, 49

Acalypha, 45

gracilens, 45

rhomboidea, 45

virginica, 45

*Acanthoxanthium spinosum, 26

Acer, 90, 114

campestre, 90, 114

negundo, 90

nigrum, 90

palmatum, 90

pensylvanicum, 90

platanoides, 90

pseudoplatanus, 90

rubrum, 90

"rubrum var. trilobum, 90

saccharinum, 90

*saccharum ssp. nigrum, 90

saccharum var. saccharum, 90

*saccharum var. schneckii, 90

spicatum, 90

*Acerates, 14, 99

floridana, 14, 99

longifolia, 99

viridiflora, 14

*Acetosella acetosella, 81

Achillea, 16-17, 99

"aspleniifolia, 17, 99

borealis, 16, 99

millefolium, 17, 99 *millefolium var. occidentalis, 17, 99

*occidentalis, 17, 99

ptarmica, 17

Achyranthes, 10

*aspera var. obtusifolia, 10

aspera var. pubescens, 10

*Acinos arvensis, 55, 106

*Acnida cannabina, 10

Aconitum, 83

uncinatum, 83

*uncinatum var. acutidens, 83

*uncinatum var. muticum, 83

Acorus, 9

*americanus, 9

calamus, 9

Acrocystis, 104

Actaea, 83

*alba, 83

pachypoda, 83

podocarpa, 83

racemosa, 83

*Acyrum, 53

hypericoides, 53

bypericoides var. multicaule, 53

linifolium, 53

multicaule, 53

Adiantum, 7-8, 97

aleuticum, 7, 97

capillus-veneris, 8

pedatum, 8, 97

*Adicea pumila, 93

Adlumia fungosa, 66

"Adopogon carolinianum, 21

Aegilops triuncialis, 68

Aegopodium podagraria, 12 
Aeschynomene, 46 americana, 46 virginica, 46

Aesculus, 90

flava, 90

glabra, 90

hippocastanum, 90

*octandra, 90

parviflora, 90

pavia, 90

Agalinis, 65, 109

*acuta, 65

auriculata, 65

decemloba, 65

*erecta, 65

fasciculata, 65, 109

linifolia, 65, 109

maritima var. maritima, 65

obtusifolia, 65

purpurea, 65, 109

setacea, 65

skinneriana, 65

tenuifolia, 65

Agastache, 54-55

nepetoides, 54

scrophulariifolia, 55

Agave virginica, 16, 99

Ageratina, 17

altissima var. altissima, 17

aromatica, 17

Ageratum conyzoides, 17

Agrimonia, 85

"bicknellii, 85

gryposepala, 85

microcarpa, 85

"mollis, 85

parviflora, 85

"platycarpa, 85

pubescens, 85

"pubescens var. microcarpa, 85

"pumila, 85

rostellata, 85

striata, 85

Agropyron, 73, 110, 111

"repens, 73

*subsecundum, 73, 111

"trachycaulum var. glaucum, 73, 110, 111

*trachycaulum var. unilaterale, 73, 111

Agrostemma githago, 32

Agrostis, 68, 69, 110

*alba, 68

"alba var. stolonifera, 68

"alba var. vulgaris, 68

"altissima, 68

"borealis, 68, 110

canina, 68, 110

capillaris, 68

elliottiana, 68

gigantea, 68

byemalis, 68

*intermedia, 68

mertensii, 68, 110 "palustris, 68

perennans, 68

scabra, 68

"spica-venti, 69

stolonifera, 68

"stolonifera var.

compacta, 68

stolonifera var. major, 68

"tenuis, 68

Ailanthus altissima, 91

Aira, 68, 69, 71

"caespitosa, 71

caryophyllea, 68

*caryophyllea var. capillaris, 68

elegans, 68

*elegantissima, 68

"flexuosa, 69

praecox, 68

Ajuga, 55

chamaepitys, 55

genevensis, 55

reptans, 55

Akebia quinata, 57

Albizia julibrissin, 46

Alcea, 59

"ficifolia, 59

rosea, 59

rugosa, 59

*Alchemilla microcarpa, 85, 113

Aletris, 61

aurea, 61

farinosa, 61

Alisma, 9, 98

*plantago-aquatica ssp. subcordatum, 9

subcordatum, 9

triviale, 9, 98

"Alkekengi officinarum, 92

Alliaria, 28

*officinalis, 28

petiolata, 28

*Allionia nyctaginea, 61

Allium, 12, 98

allegheniense, 12, 98

canadense var. canadense, 12, 98

cernuum, 12

sativum, 12, 98

schoenoprasum, 12

tricoccum, 12

vineale, 12

Alloteropsis cimicina, 68

Alnus, 27

glutinosa, 27

incana var. rugosa, 27

maritima ssp.

maritima, 27

"rugosa, 27

serrulata, 27

Alopecurus, 68

aequalis, 68

carolinianus, 68

geniculatus, 68 myosuroides, 68

pratensis, 68

"ramosus, 68

"Alsine, 34

media, 34

pubera, 34

Alternanthera, 10

"bettzickiana, 10

ficoidea var. bettzickiana, 10

philoxeroides, 10

pungens, 10

"repens, 10 sessilis, 10

Althaea, 59, 107-108 officinalis, 59, 107-108

*rosea, 59

Alyssum, 28

alyssoides, 28

"saxatile, 28

Amaranthus, 10

albus, 10

blitoides, 10

blitum, 10

cannabinus, 10

cruentus, 10

deflexus, 10

"graecizans var. pubescens, 10

bybridus, 10

*lividus, 10

palmeri, 10

pumilus, 10

retroflexus, 10

spinosus, 10

Ambrosia, 17

artemisiifolia, 17

*artemisiifolia var. elatior, 17

"artemisiifolia var. paniculata, 17

bidentata, 17

"glandulosa, 17

"monophylla, 17

trifida var. trifida, 17

Amelanchier, 85

arborea, 85

*arborea ssp. laevis, 85

canadensis, 85

humilis, 85

intermedia, 85

laevis, 85

nantucketensis, 85

obovalis, 85

sanguinea, 85

spicata, 85

*stolonifera, 85

Amianthium muscitoxicum, 60

Ammannia, 58

coccinea, 58

"koehnei, 58

latifolia, 58

*Ammophila, 70

arenaria, 70

breviligulata, 70
Amorpha, 46

"croceolanata, 46

*curtissii, 46

fruticosa, 46

*virgata, 46

"Ampelamus albidus, 14

Ampelopsis, 94, 116

"arborea, 94, 116

"brevipedunculata, 94

cordata, 94, 116

glandulosa, 94, 116

Amphicarpaea, 46

bracteata, 46

"bracteata var. comosa, 46

"pitcheri, 46

Amphicarpum, 68

amphicarpon, 68

"purshii, 68

Amsonia, 14, 99

"amsonia, 14, 99

tabernaemontana var. tabernaemontana, 14, 99

*Anacampseros telephioides, 36

*Anagallis, 82, 83 arvensis ssp. arvensis, 82 arvensis var. caerulea, 82 arvensis ssp. foemina, 82 minima, 83

Anaphalis, 17

margaritacea, 17

*margaritacea var. intercedens, 17

Anchistea virginica, 6

Anchusa, 27

arvensis, 27

azurea, 27

*italica, 27

Andersonglossum virginianum, 27

*Androcera rostrata, 92

Andropogon, 69, 78, 110

*argyraeus, 69

*divergens, 78

*elliottii, 69

*elliottii var. gracilior, 69

"elliottii var. projectus, 69

*furcatus, 69

gerardi, 69

"gerardii, 69

glaucopsis, 69, 110

"glomeratus var. glaucopis, 69, 110

glomeratus var. glomeratus, 69

glomeratus var. hirsutior, 69

gyrans, 69

"littoralis, 78

"pertusus, 69

*provincialis, 69

*scoparius, 78

ternarius, 69

"tetrastachyus, 69 
*virginicus var. abbreviatus, 69

*virginicus var. dealbatus, 110

*virginicus var. hirsutior, 69

virginicus var. virginicus, 69

*Aneilema keisak, 35

Anemone, 83, 113

acutiloba, 83

americana, 83

canadensis, 83

lancifolia, 83

minima, 83, 113

quinquefolia, 83

"riparia, 113

virginiana var. alba, 83, 113

virginiana var. virginiana, 83

*Anemonella thalictroides, 84

"Anemonidium canadense, 83

Anethum graveolens, 12

Angelica, 12

atropurpurea, 12

*curtisii, 12

triquinata, 12

venenosa, 12

*villosa, 12

*Anisostichus, 27

capreolata, 27

crucigera, 27

Anoda cristata, 59

Antennaria, 17

*caroliniana, 17

*fallax, 17

howellii, 17

*howellii ssp. canadensis, 17

"howellii ssp. neodioica, 17

"howellii ssp. petaloidea, 17 neglecta, 17

*neglecta var. attenuata, 17

"neodioica, 17

parlinii, 17

plantaginifolia, 17

"plantaginifolia var. arnoglossa, 17

solitaria, 17

virginica, 17

Anthemis, 17, 19

arvensis, 17

cotula, 17

*tinctoria, 19

Anthoxanthum, 69

birtum, 69

odoratum, 69

Anthriscus, 12

caucalis, 12

sylvestris, 12

*Anychia, 33

canadensis, 33

dichotoma, 33

polygonoides, 33

Apera spica-venti, 69

Aphanes, 85, 113

australis, 85, 113

microcarpa, 85, 113

Aphyllon uniflorum, 65
Apios, 46, 105

americana, 46

*apios, 46

priceana, 46, 105

Apium, 12, 13

graveolens, 12

"leptophyllum, 13

Aplectrum, 63

byemale, 63

"spicatum, 63

Apluda mutica, 69

Apocynum, 14

androsaemifolium, 14

cannabinum, 14

*sibiricum, 14

Aquilegia, 83

canadensis, 83

vulgaris, 83

Arabidopsis, 28

lyrata lyrate, 28

thaliana, 28

Arabis, 28, 29, 30, 31

*canadensis, 29

"glabra, 31

" hirsuta var. adpressipilis, 28

*hirsuta var.

pycnocarpa, 28

*laevigata, 29

*laevigata var. burkii, 29

"lyrata, 28

*missouriensis, 29

patens, 28

"perstellata var. shortii, 29

pycnocarpa var. adpressipilis, 28

pycnocarpa var. pycnocarpa, 28

*shortii, 29

*virginica, 30

Arachis hypogaea, 46

Aralia, 15

elata, 15

hispida, 15

nudicaulis, 15

racemosa, 15

spinosa, 15

Arctium, 17

lappa, 17

minus, 17

tomentosum, 17

Arctostaphylos uva-ursi, 44

Arenaria, 32, 33

*caroliniana, 33

leptoclados, 32

*michauxii, 33

serpyllifolia, 32

*stricta, 33

Arethusa bulbosa, 63

Argemone, 66

*alba, 66

albiflora, 66

"leiocarpa, 66

mexicana, 66

*Argentacer saccharinum, 90
Argentina anserina, 85, 113

Arisaema, 15

*atrorubens, 15

dracontium, 15

pusillum, 15

stewardsonii, 15

triphyllum, 15

"triphyllum ssp. pusillum, 15

"triphyllum ssp. stewardsonii, 15

Aristida, 69

adscensionis, 69

"curtissii, 69

dichotoma var. curtissii, 69

dichotoma var. dichotoma, 69

"gracilis, 69

*intermedia, 69

*lanata, 69

lanosa, 69

longespica var. geniculata, 69

longespica var. longespica, 69

oligantha, 69

purpurascens, 69

*purpurascens var. virgata, 69

tuberculosa, 69

virgata, 69

Aristolochia, 16

clematitis, 16

"durior, 16

*macrophylla, 16

"serpentaria, 16

Armoracia, 28, 30

*aquatica, 30

*lacustris, 30

"lapathifolia, 28

rusticana, 28

Arnica acaulis, 17

Arnoglossum, 17 atriplicifolium, 17

*mueblenbergii, 17

reniforme, 17

Aronia, 85

arbutifolia, 85

"arbutifolia var. atropurpurea, 85

"atropurpurea, 85

melanocarpa, 85

prunifolia, 85

Arrhenatherum, 69

elatius var. bulbosum, 69

elatius var. elatius, 69

*Arsenococcus, 44

frondosus, 44

ligustrinus, 44

Artemisia, 17, 99

abrotanum, 17

absinthium, 17

annua, 17

biennis, 17, 99

ludoviciana, 17 pontica, 17, 99

scoparia, 17

stelleriana, 17

vulgaris, 17

Arthraxon, 69

*ciliaris var. cryptatherus, 69

hispidus, 69

"hispidus var. cryptatherus, 69

Arum italicum italicum, 15

Aruncus, 85

*allegheniensis, 85

dioicus, 85

Arundinaria, 69, 110

gigantea, 69, 110

*gigantea ssp. tecta, 69

tecta, 69, 110

Arundo donax, 69

Asarum, 16

canadense, 16

reflexum, 16

virginicum, 16

Asclepias, 14, 99

amplexicaulis, 14

exaltata, 14

incarnata ssp. incarnata, 14

incarnata ssp. pulchra, 14

lanceolata, 14

linearis, 14, 99

longifolia, 14, 99

*obtusifolia, 14

"phytolaccoides, 14

purpurascens, 14

quadrifolia, 14

rubra, 14

syriaca, 14

tuberosa ssp. tuberosa, 14

variegata, 14

verticillata, 14, 99

viridiflora, 14

*Ascyrum stans, 52

Asimina triloba, 12

Asparagus officinalis, 16

Asperugo procumbens, 27,101

*Asperula, 88, 89, 114

arvensis, 88 odorata, 89,114

Asplenium, 5-6

"acrostichoides, 6

*angustifolium, 6

bradleyi, 5

*cryptolepis, 6

"filix-femina ssp. asplenioides, 6

*filix-femina var. asplenioides, 6

montanum, 5

"parvulum, 5

pinnatifidum, 5

platyneuron, 5

"platyneuron var. bacculum-rubrum, 5

resiliens, 5 
Asplenium (continued) rhizophyllum, 5

ruta-muraria, 6

"ruta-muraria var. cryptolepis, 6

scolopendrium var americanum, 6

trichomanes trichomanes, 6

*Aspris caryophyllea, 68

Aster, 17, 19, 20, 21, 22, $24,25,26,99$

*acuminatus, 22

"asperifolius, 26

*amptosorus, 25

"concinnus, 25

*concolor, 25

*cordifolius, 25

*corrigiatus, 26

*depauperatus, 25

"divaricatus, 20

*drummondii, 25

*dumosus, 25

*dumosus var. coridifolius, 25

*ericoides, 25

*falcidens, 25

"gracilis, 20

"hirsuticaulis, 25

*infirmus, 19

"laevis, 25

"laevis var. amplifoius, 25

"laevis var. concinnus, 25

*lanceolatus, 25

"lanceolatus var. latifolium, 25

"linariifolius, 21

*lowrieanum, 25

"macrophyllus, 20

"mohrii, 26

"multiflorus, 25

"nemoralis, 22

*novae-angliae, 25

"novi-belgii, 25

"novi-belgii var. elodes, 25

*oblongifolius, 25

"patens, 25

*patens var. gracilis, 25

"patens var. tenuicaulis, 25

"paternus, 24

"phlogifolius, 25

"pilosus, 25

"pilosus var. pringlei, 25

"plumosus, 25

"praealtus, 25

*prenanthoides, 25

*proteus, 26

"puniceus var. scabricaulis, 25

"racemosus, 25

"radula, 20

*sagittifolius, 26

"schreberi, 20

*sericocarpoides, 99

"shortii, 25 *simplex var. ramosissimus, 25

"solidagineus, 24

"spectabilis, 20

*steeleorum, 25

*subulatus, 25

*sylvestris, 26

tataricus, 17

"tenuicaulis, 25

"tenuifolius, 25

*triangularis, 26

"truellius, 26

"umbellatus, 19

*undulatus, 26

"undulatus var. diversifolius, 26

"undulatus var. loriformis, 26

"urophyllus, 26

*vimineus var. foliolosus, 25

Astilbe biternata, 91, 115

Astragalus, 46

canadensis var. canadensis, 46

"carolinianus, 46

distortus, 46

"halei, 46

hamosus, 46

Athyrium, 6

angustum, 6

asplenioides, 6

"filix-femina ssp. angustum, 6

*filix-femina var. angustum, 6

*filix-femina var. michauxii, 6

"pycnocarpon, 6

*thelypterioides, 6

Atriplex, 10

*arenaria, 10

"cristata, 10

elegans var. elegans, 10

"hastata, 10

hortensis, 10

littoralis, 10

mucronata, 10

"nitens, 10

patula, 10

"patula var. japonica, 10

prostrata, 10

rosea, 10

"thornberi, 10

Aureolaria, 65

*dispersa, 65

flava, 65

laevigata, 65

"microcarpa, 65

pedicularia, 65

virginica, 65

Aurinia saxatilis, 28

Avena, 69

fatua, 69

sativa, 69
Avenella flexuosa, 69

Axonopus furcatus, 69

"Azalea, 44, 45, 104

arborescens, 44

atlantica, 45

calendulacea, 45, 104

nudiflora, 45

prinophylla, 45

serrulata, 45

viscosa, 45

viscosa var. glauca, 45

Azolla caroliniana, 8

Baccharis halimifolia, 17

Bacopa, 66-67, 109

caroliniana, 66, 109

*cyclophylla, 67

innominata, 67

monnieri, 67

rotundifolia, 67

*stragula, 67

Babia schaffneri, 17

Ballota nigra, 55

Baptisia, 46

australis, 46

tinctoria, 46

Barbarea, 29

verna, 29

vulgaris, 29

Bartonia, 50

"lanceolata, 50

paniculata ssp. paniculata, 50 virginica, 50

Bassia, 10-11

hirsuta, 10

scoparia, 11

*Batschia canescens, 28

"Belamcanda chinensis, 53

Bellis perennis, 17

Berberis, 26-27, 101

"bealei, 27

canadensis, 26, 101

julianae, 26

thunbergii, 27, 101

vulgaris, 27

Berchemia scandens, 85, 113

Berteroa incana, 29

Beta vulgaris vulgaris, 11

Betula, 27, 101

*alba, 27

alleghaniensis, 27

lenta, 27

*lutea, 27

nigra, 27

papyrifera, 27, 101

populifolia, 27

pubescens, 27

*Bicuculla, 66

cucullaria, 66

eximia, 66

Bidens, 17-18, 99

aristosa, 17

beckii, 17 bidentoides, 17

*bidentoides var. mariana, 17

bipinnata, 17

cernua, 17

comosa, 17

connata, 17

*oronata, 18

discoidea, 17

frondosa, 17

*involucrata, 17

laevis, 18

*leucantha, 18

mitis, 18, 99

"nashii, 18

pilosa, 18

polylepis, 18

tenuisecta, 18

trichosperma, 18, 99

vulgata, 18

Bifora testiculata, 12

Bignonia, 27

capreolata, 27

"radicans, 27

*Blastania fimbristipula, 36

"Blephariglottis, 64

ciliaris, 64

cristata, 64

lacera, 64

peramoena, 64

Blephilia, 55

ciliata, 55

hirsuta, 55

Bletilla striata, 63

Boechera, 29

burkii, 29

canadensis, 29

dentata, 29

laevigata, 29

missouriensis, 29

*shortii, 29

Boehmeria, 93, 115

cylindrica, 93

*drummondiana, 93

nivea, 93, 115

Boerhavia, 61

coccinea, 61

erecta, 61

Bolboschoenus, 37, 103

fluviatilis, 37, 103

maritimus ssp. paludosus, 37, 103

novae-angliae, 37

robustus, 37

Boltonia, 18

asteroides var. asteroides, 18 asteroides var. glastifolia, 18

Borago officinalis, 27, 101

"Borodinia, 29

burkii, 29

canadensis, 29

dentata, 29

laevigata, 29

missouriensis, 29 
Borrichia frutescens, 18

Bothriochloa pertusa, 69

Botrychium, 7, 97

angustisegmentum, 7

*dissectum var. obliquum, 7

*jenmanii, 7, 97

*lanceolatum ssp. angustisegmentum, 7

matricariifolium, 7

*multitifidum, 7

*obliquum, 7

simplex, 7

*virginianum, 7

Botrypus virginianus, 7

Bouteloua, 69, 110

aristidoides, 69

barbata, 69

curtipendula, 69

hirsuta, 69, 110

"Brachiaria, 75, 79

distichophylla, 79

eruciformis, 75

plantaginea, 79

platyphylla, 79

ramosa, 79

reptans, 79

subquadripara, 79

texana, 79

Brachyelytrum, 70

aristosum, 70

erectum, 70

*erectum var. septentrionale, 70

*septentrionale, 70

"Bramia monnieri, 67

Brasenia, 31

"purpurea, 31

schreberi, 31

Brassica, 29, 30

"alba, 30

*campestris, 29

"erucastrum, 30

"hirta, 30

"japonica, 29

juncea, 29

*kaber, 30

napus, 29

nigra, 29

oleracea, 29

rapa, 29

*Brauneria, 19

pallida, 19

purpurea, 19

Brickellia eupatorioides var. eupatorioides, 18

Briza, 70, 110

media, 70, 110

minor, 70

Bromus, 70, 110

"altissimus, 70

arvensis, 70

briziformis, 70

catharticus, 70

ciliatus, 70 commutatus, 70

diandrus, 70

*dudleyi, 70

hordeaceus, 70

*incanus, 70

inermis, 70

japonicus, 70

kalmii, 70

"laeviglumis, 70

latiglumis, 70

madritensis, 70, 110

"mollis, 70

nottowayanus, 70

pubescens, 70

"purgans, 70

"purgans var. incanus, 70

racemosus, 70

"rigidus, 70

rubens, 70,110

secalinus, 70

sterilis, 70

tectorum, 70

*unioloides, 70

Broussonetia papyrifera, 61

Brunnera macrophylla, 27

Bryodesma rupestre, 5

Buchnera americana, 65

Buddleja davidii, 91

Buglossoides arvensis, 27

Bulbostylis, 37

capillaris, 37

*capillaris var. isopoda, 37

*hirta, 37

hispidula, 37

*Bumelia lycioides, 90, 115

Bupleurum, 12

lancifolium, 12

odontites, 12

"protractum, 12

rotundifolium, 12

*Bursa bursa-pastoris, 29

*Butneria fertilis, 31

"Buxella brachycera, 44

Cabomba, 31, 113

carolinensis, 113

caroliniana, 31

*Cacalia, 17, 23

atriplicifolia, 17

muehlenbergii, 17

suaveolens, 23

Cajanus, 46

cajan, 46

scarabaeoides, 46

Cakile, 29

"cakile, 29

edentula, 29

maritima, 29

Calamagrostis, 70, 74

arenaria, 70

breviligulata ssp. breviligulata, 70

canadensis var. canadensis, 70 "cinnoides, 74

"coarctata, 74

porteri, 70

Calepina irregularis, 29

Calibrachoa parviflora, 91

Calla palustris, 15

Callicarpa, 55

americana, 55

dichotoma, 55

*Callisia, 35, 103

graminea, 35, 103

rosea, 35,103

Callitriche, 67

"austinii, 67

*deflexa, 67

heterophylla, 67

palustris, 67

stagnalis, 67

terrestris, 67

Calopogon, 63

"pulchellus, 63

tuberosus var. tuberosus, 63

Caltha palustris, 83

Calycanthus, 31

*fertilis, 31

floridus, 31

*floridus var. glaucus, 31

*floridus var. laevigatus, 31

"nanus, 31

Calystegia, 35

pubescens, 35

sepium, 35

"sepium ssp. americana, 35

*sepium ssp. angulata, 35

*sepium ssp. appalachiana, 35

silvatica ssp. fraterniflora, 35

"spithamaea ssp. purshiana, 35

spithamaea ssp. spithamaea, 35 spithamaea ssp. stans, 35

Camassia scilloides, 16

Camelina, 29

microcarpa, 29

sativa, 29

"sativa ssp. microcarpa, 29

Campanula, 31, 101

americana, 31

"aparinoides, 31

divaricata, 31, 101

rapunculoides, 31

rotundifolia, 31

*Campanulastrum americanum, 31

*Campe, 29

barbarea, 29

stricta, 29

verna, 29

Campsis radicans, 27

"Camptosorus rhizophyllus, 5

Cannabis sativa, 31
"Cantharospermum scarabaeoides, 46

Capnoides, 66

*flavulum, 66

sempervirens, 66

"Capriola dactylon, 71

Capsella bursa-pastoris, 29

Capsicum, 91

annuum, 91

*annuum var. glabriusculum, 91

"baccatum, 91

"frutescens, 91

Caragana arborescens, 46

*Carara didyma, 30

Cardamine, 29, 30, 101

angustata, 29

*arenicola, 29

bulbosa, 29

concatenata, 29

diphylla, 29

douglassii, 29

flexuosa, 29, 101

hirsuta, 29

impatiens, 29

longii, 29

occulta, 29, 101

parviflora var. arenicola, 29

pensylvanica, 29

pratensis var. palustris, 29,101

*rhomboidea, 29

rotundifolia, 29

*virginica, 30

*Cardaria draba, 30

Cardiocrinum cordatum, 57

Carduus, 18

acanthoides, 18

*altissimus, 18

*arvense var. horridum, 18

*arvensis, 18

crispus, 18

*discolor, 18

*lanceolatus, 18

*muticus, 18

nutans ssp. macrolepis, 18

*spinosissimus, 18

"spinosissimus var. elliottii, 18

Carex, 37-40, 103-104

abscondita, 37

acutiformis, 37

aestivalis, 37

aggregata, 37, 103

alata, 37

albicans var. albicans, 37

albicans var. emmonsii, 37

albolutescens, 37

albursina, 37

*allegheniensis, 38

amphibola, 37

"amphibola var. rigida, 39

*amphibola var. turgida, 38

*angustior, 38

annectens, 37 


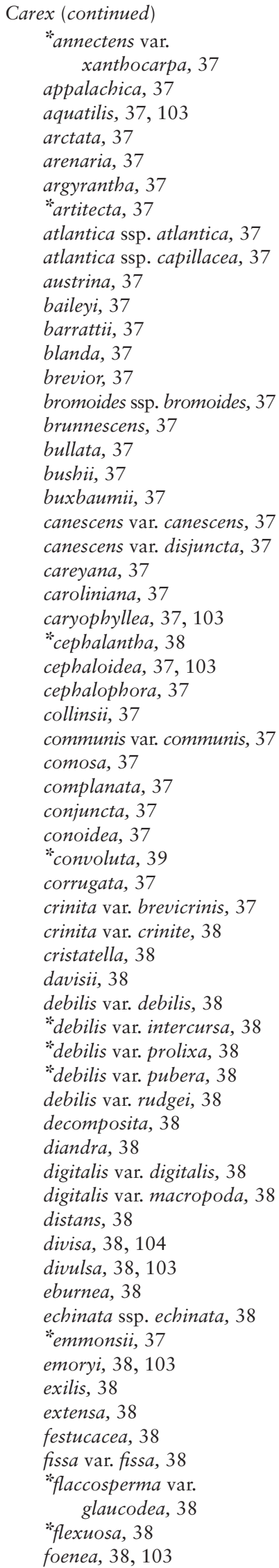

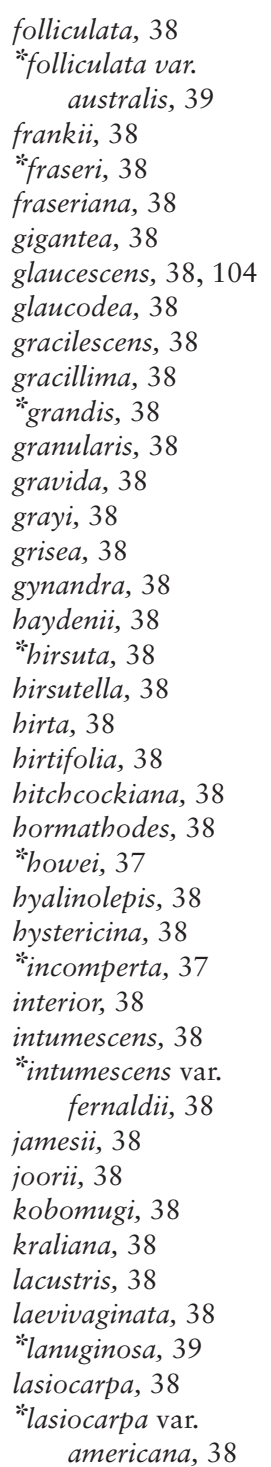

laxiculmis var. copulata, 38 laxiculmis var. laxiculmis, 38 laxiflora, 39

"laxiflora var. patulifolia, 39

*laxiflora var. serrulata, 39

leavenworthii, 39

leptalea var. harperi, 39

leptalea var. leptalea, 39

leptonervia, 39

lonchocarpa, 39

longii, 39

louisianica, 39

lucorum, 39

lupuliformis, 39

lupulina, 39

lurida, 39

meadii, 39

mesochorea, 39

michauxiana, 39

mitchelliana, 39

molesta, 39

molestiformis, 39

mublenbergii var. enervis, 39 mublenbergii var. mublenbergii, 39

* mublenbergii var. xalapensis, 39

muricata, 39

nigromarginata, 39

normalis, 39

novae-angliae, 39, 103

*oblita, 40

oklahomensis, 39

oligocarpa, 39

oxylepis, 39

"pairaei, 39

pallescens, 39, 103

pedunculata, 39

pellita, 39

pensylvanica, 39

"plana, 39

planispicata, 39

plantaginea, 39

platyphylla, 39

polymorpha, 39

prasina, 39

projecta, 39

radiata, 39

retroflexa, 39

retrorsa, 39, 104

reznicekii, 39

richardsonii, 39

rosea, 39

"rosea var. radiata, 39

rostrata, 39, 104

"rostrata var. utriculata, 40

sartwellii, 39, 104

scabrata, 39

scoparia, 39

seorsa, 39

shortiana, 39

*siccata, 38, 103

silicea, 39

"smalliana, 39

sparaginoides, 103

sparganioides, 40

spicata, 40

squarrosa, 40

stipata var. maxima, 40

stipata var. stipata, 40

*stipata var. uberior, 40

straminea, 40

striata, 40

striatula, 40

stricta, 40

"strictior, 40

styloflexa, 40

swanii, 40

tenera, 40

tenuiflora, 40, 104

tetanica var. canbyi, 40

texensis, 40

tonsa var. rugosperma, 40

tonsa var. tonsa, 40

torta, 40

tribuloides var. tribuloides, 40

"triceps, 38

trichocarpa, 40 trisperma, 40

tuckermanii, 40

typhina, 40

*uberior, 40

umbellata, 40

utriculata, 40, 104

venusta, 40

*venusta var. minor, 40

verrucosa, 40, 104

vesicaria, 40

vestita, 40

virescens, 40

viridula, 40, 104

vulpinoidea, 40

"walteriana, 40

willdenowii, 40

woodii, 40

Carpinus, 27

caroliniana, 27

"caroliniana var. virginiana, 27

*caroliniana ssp. virginiana, 27

"Carthamus tinctorius, 18

Carum carvi, 12

Carya, 53-54

*alba, 54

cordiformis, 53

glabra, 53

illinoinensis, 53

laciniosa, 53

ovalis, 53

ovata, 53

pallida, 53

tomentosa, 54

*Cassia, 46, 47, 49, 105

baubinioides, 49

fasciculata, 46

hebecarpa, 49

marilandica, 49

mississippiensis, 46

nictitans var. mohrii, 47

obovata, 49

obtusifolia, 49

occidentalis, 49, 105

torta, 49

*Castalia, 61

lekophylla, 61

minor, 61

odorata, 61

odorata var. rosea, 61

Castanea, 50

*ashei, 50

dentata, 50

pumila, 50

Castilleja coccinea, 65

Catalpa, 27

bignonioides, 27

*atalpa, 27

ovata, 27

speciosa, 27

*Cathartolinum, 58

intercursum, 58

medium, 58

striatum, 58 
sulcatum, 58 virginianum, 58

*Caucalis nodosa, 14

Caulophyllum, 27

giganteum, 27

thalictroides, 27

Causonis japonica, 94

"Cayratia japonica, 94

Ceanothus americanus, 85

Celastrus, 34

orbiculatus, 34

scandens, 34

Celosia, 11

argentea, 11

"margaritacea, 11

Celtis, 31, 102

*georgiana, 31

laevigata, 31, 102

"mississippiensis, 31, 102

occidentalis, 31, 102

*occidentalis var. georgiana, 31

*occidentalis var. pumila, 31

"pumila, 31

*smallii, 31

tenuiflora, 102

tenuifolia, 31

Cenchrus, 70, 110

"americanus, 70

"carolinianus, 70

*compressus, 70

echinatus, 70

*incertus, 70, 110

longispinus, 70

"macrocephalus, 70

"pauciflorus, 70, 110

purpurascens, 70

spicatus, 70

spinifex, 70, 110

tribuloides, 70

Centaurea, 18, 99

benedicta, 18

calcitrapa, 18

cineraria, 18, 99

cyanus, 18

depressa, 18

*dubia, 18

jacea, 18

*maculosa, 18

nigra, 18

nigrescens, 18

scabiosa, 18, 99

solstitialis, 18

stoebe ssp. micranthos, 18

*vochinensis, 18

Centaurium, 50, 51

erythraea, 50

pulchellum, 50

"spicatum, 51

*umbellatum, 50

Centella, 12

asiatica, 12

*erecta, 12

Centrosema virginianum, 46
${ }^{*}$ Centrostachys, 10

aspera, 10

indica, 10

*Centunculus minimus, 83

Cephalanthus occidentalis, 88

Cerastium, 32-33

arvense ssp. arvense, 32

arvense ssp. strictum, 32

"arvense var. villosum, 33

brachypetalum, 32

fontanum ssp. vulgare, 32

glomeratum, 32

"longepedunculatum, 32

nutans var. nutans, 32

pumilum, 32

semidecandrum, 32

velutinum var.

velutinum, 32

velutinum var. villosissimum, 33

*viscosum, 32

*vulgatum, 32

Ceratophyllum, 34

demersum, 34

echinatum, 34

Cercis canadensis, 46

Chaenomeles, 85

japonica, 85

speciosa, 85

Chaenorhinum minus minus, 67

Chaerophyllum, 12

*floridanum, 12

procumbens var. procumbens, 12

procumbens var. shortii, 12 tainturieri, 12

Chaiturus marrubiastrum, 55

Chamaecrista, 46-47

"aspera var. mohrii, 47

"brachiata, 46

"depressa, 46

fasciculata, 46

*fasciculata var. macrosperma, 46

*littoralis, 46

"mississippiensis, 46

"mohrii, 47

*multipinnata, 47

nictitans var. nictitans, 47

*procumbens, 47

"robusta, 46

Chamaecyparis thyoides, 8

Chamaedaphne calyculata, 44

Chamaelirium luteum, 60

Chamaenerion angustifolium, 62

*Chamaepericlymenum canadense, 36

*Chamaesyce, 45, 46

birta, 45

humistrata, 46

hypericifolia, 46

hyssopifolia, 46

maculata, 46

nutans, 46

polygonifolia, 46 prostrata, 46

tracyi, 46

vermiculata, 46

*Chamerion angustifolium, 62

Chasmanthium, 70

latifolium, 70

laxum, 70

*Cheilanthes lanosa, 8

*Cheirina aspera, 30, 101

"Cheirinia cheiranthoides, 30

Chelidonium majus, 66

Chelone, 67

glabra, 67

obliqua, 67

Chenopodiastrum, 11

murale, 11

simplex, 11

Chenopodium, 11, 98

album, 11

"album var. missouriense, 11

*amaranticolor, 11

*ambrosioides, 11

berlandieri, 11

*berlandieri var. boscianum, 11

*berlandieri var. bushianum, 11

*berlandieri var. macrocalycium, 11

bonus-henricus, 11

"botrys, 11

"bushianum, 11

carnosulum, 11

*desiccatum var. leptophylloides, 11

*farinosum, 11

*giganteum, 11

"gigantospermum, 11

glaucum var. glaucum, 11

"hybridum simplex, 11

*lanceolatum, 11

leptophyllum, 11

macrospermum, 11

"missouriense, 11

"murale, 11

opulifolium, 11

*paganum, 11

*plantaginellum, 11

polyspermum, 11

"polyspermum var. acutifolium, 11

*polyspermum var. obtusifolium, 11

pratericola, 11

rubrum, 11, 98

standleyanum, 11

urbicum, 11, 98

"viride, 11

vulvaria, 11

Chimaphila, 44

maculata, 44

umbellata, 44

"umbellata var.

cisatlantica, 44
Chionanthus virginicus, 61

Chloris, 70

verticillata, 70

virgata, 70

Chondrilla juncea, 18

Chrozophora tinctoria, 45

"Chrysanthemum, 20, 22, 26, 100

balsamita, 26

leucanthemum, 22

parthenium, 26

segetum, 20, 100

Chrysogonum, 18

"australe, 18

virginianum var. virginianum, 18

Chrysopsis, 18, 23

"graminifolia var. latifolia, 23

mariana, 18

Chrysosplenium americanum, 91

Cicer arietinum, 47

Cichorium intybus, 18

Cicuta, 13

bulbifera, 13

*curtissii, 13

* maculata var. bolanderi, 13

maculata var. maculate, 13

Cimicifuga, 83

*americana, 83

*racemosa, 83

Cinna, 70

arundinacea, 70

latifolia, 70

Circaea, 62

alpina, 62

canadensis ssp. canadensis, 62

"latifolia, 62

*lutetiana, 62

"quadrisulcata, 62

Cirsium, 18

altissimum, 18

arvense, 18

discolor, 18

horridulum var. horridulum, 18

muticum, 18

pumilum, 18

smallii, 18

vulgare, 18

Citrullus, 36

"citrullus, 36

lanatus var. lanatus, 36

*vulgaris, 36

*Citrus trifoliata, 89

Cladium mariscoides, 40

Claytonia, 61

caroliniana, 61

"media, 61

virginica, 61

*virginica var. acutiflora, 61 
Claytosmunda claytoniana, 7

${ }^{*}$ Cleistes divaricata, 63

Cleistesiopsis divaricata, 63

Clematis, 83

"dioscoreifolia, 83

occidentalis var. occidentalis, 83

ochroleuca, 83

"paniculata, 83

terniflora, 83

*verticillaris, 83

viorna, 83

virginiana, 83

vitalba, 83

Cleome, 35

hassleriana, 35

"houtteana, 35

ornithopodioides, 35

*spinosa, 35

viscosa, 35

Clerodendrum japonicum, 55, 106

Clethra alnifolia, 35

Clinopodium, 55, 106

acinos, 55, 106

calamintha, 55

*calamintha var. nepetoides, 55

*nepeta, 55

*nepeta ssp. glandulosa, 55 vulgare, 55

Clintonia, 57

alleghaniensis, 57

borealis, 57

umbellulata, 57

Clitoria mariana, 47

Cnicus benedictus, 18

"Coeloglossum viride, 63

*Coelorachis rugosa, 75

Coincya monensis, 29

Colchicum autumnale, 35, 103

Coleataenia, 70-71, 110

anceps ssp. anceps, 70

anceps ssp. rhizomata,

70, 110

longifolia ssp. combsii, 70

"longifolia ssp. elongata, 71

longifolia ssp. longifolia, 71

rigidula ssp. condensa, 71

rigidula ssp. rigidula, 71

stipitata, 71

Collinsonia, 55

canadensis, 55

*scabriuscula, 55

"tuberosa, 55

Comandra umbellata, 90

Commelina, 35

*angustifolia, 35

caroliniana, 35

communis, 35

*communis var. ludens, 35

"crispa, 35

diffusa, 35 erecta, 35

*erecta var. angustifolia, 35

"hirtella, 35

*longicaulis, 35

virginica, 35

*COMPOSITAE, 99-101

Comptonia peregrina, 61

Conium maculatum, 13

Conoclinium coelestinum, 18

Conopholis americana, 65

Conringia orientalis, 29

Consolida, 83, 113

ajacis, 83

*ambigua, 83

regalis, 83, 113

Convallaria majalis, 16

Convolvulus, 35, 103

arvensis, 35

"incanus, 35

"nashii, 35

"purshianus, 35

*sepium, 35

"sepium var.

fraterniflorus, 35

"spithamaeus, 35

wallichianus, 35, 103

Conyza, 18

canadensis var. canadensis, 18 canadensis var. pusilla, 18

Coptis, 83, 84

trifolia, 84

trifolia var. groenlandica, 83

Corallorhiza, 63

maculata, 63

"micrantha, 63

odontorhiza, 63

trifida, 63

wisteriana, 63

Coreopsis, 18-19

"cardaminefolia, 19

*crassifolia, 18

grandiflora, 18

"grandiflora var.

harveyana, 18

"grandiflora var. saxicola, 18

lanceolata, 18

rosea, 19

*saxicola, 18

tinctoria, 19

tripteris, 19

verticillata, 19

Coriandrum sativum, 13

"Coridochloa cimicina, 68

Cornus, 36

"alba, 36

alternifolia, 36

amomum, 36

canadensis, 36

florida, 36

"foemina, 36

obliqua, 36

racemosa, 36 rugosa, 36

*sericea, 36

stolonifera, 36

stricta, 36

*Coronilla, 49

cretica, 49

varia, 49

*Coronopus didymus, 30

Corrigiola litoralis, 33, 102

Corydalis, 66, 109

aurea, 66, 109

flavula, 66

incisa, 66

*sempervirens, 66

Corylus, 27

americana, 27

cornuta var. cornuta, 27

"rostrata, 27

*Corynandra viscosa, 35

Cosmos, 19

parviflorus, 19

sulphureus, 19

Cota tinctoria, 19

Cotinus coggygria, 12, 98

Crassula aquatica, 36

Crataegus, 85-86, 113-114

*alnorum, 86, 113

"arborea, 86

"armentalis, 86

"basilica, 86, 113

"biltmoreana, 86

*blanda, 86

*boyntonii, 86

calpodendron, 85

"canbyi, 86

chrysocarpa, 85

coccinea var. coccinea, 86

"contrita, 86

crus-galli var. crus-galli, 86

*crus-galli var. pyacanthifolia, 86

dodgei, 86

"evansiana, 85

*ferrissii, 86, 113

*fabellata, 86

*gattingeri, 86

*inanis, 86

intricata, 86

*iracunda var. populnea, 86,113

*lanceolata, 86

macracantha, 86, 113

macrosperma, 86

margarettae, 86, 113

"mendosa, 86

*mercerensis, 85

"mohrii, 86

monogyna var. monogyna, 86

*pedicellata, 86

"penita, 86

*pennsylvanica, 86

phaenopyrum, 86

"pinetorum, 86 "populifolia, 86 populnea, 86, 113

pruinosa, 86

"pruinosa var. dissona, 86

punctata, 86

"punctata var. aurea, 86

"punctata var. canescens, 86

"punctata var. microphylla, 86

rubella, 86

*rugosa, 86

*sargentii, 86

schuettei var. schuettei, 86, 113-114

*sicca, 85

*signata, 86

*silvicola, 86, 113

*stolonifera, 86, 113

succulenta var. succulenta, 86

*tatnalliana, 86

*tomentosa, 85

*tortilis, 86, 113

uniflora, 86

viridis var. viridis, 86

*vulsa, 86

Crepidomanes intricatum, 7

Crepis, 19, 26

capillaris, 19

*japonica, 26

pulchra, 19

tectorum, 19

Crocanthemum, 34

bicknellii, 34

canadense, 34

propinquum, 34

Crocus vernus, 53

Crotalaria, 47, 105

"angulata, 47, 105

*maritima, 47, 105

pumila, 47

rotundifolia, 47, 105

sagittalis, 47

Croton, 45, 105

bonplandianus, 45

capitatus var. capitatus, 45

glandulosus var. septentrionalis, 45

michauxii, 45, 105

monanthogynus, 45 willdenowii, 45, 105

*Crotonopsis, 45, 105

elliptica, 45

linearis, 45, 105

*Cruciata pedemontana, 89

*CRUCIFERAE, 101

Cryptotaenia canadensis, 13

Ctenolepis cerasiformis, 36

Cubelium concolor, 94

Cunila origanoides, 55

Cuphea, 58

"petiolata, 58

viscosissima, 58 
Cuscuta, 35-36, 103

*arvensis, 35

campestris, 35

cephalanthi, 35, 103

compacta var. compacta, 35

coryli, 35

cuspidata, 35

epilinum, 35, 103

epithymum, 35, 103

gronovii, 36

"gronovii var. latiflora, 36

indecora var. indecora, 36

*indecora var. neuropetala, 36

"pentagona, 35

polygonorum, 36

rostrata, 36

suaveolens, 36

Cuthbertia, 35, 103

graminea, 35, 103

rosea, 35, 103

Cycloloma atriplicifolium, 11

Cyclospermum leptophyllum, 13

Cydonia oblonga, 86

Cymbalaria muralis, 67

Cymophora accedens, 19

*Cymophyllus, 38

fraseri, 38

fraserianus, 38

Cynanchum, 14

laeve, 14

"louiseae, 14

Cynodon dactylon, 71

Cynoglossum, 27

officinale, 27

*virginianum, 27

Cynosurus, 71

cristatus, 71

echinatus, 71

*Cynoxylon floridum, 36

*Cynthia, 21, 100

dandelion, 21

virginica, 100

Cyperus, 40-41, 104

acuminatus, 40

*aristatus, 41

bipartitus, 40

brevifolioides, 40

compressus, 40

croceus, 40

*cylindricus, 41

dentatus, 40

diandrus, 40

difformis, 40

"dipsaciformis, 41

echinatus, 40

engelmannii, 40, 104

erythrorbizos, 40

esculentus var.

leptostachyus, 40

*esculentus var. sativus, 40

filicinus, 40

filiculmis, 40 flavescens, 40

flavicomus, 40

fuscus, 40

"globulosus, 40

grayi, 40

"halei, 40

haspan, 40

hortensis, 41

houghtonii, 41

bystricinus, 41

*inflexus, 41

iria, 41

lancastriensis, 41

lupulinus ssp. lupulinus, 41

lupulinus ssp. macilentus, 41

*microdontus, 41

microiria, 41

"multiflorus, 40

"nashii, 41

"nuttallii, 40

odoratus, 41

*ovularis, 40

"paniculatus, 41

plukenetii, 41

polystachyos, 41

*polystachyos var. texensis, 41

pseudovegetus, 41

refractus, 41

retrofractus, 41

retrorsus, 41

"retrorsus var. cylindricus, 41

"retrorsus var. nashii, 41

"retrorsus var. robustus, 40

"rivularis, 40

rotundus, 41

squarrosus, 41

"stenolepis, 41

strigosus, 41

"strigosus var. robustior, 41

subsquarrosus, 41

"tenuifolius, 41

*torreyi, 41

Cypripedium, 3, 63

acaule, 63

*calceolus var. pubescens, 63

candidum, 3, 63

"hirsutum, 63

parviflorum var. parviflorum, 63

*parviflorum var. pubescens, 63

"pubescens, 63

reginae, 63

Cyrtomium fortunei, 6

Cystopteris, 6

bulbifera, 6

"fragilis var. protrusa, 6

protrusa, 6 tennesseensis, 6

tenuis, 6

Cytisus scoparius, 47

Dactylis glomerata, 71

Dactyloctenium, 71

aegyptium, 71

germinatum, 71

Dactylorhiza viridis var. virescens, 63

Dalea, 47

brachystachya, 47

carthagenensis, 47

"lemmonii, 47

Dalibarda repens, 86

Danthonia, 71

compressa, 71

sericea, 71

spicata, 71

*Dasistoma, 65

flava, 65

laevigata, 65

virginica, 65

Dasyochloa pulchella, 71

"Dasystephana, 51

latifolia, 51

parvifolia, 51

puberula, 51

saponaria, 51

villosa, 51

Datura, 91

*ferox, 91

inoxia, 91

*meteloides, 91

quercifolia, 91

stramonium, 91

"stramonium var. tatula, 91

wrightii, 91

Daucus carota, 13

"Decachaena, 44

baccata, 44

frondosa, 44

Decodon verticillatus, 58

Delphinium, 83, 113

"ajacis, 83

"consolida, 83, 113

exaltatum, 83

tricorne, 83

*urceolatum, 83

Dendrolycopodium, 5

dendroideum, 5

bickeyi, 5

obscurum, 5

"Dendropogon usneoides, 31

Dennstaedtia punctilobula, 6

"Dentaria, 29

heterophylla, 29

laciniata, 29

Deparia acrostichoides, 6

"Deringa canadensis, 13

Deschampsia, 69, 71

caespitosa glauca, 71

*flexuosa, 69
Descurainia, 29

pinnata pinnata, 29

sophia, 29

Desmanthus illinoensis, 47, 105

Desmodium, 47, 105

canadense, 47

canescens, 47

ciliare, 47

cuspidatum var. cuspidatum, 47

fernaldii, 47

glabellum, 47

"glutinosum, 47

humifusum, 47

laevigatum, 47

lineatum, 47

marilandicum, 47

"nudiflorum, 47

nuttallii, 47

obtusum, 47

ochroleucum, 47

paniculatum var.

paniculatum, 47

"pauciflorum, 47

perplexum, 47

"rigidum, 47

rotundifolium, 47

sessilifolium, 47

strictum, 47

tenuifolium, 47, 105

viridiflorum, 47

Deutzia, 52

gracilis, 52

scabra, 52

"Dianthera americana, 9

Dianthus, 33, 102

armeria, 33

barbatus, 33

deltoides, 33, 102

plumarius, 33

"prolifer, 33

"Diarina festucoides, 71, 110

Diarrhena, 71, 110

americana, 71, 110

obovata, 71,110

Dicentra, 66

canadensis, 66

cucullaria, 66

eximia, 66

Dichanthelium, 71-73, 110

aciculare, 71

aculeatum, 71

acuminatum, 71

"acuminatum ssp. columbianum, 71

*acuminatum ssp. implicatum, 72

*acuminatum ssp. leucothrix, 72

*acuminatum ssp. lindheimeri, 72

"acuminatum ssp. longiligulatum, 72 


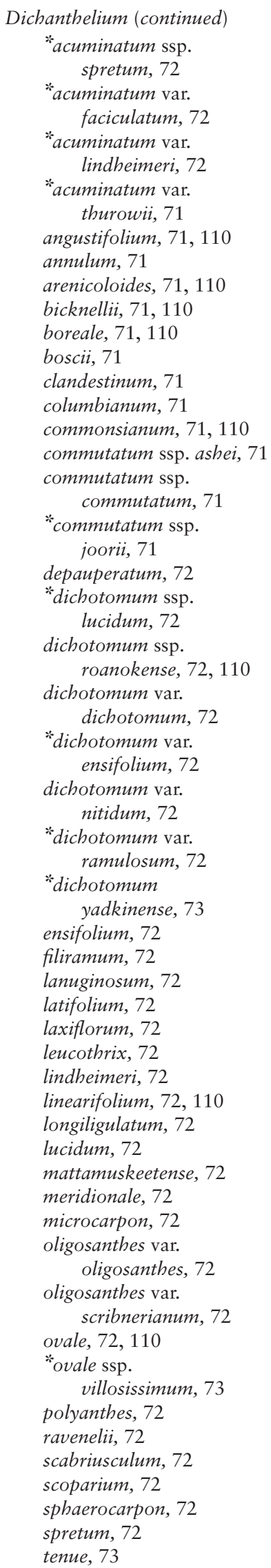

villosissimum, 73

wrightianum, 73

yadkinense, 73

Dichondra, 36, 103

carolinensis, 36, 103

"evolvulacea, 36, 103

micrantha, 36

"repens, 36

Diervilla, 32

"diervilla, 32

lonicera, 32

Digitalis, 67, 109

lanata, 67, 109

lutea, 67, 109

purpurea, 67

Digitaria, 73

*adscendens, 73

ciliaris, 73

cognata, 73

filiformis, 73

ischaemum, 73

"ischaemum var. mississippiensis, 73

longiflora, 73

sanguinalis, 73

"sanguinalis var. ciliaris, 73

villosa, 73

Dinebra retroflexa, 73

"Diodella, 88

teres, 88

teres var. hirsutior, 88

Diodia, 88

"hirsuta, 88

teres, 88

*tetragona, 88

virginiana, 88

*virginiana var. latifolia, 88

Dioscorea, 43

"batatas, 43

"glauca, 43

"birticaulis, 43

*oppositifolia, 43

polystachya, 43

"quaternata, 43

villosa, 43

"villosa var. glabrifolia, 43

"villosa var. hirticaulis, 43

Diospyros virginiana, 43

Diphasiastrum, 5

digitatum, 5

tristachyum, 5

Diplachne, 73

dubia, 73

fusca, 73

"maritima, 73

Diplazium, 6

"acrostichoides, 6

"pycnocarpon, 6

Diplotaxis, 29-30

siifolia, 29

tenuifolia, 30

Dipsacus, 32

fullonum, 32

laciniatus, 32 sativus, 32

*sylvestris, 32

Dirca palustris, 93

"Disporum, 58, 107

lanuginosum, 58

maculatum, 58, 107

Distichlis spicata, 73

"Dodecatheon meadia, 83

Doellingeria, 19, 99

bumilis, 19

infirma, 19, 99

sericocarpoides, 19, 99

umbellata, 19

"Dolichos lablab, 48, 105

"Dondia linearis, 12

*Donia squarrosa, 20

Draba, 30

ramosissima, 30

verna, 30

Dracocephalum, 55, 56, 106

*denticulatum, 56

parviflorum, 55, 106

*virginianum, 56

Drosera, 43

brevifolia, 43

capillaris, 43

filiformis, 43

intermedia, 43

rotundifolia, 43

"Drymocallis arguta, 87

Dryopteris, 6, 8

*atropalustris, 6

campyloptera, 6

carthusiana, 6

celsa, 6

clintoniana, 6

cristata, 6

goldiana, 6

intermedia, 6

marginalis, 6

"noveboracensis, 8

"spinulosa, 6

"thelypteris, 8

*Duchesnea indica, 87

Dulichium arundinaceum, 41

Dysphania, 11

ambrosioides, 11

botrys, 11

plantaginella, 11

pumilio, 11

Dyssodia papposa, 19

Echinacea, 19

pallida, 19

purpurea, 19

Echinochloa, 73

colona, 73

crus, 73

*crus-galli var. frumentacea, 73

*crus-galli var. mitis, 73

"crus-galli var. zelayensis, 73

crus-pavonis, 73 frumentacea, 73

*microstachya, 73

muricata var. microstachya, 73

muricata var. muricata, 73 walteri, 73

Echinocystis lobata, 36

Echinodorus, 9, 10, 98

cordifolius ssp. cordifolius, 9

"radicans, 9

*tenellus, 10, 98

Echinops sphaerocephalus, 19, 99

Echium, 27

italicum, 27

vulgare, 27

Eclipta, 19

*alba, 19

*erecta, 19

prostrata, 19

*Edrastima uniflora, 89

Egeria densa, 52

"Eichhornia crassipes, 82

Elaeagnus, 44, 104

angustifolia, 44

commutata, 44

multiflora, 44, 104

pungens, 44

umbellata, 44

Elatine, 44

americana, 44

minima, 44

triandra, 44

"triandra var. americana, 44

"Elatinoides elatine, 67

Eleocharis, 41-42, 104

acicularis, 41

albida, 41

ambigens, 41

*calva, 41

"capitata var. dispar, 104

"capitata var. pseudoptera, 41

*capitata var.

verrucosa, 42

compressa var. compressa, 41

engelmannii, 41

equisetoides, 41

erythropoda, 41

fallax, 41, 104

"flaccida, 41

flavescens, 41, 104

"flavescens var.

olivacea, 41

geniculata, 41, 104

halophila, 41

intermedia, 41

melanocarpa, 41

microcarpa var. filiculmis, 41

obtusa, 41, 104

*ochreata, 41

olivacea, 41, 104 
ovata, 41, 104

palustris, 41

*palustris var. major, 41

parvula, 41

quadrangulata, 41

robbinsii, 41

rostellata, 41

"simplex, 42

"smallii, 41

tenuis var. pseudoptera, 41

tenuis var. tenuis, 42

tenuis var. verrucosa, 42

*torreyana, 41

tortilis, 42

tricostata, 42

tuberculosa, 42

Elephantopus, 19

carolinianus, 19

nudatus, 19

tomentosus, 19

Eleusine indica, 73

Eleutherococcus sieboldianus, 15

Ellisia nyctelea, 27

Elodea, 52

canadensis, 52

*densa, 52

nuttallii, 52

Elymus, 73-74, 110-111

canadensis, 73

glabriflorus var. australis, 73

glabriflorus var. glabriflorus, 73

"halophilus, 74

hystrix, 73

*hystrix var.

bigelovianus, 73

macgregorii, 73

repens, 73

riparius, 73

"striatus, 74

*striatus var. villosus, 73

trachycaulus ssp.

glaucus, 73, 110

trachycaulus ssp. subsecundus, 73, 111

villosus, 73

*villosus var. arkansanus, 73

virginicus var. halophilus, 74

"virginicus var. hirsutiglumis, 74

virginicus var.

intermedius, 74

virginicus var. virginicus, 74

"Elytrigia repens, 73

Endodeca serpentaria, 16

Enneapogon desvauxii, 74

Epifagus virginiana, 65

Epigaea repens, 44

Epilobium, 62, 108

*angustifolium, 62

ciliatum, 62 coloratum, 62

"glandulosum, 62

hirsutum, 62

leptophyllum, 62, 108

parviflorum, 62

strictum, 62, 108

Epipactis helleborine, 63

Equisetum, 6-7

arvense, 6

fluviatile, 6

byemale ssp. affine, 7

"hyemale var. affine, 7

"praealtum, 7

"robustum, 7

sylvaticum, 7

"sylvaticum var. multiramosum, 7

Eragrostis, 74

*atherstonii, 74

barrelieri, 74

"campestris, 74

capillaris, 74

cilianensis, 74

curvula, 74

cylindriflora, 74

*diffusa, 74

*eragrostis, 74

frankii, 74

hirsuta, 74

*hirsuta var. laevivaginata, 74

*horizontalis, 74

hypnoides, 74

*major, 74

mexicana ssp. virescens, 74

minor, 74

*neomexicana, 74

pectinacea var. pectinacean, 74

pilosa, 74

"poaeoides, 74

"purshii, 74

refracta, 74

spectabilis, 74

"suaveolens, 74

trichodes, 74

trichophora, 74

unioloides, 74

Eranthis hyemalis, 84

Erechtites, 19

hieraciifolius, 19

"hieraciifolius var. intermedia, 19

"hieraciifolius var. praealta, 19

Erianthus, 77, 78, 79, 111

*alopecuroides, 77

"brevibarbis var. brevibarbis, 78, 111

"brevibarbis var. contortus, 78, 111

*coarctatus, 78

"contortus, 78

"contortus var. compactus, 78 *divaricatus, 77

*giganteus, 78

"ravennae, 79

"saccharoides, 78

"smallii, 78

*strictus, 77

Erigenia bulbosa, 13

Erigeron, 18, 19

annuus, 19

"canadensis, 18

philadelphicus, 19

pulchellus var. branniae, 19

pulchellus var. pulchellus, 19

"ramosus, 19

*strigosus var.

beyrichii, 19

strigosus var. strigosus, 19

Eriocaulon, 45

aquaticum, 45

compressum, 45

decangulare var. decangulare, 45

parkeri, 45

"septangulare, 45

Eriochloa, 74

acuminata, 74

"acuminata var. minor, 74

*gracilis, 74

punctata, 74

Eriophorum, 42

gracile, 42

virginicum, 42

Erodium cicutarium, 51

"Erophila verna, 30

Eruca, 30

"sativa, 30

vesicaria, 30

Erucastrum gallicum, 30

Eryngium, 13

aquaticum, 13

campestre, 13

yuccifolium var. yuccifolium, 13

Erysimum, 30, 101

asperum, 30, 101

capitatum, 30, 101

cheiranthoides, 30

inconspicuum, 30, 101

*officinale, 30

repandum, 30

Erythronium, 57-58, 107

albidum, 57

americanum, 58, 107

*americanum ssp. harperi, 58

umbilicatum, 58, 107

Eubotrys racemosa, 44

"Eulalia, 75

viminea, 75

viminea var. variabilis, 75

Euonymus, 34

alatus, 34

americanus, 34

atropurpurea, 34

europaeus, 34 fortunei var. radicans, 34

"radicans, 34

"Eupatoriadelphus, 20

dubius, 20

fistulosus, 20

maculatus, 20

purpureus, 20

Eupatorium, 17, 18, 19-20, 100

"ageratoides, 17

album, 19

*album var. vaseyi, 20

altissimum, 19

*americanum, 20

*amoenum, 20

"aromaticum, 17

cannabinum, 19

capillifolium, 19

"coelestinum, 18

*dubium, 20

*fistulosum, 20

godfreyanum, 19

byssopifolium var. byssopifolium, 19

"hyssopifolium var. laciniatum, 20

*latidens, 17

*lecheifolium, 19

leucolepis, 19

"maculatum, 20

perfoliatum, 19

"petaloideum, 19

pilosum, 19

"pubescens, 19

*purpureum, 20

rotundifolium var. ovatum, 19

rotundifolium var. rotundifolium, 19

"rotundifolium var. saundersii, 19

"rugosum, 17

semiserratum, 20, 100

serotinum, 20

sessilifolium var. sessilifolium, 20

"sessilifolium var. vaseyi, 20

"ternifolium, 20

torreyanum, 20

"trifoliatum, 20

vaseyi, 20

*verbenifolium, 19

Euphorbia, 45-46, 105

"arundelana, 46

*chamaesyce, 46

commutata, 45

corollata, 45, 105

*corollata var. zinniiflora, 46, 105

cyathophora, 45

cyparissias, 45

davidii, 45

dentata, 45

"dictyosperma, 46

esula, 45 
Euphorbia (continued) falcata, 45

"glomerifera, 46 belioscopia, 45 herniariifolia, 45 birta, 45

humistrata, 46 hypericifolia, 46 hyssopifolia, 46 *intercedens, 45 ipecacuanhae, 46 lathyris, 46 lucida, 46, 105 maculata, 46 marginata, 46 "marilandica, 45, 46 nutans, 46

*obtusata, 46 peplus, 46

"pilulifera var. discolor, 45 polygonifolia, 46 prostrata, 46 pubentissima, 46, 105 purpurea, 46 spathulata, 46 "supina, 46 vermiculata, 46 "zinniiflora, 46, 105

Eurybia, 20

compacta, 20

divaricata, 20

macrophylla, 20

radula, 20

schreberi, 20

spectabilis, 20

Euthamia, 20

caroliniana, 20

graminifolia, 20

*graminifolia var. nuttallii, 20

gymnospermoides, 20

hirtipes, 20

"microcephala, 20

Eutrochium, 20

dubium, 20

fistulosum, 20

maculatum var. maculatum, 20 purpureum, 20

Evolvulus filipes, 36

Exochorda racemosa, 86

Fagopyrum, 80 esculentum, 80 *sagittatum, 80

Fagus, 50, 105 grandifolia, 50 sylvatica, 50, 105

Falcaria, 13, 98 *sioides, 13, 98 vulgaris, 13, 98

Fallopia, 80, 81, 112 baldschuanica, 80, 112 cilinodis, 80 convolvulus, 80

cristata, 80,112

dumetorum, 80, 112

"japonica, 81

scandens, 80

Fatoua villosa, 61

Festuca, 74, 75

*arundinacea, 75

*brevipila, 74

"elatior, 75

filiformis, 74

"gracilenta, 74

myuros, 74

*nutans, 74

*obtusa, 74

"octoflora var. aristulata, 74

octoflora var. glauca, 74

octoflora var. octoflora, 74

"ovina, 74

*ovina var. capillata, 74

*ovina var. duriuscula, 74

paradoxa, 74

"pratensis, 75

rubra, 74

sciurea, 74

"shortii, 74

subverticillata, 74

"tenella, 74

trachyphylla, 74

Ficaria, 84

verna, 84

*verna ssp. calthifolia, 84

*verna ssp. chrysocephala, 84

*verna ssp. fertilis, 84

*verna ssp. ficariiformis, 84

Ficus carica, 61

Filago vulgaris, 20

Filipendula rubra, 86

Fimbristylis, 42, 104

annua, 42

autumnalis, 42

"baldwiniana, 42

caroliniana, 42

castanea, 42, 104

"geminata, 42

"harperi, 42

perpusilla, 42

puberula var. puberula, 42,104

*spadicea, 42, 104

Firmiana, 59, 108 "platanifolia, 59, 108 simplex, 59, 108

Flaveria trinervia, 20

Floerkea proserpinacoides, 58

Foeniculum vulgare, 13

Forsythia, 62, 108

suspensa, 62

viridissima, 62

Fragaria, 86

"australis, 86

vesca var. vesca, 86

virginiana, 86
Frangula, 85

alnus, 85

caroliniana, 85

Fraxinus, 62

americana, 62

"americana var. curtissii, 62

biltmoreana, 62

caroliniana, 62

*darlingtonii, 62

*lanceolata, 62

"michauxii, 62

nigra, 62

pennsylvanica, 62

"pennsylvanica var. subintegerrima, 62

profunda, 62

"smallii, 62

Froelichia, 11

floridana var. floridana, 11 gracilis, 11

Fuirena, 42

"hispida, 42

pumila, 42

squarrosa, 42

"squarrosa var. hispida, 42

Fumaria officinalis, 66

Gaillardia pulchella var. pulchella, 20

Galactia, 47, 105 brachypoda, 47, 105

"macreei, 47, 105

regularis, 47

volubilis var. volubilis, 47, 105

Galanthus nivalis, 12

Galax urceolata, 43

Galearis spectabilis, 63

Galega officinalis, 47

Galeopsis tetrahit, 55

*Galeorchis spectabilis, 63

Galinsoga, 20

*caracasana, 20

"ciliata, 20

parviflora, 20

quadriradiata, 20

Galium, 88-89, 114

anglicum, 88

aparine, 88

arvense, 88

asprellum, 88

"bermudense, 89

boreale, 88

circaezans, 88

*circaezans var. bypomalacum, 88

*claytonii, 89

concinnum, 88

hispidulum, 89

lanceolatum, 89

latifolium, 89

mollugo, 89

obtusum, 89 "obtusum var. filifolium, 89

odoratum, 89, 114

palustre, 89,114

"parisiense, 88

pedemontanum, 89

pilosum, 89

"pilosum var. puncticulosum, 89

*sherardia, 89

tinctorium var. floridanum, 89

tinctorium var. tinctorium, 89

tricornutum, 89

trifidum, 114

trifidum var. trifidum, 89, 114

triflorum, 89

uniflorum, 89, 114

vernum, 89

Gamochaeta, 20

pensylvanica, 20

purpurea, 20

*Gattenhofia verna, 53

Gaultheria, 44

hispidula, 44

procumbens, 44

Gaura, 62

biennis, 62

"filiformis, 62

longiflora, 62

Gaylussacia, 44

baccata, 44

"bigeloviana, 44

brachycera, 44

dumosa, 44

*dumosa var. bigeloviana, 44

frondosa, 44

"Gemmingia chinensis, 53

Genista tinctoria, 47, 105

Gentiana, 50-51, 105

alba, 50, 105

andrewsii var. andrewsii, 50

autumnalis, 51, 105

catesbyi, 51

clausa, 51

*crinita, 51

*elliottii, 51

linearis, 51

*puberula, 51

puberulenta, 51

saponaria, 51

*ventricosa, 51

villosa, 51

Gentianella, 51

"crinita, 51

quinquefolia ssp. quinquefolia, 51

Gentianopsis crinita, 51

Geranium, 51, 105

carolinianum, 51

columbinum, 51

dissectum, 51 
maculatum, 51, 105

molle, 51

*nepalense var. thunbergii, 51

pratense, 51, 105

pusillum, 51

robertianum, 51

rotundifolium, 51

sibiricum, 51

thunbergii, 51

${ }^{*}$ Gerardia, 65, 109

auriculata, 65

linifolia, 109

maritima, 65

purpurea, 65

setacea, 65

tenuifolia, 65

Geum, 86

aleppicum, 86

"aleppicum var. strictum, 86

canadense, 86

fragarioides, 86

*hirsutum, 86

laciniatum, 86

"laciniatum var. trichocarpum, 86

rivale, 86

*strictum, 86

vernum, 86

virginianum, 86

${ }^{*}$ Gilia, 80

coronopifolia, 80

rubra, 80

Gillenia, 86, 114

stipulata, 86, 114

trifoliata, 86, 114

Glandularia bipinnatifida var. bipinnatifida, 93

Glaucium flavum, 66

"Glaux maritima, 83

Glebionis segetum, 20, 100

Glechoma hederacea, 55

Gleditsia triacanthos, 47

Glossostigma cleistanthum, 66

Glyceria, 74-75, 79, 111

acutiflora, 74

canadensis, 74

*canadensis var. laxa, 74

declinata, 74

fluitans, 74, 111

grandis, 74, 111

laxa, 74

melicaria, 74

obtusa, 75

*pallida, 79

*pallida var. fernaldii, 79

septentrionalis, 75

striata, 75

Glycine, 46, 47

*apios, 46

max, 47

Gnaphalium, 20, 23

"helleri, 23

*obtusifolium, 23 *obtusifolium var. helleri, 23

"purpureum, 20

uliginosum, 20

Gomphrena, 11

*celosioides, 11

globosa, 11

serrata, 11

Gonolobus, 14

"gonocarpos, 14

suberosus var. suberosus, 14

Goodyera, 63

pubescens, 63

repens, 63

tesselata, 63

Gossypium, 59

"herbaceum, 59

hirsutum, 59

*GRAMINEAE, 68-79, 110-111

Gratiola, 67, 68

aurea, 67

neglecta, 67

*pilosa, 68

ramosa, 67

*sphaerocarpa, 67

virginiana, 67

*virginiana var. aestuariorum, 67 viscidula, 67

Greeneochloa coarctata, 74

Grindelia, 20

ciliata, 20

lanceloata, 20

"papposa, 20

squarrosa, 20

*Grossularia, 51

cynosbati, 51

rotundifolia, 51

Guilleminea densa var. aggregata, 11

Guizotia abyssinica, 20

*Gymnadeniopsis, 64, 108 clavellata, 64 integra, 64, 108

Gymnocarpium, 6, 97 appalachianum, 6, 97 dryopteris, 6, 97

Gymnocladus dioica, 47

Gymnopogon, 75 ambiguus, 75 brevifolius, 75

Gypsophila, 33 paniculata, 33 pilosa, 33

*Habenaria, 63, 64, 108 blephariglottis, 64

ciliaris, 64

clavellata, 64

cristata, 64

fimbriata, 64

flava var. herbiola, 64 integra, 64, 108

lacera, 64

peramoena, 64

viridis var. bracteata, 63

Hackelia virginiana, 27

Hackelochloa granularis, 75

Halesia, 92

carolina, 92

tetraptera, 92

Hamamelis virginiana, 52

"Haplopappus ciliatus, 20

*Harperella, 13

fluviatilis, 13 nodosa, 13

"Hasteola suaveolens, 23

Hedeoma, 55, 106 hispida, 55, 106 pulegioides, 55

Hedera, 15

helix, 15

hibernica, 15

*Hedyotis, 89

caerulea, 89

crassifolia, 89

longifolia, 89

longifolia var. tenuifolia, 89

minima, 89

minor, 89

nuttalliana, 89

patens, 89

purpurea, 89

uniflora, 89

Helanthium tenellum, 10, 98

Helenium, 20-21

amarum var. amarum, 20

autumnale var. autumnale, 21

"autumnale var. pubescens, 21

flexuosum, 21

"latifolium, 21

"nudiflorum, 21

*tenuifolium, 20

*Helianthemum, 34

bicknellii, 34

canadense, 34

propinquum, 34

Helianthus, 21, 100

"alienus, 21

angustifolius, 21

"angustifolius var. nemorosus, 21

annuus, 21

"cucumerifolius, 21

debilis ssp. cucumerifolius, 21

decapetalus, 21

divaricatus, 21, 100

*dowellianus, 21

giganteus, 21

grosseserratus, 21

hirsutus, 21

"hirsutus var. stenophyllus, 21

*hirsutus var. trachyphyllus, 21

laevigatus, 21 maximiliani, 21

microcephalus, 21, 100

mollis, 21

"montanus, 21

occidentalis ssp. occidentalis, 21

pauciflorus, 21

salicifolius, 21, 100

"stenophyllus, 21

strumosus, 21

"tracheliifolius, 21

tuberosus, 21

Heliopsis helianthoides var. helianthoides, 21

Heliotropium, 28

curassavicum, 28

europaeum, 28

indicum, 28

procumbens, 28

Helleborus, 84

xhybridus, 84

viridis, 84

Helminthotheca echioides, 21, 100

Helonias bullata, 60

Hemerocallis, 16

*flava, 16

fulva, 16

lilioasphodelus, 16

*Hemianthus

micranthemoides, 58

*Hemicarpha micrantha, 41

*Hepatica, 83

acutiloba, 83

americana, 83

Heracleum, 13

"lanatum, 13

mantegazzianum, 13

maximum, 13

Hermbstaedtia, 11

*elegans, 11

odorata, 11

Herniaria, 33

*cinerea, 33

glabra, 33

birsuta var. cinerea, 33

*incana, 33

*Herpestis rotundifolia, 67

Hesperis matronalis, 30

Heteranthera, 82

dubia, 82

multiflora, 82

pauciflora, 82

reniformis, 82

Heterosperma pinnatum, 21

Heterotheca, 18, 21, 23

*latifolia, 21

*mariana, 18

nervosa var. nervosa, 23

subaxillaris, 21

*subaxillaris var. latifolia, 21

Heuchera, 91, 115

alba, 91

americana, 91 
Heuchera (continued)

*americana var. hispida, 91

*alycosa, 91

"curtisii, 91

hispida, 91

"lancipetala, 91

"macrorhiza, 91, 115

pubescens, 91

villosa var. villosa, 91, 115

Hexalectris spicata, 63

"Hexasepalum teres, 88

"Hexastylis virginica, 16

Hibiscus, 59

*incanus, 59

laevis, 59

*militaris, 59

moscheutos, 59

*moscheutos var. incanus, 59

*moscheutos var. palustris, 59

*oculiroseus, 59

syriacus, 59

trionum, 59

Hieracium, 21

aurantiacum, 21

caespitosum, 21

gronovii, 21

marianum, 21

paniculatum, 21

pilosella, 21

piloselloides, 21

"pretense, 21

scabrum, 21

traillii, 21

venosum, 21

"Hierochloe, 69

hirta, 69

odorata, 69

Holcus, 75, 78

"halepensis, 78

lanatus, 75

*sorghum, 78

Holosteum umbellatum, 33

*Homalocenchrus, 75

hexandrus, 75

lenticularis, 75

oryzoides, 75

virginicus, 75

Homalosorus pycnocarpos, 6

Honckenya peploides robusta, 33

Hordeum, 75, 111

brachyantherum ssp.

brachyantherum,

75,111

"hystrix, 75

jubatum, 75

"leporinum, 75

marinum, 75

murinum, 75

*murinum ssp. leporinum, 75

"nodosum, 75, 111 pratense, 111

pusillum, 75

vulgare, 75

Hosta, 16, 99

lancifolia, 16, 99

ventricosa, 16

Hottonia inflata, 82

"Houpoea tripetala, 59

Houstonia, 89, 114

caerulea, 89

lanceolata, 89, 114

longifolia var. compacta, 89

longifolia var. longifolia, 89, 114

"longifolia var. tenuifolia, 89

micrantha, 89

"purpurea var. calycosa, 89, 114

purpurea var. purpurea, 89

pusilla, 89

serpyllifolia, 89

tenuifolia, 89

Houttuynia cordata, 90, 115

Hudsonia, 34

ericoides, 34

tomentosa, 34

Humulus, 31

japonicus, 31

lupuloides, 31

lupulus, 31

Huperzia, 5

lucidula, 5

porophila, 5

"Hybanthus concolor, 94

"Hydatica petiolaris, 91, 115

Hydrangea, 52

arborescens, 52

*arborescens var. cordata, 52

Hydrastis canadensis, 84

Hydrilla verticillata, 52

Hydrocotyle, 15-16

americana, 15

"australis, 16

"canbyi, 16

"prolifera, 16

ranunculoides, 15

sibthorpioides, 15

umbellata, 16

verticillata, 16

*verticillata var. triradiata, 16

Hydrolea quadrivalvis, 52

Hydrophyllum, 28

canadense, 28

macrophyllum, 28

virginianum, 28

"Hydrotrida caroliniana, 66, 109

Hylodesmum, 47

glutinosum, 47

nudiflorum, 47

pauciflorum, 47
Hylotelephium, 36 telephioides, 36 telephium, 36

Hymenachne hemitomon, 75

Hyoscyamus niger, 91

Hypecoum pendulum, 66

Hypericum, 52-53, 106

"acutifolium, 53

adpressum, 52

androsaemum, 52

ascyron ssp.

pyramidatum, 52, 106

boreale, 52

canadense, 52

crux-andreae, 52

densiflorum, 52

*denticulatum var. acutifolium, 53

denticulatum var. denticulatum, 52

drummondii, 52

ellipticum, 52

fraseri, 52

gentianoides, 53

*glomeratum, 52

gymnanthum, 53

hypericoides, 53

mitchellianum, 53, 106

mutilum, 53

perforatum, 53

prolificum, 53

punctatum, 53

*stans, 52

stragulum, 53

*stragulum, 53

"subpetiolatum, 53

tubulosum, 53, 106

virgatum, 53

*virgatum var. acutifolium, 53

virginicum, 53

walteri, 53, 106

Hypochaeris radicata, 21

"Hypopitys, 44

americana, 44

hypopitys, 44

lanuginosa, 44

Hypoxis hirsuta, 53

"Hystrix patula, 73

Ilex, 14-15

"ambigua var. montana, 15

*ambigua var. monticola, 15

crenata, 14

decidua, 14

glabra, 15

laevigata, 15

montana, 15

"monticola, 15

"monticola var. mollis, 15

mucronata, 15 opaca, 15

verticillata, 15

Impatiens, 26

"aurea, 26

balsamina, 26

"biflora, 26

capensis, 26

pallida, 26

Imperatoria ostruthium, 13

Indigofera trifoliata, 47

Inula helenium, 21

Ionactis linariifolia, 21

Ipomoea, 36, 103

"barbigera, 36

coccinea, 36

hederacea, 36

*hederacea var. integriuscula, 36

lacunosa, 36

nil, 36

pandurata, 36

purpurea, 36

quamoclit, 36, 103

*vulgaris, 36, 103

Ipomopsis rubra, 80

Iresine rhizomatosa, 11

Iris, 53, 106

cristata, 53

domestica, 53

ensata, 53, 106

germanica, 53

*kaempferi, 53, 106

prismatica, 53

pseudacorus, 53

*shrevei, 53

verna, 53

*verna var. smalliana, 53

versicolor, 53

virginica, 53

*virginica var. shrevei, 53

"Isanthus brachiatus, 57

Ischaemum, 75

*iliare, 75

indicum, 75

rugosum, 75

"Isnardia palustris, 62

Isoetes, 3, 5, 97

engelmannii, 5

riparia, 5

*saccharata, 3, 5

tuckermanii, 5, 97

Isotrema macrophyllum, 16

Isotria, 64

medeoloides, 64

verticillata, 64

Itea virginica, 53

Iva, 21

annua, 21

frutescens $\mathrm{ssp}$. frutescens, 21

*frutescens ssp. oraria, 21

Jaltomata procumbens, 91

Jasione montana, 31, 101 
Jasminum nudiflorum, 62, 108 Jeffersonia diphylla, 27

Juglans, 54

ailantifolia, 54

cinerea, 54

nigra, 54

Juncus, 54, 106

*abortivus, 54

acuminatus, 54

"acuminatus var. debilis, 54 anthelatus, 54

*arcticus ssp. balticus, 54

*arcticus var. littoralis, 54 articulatus, 54

balticus var. littoralis, 54

biflorus, 54

brachycarpus, 54

brachycephalus, 54, 106

brevicaudatus, 54

bufonius, 54

caesariensis, 54

canadensis, 54

compressus, 54

coriaceus, 54

"crassifolius, 54

debilis, 54

dichotomus, 54

diffusissimus, 54

dudleyi, 54

effusus ssp. solutus, 54

elliottii, 54

filiformis, 54, 106

gerardii, 54

longii, 54

marginatus, 54

"marginatus var.

biflorus, 54

megacephalus, 54

militaris, 54

pelocarpus, 54

"platyphyllus, 54

polycephalos, 54, 106

"polycephalus, 54, 106

pylaei, 54

repens, 54

roemerianus, 54

scirpoides var. compositus, 54

scirpoides var. scirpoides, 54

secundus, 54

"setaceus, 54

subcaudatus, 54, 106

tenuis, 54

"tenuis var. anthelatus, 54

torreyi, 54

"trifidus, 54

validus, 54, 106

Juniperus, 8

communis var. depressa, 8 virginiana var. virginiana, 8

"Jussiaea, 62

decurrens, 62

diffusa, 62 leptocarpa, 62

pilosa, 62

repens, 62

Justicia americana, 9

Kallstroemia parviflora, 95

Kalmia, 44 angustifolia, 44 latifolia, 44

Kalopanax septemlobus, 16

Kellochloa verrucosa, 75

Kickxia elatine, 67

Knautia arvensis, 32, 102

"Kochia scoparia, 11

Koeleria, 75, 77 macrantha, 75

"nitida, 75

"pleoides, 77

Koelreuteria paniculata, 90

"Koniga maritima, 30

Kosteletzkya, 59

*althaeifolia, 59

pentacarpos, 59

*virginica, 59

Krigia, 21, 100

biflora var. biflora, 21, 100

cespitosa, 21

dandelion, 21

virginica, 21

${ }^{*}$ Kubnia, 18

eupatorioides, 18

glutinosa, 18

kubnia, 18

mosieri, 18

Kummerowia, 48

stipulacea, 48 striata, 48

"Kyllinga, 40, 41

gracillima, 40

pumila, 41

"LABIATAE, 54-57, 106

Lablab purpureus, 48, 105

"Labsana capillaris, 19

Lachnanthes caroliniana, 51

"Lacinaria, 22

spicata, 22

squarrosa, 22

Lactuca, 21-22

biennis, 21

canadensis, 21

floridana, 22

"floridana var. villosa, 22

hirsuta, 22

*sagittifolia, 21

saligna, 22

"scariola, 22

serriola, 22

*villosa, 22

Lagerstroemia indica, 58

Lamiastrum galeobdolon, 55
Lamium, 55

album, 55

amplexicaule, 55

*galeobdolon, 55

maculatum, 55

purpureum, 55

Landoltia punctata, 15

Laportea canadensis, 93

Lappula, 27, 28

"echinata, 28

squarrosa, 28

*virginiana, 27

Lapsana communis, 22

Larix, 8

decidua, 8

laricina, 8

"Lasiococcus dumosus, 44

Lathyrus, 48

aphaca, 48

birsutus, 48

inconspicuus, 48

latifolius, 48

palustris, 48

sativus, 48

venosus, 48

Lavatera trimestris, 59, 108

Lechea, 34

"leggettii, 34

maritima var. virginica, 34

minor, 34

mucronata, 34

pulchella, 34

"pulchella var. moniliformis, 34

racemulosa, 34

tenuifolia, 34

"villosa, 34

Leersia, 75

bexandra, 75

lenticularis, 75

oryzoides, 75

virginica, 75

"Legousia perfoliata, 31

*LEGUMINOSAE, 46, 105

Lemna, 15

aequinoctialis, 15

gibba, 15

minor, 15

obscura, 15

perpusilla, 15

trisulca, 15

turionifera, 15

valdiviana, 15

Lens culinaris, 48

Leontodon, 22, 26

autumnalis, 22

"erythrospermum, 26

hispidus, 22

*leysseri, 22

saxatilis ssp. saxatilis, 22

*taraxacum, 26

Leonurus, 55

cardiaca, 55

*marrubiastrum, 55
Lepidium, 30

campestre, 30

densiflorum var. densiflorum, 30

didymum, 30

draba, 30

graminifolium, 30

hyssopifolium, 30

oblongum, 30

perfoliatum, 30

ruderale, 30

virginicum, 30

"Leptamnium virginianum, 65

"Leptandra virginica, 68

"Leptilon canadense, 18

"Leptochloa, 73

dubia, 73

fascicularis, 73

maritima, 73

"Leptodactyon pungens, 80

"Leptoloma cognata, 73

Lespedeza, 48

angustifolia, 48

bicolor, 48

capitata, 48

cuneata, 48

frutescens, 48

hirta ssp. hirta, 48

*intermedia, 48

procumbens, 48

repens, 48

*stipulacea, 48

*striata, 48

stuevei, 48

thunbergii, 48

violacea, 48

*violacea, 48

virginica, 48

Leucanthemum, 22

"leucanthemum, 22

vulgare, 22

Leucojum aestivum, 12

Leucospora multifida, 67

Leucothoe, 44

axillaris, 44

"axillaris var. editorum, 44

*atesbaei, 44

fontanesiana, 44

"racemosa, 44

*walteri, 44

Liatris, 22

"graminifolia, 22

helleri, 22

pilosa, 22

scariosa, 22

spicata var. spicata, 22

squarrosa var. squarrosa, 22

"turgida, 22

Ligularia dentata, 22

Ligusticum canadense, 13

Ligustrum, 62

"amurense, 62

japonicum, 62 
Ligustrum (continued)

lucidum, 62

obtusifolium var. obtusifolium, 62

*obtusifolium var. regelianum, 62

*obtusifolium var. suave, 62 ovalifolium, 62

quiboui, 62

sinense, 62

vulgare, 62

Lilaeopsis chinensis, 13

Lilium, 58, 107

canadense, 58

"canadense var. editorum, 58

canadensis, 107

grayi, 58, 107

lancifolium, 58

philadelphicum, 58

superbum, 58

*tigrinum, 58

"Limniris, 53, 106

ensata, 53, 106

prismatica, 53

pseudacorus, 53

versicolor, 53

virginica, 53

Limnobium spongia, 52

"Limodorum tuberosum, 63

Limonium, 68

carolinianum, 68

*nashii, 68

Limosella, 67

australis, 67

*subulata, 67

Linanthus pungens, 80

Linaria, 67

canadensis, 67

vulgaris, 67

Lindera, 57

benzoin, 57

*benzoin var. pubescens, 57

Lindernia, 58, 108

*anagallidea, 58

dubia, 58, 108

*dubia var. anagallidea, 58

Linnaea borealis, 32, 102, 111

Linum, 58, 107

floridanum var.

floridanum, 58, 107

intercursum, 58, 107

medium var. texanum, 58

striatum, 58

sulcatum, 58

usitatissimum, 58

virginianum, 58

*virginianum var. medium, 58

Liparis, 64

liliifolia, 64

loeselii, 64

"Lipocarpha micrantha, 41
*Lippia, 93

lanceolata, 93

nodiflora, 93

Liquidambar styraciflua, 10

Liriodendron tulipifera, 58

Liriope, 16, 99 graminifolia, 16, 99

muscari, 16, 99

spicata, 16, 99

"Listera, 64

australis, 64

cordata, 64

smallii, 64

Lithospermum, 27, 28, 101

*arvense, 27

canescens, 28

latifolium, 28

molle, 28, 101

parviflorum, 28, 101

virginianum, 28

Litsea aestivalis, 57

Lobelia, 31, 102

*bracteata, 31

canbyi, 31

cardinalis, 31

dortmanna, 31, 102

elongata, 31

glandulosa, 31, 102

inflata, 31

*leptostachys, 31

nuttallii, 31

puberula, 31

siphilitica, 31

spicata, 31

*spicata var. campanulata, 31

*spicata var. leptostachys, 31

"spicata var. scaposa, 31

Lobularia maritima, 30

Lolium, 75

arundinaceum, 75

"multiflora, 75

perenne, 75

"perenne ssp. multiflorum, 75

pratense, 75

temulentum, 75

Lonicera, 32

canadensis, 32

dioica, 32

*dioica var. glaucescens, 32

fragrantissima, 32

japonica, 32

maackii, 32

morrowii, 32

sempervirens var. sempervirens, 32

standishii, 32

tatarica, 32

xylosteum, 32

"Lophiris cristata, 53

"Lophotocarpus spongiosus, 10
Lorinseria areolata, 6

Lotus, 48

corniculatus, 48

"glaber, 48

tenuis, 48

Ludwigia, 62-63, 108

alternifolia, 62

"alternifolia var. linearifolia, 62

brevipes, 62, 108

decurrens, 62

glandulosa, 62

hexapetala, 62

hirtella, 62

leptocarpa, 62

linearis, 62

palustris, 62

"peploides ssp. glabrescens, 62

peploides var. glabrescens, 62

sphaerocarpa, 62

virgata, 63

Lunaria, 30

annua, 30

rediviva, 30

Lupinus, 48

"gracilis, 48

"nuttallii, 48

*perennis ssp. gracilis, 48

perennis ssp. perennis, 48

polyphyllus, 48

Luzula, 54

acuminata, 54

*acuminata var. carolinae, 54

bulbosa, 54

echinata, 54

multiflora ssp. multiflora, 54

*Lychnis, 33, 34, 102

alba, 34

coronaria, 33

dioica, 33

drummondii, 33, 102

flos-cuculi, 34

saponaria, 33

Lycium, 92

barbarum, 92

chinense, 92

"halimifolium, 92

"Lycopersicon esculentum, 92

Lycopodiella, 5

alopecuroides, 5

appressa, 5

inundata, 5

"Lycopodioides apoda, 5

Lycopodium, 5

"adpressum, 5

*alopecuroides, 5

"annotinum, 5

*carolinianum, 5

clavatum, 5

"complanatum var. flabelliforme, 5 *dendroideum, 5

*digitatum, 5

*flabelliforme, 5

"bickeyi, 5

*lucidulum, 5

*obscurum, 5

*obscurum var. dendroideum, 5

"porophilum, 5

*tristachyum, 5

"Lycopsis arvensis, 27

Lycopus, 55

americanus, 55

amplectens, 55

europaeus, 55

"pubens, 55

rubellus, 55

*sessilifolius, 55

uniflorus, 55

*velutinus, 55

virginicus, 55

Lygodium palmatum, 7

Lyonia, 44

ligustrina, 44

"ligustrina var. foliosiflora, 44

mariana, 44

Lysimachia, 82-83

arvensis, 82

borealis, 82

ciliata, 82

foemina, 82

hybrida, 83

japonica, 83

lanceolata, 83

maritima, 83

minima, 83

nummularia, 83

punctata, 83

quadriflora, 83

quadrifolia, 83

terrestris, 83

thyrsiflora, 83

vulgaris, 83

Lythrum, 58

alatum, 58

byssopifolia, 58

lineare, 58

salicaria, 58

Macleaya cordata, 66

Maclura pomifera, 61

*Macuillamia rotundifolia, 67

Madia sativa, 22

Magnolia, 58-59

acuminata, 58

*acuminata var. cordata, 59

*foetida, 59

grandiflora, 59

macrophylla, 59

tripetala, 59

virginiana, 59

Mahonia bealei, 27 
Maianthemum, 16 canadense, 16 racemosum, 16 stellatum, 16

Malaxis unifolia, 64

Malus, 86-87, 114 angustifolia, 86 baccata, 86, 114 "bracteata, 86 coronaria, 86 "domestica, 87 "glabrata, 86 *glaucescens, 86 ioensis var. ioensis, 86,114

*lancifolia, 86 prunifolia, 87 pumila, 87 *sieboldii, 87 "sylvestris, 87 toringo, 87

Malva, 59, 108 moschata, 59 neglecta, 59 parviflora, 59 pusilla, 59, 108 *rotundifolia, 59 sylvestris, 59 verticillata, 59, 108

*Manfreda, 16, 99 tigrina, 16, 99 virginica, 16, 99

"Manisuris rugosa, 75

"Mappia origanoides, 55

"Marginaria polypodioides, 7

*Mariana mariana, 24

"Marilaunidium hispidum, 28

"Mariscus mariscoides, 40

Marrubium vulgare, 55

Marshallia, 22, 100 grandiflora, 22, 100 pulchra, 100

Marsilea quadrifolia, 7

*Martusia mariana, 47

"Martynia louisianica, 59

*Maruta cotula, 17

Matelea, 14, 99 carolinensis, 14 decipiens, 14, 99 "gonocarpos, 14 obliqua, 14

Matricaria, 22, 26, 101 chamomilla, 22 discoidea, 22 *inodora, 26, 101 *maritima, 26

"matricarioides, 22 "recutita, 22

Matteuccia, 7 struthiopteris, 7 "struthiopteris var. pensylvanica, 7

Maurandya antirrbiniflora, 67
Mazus, 59

*japonicus, 59

miquelli, 59

pumilus, 59

Mecardonia acuminata var. acuminata, 67

Medeola virginiana, 58

Medicago, 48 heldreichii, 48

lupulina, 48

minima, 48

monspeliaca, 48

orbicularis, 48

sativa, 48

scutellata, 48

"Megalodonta beckii, 17, 99

Melampodium, 22

"hispidum, 22

sericeum, 22

Melampyrum, 65

lineare, 65

"lineare var. americanum, 65

*lineare var. latifolium, 65

*lineare var. pectinatum, 65

"Melanthium, 60

bybridum, 60

latifolium, 60

parviflorum, 60

virginicum, 60

Melia azedarach, 60

Melica, 75

mutica, 75

nitens, 75

Melilotus, 48

albus, 48

officinalis, 48

Melinis repens, 75

Melissa officinalis, 55

Melochia corchorifolia, 59

Melothria, 36

"microcarpa, 36

"nashii, 36

pendula, 36

*pendula var. aspera, 36

Menispermum canadense, 60

Mentha, 55

aquatica, 55

arvensis, 55

canadensis, 55

"gentilis, 55

longifolia, 55

pulegium, 55

spicata, 55

suaveolens, 55

*sylvestris, 55

Menyanthes trifoliata, 60

Menziesia pilosa, 44

Mercurialis annua, 46

Mertensia virginica, 28

${ }^{*}$ Mesadenia atriplicifolia, 17

*Metamagnolia macrophylla, 59

Micranthemum micranthemoides, 58
Micranthes, 91, 115

micranthidifolia, 91

pensylvanica, 91

petiolaris, 91, 115

virginiensis, 91

Microstegium vimineum, 75

Microthlaspi perfoliatum, 30

Mikania scandens, 22

Milium effusum, 75

Mimosa pudica, 48

Mimulus, 66

alatus, 66

ringens var. ringens, 66

Minuartia, 33

caroliniana, 33

glabra, 33

michauxii var. michauxii, 33

Mirabilis, 61

jalapa, 61

nyctaginea, 61

Miscanthus sinensis, 75

Mitchella repens, 89

Mitella diphylla, 91

Mnesithea rugosa, 75

Modiola caroliniana, 59

Moehringia lateriflora, 33

Moenchia erecta erecta, 33

Mollugo, 61

gracillima, 61

nudicaulis, 61

verticillata, 61

Monarda, 55-56

clinopodia, 55

didyma, 55

fistulosa var. fistulosa, 55

fistulosa var. mollis, 56

media, 56

*mollis, 56

punctata var. punctata, 56

*scabra, 56

*Monniera, 67

acuminata, 67

monnieri, 67

*Mononeuria glabra, 33

Monotropa, 44

"brittonii, 44

bypopitys, 44

uniflora, 44

Monotropsis odorata, 44

Montia linearis, 61

Moorochloa eruciformis, 75

Morella, 61

caroliniensis, 61

cerifera, 61

pensylvanica, 61

Morus, 61

alba, 61

rubra, 61

Mosla scabra, 56

Mublenbergia, 75-76, 111

asperifolia, 75

"brachyphylla, 75, 111

bushii, 75, 111

capillaris, 76 "diffusa, 76

frondosa, 76

glabriflora, 76

glomerata, 76, 111

mexicana, 76

racemosa, 76, 111

schreberi, 76

*schreberi var. palustris, 76

sobolifera, 76

sylvatica, 76

tenuiflora, 76

torreyana, 76

*umbrosa, 76

uniflora, 76

*Mulgedium, 22

floridanum, 22

villosum, 22

Murdannia keisak, 35

*Muricauda dracontium, 15

"Muscadinia rotundifolia, 95

Muscari, 16

botryoides, 16

neglectum, 16

*racemosum, 16

Myosotis, 28

arvensis, 28

discolor, 28

laxa, 28

macrosperma, 28

scorpioides, 28

stricta, 28

verna, 28

*verna var. macrosperma, 28

"versicolor, 28

*virginica, 28

Myosoton aquaticum, 33

Myosurus minimus, 84

"Myrica, 61

caroliniensis, 61

cerifera, 61

heterophylla, 61

pensylvanica, 61

Myriophyllum, 51-52

aquaticum, 51

"brasiliense, 51

*exalbescens, 52

heterophyllum, 51

humile, 51

pinnatum, 51

"proserpinacoides, 51

sibiricum, 52

spicatum, 52

tenellum, 52

verticillatum, 52

Myriopteris lanosa, 8

Nabalus, 22

albus, 22

altissimus, 22

autumnalis, 22

crepidineus, 22 
Nabalus (continued) serpentarius, 22 trifoliolatus, 22

Najas, 52

canadensis, 52

flexilis, 52

gracillima, 52

guadalupensis ssp.

guadalupensis, 52

minor, 52

Nama, 28, 52

hispida, 28

*quadrivalvis, 52

Nandina domestica, 27

Napaea dioica, 59

Narcissus, 12

jonquilla, 12

poeticus, 12

pseudonarcissus, 12

Narthecium americanum, 61

Nasturtium officinale, 30

*Negundo negundo, 90

Nekemias arborea, 94, 116

Nelumbo, 61, 108

lutea, 61 nucifera, 61, 108

*Nemopanthus mucronatus, 15

Nemophila, 28

aphylla, 28

"microcalyx, 28

Neobeckia aquatica, 30

*Neocleome spinosa, 35

Neottia, 64

bifolia, 64

cordata, 64

smallii, 64

Nepeta cataria, 56

Nicandra physalodes, 92

Nicotiana, 92, 115

alata, 92

glauca, 92

longiflora, 92, 115

obtusifolia, 92

plumbaginifolia, 92

tabacum, 92

"trigonophylla, 92

Nigella damascena, 84

*Norta altissima, 30

"Nothoholcus lanatus, 75

Nuphar, 61

advena, 61

"lutea ssp. advena, 61

"lutea ssp. variegata, 61 variegata, 61

"Nuttallanthus canadensis, 67

Nymphaea, 61, 108

odorata ssp. odorata, 61

odorata ssp. tuberosa, 61, 108

*tuberosa, 61, 108

Nymphoides, 60

aquatica, 60

cordata, 60 "lacunosa, 60

peltata, 60

Nyssa, 61

biflora, 61

sylvatica, 61

*sylvatica var. biflora, 61

Obolaria virginica, 51

Oclemena, 22

acuminata, 22

nemoralis, 22

Oenanthe, 13, 98

aquatica, 13, 98

javanica, 13

Oenothera, 63

argillicola, 63

*argillicola var. pubescens, 63

biennis, 63

"biennis var. austromontana, 63

"biennis var. canescens, 63

clelandii, 63

fruticosa, 63

"ruticosa ssp. glauca, 63

*fruticosa ssp. microcarpa, 63

*fruticose var. subglobosa, 63

grandis, 63

humifusa, 63

laciniata, 63

*laciniata var. grandiflora, 63

nutans, 63

oakesiana, 63

parviflora, 63

perennis, 63

tetragona, 63

"tetragona var. fraseri, 63

villosa ssp. villosa, 63

Oldenlandia uniflora, 89

Oligoneuron, 22

*grandiflorum, 22

rigidum var. rigidum, 22

*Onagra biennis, 63

Onoclea sensibilis, 7

Ononis, 48

campestris, 48

repens, 48

*spinosa, 48

Onopordum acanthium, 22

*Onosmodium, 28, 101

hispidissimum, 28

molle var. hispidissimum, 28, 101

virginianum, 28

Ophioglossum, 7 pusillum, 7

*pycnostichum, 7

vulgatum, 7

*vulgatum var. pycnostichum, 7
Oplismenus, 76

"hirtellus ssp. undulatifolius, 76 undulatifolius, 76

Opuntia, 31

cespitosa, 31

*compressa, 31

humifusa var. humifusa, 31

mesacantha ssp. mesacantha, 31

Orbexilum, 48 "pedunculatum, 48 psoralioides, 48

*Orchis spectabilis, 63

Oreojuncus trifidus, 54

Origanum vulgare, 56

Ornithogalum, 16

nutans, 16

umbellatum, 16

Orobanche, 65

minor, 65

*uniflora, 65

Orontium aquaticum, 15

Orthilia secunda, 44

Orthocarpus bracteosus, 65

Oryzopsis, 76, 77

asperifolia, 76

*miliacea, 77

*racemosa, 77

Osmanthus heterophyllus, 62

Osmorhiza, 13

claytonii, 13

longistylis, 13

Osmunda, 7

"cinnamomea var. cinnamomea, 7

*innamomea var. glanduosa, 7

*claytoniana, 7

regalis var. spectabilis, 7

Osmundastrum, 7

cinnamomeum var. cinnamomeum, 7

cinnamomeum var. glandulosum, 7

*Osmundopteris virginiana, 7

Ostrya virginiana, 27

Oxalis, 65

colorea, 65

corniculata, 65

*ymosa, 65

dillenii, 65

*dillenii ssp. filipes, 65

"europaea, 65

florida, 65

"florida var. filipes, 65

*fontana, 65

grandis, 65

montana, 65

stricta, 65

"stricta, 65

violacea, 65
*Oxybasis, 11, 98 rubra, 11, 98 urbica, 11, 98

*Oxycoccus, 45 macrocarpus, 45 ovalifolius, 45

Oxydendrum arboreum, 44

Oxypolis, 13, 14

"canbyi, 14

rigidior, 13

"rigidior var. longifolia, 13

"turgida, 13

"Pachistima canbyi, 34

Pachysandra, 31, 101 procumbens, 31, 101 terminalis, 31, 101

Packera, 22-23, 100

anonyma, 22

antennariifolia, 22

aurea, 22

crawfordii, 23

glabella, 23, 100

obovata, 23

paupercula, 23

tomentosa, 23

Palustricodon aparinoides, 31

Panax, 16

quinquefolius, 16

trifolius, 16

"Panicularia, 74, 75

acutiflora, 74

nervata, 75

septentrionalis, 75

Panicum, 70, 71, 72, 73, 75,

$76,78,79$

*aciculare, 71

*acuminatum var. acuminatum, 71

"acuminatum var. columbianum, 71

"acuminatum var. densiflorum, 72

"acuminatum var. fasciculatum, 72

*acuminatum var. implicatum, 72

*acuminatum var. leucothrix, 72

*acuminatum var. lindheimeri, 72

"acuminatum var. longiligulatum, 72

*acuminatum var. thurowii, 71

*acuminatum var. unciphyllum, 72

*agrostoides, 71

amarulum, 76

amarum, 76

"amarum ssp. amarulum, 76

*anceps ssp. rhizomatum, 70 
"anceps var. anceps, 70

"angustifolium, 71

*arenicoloides, 71

"auburne, 71

*barbulatum, 72

*bicknellii, 71

"boscii, 71

capillare, 76

*capillare var. agreste, 76

*capillare var. campestre, 76

*capillare var. occidentale, 76

*capillare var. sylvaticum, 76

"chrysopsidifolium, 72

*clandestinum, 71

*colonum, 73

*columbianum, 71

"commutatum, 71

*commutatum var. joorii, 71

*condensum, 71

*crus-galli, 73

*depauperatum, 72

dichotomiflorum var. dichotomiflorum, 76

*dichotomiflorum var. geniculatum, 76

*dichotomum var. dichotomum, 72

*dichotomum var. lucidum, 72

*dichotomum var. roanokense, 72

*dichotomum var. yadkinense, 73

*elongatum var. ramosius, 71

*ensifolium, 72

"equilaterale, 71

*fasciculatum, 72 flexile, 76

gattingeri, 76

"gibbum, 78

"haemacarpon, 73

*hemitomon, 75

"buachucae, 72

*implicatum, 72

*joorii, 71

*lanuginosum, 72

*latifolium, 72

"laxiflorum, 72

*leucothrix, 72

*lindheimeri, 72

"linearifolium, 72

*longifolium var. combsii, 70

*longifolium var. longifolium, 71

*longiligulatum, 72

*lucidum, 72

"meridionale, 72

miliaceum, 76 "mutabile, 71

*oligosanthes, 72

*ovale, 72

"ovale var. villosum, 73

*ovinum, 71

paludosum, 76

"parvispiculum, 72

"paucipilum, 72

philadelphicum, 76

"philadelphicum ssp. gattingeri, 76

"polyanthes, 72

*proliferum, 76

"prostratum, 79

*psilopodium, 76

"pubescens, 72

*purpurascens, 79

"pyriforme, 72

"ramosum, 79

"ravenelii, 72

"reptans, 79

*rigidulum, 71

*rigidulum ssp. elongatum, 71

"rigidulum ssp. pubescens, 71

"rigidulum var. combsii, 70

*rigidulum var. condensum, 71

"rigidulum var. rigidulum, 71

"roanokense, 72

"scabriusculum, 72

"scoparium, 72

*sphaerocarpon, 72

"sphagnicola, 72

"spretum, 72

*striata, 75

sumatrense, 76

"tennesseense, 72

"tenue, 73

*texanum, 79

*thurowii, 71

*tsugetorum, 71

"tuckermanii, 76

"verrucosum, 75

*villosissimum, 73

virgatum, 76

*virgatum var. cubense, 76

"virgatum var. spissum, 76

*viscidum, 72

*walteri, 73

"werneri, 72

*wrightianum, 73

*xalapense, 72

*xanthospermum, 73

*yadkinense, 73

*Panicum, 110

anceps ssp. rhizomatum, 110

angustifolium, 110

arenicoloides, 110

dichotomum var. roanokense, 110 ovale, 110

roanokense, 110

*Panium, 71, 72, 110

boreale, 71,110

mattamuskeetense, 72

Papaver, 66, 109

argemone, 66

dubium, 66

nudicaule, 66, 109

orientale, 66, 109

rhoeas, 66

somniferum, 66, 109

"Papyrius papyriferus, 61

Parapholis incurva, 76

Parathelypteris noveboracensis, 8

Parietaria, 93, 115

floridana, 93, 115

judaica, 93, 115

pensylvanica, 93

Parnassia asarifolia, 34

Paronychia, 33, 102

argyrocoma, 33, 102

canadensis, 33

dichotoma, 33

fastigiata, 33

*fastigiata var. nuttallii, 33

*fastigiata var. paleacea, 33

*fastigiata var. pumila, 33

montana, 33

virginiana, 102

virginica var. virginica, 33

"Parosela floridana, 47

*Parsonsia petiolata, 58

Parthenium, 23, 100

auriculatum, 23, 100

"hispidum var. auriculatum, 23, 100

hysterophorus, 23

integrifolium, 23

Parthenocissus, 94, 116

"hirsuta, 94

inserta, 94

quinquefolia, 94

*quinquefolia var. vitacea, 94, 116

tricuspidata, 94, 116

*vitacea, 94, 116

Paspalum, 76-77, 111

boscianum, 76

*calvescens, 76

"ciliatifolium, 76

"ciliatifolium var. mublenbergii, 76

"ciliatifolium var. stramineum, 77

*circulare, 76

convexum, 76

"difforme, 76

dilatatum, 76

dissectum, 76

distichum, 76

floridanum, 76 "floridanum var. glabratum, 76

fluitans, 76

"giganteum, 76

*glabratum, 76

laeve, 76

*laeve var. circulare, 76

*laeve var. pilosum, 76

"longipilum, 76

*membranaceum, 76

orbiculatum, 76

"paspaloides, 76

pubiflorum var. glabrum, 76

"repens, 76

scrobiculatum, 76

setaceum var. ciliatifolium, 76

setaceum var. mublenbergii, 76

setaceum var. setaceum, 77 setaceum var. villosissimum, 77, 111

*stramineum, 77

Passiflora, 66

foetida, 66

"foetida var. isthmia, 66 incarnata, 66

lutea, 66

Pastinaca sativa, 13

Patis racemosa, 77

Paulownia tomentosa, 66

Paxistima canbyi, 34

Pedicularis, 65

canadensis, 65

lanceolata, 65

Pellaea, 8

atropurpurea, 8

glabella, 8

glabella ssp. glabella, 8

Peltandra virginica, 15

"Pennisetum, 70 alopecuroides, 70 glaucum, 70

Penstemon, 67, 109 alluviorum, 67, 109

"brevisepalus, 67 calycosus, 67, 109

canescens, 67

digitalis, 67

birsutus, 67

laevigatus, 67

pallidus, 67

"penstemon, 67

Penthorum sedoides, 66

"Peramium pubescens, 63

Perilla, 56

frutescens, 56

"frutescens var. crispa, 56

Persea, 57

palustris, 57

"pubescens, 57

Persicaria, 81, 112

amphibia, 81

"amphibia var. emersa, 81 
Persicaria (continued) arifolia, 81

careyi, 81, 112

chinensis, 81, 112

"densiflora, 81

extremiorientalis, 81

filiformis, 81

glabra, 81

bydropiper, 81

hydropiperoides, 81

lapathifolia, 81

longiseta, 81

*longistyla, 81

maculosa, 81

"mississippiensis, 81

*opelousana, 81

orientalis, 81

pensylvanica, 81

perfoliata, 81

"persicaria, 81

punctata, 81

robustior, 81

sagittata, 81

setacea, 81

virginiana, 81

Petrorhagia prolifera, 33

Petroselinum crispum, 13, 98

Petunia, 91, 92

xatkinsiana, 92

*axillaris, 92

integrifolia, 92

"parviflora, 91

*Peucedanum ostruthium, 13

Phacelia, 28, 101

*bicknellii, 28

bipinnatifida, 28, 101

"boykinii, 28

"brevistyla, 28, 101

covillei, 28, 101

dubia, 28

purshii, 28

ranunculacea, 28, 101

*Phaethusa occidentalis, 26

Phalaris, 77, 111

arundinacea, 77

canariensis, 77

caroliniana, 77, 111

minor, 77

paradoxa, 77

*Pharbitis, 36

barbigera, 36

hederacea, 36

purpurea, 36

Phaseolus, 48, 49, 50

"helvolus, 49

"mungo, 50

polystachios, 48

"umbellatus, 49

vulgaris, 48

Phegopteris, 8

connectilis, 8

hexagonoptera, 8

Phellodendron, 89

amurense, 89

*japonicum, 89
Phemeranthus teretifolius, 92

"Phenianthus sempervirens, 32

Philadelphus, 52

coronarius, 52

"gloriosus, 52

"grandiflorus, 52

birsutus, 52

inodorus, 52

"intectus, 52

pubescens, 52

*Philotria, 52

canadensis, 52

densa, 52

Pbleum, 77

pratense, 77

subulatum, 77

Phlox, 80, 112

amplifolia, 80

carolina, 80, 112

"carolina ssp. angusta, 80, 112

"carolina ssp. turritella, 80, 112

divaricata var. divaricate, 80

*divaricata var. laphamii, 80

drummondii, 80

glaberrima, 80

"glaberrima ssp. interior, 80

"glaberrima ssp. triflora, 80

*latifolia, 80, 112

maculata, 80

"maculata ssp. pyramidalis, 80

ovata, 80,112

paniculata, 80

pilosa, 80

"pilosa ssp. detonsa, 80

"reptans, 80

revoluta, 112

stolonifera, 80

subulata, 80

"subulata var. brittonii, 80

Phoenix dactylifera, 16

Phoradendron, 90

*flavescens, 90

leucarpum, 90

*serotinum, 90

Photinia, 85, 87

*floribunda, 85

*melanocarpa, 85

*pyrifolia, 85

villosa, 87

Phragmites, 77

australis ssp. americanus, 77

australis ssp. australis, 77

*communis, 77

*phragmites, 77

Phryma leptostachya, 66

Phyla, 93

*incisa, 93

lanceolata, 93

nodiflora, 93
Phyllanthus caroliniensis ssp. caroliniensis, 66

"Phyllitis scolopendrium var. americana, 6

Phyllostachys, 77, 111

aurea, 77

aureosulcata, 77

bambusoides, 77

flexuosa, 77, 111

nigra, 77

Physalis, 92, 115

alkekengi, 92

"ambigua, 92

angulata, 92

"barbadensis, 92

*floridana, 92

grisea, 92, 115

heterophylla, 92

*heterophylla var. nyctaginea, 92

*intermedia, 92

longifolia var. subglabrata, 92

*monticola, 92

"nyctaginea, 92

philadelphica, 92

*pruinosa, 92, 115

pubescens var. integrifolia, 92

pubescens var. pubescens, 92

*sinuata, 92

*subglabrata, 92

virginiana var. virginiana, 92

*Physalodes physalodes, 92

Physocarpus opulifolius, 87

Physostegia, 56, 106

purpurea, 56, 106

virginiana ssp. virginiana, 56

Phytolacca, 66

americana, 66

bogotensis, 66

*decandra, 66 icosandra, 66

Picea, 8, 97

abies, 8, 97

glauca, 8, 97

"glauca var. densata, 8,97

pungens, 8, 97

rubens, 8

Picris, 21, 23, 100

"echioides, 21, 100

hieracioides, 23

Pieris floribunda, 44

Pilea, 93

fontana, 93

pumila, 93

"Pilosella, 21

caespitosa, 21

officinarum, 21

piloselloides, 21

Pilularia americana, 7
Pimpinella saxifraga, 13

Pinellia ternata, 15

Pinus, 8-9

echinata, 8

nigra, 8

palustris, 8

pungens, 8

resinosa, 9

rigida, 9

serotina, 9

strobus, 9

sylvestris var. sylvestris, 9

taeda, 9

thunbergiana, 9

virginiana, 9

Piptatherum, 77

miliaceum, 77

*racemosum, 77

Piptochaetium avenaceum, 77

Pistia stratiotes, 15

Pisum, 48

fulvum, 48

sativum, 48

Pityopsis graminifolia var. latifolia, 23

Planodes virginicum, 30

Plantago, 67, 109

aristata, 67

cordata, 67

heterophylla, 67, 109

"bybrida, 67

lanceolata, 67

major, 67

maritima, 67, 109

*maritima var juncoides, 67, 109

pusilla, 67

rugelii, 67

virginica, 67

wrightiana, 67, 109

Platanthera, 64, 108-109

blephariglottis, 64

ciliaris, 64

clavellata, 64

cristata, 64

"fissa, 64

flava var. flava, 64

flava var. herbiola, 64

grandiflora, 64

integra, 64, 108

lacera, 64

macrophylla, 64, 109

nivea, 64, 109

orbiculata, 64

peramoena, 64

psycodes, 64

shriveri, 64

Platanus occidentalis, 68

Pleioblastus, 77

fortunei, 77

*pygmaeus, 77

Pleopeltis, 7

michauxiana, 7

*polypodioides ssp. michauxiana, 7 
Pluchea, 23

camphorata, 23

foetida var. foetida, 23

odorata var. odorata, 23

*odorata var. succulenta, 23

*petiolata, 23

*purpurascens, 23

*purpurascens var. succulenta, 23

*tenuifolia, 23

Poa, 77, 102, 111

alsodes, 77

annua, 77

autumnalis, 77

bulbosa var. vivipara, 77

chapmaniana, 77, 111

compressa, 77

cuspidata, 77

iconia var. iconia, 77

languida, 77, 102, 111

nemoralis, 77

palustris, 77

pratensis ssp. angustifolia, 77

pratensis ssp. pratensis, 77

saltuensis, 77, 111

sylvestris, 77

trivialis ssp. sylvicola, 77

Podophyllum peltatum, 27

Podostemum, 79

*abrotanoides, 79

ceratophyllum, 79

Pogonia, 63, 64

"divaricata, 63

ophioglossoides, 64

*Poinsettia, 45

cyathophora, 45

dentata, 45

Polanisia, 35

dodecandra ssp. dodecandra, 35

*trachysperma, 35

Polemonium, 80 reptans var. reptans, 80 vanbruntiae, 80

*Polianthes virginica, 16, 99

Polycnemum majus, 11

*Polycodium candicans, 45

Polygala, 80, 112

"ambigua, 80

brevifolia, 80, 112

cruciata, 80

"cruciata var. aquilonia, 80

curtissii, 80

cymosa, 80, 112

*harperi, 80

incarnata, 80

lutea, 80

mariana, 80

nuttallii, 80

paniculata, 80, 112

"pauciflora, 80

polygama, 80

"pretzii, 80 ramosa, 80,112

"ramosior, 80

sanguinea, 80

senega var. latifolia, 80

senega var. senega, 80

verticillata, 80

*verticillata var. isocycla, 80

*verticillate var. ambigua, 80

*viridescens, 80

Polygaloides pancifolia, 80

Polygonatum, 16

biflorum var. biflorum, 16

biflorum var. commutatum, 16

*canaliculatum, 16

*commutatum, 16

pubescens, 16

Polygonella articulata, 81

Polygonum, 80, 81, 112

"amphibium, 81

"arenastrum, 81

"arifolium, 81

"articulatum, 81

aviculare, 81

*aviculare ssp. bellardii, 81

*aviculare ssp. depressum, 81

"baldschuanicum, 80, 112

"bellardii, 81

*careyi, 81, 112

"cespitosum var. longisetum, 81

"cilinode, 80

coccineum, 81

*convolvulus, 80

"cuspidatum, 81

*densiflorum, 81

douglasii, 81

*dubium, 81

erectum, 81

"exsertum, 81

glaucum, 81

"hydropiper, 81

"hydropiperoides, 81

*incarnatum, 81

"lapathifolia, 81

"maculatum, 81

"maritimum, 81

mublenbergii, 81

*opelousanum, 81

*orientale, 81

*pensylvanicum, 81

"pensylvanicum var. rosaeflorum, 81

*perfoliatum, 81

*persicaria, 81

"portoricense, 81

prolificum, 81

"punctatum, 81

"puritanorum, 81

ramosissimum, 81

"ramosissimum var. prolificum, 81 "robustius, 81

"sachalinense, 81

"sagittatum, 81

"scandens var. cristatum, 80

"scandens var. dumetorum, 80, 112

"scandens var. scandens, 80

"setaceum, 81

tenue, 81

*virginianum, 81

Polymnia, 23, 24

canadensis, 23

*canadensis var. radiata, 23

"radiata, 23

*uvedalia, 24

Polypodium, 7

appalachianum, 7

*polypodioides, 7

virginianum, 7

*vulgare, 7

Polypogon monspeliensis, 77

Polypremum procumbens, 93

Polystichum acrostichoides, 6

Poncirus trifoliata, 89

Pontederia, 82

cordata, 82

crassipes, 82

*lanceolata, 82

Populus, 89-90

alba, 89

balsamifera, 89

deltoides, 90

grandidentata, 90

heterophylla, 90

nigra, 90

"nigra var. italica, 90

tremuloides, 90

*Porteranthus, 86, 114

stipulatus, 86, 114

trifoliatus, 86

Portulaca, 82

amilis, 82

grandiflora, 82

oleracea, 82

Potamogeton, 82, 112-113

"americanus, 82

amplifolius, 82

"angustifolius, 82

"berchtoldii var.

lacunatus, 82

bicupulatus, 82

"capillaceus, 82

crispus, 82, 113

diversifolius, 82, 113

epibydrus, 82

"fluitans, 82

foliosus, 82

gramineus, 82

"heterophyllus, 82

illinoensis, 82

"lonchites, 82

"lucens, 82

natans, 82 nodosus, 82

obtusifolius, 82, 112

*pectinatus, 82

perfoliatus, 82, 113

"perfoliatus var. bupleuroides, 82

praelongus, 82, 112

pulcher, 82

pusillus ssp. pusillus, 82

pusillus ssp. tenuissimus, 82

"pusillus var. minor, 82

richardsonii, 82, 113

robbinsii, 82

spirillus, 82, 113

vaseyi, 82

zosteriformis, 82

Potentilla, 85, 87, 88, 113

*anserina, 85, 113

argentea, 87

arguta, 87

canadensis var. canadensis, 87

*canadensis var. villosissima, 87

*caroliniana, 87

indica, 87

intermedia, 87

*monspeliensis, 87

norvegica, 87

*norvegica ssp. monspeliensis, 87

"pumila, 87

recta, 87

reptans, 87

rivalis, 87

simplex, 87

*tridentata, 88

Poterium, 87

annuum, 87

sanguisorba ssp. muricatum, 87

*Prenanthes, 22

alba, 22

altissima, 22

autumnalis, 22

crepidinea, 22

serpentaria, 22

trifoliolata, 22

Primula, 83

meadia, 83

veris, 83

"Prionopsis ciliatus, 20

Proboscidea lonisianica, 59

Prosartes, 58, 107

lanuginosa, 58

maculata, 58, 107

Proserpinaca, 52

*intermedia, 52

palustris, 52

*palustris var. crebra, 52 pectinata, 52

Prunella, 56

laciniata, 56

vulgaris ssp. lanceolata, 56 vulgaris ssp. vulgaris, 56 
Prunus, 87

alleghaniensis, 87

americana, 87

angustifolia, 87

avium, 87

cerasus, 87

domestica, 87

"domestica var. insititia, 87

hortulana, 87

*insititia, 87

mahaleb, 87

maritima, 87

"munsoniana, 87

pensylvanica, 87

persica, 87

pumila var. susquehanae, 87

rivularis, 87

serotina, 87

*serotina var. neomontana, 87

spinosa, 87

*susquehanae, 87

tomentosa, 87

virginiana, 87

Pseudognaphalium, 23

belleri, 23

obtusifolium, 23

Pseudolycopodiella caroliniana, 5

*Pseudolysimachion longifolium, 68

Pseudosasa japonica, 77

"Pseudotaenidia montana, 14

"Psilocarya, 42

nitens, 42

scirpoides, 42

*Psoralea psoraloides, 48

Ptelea trifoliata var. trifoliata, 89

Pteridium, 6

"aquilinum ssp. latiusculum, 6

"aquilinum ssp. pseudocaudatum, 6

aquilinum var.

latiusculum, 6

aquilinum var.

pseudocaudatum, 6

Pteris, 8

cretica, 8

multifida, 8

*Pteris, 6

aquilina, 6

aquilina var. pseudocaudata, 6

latiuscula, 6

Ptilimnium, 13

capillaceum, 13

*fluviatile, 13

nodosum, 13

Puccinellia, 77, 79

distans, 77

fasciculata, 77

"pallida, 79
Pueraria, 49

"lobata, 49

montana var. lobata, 49

"thunbergiana, 49

Pulicaria dysenterica, 23

Pycnanthemum, 56, 106-107

clinopodioides, 56

flexuosum, 56, 106

incanum, 56, 107

*incanum var.

puberulum, 56

muticum, 56

"pilosum, 56

pycnanthemoides, 56, 106-107

setosum, 56

tenuifolium, 56

torreyi, 56

verticillatum, 56

*verticillatum var. pilosum, 56

virginianum, 56

*Pycnodoria cretica, 8

*Pylostachya lutea, 80

Pyracantha coccinea, 87

Pyrola, 44

americana, 44

chlorantha, 44

elliptica, 44

"rotundifolia var. americana, 44

*secunda, 44

"virens var. convoluta, 44

"virens var. virens, 44

Pyrrhopappus carolinianus, 23

Pyrus, 85, 86, 87, 88

*americana, 88

*angustifolia, 86

*arbutifolia, 85

calleryana, 87

communis, 87

*coronaria, 86

*floribunda, 85

"malus, 87

"melanocarpa, 85

"pumila, 87

pyrifolia, 87

*sieboldii, 87

"Quamoclit coccinea, 36

Quercus, 50, 105

acutissima, 50

"agodifolia, 50

alba, 50

"alcata var. pagodifolia, 50

"axima, 50

bicolor, 50

*chneckii, 50

coccinea, 50

*cuminata, 50

falcata, 50

*igitata, 50

ilicifolia, 50 imbricaria, 50

*inor, 50

laurifolia, 50, 105

lyrata, 50

macrocarpa, 50

marilandica, 50

michauxii, 50

montana, 50

mublenbergii, 50

nigra, 50

*occinea var. tuberculata, 50

pagoda, 50

palustris, 50

phellos, 50

prinoides, 50

*rinoides var. acuminata, 50

*rinus, 50

rubra, 50

shumardii, 50

stellata, 50

"ubra var. borealis, 50

velutina, 50

*Radicula, 30

palustris, 30

sessiliflora, 30

sylvestris, 30

Ranunculus, 84, 113

abortivus, 84

acris, 84

allegheniensis, 84

ambigens, 84

*aquatilis, 84, 113

arvensis, 84, 113

bulbosus, 84

caricetorum, 84

*carolinianus, 84

"delphiniifolius, 84

fascicularis, 84

"ficaria ssp. calthifolius, 84

*ficaria var. bulbifera, 84

flabellaris, 84

hederaceus, 84

*hispidus var.

caricetorum, 84

hispidus var. hispidus, 84

hispidus var. nitidus, 84

laxicaulis, 84

"lindheimeri, 84

micranthus, 84

*oblongifolius, 84

*obtusiusculus, 84

*palmatus, 84

parviflorus, 84

"parvulus, 84

pensylvanicus, 84

pusillus, 84

"pusillus var. lindheimeri, 84

recurvatus, 84

repens, 84 sardous, 84

sceleratus var. sceleratus, 84

*septentrionalis, 84

trichophyllus var. trichophyllus, 84, 113

Raphanus, 30

raphanistrum, 30

sativus, 30

Reseda, 85

lutea, 85

luteola, 85

Reynoutria, 81

japonica, 81

sachalinensis, 81

Rhamnus, 85, 113

alnifolia, 85

"caroliniana, 85

cathartica, 85, 113

davurica, 85

*frangula, 85

lanceolata ssp. lanceolata, 85,113

Rhexia, 60, 108

aristosa, 60, 108

*lanceolata, 60

mariana, 60

"mariana var. ventricosa, 60

nashii, 60, 108

petiolata, 60, 108

"stricta, 60

ventricosa, 60

virginiana, 108

virginica, 60

*virginica var. purshii, 60

Rhododendron, 44-45, 104

arborescens, 44

atlanticum, 45

calendulaceum, 45, 104

canescens, 45, 104

maximum, 45

"nudiflorum, 45

periclymenoides, 45, 104

"pilosum, 44

prinophyllum, 45

"roseum, 45

viscosum, 45

*viscosum var. glaucum, 45

*viscosum var. serrulatum, 45

Rhodotypos scandens, 87

Rhus, 12

aromatica var. aromatica, 12

copallinum var. copallinum, 12

*copallinum var. latifolia, 12

* copallinum var. leucantha, 12

glabra, 12

"hirta, 12

*radicans, 12 
*toxicodendron, 12

"trilobata, 12

typhina, 12

*vernix, 12

*Rhynchelytrum repens, 75

Rhynchosia tomentosa var. tomentosa, 49

Rhynchospora, 42, 104 alba, 42

"axillaris, 42 capillacea, 42, 104 capitellata, 42

cephalantha, 42

"cephalantha var. microcephala, 42

chalarocephala, 42

corniculata, 42

"cymosa, 42

*cymosa var. globularis, 42

filifolia, 42, 104

fusca, 42

globularis var. globularis, 42

"globularis var. recognita, 42

glomerata, 42

"glomerata var. paniculata, 42

gracilenta, 42

harperi, 42, 104

inexpansa, 42

inundata, 42

macrostachya, 42

microcephala, 42

nitens, 42

oligantha, 42

pallida, 42

rariflora, 42

recognita, 42

scirpoides, 42

torreyana, 42

Ribes, 51, 106

americanum, 51

aureum, 51

cynosbati, 51

glandulosum, 51

hirtellum, 51, 106

missouriense, 51

nigrum, 51, 106

rotundifolium, 51

rubrum, 51

*sativum, 51

uva-crispa, 51

Richardia scabra, 89

Ricinus communis, 46

*Ridan alternifolia, 26

Ripariosida hermaphrodita, 59

*Ripidium ravennae, 79

Robinia, 49

"grandiflora, 49

hispida, 49

*hispida var. rosea, 49

"pallida, 49

pseudoacacia, 49 "speciosa, 49

viscosa, 49

Rorippa, 30

*islandica var. fernaldiana, 30

*islandica var. hispida, 30

"nasturtium, 30

"nasturtium-aquaticum, 30

palustris, 30

*palustris var. hispida, 30

sessiliflora, 30

sinuata, 30

sylvestris, 30

Rosa, 87, 114

blanda, 87

bracteata, 87

canina, 87

carolina var. carolina, 87

eglanteria, 87

*floridana, 87

gallica, 87

"bumilis, 87

*lancifolia, 87

luciae, 87, 114

"lyonii, 87

micrantha, 87

multiflora, 87

palustris, 87

"rubiginosa, 87

rugosa, 87

"serrulata, 87

setigera, 87

virginiana, 87

Rostraria cristata, 77

Rotala ramosior, 58

"Rubacer odoratus, 88

Rubus, 86, 88, 114

"aboriginum, 88

"adjacens, 88

allegheniensis, 88

"alumnus, 88

"andrewsianus, 88

"argutus, 88

"argutus var. floridus, 88

"baileyanus, 88

"betulifolius, 88

bifrons, 88

canadensis, 88

*centralis, 88

*continentalis, 88, 114

cuneifolius, 88

"curtipes, 88

*davisiorum, 88

*decar, 88

*defectionsis, 88

*depavitus, 88

*enslenii, 88

*fecundus, 88

flagellaris, 88

"flavinanus, 88

"floridus, 88

*frondosus, 88

"grimesii, 88

"heterophyllus, 88 bispidus, 88

idaeus var. strigosus, 88

"immanus, 88

"invisus, 88

"ithacanus, 88

laciniatus, 88

*laudatus, 88

*leviculus, 88

"longii, 88

"michiganensis, 88

"missouricus, 88

"montensis, 88

*multifer, 88

"nigrobaccus, 88

"notatus, 88

*obvius, 88

occidentalis, 88

odoratus, 88

*originalis, 88

*ostryigolius, 88

"paganus, 88

pascuus, 88

pensilvanicus, 88

"pervarius, 88

"philadelphicus, 88

phoenicolasius, 88

"plicatifolius, 88

"probabilis, 88

"provincialis, 88

pubescens, 88

"racemiger, 88

"recurvans, 88

"recurvicaulis, 88

"repens, 86

"rhodophyllus, 88

"roribaccus, 88

"rosa, 88

"rosarius, 88

"rubrisetus, 88, 114

"scambia, 88

setosus, 88

*steelei, 88

"suus, 88

trivialis, 88, 114

Rudbeckia, 23

"acuminata, 23

*amplectens, 23

"brittonii, 23

*digitata, 23

"foliosa, 23

fulgida var. fulgida, 23

hirta var. hirta, 23

hirta var. pulcherrima, 23

laciniata var. bipinnata, 23

"laciniata var. digitata, 23

laciniata var. humilis, 23

laciniata var. laciniata, 23

*longipes, 23

*monticola, 23

*sericea, 23

"serotina, 23

"spathulata, 23 "tenax, 23

triloba var. triloba, 23

"truncata, 23

Ruellia, 9

caroliniensis, 9

"ciliosa var. bybrida, 9

humilis, 9

*parviflora, 9

"pedunculata, 9

purshiana, 9

strepens, 9

*Rufacer, 90

carolinianum, 90

rubrum, 90

Rumex, 81-82, 112

acetosella, 81

altissimus, 81

crispus, 81

*floridanus, 82

fueginus, 81, 112

hastatulus, 81

"maritimus, 81, 112

obtusifolius, 82

patientia, 82

pulcher, 82

sanguineus, 82, 112

verticillatus, 82

Ruppia maritima, 89

Ruta graveolens, 89, 114

*Rytilix granularis, 75

Sabatia, 51

angularis, 51

campanulata, 51

difformis, 51

dodecandra, 51

stellaris, 51

*Sabina virginiana, 8

"Sabulina, 33

caroliniana, 33

stricta, 33

*Saccharodendron, 90

nigrum, 90

saccharum, 90

Saccharum, 77-78, 79, 111

alopecuroides, 77

baldwinii, 77

brevibarbe var. brevibarbe, 78, 111

brevibarbe var. contortum, 78

coarctatum, 78

*contortum, 78

giganteum, 78

"ravennae, 79

Sacciolepis striata, 78

Sagina, 33

apetala, 33

decumbens ssp. decumbens, 33

*decumbens var. smithii, 33

procumbens, 33 
Sagittaria, 10, 98

australis, 10

brevirostra, 10, 98

calycina, 10

"calycina var. spongiosa, 10

*cycloptera, 10

engelmanniana, 10

*falcata, 10

filiformis, 10, 98

graminea, 10

lancifolia ssp. media, 10

"lancifolia var. falcata, 10

latifolia var. latifolia, 10

latifolia var. pubescens, 10

*longirostra, 10

*longirostra var. australis, 10

*montevidensis ssp. calycina, 10

*montevidensis ssp. spongiosa, 10

*obtusa, 10

"pubescens, 10

rigida, 10

spathulata, 10

"stagnorum, 10, 98

subulata, 10

"subulata var. gracillima, 10, 98

teres, 10, 98

*viscosa, 10

Salicornia, 11

ambigua, 11

bigelovii, 11

*depressa, 11

"europaea, 11

virginica, 11

Salix, 90, 114

alba, 90

"alba var. vitellina, 90

amygdaloides, 90, 114

babylonica, 90

bebbiana, 90

caprea, 90, 114

caroliniana, 90

cinerea, 90

*cordata, 90

discolor, 90

elaeagnos, 90, 114

eriocephala, 90

exigua var. sericans, 90

fragilis, 90

humilis, 90

"humilis var. microphylla, 90

"humilis var. tristis, 90

*incana, 114

*interior, 90

*longipes, 90

lucida, 90

"marginata, 90

nigra, 90 occidentalis, 90

pentandra, 90

purpurea, 90

"rigida, 90

sericea, 90

triandra, 90, 114

"tristis, 90

*wardii, 90

Salsola, 12

kali ssp. kali, 12

kali ssp. pontica, 12

"kali var. tenuifolia, 12

"pestifer, 12

tragus, 12

Salvia, 56, 107

lyrata, 56

officinalis, 56

pratensis, 56

reflexa, 56

urticifolia, 56

verbenacea, 56

verticillata, 56, 107

Salvinia, 8, 97

minima, 8, 97

*rotundifolia, 8, 97

Sambucus, 9

canadensis, 9

"nigra ssp. canadensis, 9

"pubens, 9

racemosa var. pubens, 9

*simpsonii, 9

Samolus, 83

"floribundus, 83

"parviflorus, 83

valerandi, 83

*valerandi ssp.

parviflorus, 83

Sanguinaria canadensis, 66

Sanguisorba, 87, 88

"annua, 87

canadensis, 88

"minor, 87

Sanicula, 13

canadensis var. canadensis, 13

"floridana, 13

*gregaria, 13

marilandica, 13

odorata, 13

trifoliata, 13

Sanvitalia, 23

*angustifolia, 23

procumbens, 23

Saponaria, 33, 34

officinalis, 33

"porrigens, 33

*vaccaria, 34

"Saracha procumbens, 91

"Sarcocornia, 11 ambigua, 11

perennis, 11

"Sarothra, 52, 53

drummondii, 52

gentianoides, 53
Sarracenia, 90

flava, 90

purpurea, 90

"purpurea var. venosa, 90

Sassafras, 57

albidum, 57

*albidum var. molle, 57

*Satureja, 55, 106

acinos, 55, 106

calamintha var. nepeta, 55

Saururus cernuus, 90

"Saxifraga, 91, 115

michauxii, 91, 115

micranthidifolia, 91

pensylvanica, 91

virginiensis, 91

Scandix pecten-veneris, 13, 98

Sceptridium, 7, 97

biternatum, 7

dissectum, 7

jenmanii, 7, 97

multifidum, 7

*oneidense, 7

*Schedonorus, 75

arundinaceus, 75

pratensis, 75

Schenkia spicata, 51

Schizachne purpurascens, 78

Schizachyrium, 78

littorale, 78

scoparium var.

$$
\text { scoparium, } 78
$$

Schkubria, 23

pinnata, 23

*wislizenii, 23

Schoenoplectiella, 42-43

mucronata, 42

purshiana var. purshiana, 42

purshiana var. williamsii, 43

smithii var. setosa, 43

Schoenoplectus, 37, 42, 43, 104

acutus var. acutus, 43, 104

americanus, 43

etuberculatus, 43

"fluviatilis, 37

"mucronatus, 42

"novae-angliae, 37

pungens var. pungens, 43

"purshianus, 42

"robustus, 37

*smithii var. setosus, 43

subterminalis, 43

tabernaemontani, 43, 104

torreyi, 43

Schwalbea, 65

americana, 65

*australis, 65

Scirpus, 37, 41, 42, 43, 103, 104

"americanus, 43

ancistrochaetus, 43

atrovirens, 43

"atrovirens var. georgianus, 43 *cylindricus, 37

cyperinus, 43

"cyperinus var. eriophorum, 43

*cyperinus var. pelius, 43

*debilis, 42

"eriophorum, 43

"etuberculatus, 43

expansus, 43

*fluviatilis, 37

georgianus, 43

hattorianus, 43

"lacustris, 43

lineatus, 43, 104

"nanus, 41

*novae-angliae, 37

*olneyi, 43

"paludosus, 37, 103

pendulus, 43, 104

polyphyllus, 43

"pungens, 43

*purshianus, 42

*robustus, 37

"rubricosus, 43

*subterminalis, 43

"sylvaticus, 43

"tabernaemontani, 43

"validus, 43

*verecundus, 43

Scleranthus annuus, 33

Scleria, 43

minor, 43

mublenbergii, 43

"nitida, 43

oligantha, 43

pauciflora, 43

"pauciflora var. caroliniana, 43

reticularis, 43

"setacea, 43

"torreyana, 43

triglomerata, 43

*triglomerata var. minor, 43

verticillata, 43

Sclerochloa dura, 78

Sclerolepis uniflora, 23

Scrophularia, 91

lanceolata, 91

marilandica, 91

Scutellaria, 56, 107

*ambigua, 56

*campestris, 107

*cordifolia, 56

elliptica var. elliptica, 56

galericulata, 56

incana var. incana, 56

integrifolia, 56

lateriflora, 56

leonardii, 56

nervosa, 56

*ovalifolia, 56

ovata ssp. rugosa, 56

*ovata ssp. virginiana, 56 
"parvula var. missouriensis, 56 parvula var. parvula, 56, 107 *pilosa, 56 saxatilis, 56 serrata, 56

Secale cereale, 78

Securigera, 49

cretica, 49

varia, 49

Sedum, 36 acre, 36 glaucophyllum, 36 *purpureum, 36 sarmentosum, 36 *telephioides, 36 *telephium, 36 ternatum, 36

Selaginella, 5 apoda, 5 rupestris, 5

Senecio, 22, 23, 100

*anonymus, 22

"antennariifolius, 22

"aureus, 23

"aureus var. angustifolius, 22

bicolor, 23, 100

"cineraria, 23, 100

"crawfordii, 23

"earlei, 22

"glabellus, 23, 100

*obovatus, 23

"pauperculus, 23

*smallii, 22

suaveolens, 23

"tomentosus, 23 vulgaris, 23

Senna, 49, 105 baubinioides, 49 hebecarpa, 49 italica, 49 marilandica, 49 obtusifolia, 49 occidentalis, 49, 105

Sericocarpus, 24 asteroids, 24 linifolius, 24

"Sesban exaltatus, 49

Sesbania, 49

"exaltata, 49

herbacea, 49

*macrocarpa, 49

Seseli libanotis, 13

Sesuvium maritimum, 9

Setaria, 78

faberi, 78

"geniculata, 78

"glauca, 78

grisebachii, 78

italica, 78

*lutescens, 78

magna, 78

parviflora, 78 pumila, 78

*pumila ssp. pallidefusca, 78

verticillata, 78

"verticillata var. ambigua, 78 verticilliformis, 78 viridis, 78

Sherardia arvensis, 89

"Sibara virginica, 30

Sibbaldiopsis tridentata, 88

Sicyos angulatus, 36

Sida, 59

*angustifolia, 59

cordata, 59

"hermaphrodita, 59

"humilis var. veronicaefolia, 59

rhombifolia, 59

spinosa, 59

Sideroxylon lycioides, 90, 115

"Sieglingia purpurea, 79

Silene, 33-34, 102

*alba, 34

antirrhina, 33

armeria, 33

caroliniana ssp. pensylvanica, 33

chalcedonica, 33, 102

conica, 33, 102

conoidea, 33, 102

coronaria, 33

csereii, 33

dichotoma, 33

dioica, 33

drummondii, 33, 102

flos-cuculi, 34

latifolia, 34

"latifolia ssp. alba, 34

nivea, 34

noctiflora, 34

stellata, 34

virginica, 34, 102

vulgaris, 34

Silphium, 24, 100

asteriscus var. trifoliatum, 24

perfoliatum var.

connatum, 24, 100

perfoliatum var. perfoliatum, 24, 100

*trifoliatum, 24

Silybum marianum, 24

Simsia lagascaeformis, 24

Sinapis, 30

alba, 30

arvensis, 30

Sisymbrium, 30

altissimum, 30

loeselii, 30

"nasturtium-aquaticum, 30

officinale, 30

Sisyrinchium, 53

angustifolium, 53

arenicola, 53 atlanticum, 53

"graminoides, 53

montanum, 53

mucronatum, 53

"mucronatum var. atlanticum, 53

*Sitilias caroliniana, 23

Sium suave, 13

Smallanthus uvedalia, 24

"Smilacina, 16

racemosa, 16

stellata, 16

Smilax, 91

bona-nox, 91

ecirrata, 91

glauca, 91

*glauca var. leurophylla, 91

herbacea, 91

*herbacea var. pulverulenta, 91

*hispida, 91

laurifolia, 91

pseudochina, 91

pulverulenta, 91

rotundifolia, 91

tamnoides, 91

walteri, 91

Solanum, 92, 115

adscendens, 92

americanum, 92, 115

carolinense, 92

deflexum, 92

dulcamara, 92

elaeagnifolium, 92

emulans, 92

lycopersicum, 92

nigrum, 92

*nigrum var. villosum, 92

*nodiflorum, 92, 115

physalifolium, 92

"ptychanthum, 92, 115

rostratum, 92

"rubrum, 92

sarrachoides, 92, 115

*surattense, 92

torvum, 92

tuberosum, 92

villosum, 92

virginianum, 92

Solidago, 20, 22, 24-25, 100-101

altissima, 24

"angustifolia, 24

arguta var. arguta, 24

arguta var. boottii, 24, 100

arguta var. caroliniana, 24

*arguta var. harrisii, 24

bicolor, 24

caesia, 24

canadensis, 24

"canadensis var. hargeri, 24

*canadensis var. scabra, 24

"canadensis var. scabriuscula, 24 "celtidifolia, 24

*conferta, 24

curtisii, 24, 100

*edisoniana, 24

*elliottii, 24

erecta, 24

fistulosa, 24

flexicaulis, 24

gigantea, 24

"harperi, 24

harrisii, 24

"hirsutissima, 24

hispida, 24

juncea, 24

"lancifolia, 24, 100

latissimifolia, 24

*mexicana, 24

"mirabilis, 24

missouriensis, 24, 100

"neglecta, 25

nemoralis ssp. nemoralis, 24

*odora var. inodora, 24

odora var. odora, 24

patula var. patula, 24

"petiolata, 24

"pubens, 100

puberula var. puberula, 24

puberula var. pulverulenta, 24, 100

"pulverulenta, 24, 100

racemosa, 24

*rigida var. rigida, 22

roanensis, 24

"rugosa ssp. aspera, 24

rugosa var. aspera, 24

"rugosa var. celtidifolia, 24

rugosa var. rugosa, 24

rugosa var.

sphagnophila, 24, 100-101

"rugosa ssp. villosa, 24

rupestris, 24

sempervirens var. mexicana, 24, 101

sempervirens var. sempervirens, 24

*serotina, 24

"serotina var. gigantea, 24

*simplex var. racemosa, 24

speciosa var. speciosa, 24

squarrosa, 24

stricta, 24, 101

tarda, 25

"tenuifolia, 20

uliginosa, 25

ulmifolia var. ulmifolia, 25

*uniligulata, 25

Sonchus, 25

arvensis, 25

"arvensis var. glabrescens, 25

asper, 25

oleraceus, 25

uliginosus, 25 
*Sophia, 29 millefolia, 29

pinnata, 29

"Sophora japonica, 49

Sophronanthe pilosa, 68

Sorbus, 88

americana, 88 aucuparia, 88

Sorghastrum, 78 elliottii, 78 nutans, 78

Sorghum, 78

bicolor ssp. bicolor, 78

halepense, 78

*vulgare var. technicum, 78

Sparganium, 93

acaule, 93

americanum, 93

androcladum, 93

"chlorocarpum, 93

*emersum, 93

eurycarpum, 93

*Spartina, 78, 79

alterniflora, 78

cynosuroides, 78

patens, 79

patens var. caespitosa, 79

patens var. monogyna, 79

pectinate, 78

polystachya, 78

stricta var maritima, 78

*Specularia perfoliate, 31

Spergula, 34

arvensis, 34

morisonii, 34

Spergularia, 34

marina, 34

media, 34

rubra, 34

*salina, 34

Spermacoce glabra, 89

Spermolepis, 13, 98

inermis, 13, 98

"patens, 13, 98

Sphaeralcea angustifolia, 59

Sphenopholis, 78

intermedia, 78

nitida, 78

obtusata, 78

*obtusata var. major, 78

*obtusata var. pubescens, 78

pensylvanica, 78

Spigelia, 58, 107

"corymbosa, 58, 107

marilandica, 58, 107

Spinulum annotinum, 5

Spiraea, 88

alba, 88

corymbosa, 88

japonica, 88

latifolia, 88

prunifolia, 88 thunbergii, 88

tomentosa, 88

Spiranthes, 64, 109

arcisepala, 64

cernua, 64

"gracilis, 64

*grayi, 64

lacera var. gracilis, 64

lacera var. lacera, 64

laciniata, 64, 109

lucida, 64

ochroleuca, 64

odorata, 64

ovalis var. erostellata, 64

praecox, 64

romanzoffiana, 64

tuberosa, 64

vernalis, 64

*Spirea betulifolia var. corymbosa, 88

Spirodela, 15

polyrhiza, 15

"punctata, 15

Sporobolus, 78-79

alterniflorus, 78

"asper var. asper, 78

*berterianus, 78

clandestinus, 78

compositus var. compositus, 78

coromandelianus, 78

cynosuroides, 78

heterolepis, 78

indicus, 78

michauxianus, 78

neglectus, 79

"poiretii, 78

pumilus, 79

pyramidatus, 79

vaginiflorus var. vaginiflorus, 79

virginicus, 79

Stachys, 56-57, 107

*ambigua, 56

aspera, 56

byzantina, 56

clingmanii, 56, 107

cordata, 56, 107

eplingii, 56, 107

hispida, 56

"hyssopifolia var. ambigua, 56

byssopifolia var. byssopifolia, 57

latidens, 57, 107

longispicata, 57

nuttallii, 57, 107

palustris, 57

pilosa, 57, 107

*riddellii, 56, 107

tenuifolia, 57

"tenuifolia var. perlonga, 57

Staphylea trifolia, 92
"Steironema, 82, 83

ciliatum, 82

heterophyllum, 83

bybridum, 83

lanceolatum, 83

Stellaria, 33, 34, 102

alsine, 34

"aquatica, 33

borealis var. borealis, 34, 102

graminea, 34

longifolia, 34

longipes ssp. longipes, 34

media, 34

neglecta, 34, 102

pubera, 34

Stenanthium, 60

gramineum, 60

"gramineum var. micranthum, 60

*gramineum var. robustum, 60

leimanthoides, 60

*robustum, 60

"Stenophragma thalianum, 28

"Stenophyllus capillaris, 37

STERCULIACEAE, 108

"Sterculia platanifolia, 59, 108

"Stipa avenacea, 77

Streptopus, 58, 107

amplexifolius var. americanus, 58, 107

lanceolatus var. lanceolatus, 58

*roseus, 58

"Strophocaulos arvensis, 35

Strophostyles, 49

helvola, 49

leiosperma, 49

"pauciflora, 49

umbellata, 49

"umbellata var. paludigena, 49

Stuckenia pectinata, 82

Stylophorum diphyllum, 66

Stylosanthes, 49

biflora, 49

*biflora var. hispidissima, 49

"riparia, 49

"Stylypus vernus, 86

Styphnolobium japonicum, 49

Suaeda, 12

linearis, 12

maritima, 12

*Swida, 36

alternifolia, 36

amomum, 36

obliqua, 36

racemosa, 36

rugosa, 36

stolonifera, 36

stricta, 36

Symphoricarpos, 32

albus var. albus, 32

albus var. laevigatus, 32 occidentalis, 32

orbiculatus, 32

*symphoricarpos, 32

Symphyotrichum, 25-26

cordifolium, 25

depauperatum, 25

drummondii, 25

dumosum, 25

ericoides, 25

laeve var. concinnum, 25

laeve var. laeve, 25

lanceolatum var.

lanceolatum, 25

lanceolatum var. latifolium, 25

lateriflorum, 25

"lateriflorum var. horizontale, 25

*lateriflorus, 25

lowrieanum, 25

novae-angliae, 25

novi-belgii, 25

oblongifolium, 25

patens, 25

phlogifolium, 25

pilosum var. pilosum, 25

pilosum var. pringlei, 25

praealtum, 25

prenanthoides, 25

puniceum, 25

racemosum, 25

shortii, 25

subulatum, 25

tenuifolium, 25

undulatum, 26

urophyllum, 26

Symphytum, 28, 101

asperum, 28, 101

officinale, 28

Symplocarpus foetidus, 15

Symplocos tinctoria, 92

*Syndesmon

thalictroides, 84

"Synosma suaveolens, 23

Syringa vulgaris, 62

Taenidia, 13-14

integerrima, 13

montana, 14

*Tagetes, 19, 26

erecta, 26

minuta, 26

papposa, 19

pusilla, 26

*Talinum teretifolium, 92

*Tamala pubescens, 57

Tamarix, 92

parviflora, 92

ramosissima, 92

Tanacetum, 26

balsamita, 26

parthenium, 26

vulgare, 26 
Taraxacum, 26 erythrospermum, 26 *laevigatum, 26 officinale ssp. officinale, 26 "taraxacum, 26

*Tarenaya hassleriana, 35

Taxodium distichum, 8

Taxus canadensis, 9

Teesdalia nudicaulis, 31

Tephrosia, 49

purpurea, 49

spicata, 49

virginiana, 49

Tetradium daniellii, 89

Teucrium, 57

canadense, 57

*canadense var. occidentale, 57

*canadense var. virginicum, 57

laciniatum, 57

"littorale, 57

*occidentale, 57

*Thalesia uniflora, 65

Thalictrum, 84, 113

amphibolum, 84

coriaceum, 84

dasycarpum, 84, 113

dioicum, 84

macrostylum, 84, 113

"perelegans, 84

*polygamum, 84

pubescens, 84

"purpurascens, 84

"revolutum, 84

"steeleanum, 84

*subrotundum, 84, 113

thalictroides, 84

Thaspium, 14

barbinode, 14

"trifoliatum var. aureum, 14

"trifoliatum var. flavum, 14

trifoliatum var. trifoliatum, 14

Thelypteris, 8

"hexagonoptera, 8

*noveboracensis, 8

"palustris var. haleana, 8

palustris var. pubescens, 8

"phegopteris, 8

simulata, 8

*thelypteris, 8

Themeda, 79

arguens, 79

*frondosa, 79

Thermopsis, 49

"caroliniana, 49

villosa, 49

Thlaspi, 30, 31

alliaceum, 31

arvense, 31

"perfoliatum, 30
Thuja occidentalis, 8

Thymus, 57, 107

praecox arcticus, 57, 107

pulegioides, 57

"serpyllum, 57, 107

Thyrsanthella difformis, 14

Tiarella, 91

cordifolia, 91

"cordifolia var. collina, 91

*wherryi, 91

*Tiaridium indicum, 28

Tiedemannia canbyi, 14

Tilia, 59

americana, 59

*americana var.

heterophylla, 59

*australis, 59

*caroliniana, 59

cordata, 59

"eburnea, 59

*floridana, 59

"heterophylla, 59

*leucocarpa, 59

*michauxii, 59

*Tillaea aquatica, 36

*Tillaeastrum aquaticum, 36

Tillandsia usneoides, 31

Tipularia, 65

discolor, 65

*unifolia, 65

*Tissa, 34

marina, 34

rubra, 34

*Tofieldia, 93, 115 glutinosa, 93, 115 racemosa, 93

*Tomanthera auriculata, 65

Toona, 60, 108 ciliata, 60, 108 sinensis, 60

Torilis, 14

arvensis, 14

japonica, 14

nodosa, 14

Torreyochloa, 79 pallida var. fernaldii, 79 pallida var. pallida, 79

*Tovara virginiana, 81

Toxicodendron, 12, 98 pubescens, 12 radicans, 12

rydbergii, 12, 98

toxicarium, 12

toxicodendron, 12 vernix, 12

*Toxylon pomiferum, 61

"Tracaulon sagittatum, 81

*Trachelospermum difforme, 14

Tradescantia, 35, 103

"brevicaulis, 35

*incarnata, 35

ohiensis, 35 *ohiensis var. foliosa, 35

"reflexa, 35

"rosea, 35, 103

*rosea var. graminea, 35,103

virginiana, 35

*Tragiola pilosa, 68

Tragopogon, 26

dubius, 26

"major, 26

porrifolius, 26

pratensis, 26

Tragus, 79

berteronianus, 79

racemosus, 79

Trapa natans, 58

Trautvetteria caroliniensis, 84

*Triadenum, 52, 53, 106

fraseri, 52

tubulosum, 53, 106

virginicum, 53

walteri, 53

Triantha, 93, 115

glutinosa, 93, 115

racemosa, 93

Tribulus terrestris, 95

*Trichomanes intricatum, 7

Trichophorum planifolium, 43

Trichostema, 57

brachiatum, 57

dichotomum, 57

"dichotomum var. lineare, 57

*lineare, 57

setaceum, 57

*Triclisperma paucifolia, 80

*Tridax accedens, 19

Tridens, 71, 79

chapmanii, 79

flavus, 79

"flavus var. chapmanii, 79

"pulchellus, 71

*Trientalis borealis, 82

Trifolium, 49

"agrarium, 49

arvense, 49

aureum, 49

campestre, 49

dubium, 49

fragiferum, 49

bybridum, 49

incarnatum, 49

*incarnatum var. elatius, 49

medium, 49

pratense, 49

*procumbens, 49

reflexum, 49

"reflexum var. glabrum, 49

repens, 49

resupinatum, 49

virginicum, 49
Triglochin, 54, 106

maritima, 54, 106

striata, 54

Trigonella, 48, 49

caerulea, 49

foenum-graecum, 49

"monspeliaca, 48

"polycarpa, 48

Trillium, 60

cernuum, 60

*cernuum var. macranthum, 60

cuneatum, 60

erectum, 60

flexipes, 60

grandiflorum, 60

luteum, 60

nivale, 60

pusillum var. virginianum, 60

sessile, 60

undulatum, 60

*viride var. luteum, 60

Triodanis perfoliata, 31

Triosteum, 32

angustifolium, 32

aurantiacum, 32

perfoliatum, 32

Triphora trianthophora, 65

Tripidium ravennae, 79

Triplasis, 79

*intermedia, 79

purpurea, 79

Tripleurospermum, 26, 101

inodorum, 26, 101

maritimum ssp. maritimum, 26

"perforata, 26

"perforatum, 101

Tripsacum dactyloides, 79

Triticum aestivum, 79

Tsuga canadensis, 9

Tulipa sylvestris, 58

*Tunica prolifera, 33

Turritis glabra, 31

Tussilago farfara, 26

Typha, 93

angustifolia, 93

domingensis, 93

latifolia, 93

Ulmus, 93, 115

alata, 93

americana, 93

"floridana, 93

*fulva, 93

glabra, 93, 115

minor, 93, 115

parvifolia, 93

"procera, 93, 115

pumila, 93

rubra, 93

thomasii, 93, 115

*UMBELLIFERAE, 12, 98 
Uniola, 70, 79

"latifolia, 70

"laxa, 70

paniculata, 79

Urochloa, 79

distachya, 79

mutica, 79

panicoides, 79

plantaginea, 79

platyphylla, 79

ramosa, 79

reptans, 79

*subquadripara, 79

texana, 79

villosa, 79

Urtica, 93, 116

ballotifolia, 93

dioica, 93

*dioica ssp. gracilis, 93

gracilis, 93

urens, 93, 116

Utricularia, 57

"biflora, 57

cornuta, 57

*fibrosa, 57

geminiscapa, 57

gibba, 57

inflata, 57

intermedia, 57

juncea, 57

macrorhiza, 57

"pumila, 57

purpurea, 57

radiata, 57

resupinata, 57

striata, 57

subulata, 57

*vulgaris ssp. macrorbiza, 57

Uvularia, 35

grandiflora, 35

perfoliata, 35

puberula, 35

"pudica, 35

sessilifolia, 35

Vaccaria, 34

bispanica, 34

"vaccaria, 34

Vaccinium, 45

angustifolium, 45

"angustifolium var.

laevifolium, 45

*angustifolium var. nigrum, 45

"atrococcum, 45

caesariense, 45

corymbosum, 45

formosum, 45

fuscatum, 45

macrocarpon, 45

myrtilloides, 45

oxycoccos, 45 pallidum, 45

stamineum, 45

"stamineum var. interius, 45

*stamineum var. neglectum, 45

*vacillans, 45

Vachellia constricta, 49

Valeriana, 32, 102

officinalis, 32, 102

panciflora, 32

Valerianella, 32, 102

chenopodiifolia, 32, 102

*intermedia, 32

locusta, 32

"olitoria, 32

"patellaria, 32

radiata, 32

umbilicata, 32

"woodsiana, 32

Vallisneria, 52

americana, 52

*spiralis, 52

Veratrum, 60

bybridum, 60

"latifolium, 60

parviflorum, 60

virginicum, 60

viride var. viride, 60

Verbascum, 91

blattaria, 91

lychnitis, 91

phlomoides, 91

sinuatum, 91

thapsus, 91

Verbena, 93, 116

"angustifolia, 93

*bipinnatifida, 93

bonariensis, 93

bracteata, 93

"bracteosa, 93

brasiliensis, 93

hastata, 93

neomexicana, 93, 116

officinalis, 93

scabra, 93

simplex, 93

urticifolia, 93

Verbesina, 26, 101

alternifolia, 26

encelioides, 26

"encelioides var. exauriculata, 26

occidentalis, 26

virginica, 26, 101

Vernonia, 26

*altissima, 26

arkansana, 26

gigantea, 26

"gigantea ssp. ovalifolia, 26

glauca, 26

"harperi, 26

"maxima, 26

noveboracensis, 26

*ovalifolia, 26
Veronica, 68, 109

agrestis, 68

americana, 68

anagallis-aquatica, 68

arvensis, 68

austriaca ssp. teucrium, 68, 109

beccabunga, 68, 109

chamaedrys, 68

cymbalaria, 68, 109

filiformis, 68

*glandifera, 68

hederifolia, 68

"latifolia, 68, 109

longifolia, 68

officinalis, 68

peregrina ssp. peregrina, 68

persica, 68

polita, 68

scutellata, 68

serpyllifolia var.

serpyllifolia, 68

sublobata, 68

Veronicastrum virginicum, 68

Viburnum, 9, 97

acerifolium, 9

*alnifolium, 9

cassinoides, 9

*densiflorum, 9

dentatum var. dentatum, 9

dentatum var. lucidum, 9

dilatatum, 9

lantana, 9

lantanoides, 9

lentago, 9

molle, 9, 97

nudum, 9

opulus var. opulus, 9

prunifolium, 9, 97

rafinesquianum, 9

*recognitum, 9

rufidulum, 9, 97

"semitomentosum, 9

setigerum, 9

sieboldii, 9

Vicia, 49-50, 105

americana var. americana, 49, 105

*asycarpa, 50

*ativa nigra, 50

caroliniana, 50

cracca, 50

faba, 50

grandiflora, 50

hirsuta, 50

lathyroides, 50

narbonensis, 50

"ngustifolia, 50

peregrina, 50

sativa ssp. sativa, 50

tetrasperma, 50

"ugeri, 50

villosa ssp. varia, 50

villosa ssp. villosa, 50
Vigna, 50

"atjang, 50

*inensis, 50

mungo, 50

unguiculata, 50

Vinca, 14

major, 14

minor, 14

Vincetoxicum, 14

"carolinense, 14

"hirsutum, 14

nigrum, 14

Viola, 94, 116

affinis, 94

appalachiensis, 94

arvensis, 94

bicolor, 94

blanda, 94

*blanda var. palustriformis, 94

brittoniana, 94

canadensis, 94

*onspersa, 94

cucullata, 94

"emarginata, 94

*eriocarpa var. eriocarpa, 94

*eriocarpa var. leiocarpa, 94

esculenta, 94

*fimbriatula, 94

*foridana, 94

hastata, 94

hirsutula, 94

*incognita, 94

"kitaibeliana, 94

labradorica, 94

lanceolata, 94

*lanceolata ssp. vittata, 94

macloskeyi ssp. pallens, 94

odorata, 94

"pallens, 94

palmata, 94

*palmata var. sororia, 94

"papilionacea, 94

patrinii, 94, 116

pedata, 94

"pensylvanica, 94

"priceana, 94

primulifolia, 94

"pubescens var. eriocarpa, 94

"pubescens var. leiocarpa, 94

pubescens var pubescens, 94

pubescens var. scabriuscula, 94

"rafinesquei, 94

*rosacea, 94

rostrata, 94

rotundifolia, 94

sagittata, 94

*sagittata var. ovata, 94

*scabriuscula, 94 
septemloba, 94, 116

septentrionalis, 94, 116

sororia, 94

*sororia var.

missouriensis, 94

stoneana, 94

striata, 94

*subsinuata, 94

tricolor, 94

"triloba, 94

*triloba var. dilatata, 94

tripartita, 94, 116

*tripartita var. glaberrima, 94, 116

*vittata, 94

"walteri var. appalachiensis, 94

Vitex, 57, 107 agnus-castus, 57, 107

negundo, 57, 107

"negundo var. heterophylla, 57, 107 rotundifolia, 57

Vitis, 94

aestivalis, 94

"aestivalis var. bicolor, 94

cinerea var. floridana, 94

labrusca, 94

riparia, 94

rotundifolia var.

rotundifolia, 95

rupestris, 95

*simpsonii, 94

vulpina, 95
Vittaria appalachiana, 8

"Vulpia, 74

elliotea, 74

myuros, 74

octoflora, 74

octoflora var. octoflora, 74

sciurea, 74

"Waldsteinia fragarioides ssp. fragarioides, 86

"Washingtonia, 13 claytonii, 13 longistylis, 13

"Willoughbya scandens, 22

Wisteria, 50

floribunda, 50

frutescens, 50

sinensis, 50

Wolffia, 15, 99 borealis, 15, 99

brasiliensis, 15

columbiana, 15

"floridana, 15

"papulifera, 15

*punctata, 15, 99

Wolffiella gladiata, 15

Woodsia, 8

ilvensis, 8

obtusa ssp. obtusa, 8

"Woodwardia, 6

areolata, 6

virginica, 6
Xanthium, 26

"americanum, 26

"canadense, 26

"chinense, 26

"cylindraceum, 26

*echinatum, 26

*echinellum, 26

*pensylvanicum, 26

"speciosum, 26

spinosum, 26

strumarium, 26

"strumarium var. canadense, 26

"strumarium var. glabratum, 26

Xanthorhiza, 84

"apiifolia, 84

simplicissima, 84

*Xanthoxylum

americanum, 89

Xerophyllum asphodeloides, 60, 108

*Ximenesia encelioides, 26

*Xylosteon fragrantissimum, 32

Xyris, 95, 116

caroliniana, 95, 116

difformis var. difformis, 95

fimbriata, 95

jupicai, 95

smalliana, 95

torta, 95
Youngia, 26

japonica, 26

thunbergiana, 26

Yucca, 16

filamentosa, 16

flaccida, 16

*smalliana, 16

*Yulania acuminata, 59

Zannichellia palustris, 82

Zanthoxylum americanum, 89

Zea, 79, 111

mays, 79

mexicana, 79, 111

Zephyranthes atamasca, 12

*Zigadenus leimanthoides, 60

Zizania, 79, 111

*aquatica var.

angustifolia, 79, 111

aquatica var. aquatica, 79

palustris var. palustris, 79, 111

Zizaniopsis miliacea, 79

Zizia, 14

aptera, 14

*arenicola, 14

aurea, 14

"bebbii, 14

"cordata, 14

trifoliata, 14

Zostera marina var. stenophylla, 95

*Zosterella dubia, 82

Zoysia japonica, 79 



\section{SUMMARY OF REQUIREMENTS FOR SMITHSONIAN CONTRIBUTIONS SERIES}

For comprehensive guidelines and specifications, visit https://scholarlypress.si.edu.

ABSTRACTS must not exceed 300 words.

TEXT must be prepared in a recent version of Microsoft Word; use a Times font in 12 point for regular text; be doublespaced; and have 1" margins.

REQUIRED ELEMENTS are title page, abstract, table of contents, main text, and references.

FIGURES must be numbered sequentially (1, 2, 3, etc.) in the order called out; have components lettered consistently (in size, font, and style) and described in captions; include a scale bar or scale description, if appropriate; include any legends in or on figures rather than in captions. Figures must be original and must be submitted as individual TIF or EPS files.

FIGURE FILES must meet all required specifications in the Digital Art Preparation Guide. Color images should be requested only if required.

TAXONOMIC KEYS in natural history manuscripts should use the aligned-couplet form for zoology. If cross-referencing is required between key and text, do not include page references within the key, but number the keyed-out taxa, using the same numbers with their corresponding heads in the text.

SYNONYMY IN ZOOLOGY must use the short form (taxon, author, year:page), with full reference at the end of the manuscript under "References."

REFERENCES should be in alphabetical order, and in chronological order for same-author entries. Each reference should be cited at least once in main text. Complete bibliographic information must be included in all citations. Examples of the most common types of citations can be found at SISP's website under Resources/Guidelines \& Forms. 